\title{
Measurement of CP Asymmetries and Branching Fractions in Neutral B Meson Decays to Charged Pions and Kaons with the BABAR Detector *
}

\author{
Amir Farbin \\ Stanford Linear Accelerator Center \\ Stanford University \\ Stanford, CA 94309
}

SLAC-Report-740

Prepared for the Department of Energy under contract number DE-AC02-76SF00515

Printed in the United States of America. Available from the National Technical Information Service, U.S. Department of Commerce, 5285 Port Royal Road, Springfield, VA 22161.

*Ph.D. thesis, University of Maryland, College Park, MD 20742 


\begin{abstract}
Title of Dissertation: $\quad$ MEASUREMENT OF CP ASYMMETRIES

AND BRANCHING FRACTIONS IN

NEUTRAL B MESON DECAYS TO

CHARGED PIONS AND KAONS

WITH THE BABAR DETECTOR
\end{abstract}

Amir Farbin, Doctor of Philosophy, 2003

Dissertation directed by: Professor Abolhassan Jawahery

Department of Physics

This disseration presents a measurement of $\mathrm{CP}$ asymmetries and branching fractions for neutral $B$ meson decays to two-body final states of charged pions and kaons. The results are obtained from a data sample of about 88 million $\Upsilon(4 S) \rightarrow B \bar{B}$ decays collected between 1999 and 2002 with the BABAR detector at the PEP-II asymmetric-energy $B$ factory located at the Stanford Linear Accelerator Center. A fit to kinematic, topological, and particle identification information measures the charge-averaged branching fractions $\mathcal{B}\left(B^{0} \rightarrow \pi^{+} \pi^{-}\right)=(4.7 \pm 0.6 \pm 0.2) \times 10^{-6}$ and $\mathcal{B}\left(B^{0} \rightarrow K^{+} \pi^{-}\right)=(17.9 \pm 0.9 \pm 0.7) \times 10^{-6}$; the $90 \%$ confidence level upper limit $\mathcal{B}\left(B^{0} \rightarrow K^{+} K^{-}\right)<0.6 \times 10^{-6}$; and the direct CP-violating charge asymmetry $\mathcal{A}_{K \pi}=-0.102 \pm 0.050 \pm 0.016[-0.188,-0.016]$, where the first uncertainties are statistical and the second are systematic and the ranges in square brackets indicate the $90 \%$ confidence interval. A fit which adds decay time and $b$-flavor tagging information measures the CP-violating parameters for $B^{0} \rightarrow \pi^{+} \pi^{-}$decays $S_{\pi \pi}=0.02 \pm 0.34 \pm 0.05[-0.54,+0.58]$ and $C_{\pi \pi}=-0.30 \pm 0.25 \pm 0.04[-0.72,+0.12]$. 


\title{
MEASUREMENT OF CP ASYMMETRIES AND BRANCHING FRACTIONS IN NEUTRAL B MESON DECAYS TO CHARGED PIONS AND KAONS WITH THE BABAR DETECTOR
}

\author{
by \\ Amir Farbin \\ Dissertation submitted to the Faculty of the Graduate School of the \\ University of Maryland at College Park in partial fulfillment \\ of the requirements for the degree of \\ Doctor of Philosophy \\ 2003
}

Advisory Committee:

Professor Abolhassan Jawahery, Chairman/Advisor

Professor Rabindra N. Mohapatra

Associate Professor Stacy S. McGaugh

Associate Professor Sarah C. Eno

Assistant Professor Douglas Roberts 
(c) Copyright by

Amir Farbin

2003 


\section{Dedication}

The sacrifices of my parents provided to me the privalage of pursuing my dreams. This disseration is dedicated to my mother and father. 


\section{Acknowledgements}

Having been fortunate enough to be at the right place, during the right time, while working with right people, I realize that success is often the product of circumstance. I am very grateful to Hassan Jawahery for being the principle architect of this environment. I will remember his persuasive wisdom and inspiring teaching, which he kindly offered to me from our very first encounter. I also learned a great deal from Carlo Dallapiccola and Jim Olsen, who took upon themselves "to show me the ropes". Carlo's patient instruction and kind guidance was a rare treasure, and I had a lot of fun working with Jim, who thought me the virtues of attention to detail and diligence. I am also indebted to the members of the "two-body gang", all of whom were vital to the analysis presented in this dissertation. In particular, I thank Gianluca Cavoto who always did whatever was necessary to get the job done.

It was also my great fortune to have shared my BABAR experience with good friends. Shahram was always there to help with any problem, in- 
cluding writing a thesis. David and Sylvie always knew how to get me to relax. And Haleh took care of me at each step, sharing my every up and down.

I find myself surrounded by family and friends who give me strength and inspiration. Their support is my foundation. 


\section{Table of Contents}

List of Tables $\quad$ vi

List of Figures vii

1 Introduction 1

1.1 Matter/anti-matter and the Universe . . . . . . . . . . . . . 1

1.2 Overview of the contents . . . . . . . . . . . . . 3

2 Theory 5

2.1 A Brief History of Discrete Symmetries . . . . . . . . . . . . 5

2.2 The Standard Model of Fundamental Particles and Interactions . . . 8

2.2.1 The Building Blocks ................. . 9

2.2.2 CP Violation in the Standard Model . . . . . . . . . . . . . . 13

2.2.3 The CKM Matrix and Unitarity . . . . . . . . . . 14

2.2.4 The Unitarity Triangle . . . . . . . . . . . . . . 16

2.3 CP Violation Phenomenology . . . . . . . . . . . . . . . . . . 17

2.3.1 Strong and Weak Phases . . . . . . . . . . . . . . 18

2.3.2 Neutral Mesons . . . . . . . . . . . . . . . . . . . . . 19

2.3.3 CP Violating Observables .............. 21 
2.4 Neutral B Mesons . . . . . . . . . . . . . . . . . . . . . 22

2.4.1 Time Evolution of B's . . . . . . . . . . . . . . 23

2.4.2 Relating $\Lambda_{f}$ to the CKM matrix . . . . . . . . . . . 26

$2.5 \quad B^{0} \rightarrow \pi^{+} \pi^{-}, K^{+} \pi^{-} \ldots \ldots \ldots \ldots \ldots \ldots$

2.5.1 Disentangling Penguins and Trees using Isospin . . . . . . . . 30

2.5.2 SU(3) Flavor Symmetry . . . . . . . . . . . . . . . 32

2.5.3 Calculations of Hadronic Decay Amplitudes . . . . . . . . . . 33

3 The Detector $\quad 34$

3.1 PEP-II . . . . . . . . . . . . . . . . . . 35

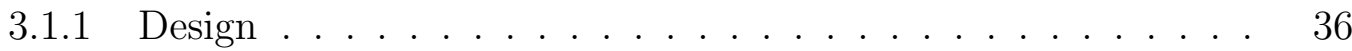

3.1.2 Operation and Performance . . . . . . . . . . . 36

3.2 BABAR . . . . . . . . . . . . . . . . . . . . . 38

3.2.1 Design considerations from PEP-II . . . . . . . . . . . . 41

3.2.2 Physics Constraints .................. 43

3.2.3 Tracking ...................... . . . 44

3.2.4 Charged Particle Identification . . . . . . . . . . . . . . . 47

3.2.5 Calorimetry ....................... 49

3.2.6 Muon Identification . . . . . . . . . . . . . . . . . 50

3.2.7 The Online System . . . . . . . . . . . . . . . 51

4 Analysis Computing $\quad 55$

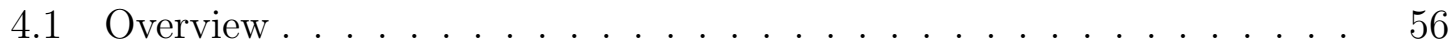

4.2 Event Reconstruction . . . . . . . . . . . . . . . . . . 58

4.2.1 Track Reconstruction . . . . . . . . . . . . . . 59

4.2.2 Particle Identification . . . . . . . . . . . . . . 64 
4.2 .3 Calorimetry . . . . . . . . . . . . . . . 66

4.2.4 Muon Identification . . . . . . . . . . . . . . . . . . . 68

4.3 Analysis Software . . . . . . . . . . . . . . . . . . . 69

4.3.1 Variables....................... . . 71

4.3.2 Measurement of Number of $B \bar{B}$ Events . . . . . . . . . . . . . 75

$\begin{array}{lll}5 & \text { Analysis Overview } & 78\end{array}$

5.1 The Task at Hand . . . . . . . . . . . . . . . . . . . . . 79

5.1.1 Kinematics ................... 80

5.1.2 Backgrounds.................... 81

5.1.3 Separation of Signal Modes . . . . . . . . . . . . . . . . 84

5.2 Extracting the Measurements . . . . . . . . . . . . . 89

5.2.1 Maximum Likelihood Fitting . . . . . . . . . . . . . . 91

5.2.2 The LMinuit Fitting Package . . . . . . . . . . . . . . . 94

5.2.3 The Toy Monte Carlo . . . . . . . . . . . . . . . . . . . 94

5.3 Analysis Outline . . . . . . . . . . . . . . . . . . 95

5.3.1 Data Sets ....................... 96

5.3.2 Selection Criteria and Efficiency . . . . . . . . . . . . 98

6 Measurement of the Branching Fractions 106

6.1 Maximum Likelihood fit for extracting Branching Fractions . . . . . . 107

6.1 .1 Fit Inputs . . . . . . . . . . . . . . . . . . . 108

6.1.2 Correlations Between Variables . . . . . . . . . . . . 118

6.1.3 Fit Parameters . . . . . . . . . . . . . . . . 118

6.2 Fit Validations . . . . . . . . . . . . . . . . . . 120

6.2.1 Toy Monte Carlo Tests . . . . . . . . . . . . . . . . 120 
6.2.2 Geant4 Monte Carlo Tests . . . . . . . . . . . . . . . . . 123

6.2 .3 Test Fits on Data . . . . . . . . . . . . . . . . . . . . . 124

6.3 Results . . . . . . . . . . . . . . . . . 126

6.3.1 Comparison with Toy Monte Carlos and Investigation of Possible Biases . . . . . . . . . . . . . . . . . . . . . . 128

6.3 .2 Plots . . . . . . . . . . . . . . . . . . . 131

6.3.3 Consistency of Fitted Signal Parameters . . . . . . . . . . . . 132

6.4 Systematics . . . . . . . . . . . . . . . . . . . 132

6.5 Summary . . . . . . . . . . . . . . . . 136

7 Ingredients of a Time-dependent CP Analysis 139

$7.1 \quad b$-Flavor Tagging . . . . . . . . . . . . . . . . 140

7.1.1 Flavor Tagging Decays . . . . . . . . . . . . . . 141

7.1.2 Combining Tag Signatures . . . . . . . . . . . . . . 145

7.1.3 Tagging Imperfections . . . . . . . . . . . . . . . 146

7.2 Measurement of $\Delta t \ldots \ldots \ldots \ldots \ldots$

7.2.1 The Algorithm . . . . . . . . . . . . . . . . . . . 149

7.3 The $\Delta t$ Resolution Function . . . . . . . . . . . . . . . . . . . . 151

7.4 The Self-tagged $B_{\text {Rec }}$ Sample . . . . . . . . . . . . . . . . 153

7.4.1 Selection .................... 153

7.4.2 The Composition . . . . . . . . . . . . . . 157

7.4 .3 ML Fit. . . . . . . . . . . . . . . . . 160

8 Analysis of the Time-dependent Asymmetries 167

8.1 Time Structure of Tagged $B^{0} \rightarrow h^{+} h^{-}$Decays . . . . . . . . . . . 169

8.2 Time and Flavor Structure of Background Candidates to $B^{0} \rightarrow h^{+} h^{\prime-} \quad 171$ 


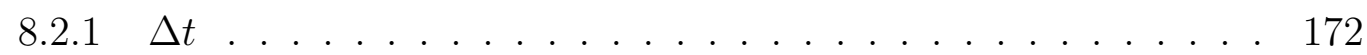

$8.2 .2 \quad$ Flavor Tagging . . . . . . . . . . . . . . . . 176

8.3 Correlation Studies . . . . . . . . . . . . . . . . . . . . . . 179

$8.3 .1 \quad \mathcal{F}$ and $\sigma_{\Delta t} \ldots \ldots \ldots \ldots \ldots \ldots \ldots \ldots$

8.3.2 $\mathcal{F}$ and Tagging Category . . . . . . . . . . . . 181

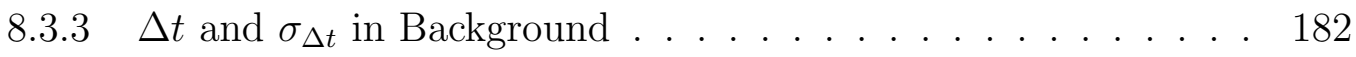

8.3.4 $\sigma_{\Delta t}$ and Tagging Category $\ldots \ldots \ldots \ldots \ldots$

8.3.5 Background $m_{\mathrm{ES}}$ and Tagging Category . . . . . . . . . . 184

8.4 Maximum Likelihood Fit . . . . . . . . . . . . . . . . . . . . . 184

8.5 Validations $\ldots \ldots \ldots \ldots \ldots \ldots \ldots \ldots \ldots$

8.5.1 Toy Monte Carlo . . . . . . . . . . . . . . . . . . . . 186

8.5.2 Geant4 Monte Carlo . . . . . . . . . . . . . . . . . . . 193

$8.5 .3 \quad B^{0} \rightarrow K^{+} \pi^{-}$Decays . . . . . . . . . . . . . . 194

8.6 Results . . . . . . . . . . . . . . . . . . . . . . 196

8.6.1 Alternative Measurements of $C_{\pi \pi} \ldots \ldots \ldots \ldots$

8.6 .2 Fit without $\mathcal{F} \ldots \ldots \ldots \ldots \ldots \ldots$

8.6 .3 Yields . . . . . . . . . . . . . . . . . . . . . . 198

8.6.4 Goodness of Fit . . . . . . . . . . . . . . . . . . . . 199

8.7 Systematics . . . . . . . . . . . . . . . . . . . . 199

9 Conclusions 204

9.1 The CKM matrix without $B^{0} \rightarrow h^{+} h^{-}$decays $\ldots \ldots \ldots \ldots$

9.2 Interpreting $S_{\pi \pi}$ and $C_{\pi \pi} \ldots \ldots \ldots \ldots \ldots \ldots \ldots$

9.3 Constraints from $B^{0} \rightarrow K^{+} \pi^{-} \ldots \ldots \ldots \ldots \ldots \ldots$

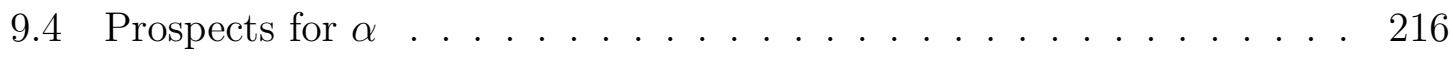


A Uncertainties on Time-dependent Measurements

B Comment on Information on $C_{\pi \pi}$ from $\Delta t$ Shape 


\section{List of Tables}

2.1 The fundamental particles and their quantum numbers. . . . . . . . 7

2.2 The fundamental interactions and their mediating particles. . . . . . 7

2.3 The Minimal Standard Model matter fields. The $L$ and $R$ subscripts indicate left and right-handed fields, respectively. The $i=1,2,3$ index enumerates the generations. The $\alpha=r, g, b$ is used for the $S U(3)$ transformations of the quarks. The $U(1)$ column lists the hyper-charge, while $S U(2)$ and $S U(3)$ columns list the dimension of representation of the fields under the respective gauge transformation. . . . . . . . . 10

3.1 Approximate production cross sections at PEP-II, including the experimental acceptance of BABAR. . . . . . . . . . . . . . . 37

3.2 PEP-II design and highest luminosity operating parameters. . . . . . 37 
3.3 Overview of the coverage, segmentation, and performance of the BABAR detector systems. The notation (C), (F), and (B) refers to the central barrel, forward and backward components of the system, respectively. The detector coverage in the laboratory frame is specified in terms of the polar angles $\theta_{1}$ (forward) and $\theta_{2}$ (backward). The number of readout channels is listed. Performance numbers are quoted for $1 \mathrm{GeV} / c$ particles, except where noted. The performances for the SVT and DCH are quoted for a combined Kalman fit (for the definition of the track parameters, see Section 4.2.1.) . . . . . . . . . . . . . . . . . . 42

3.4 Properties of $\mathrm{CsI}(\mathrm{Tl}) \ldots \ldots \ldots \ldots$

3.5 Cross sections, production and trigger rates for the principal physics processes at $10.58 \mathrm{GeV}$ for a luminosity of $3 \times 10^{33} \mathrm{~cm}^{-2} \mathrm{~s}^{-1}$. The $e^{+} e^{-}$ cross section refers to events with either the $e^{+}, e^{-}$, or both inside the EMC detection volume. . . . . . . . . . . . . . . .

5.1 Summary of detection efficiencies for $\pi^{+} \pi^{-}, K^{+} \pi^{-}$, and $K^{+} K^{-}$as determined in Geant4 signal Monte Carlo samples with about 40k events. The tracking correction accounts for measured tracking efficiency differences between simulated and recorded data. The efficiency of each cut is relative to the previous one and the errors are statistical, except for the PID and tracking efficiency corrections (which are included in the error on the total efficiency). For a description of each cut, refer

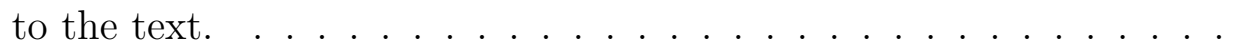


5.2 Selection efficiencies for non-hadronic $e^{+} e^{-} \rightarrow \tau \bar{\tau}$ and $e^{+} e^{-} \rightarrow e^{+} e^{-}$ events from Geant4 Monte Carlo. The reconstruction efficiency is relative to the number of generated events in each Monte Carlo sample, and the efficiency of each successive cut is relative to the previous one. The "expected yield" is the estimated number of events of the specific background source in the $B^{0} \rightarrow h^{+} h^{-}$sample. In order to estimate the background contributions to each signal decay, the $\pi \pi, K \pi$, and $K K$ "select" entries reflect the number of events in each Monte Carlo background sample which are consistent with the specific signal final state. These events are selected using particle identification and three standard deviation cuts on $m_{\mathrm{ES}}$ and $\Delta E$. The listed efficiency for these entries is relative to the $\Delta t, \sigma_{\Delta} t$ cut. $\ldots \ldots \ldots \ldots 2$

5.3 Selection efficiencies for $B$ decays. $B^{0} \rightarrow h^{+} h^{\prime-}$ events have been removed from these samples. The charmless sample is a cocktail of $B$ decays to final states without a $c$-quark. The caption of table 5.2 describes the contents of this table. . . . . . . . . . . . . 103

5.4 Selection efficiencies for charmless $B$ decays which may contribute to the $B^{0} \rightarrow h^{+} h^{\prime-}$ sample, from Geant4 Monte Carlo. Monte Carlo sample listed in the previous section which had no $B^{0} \rightarrow h^{+} h^{\prime-}$ candidates reconstructed are not tabulated.The caption of table 5.2 describes the contents of this table. . . . . . . . . . . . . . . . . . . 104

5.5 Selection efficiencies for continuum $e^{+} e^{-} \rightarrow u \bar{u}, d \bar{d}, s \bar{s}$, and $e^{+} e^{-} \rightarrow c \bar{c}$ events from Geant4 Monte Carlo. The caption of table 5.2 describes the contents of this table. . . . . . . . . . . . . 105 
6.1 Summary of PDFs and their parameters in the maximum likelihood fit. Parameters which are determined by the fit (ie floating) have no value or source specified. The indices' $k$ indicate species. . . . . . . . 108

6.2 Comparison of data and Monte Carlo $\Delta E$ resolution in $B^{-} \rightarrow D^{0} \pi^{-} \rightarrow$ $\left(K^{-} \pi^{+}\right) \pi^{-}$decays for different DCH high voltages. The fits to the data are displayed in figure 6.4. . . . . . . . . . . . . . . .

6.3 Comparison of data and Monte Carlo $\Delta E$ mean in $B^{-} \rightarrow D^{0} \pi^{-} \rightarrow$ $\left(K^{-} \pi^{+}\right) \pi^{-}$decays for difference DCH high voltages. The fits to the data are displayed in figure 6.4. . . . . . . . . . . . . . . . 113

6.4 Linear correlation coefficients for the variables $\left\{m_{\mathrm{ES}}, \Delta E, \mathcal{F}, \theta_{c}^{+}, \theta_{c}^{-}\right\}$ calculated for events in the $5.2<m_{\mathrm{ES}}<5.26 \mathrm{GeV} / c^{2}$ sideband region and the $B^{0} \rightarrow \pi^{+} \pi^{-}$Monte Carlo events. . . . . . . . . . . . . .

6.5 Summary of parameters in the branching fraction maximum likelihood

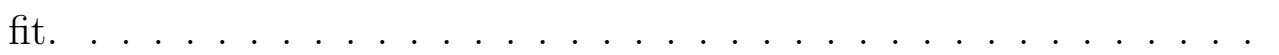

6.6 Summary of test fits on signal Monte Carlo samples of 50,000 $B^{0} \rightarrow$ $\pi^{+} \pi^{-}$and 200,000 $B^{0} \rightarrow K^{+} \pi^{-}$events. The choice of number of events roughly approximates the ratio of the branching fractions. . . . . . . 124

6.7 Summary of test fits on the continuum Monte Carlo samples, with and without appropriate number of signal events mixed in. . . . . . . . . 125

6.8 Summary of ML fit on the off-resonance data sample. . . . . . . . . . 126

6.9 The values of parameters from the branching fraction fit to the full on-resonance dataset. . . . . . . . . . . . . . . . . . . . 127

6.10 Comparison of the nominal values of signal PDF parameters and their fitted values on the data. The errors listed on the nominal values are used in the evaluation of the systematic error. . . . . . . . . . . 
6.11 Detailed summary of systematic errors on yields and CP asymmetries due to uncertainty in the parameterization of the PDFs. . . . . . . 137

6.12 Global summary of systematic errors on branching fractions and CP asymmetry. . . . . . . . . . . . . . . . . . 137

6.13 Summary of branching fraction results in the on-resonance $(87.9 \pm 1.0$ million $B \bar{B}$ pairs). The errors are statistical and systematic, respectively. 138

7.1 The measured branching fraction of the fully reconstructed self tagging $B$ decays in the $B_{\text {Rec }}$ sample. . . . . . . . . . . . . . . . . . . 154

7.2 Particles with $u, d$, and/or $s$ quarks used in the reconstruction of $B$ mesons. Composite particles rely on those above them. * designates a Breit-Wigner width. . . . . . . . . . . . . . . . . 154

7.3 Selected decay modes of $\bar{D}^{0}$ and $D^{-}$mesons and their branching fractions.

7.4 Selection criteria for $B^{0} \rightarrow D^{*-} \pi^{+} / \rho^{+} / a_{1}^{+}$decays. . . . . . . . . . 156

7.5 Selection criteria for $B^{0} \rightarrow D^{-} \pi^{+} / \rho^{+} / a_{1}^{+}$decays. . . . . . . . . . 157

7.6 Signal yield $N_{\text {sig }}$, purity $\mathcal{P}, \Delta E$ resolution $\sigma_{\Delta E}$, and $m_{\mathrm{ES}}$ resolution $\sigma_{m_{\mathrm{ES}}}$ for all reconstructed $B^{0}$ flavor eigenstates. . . . . . . . . . . 158

7.7 $\Delta t$ resolution function parameters extracted from the simultaneous fit to the $B_{\text {Rec }}$ and $B^{0} \rightarrow h^{+} h^{-}$samples. . . . . . . . . . . . . . . 162

7.8 Tagging parameters extracted from the simultaneous fit to the $B_{\text {Rec }}$ and $B^{0} \rightarrow h^{+} h^{\prime-}$ samples. See 7.1.1 for the definition of the categories. 163

7.9 Signal yield and $m_{\mathrm{ES}}$ parameters extracted from the simultaneous fit to the $B_{\text {Rec }}$ and $B^{0} \rightarrow h^{+} h^{-}$samples. . . . . . . . . . . . . . . . 164

7.10 Background $\Delta t$ parameters extracted from the simultaneous fit to the $B_{\text {Rec }}$ and $B^{0} \rightarrow h^{+} h^{-}$samples. . . . . . . . . . . . . . . 165 
7.11 Background tagging parameters extracted from the simultaneous fit to the $B_{\text {Rec }}$ and $B^{0} \rightarrow h^{+} h^{-}$samples. . . . . . . . . . . . . . . 165

7.12 Background yield and $m_{\mathrm{ES}}$ parameters extracted from the simultaneous fit to the $B_{\text {Rec }}$ and $B^{0} \rightarrow h^{+} h^{-}$samples. . . . . . . . . . . . 166

8.1 $\Delta t$ resolution function parameters extracted from simulated $B^{0} \rightarrow$ $D^{(*)-} \pi^{+} / \rho^{+} / a_{1}^{+}$and $B^{0} \rightarrow \pi^{+} \pi^{-}$events. . . . . . . . . . . . . . 172

8.2 Tagging parameters extracted from simulated $B^{0} \rightarrow D^{(*)-} \pi^{+} / \rho^{+} / a_{1}^{+}$ and $B^{0} \rightarrow K^{+} \pi^{-}$events. . . . . . . . . . . . . . . . . . 173

8.3 Linear correlation coefficients for the variables $\left\{m_{\mathrm{ES}}, \Delta E, \mathcal{F}, \theta_{c}^{+}, \theta_{c}^{-}, \Delta t, \sigma_{\Delta t}, \frac{\Delta t}{\sigma_{\Delta t}}\right\}$. The on-resonance sideband region is defined as $5.2<m_{\mathrm{ES}}<5.26 \mathrm{GeV} / c^{2} .180$

8.4 Summary of $B^{0} \rightarrow h^{+} h^{\prime-}$ yields in the CP maximum likelihood fit. . 186

8.5 Summary of $B^{0} \rightarrow h^{+} h^{\prime-}$ background $m_{\mathrm{ES}}, \Delta E$, and $\mathcal{F}$ parameters in the CP maximum likelihood fit. . . . . . . . . . . . . . . . . . 187

8.6 Summary of $B^{0} \rightarrow h^{+} h^{-}$background tagging efficiencies in the CP maximum likelihood fit. . . . . . . . . . . . . . . . . . . . 188

8.7 Summary of $B^{0} \rightarrow h^{+} h^{--}$background tagging asymmetries in the CP maximum likelihood fit. . . . . . . . . . . . . . . . . 189

8.8 Summary of $B^{0} \rightarrow h^{+} h^{-}$background $\Delta t$ parameters in the CP maximum likelihood fit. . . . . . . . . . . . . . . . . . . 190

8.9 Summary of test fits on signal Monte Carlo samples of 50,000 $B^{0} \rightarrow$ $\pi^{+} \pi^{-}$and 200,000 $B^{0} \rightarrow K^{+} \pi^{-}$events with $S_{\pi \pi}=-0.40, C_{\pi \pi}=0$, $\tau_{B}=1.54 \mathrm{ps}$, and $\Delta m_{d}=0.472 / \mathrm{ps}$. The choice of number of events roughly approximates the ratio of the branching fractions. . . . . . . 194

8.10 Detailed summary of systematic errors on yields and $C P$ asymmetries due to uncertainties in the PDF parameterizations, $\tau_{B}$, and $\Delta m_{d}$. . . 202 
8.11 Global summary of systematic errors on $C P$ asymmetries. . . . . . . . 202

8.12 Central values and $90 \%$ C.L. intervals for $S_{\pi \pi}$ and $C_{\pi \pi}$ from the maximum likelihood fit. The errors are statistical and systematic, respectively.203

9.1 Compilation of results on $B \rightarrow h h^{\prime}$ branching fractions (in units of $10^{-6}$ ) and direct CP asymmetries. Limits are quoted at 90\% CL. The world averages are computed taking into account asymmetric errors. 206

9.2 Inputs to the global CKM fit. If not stated otherwise: the first error includes statistics and experimental systematics and the second represent the systematic theoretical uncertainties. . . . . . . . . . 208 


\section{List of Figures}

2.1 The Unitarity Triangle. . . . . . . . . . . . . . . . . . 16

2.2 The Unitarity Triangle in the $\rho-\eta$ plane. . . . . . . . . . . . . . 17

2.3 The leading diagrams contributing to $B^{0}-\bar{B}^{0}$ mixing. . . . . . . . . 23

$2.4 B \rightarrow J / \psi K_{s}$ tree and penguin diagrams. . . . . . . . . . . . . . 26

$2.5 B \rightarrow u \bar{u} d$ and $B \rightarrow u \bar{u} s$ tree diagrams. . . . . . . . . . . . . 29

$2.6 \quad B \rightarrow u \bar{u} d$ and $B \rightarrow u \bar{u} s$ penguin diagrams. . . . . . . . . . . . . 29

2.7 The $B \rightarrow \pi \pi$ isospin triangles. . . . . . . . . . . . . . . . 31

3.1 The integrated PEP-II luminosities delivered to and recorded by BABAR. 38

$3.2 y-z$ cross-section schematic of the BABAR Detector. . . . . . . . . 39

3.3 The $x-y$ cross-section schematic of the BABAR Detector. . . . . . . . 40

$3.4 x-y$ cross-section schematic of the BABAR's SVT Detector. . . . . . 45

$3.5 y-z$ cross-section schematic of the BABAR's SVT Detector. . . . . . 45

3.6 Schematic of the 4 inner layers of the BABAR DCH. . . . . . . . . . . 46

3.7 Schematic drawing illustrating the detection of Cherenkov photons by BABAR's DIRC. . . . . . . . . . . . . . . . . . . . 48

$3.8 y-z$ Schematic drawing of the top half of BABAR's EMC. The detector is axially symmetric around the z-axis. All dimensions are in mm. $\quad$. 49

3.9 Schematic drawing the data path through BABAR's online system. . . 51

xviii 
4.1 A schematic diagram of the propagation of the recorded data from the detector to $\mathrm{HBOOK} / \mathrm{ROOT}$ where interactive analysis is performed. $\quad 58$

4.2 DCH position resolution as function of the drift distance in layer 18, for tracks on the left and right side of the sense wire, obtained from fits to track residual distributions in multi-hadron events. The data are averaged over all cells in the layer. . . . . . . . . . . . . . . . 60

4.3 SVT hit resolution in the (a) $z$ and (b) $\phi$ coordinate in microns, plotted as a function of track incident angle in degrees. . . . . . . . . . . . . 60

4.4 The differences (a) $\delta d_{0}$, (b) $\delta z_{0}$, (c) $\delta \phi_{0}$, and (d) $\delta \tan \lambda$ between fitted track parameters of the two halves of cosmic ray muons with $p_{t}>3 \mathrm{GeV}$. 61

4.5 The transverse momentum resolution determined from cosmic ray muons. 62

4.6 Data and Monte Carlo absolute tracking efficiency of good tracks in bins of track $p_{T}, \theta, \phi$, and number of tracks in the event, measured in runs with DCH high voltage of $1930 \mathrm{~V} . \ldots . . . . . . . . .$.

4.7 DCH $d E / d x$ measurements as a function of track momenta. The solid curves represent the Bethe-Bloch expectations for various mass hypotheses. . . . . . . . . . . . . . . . .

4.8 (a) The $\theta_{c}$ of kaons and pions versus the track momenta, and (b) the separation in standard deviations between pions and kaons as function of momentum, from the control sample described in section 5.1.3. . .

4.9 (a) The $\pi^{0}$ mass distribution reconstructed from two photon candidates in hadronic events overlaid with a fit to the data. (b) The ratio of measured to expected energy for electrons in Bhabha events overlaid with a Gaussian fit. The expected value is calculated from the production angle. The resolution is $1.9 \%$. . . . . . . . . . . . 
4.10 The ratio $E / p$ of deposited energy in the EMC over the measured momentum by the tracking system for electrons from $\gamma \gamma \rightarrow$ eeee events. 68

4.11 Muon efficiency (left scale) and pion misidentification probability (right scale) as a function of the track momentum. . . . . . . . . . . . . 70

4.12 Muon efficiency from January 2000 to July 2002 as a function of the run number. ...................... . . . 70

4.13 Distributions of (a) the number of tracks and (b) the visible energy $\mathcal{W}$, in the main physics processes at the $\Upsilon(4 S)$ energy. The distributions are from simulated events and are normalized to the same area. . . . 73

4.14 (a) The $\left|\cos \theta_{s}\right|$ and (b) sphericity distributions for $B \bar{B}$ events with a $B^{0} \rightarrow \pi^{+} \pi^{-}$decay (dashed red) and continuum background (solid black) events. The distributions are from simulated events and are normalized to unit area. . . . . . . . . . . . . . .

4.15 The $R_{2}$ distribution for signal $B^{0} \rightarrow \pi^{+} \pi^{-}$(dashed red) and continuum background (solid black) events. The distributions are from simulated events and normalized to unit area. . . . . . . . . . . . . 76

5.1 The polar angle $(\theta)$ of one track in $B^{0} \rightarrow h^{+} h^{\prime-}$ decays versus the polar angle of the (a) other track and (b) the track momentum. . . . . .

5.2 The Fisher Discriminant distribution for signal Monte Carlo (left) and sideband background (right). The fits to these are a bifurcated Gaussian for signal and double-Gaussian for background. . . . . . . . . . . 84

5.3 The (a) $\Delta M=M\left(D^{*}\right)-M\left(D^{0}\right)$ mass difference and (b) $D^{0}$ mass in the control sample used for studying DIRC $\theta_{c}$ measurements. . . . . 
5.4 The measured $\theta_{c}$ resolution $\left(\sigma_{\pi, K}^{ \pm}(\cos \theta)\right)$ and bias $\left(\mu_{\pi, K}^{ \pm}(\cos \theta)\right)$ for positively (circles) and negatively charged (triangles) pions (left) and kaons

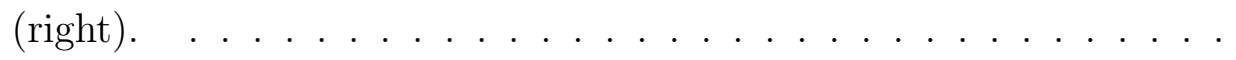

5.5 The mean and sigma of corrected $\theta_{c}$ pull distributions for positively (circles) and negatively (triangles) charged pions (left) and kaons (right) in bins of track momentum. The fits are to double Gaussian functions. 88

5.6 The corrected $\theta_{c}$ pull distributions for positively (left) and negatively (right) charged pions (top) and kaons (bottom). The fits are to double Gaussian functions. . . . . . . . . . . . . . . . . .

5.7 The $m_{\mathrm{ES}}$ and $\Delta E$ distributions of the 26,070 selected $B^{0} \rightarrow h^{+} h^{\prime-}$ candidates in the on-resonance sample.

6.1 Sample $m_{\mathrm{ES}}$ distributions in data for (a) signal $B^{-} \rightarrow D^{0} \pi^{-}$decays and (b) continuum $B^{0} \rightarrow h^{+} h^{-}$candidates in the sideband region $|\Delta E|>.15 \mathrm{GeV}$. The $B^{-} \rightarrow D^{0} \pi^{-}$events are fitted to a Gaussian plus an ARGUS function to account for continuum backgrounds. The continuum sample is fitted to an ARGUS function. . . . . . . . . . . 109

6.2 Distribution of $\Delta E$ for (a) signal $B^{0} \rightarrow \pi^{+} \pi^{-}$decays in Geant4 simulated events and (b) background continuum events from the sideband $m_{\mathrm{ES}}<5.26 \mathrm{GeV} / c^{2}$. The signal is fit with a Gaussian and the background is fit with a quadratic.

6.3 (a) Simulated $\Delta E$ distributions for (from right to left) $B^{0} \rightarrow \pi^{+} \pi^{-}$, $B^{0} \rightarrow K^{+} \pi^{-}$, and $B^{0} \rightarrow K^{+} K^{-}$decays reconstructed assuming both final state tracks are pions (a). (b) $\Delta E$ of simulated $B^{0} \rightarrow K^{+} \pi^{-}$ (green) and $B^{0} \rightarrow \pi^{+} \pi^{-}$(red) events versus the momentum of one of the final state tracks. . . . . . . . . . . . . . . . 
6.4 Distribution of $\Delta E$ for $B^{-} \rightarrow D^{0} \pi^{-} \rightarrow\left(K^{-} \pi^{+}\right) \pi^{-}$reconstructed events in data taken with DCH voltage at (a) $1960 \mathrm{~V}$, (b) $1900 \mathrm{~V}$, and (c) $1930 \mathrm{~V}$. The fits are to a Gaussian with a polynomial background component. . . . . . . . . . . . . . . . . . . 114

6.5 Distribution of $\mathcal{F}$ in signal $B^{0} \rightarrow \pi^{+} \pi^{-}$Monte Carlo (left) and onresonance $m_{\mathrm{ES}}<5.26 \mathrm{GeV} / c^{2}$ sideband data (right), on linear (top) and logarithmic (bottom) scales. The signal is fit to a bifurcated Gaussian and the background is to the sum of two Gaussians. . . . . . . . . 117

6.6 The distribution of the pulls of $N_{\pi \pi}^{S}, N_{K \pi}^{S}, \mathcal{A}_{K \pi}^{S}$, and $N_{K K}^{S}$ from fits to toy Monte Carlo experiments, fitted to Gaussian functions. . . . . . . 122

6.7 The distribution of fitted errors on $N_{\pi \pi}^{S}, N_{K \pi}^{S}$, and $\mathcal{A}_{K \pi}^{S}$, and the $-2 \log L=\chi^{2}-C$ of fits to 500 toy Monte Carlo experiments generated with the parameters obtained from the fit to the data. The errors indicate the result from the data fit. . . . . . . . . . . . .

6.8 The distribution of the difference between fitted $N_{\pi \pi}^{S}, N_{K \pi}^{S}, \mathcal{A}_{K \pi}^{S}$, and $N_{K K}^{S}$ and inputed values for 500 toy Monte Carlo experiments generated with the parameters obtained from the fit to the data. . . . . . . 130

6.9 Distributions of $m_{\mathrm{ES}}$ and $\Delta E$ for samples enhanced in (a,b) signal $\pi^{+} \pi^{-}$and (c,d) $K^{\mp} \pi^{ \pm}$decays. Solid curves represent projections of the maximum likelihood fit, dashed curves represent $q \bar{q}$ and $\pi \pi \leftrightarrow K \pi$ cross-feed background. . . . . . . . . . . . . . . . .

6.10 The Fisher discriminant distribution for a sample enhanced in signal decays. The solid curve represents the projection of the maximum likelihood fit. The dashed curve represents the signal contribution. . . 135 
7.1 Leading lepton producing neutral $B$ meson decays. The $b$-quark and lepton charges are correlated in (a) $B \rightarrow X l \nu$ (a) and anti-correlated in the $(\mathrm{b}) \bar{b} \rightarrow \bar{c} \rightarrow \bar{s} . \ldots \ldots \ldots 141$

7.2 An example of a $\bar{b} \rightarrow \bar{c} \rightarrow \bar{s}$ transition which produces kaons whose charge has both the same and opposite sign as the $b$-quark. . . . . . . 142

$7.3 B^{0} \rightarrow D^{*-} \pi^{+}, \rho^{+}, a_{1}^{+}$decays which produce a soft pion whose charge has the opposite sign as the $b$-quark. In contrast the $\pi^{+}, \rho^{+}$, or $a_{1}^{+}$ from the $B$ carries the same charge as the $b$-quark. . . . . . . . . . . 144

7.4 (a) the RMS spread and (b) the mean of the residual $\delta_{t}=\Delta t_{\text {meas }}-$ $\Delta t_{\text {true }}$ versus the measured $\sigma_{\Delta t}$ in simulated $B$ decays. . . . . . . . . 152

7.5 Distribution of the $m_{\mathrm{ES}}$ for selected $B^{0}$ candidates in flavor eigenstates. 159

8.1 The measured error on $B^{0} \rightarrow \pi^{+} \pi^{-}$and $B^{0} \rightarrow K^{+} \pi^{-}$yields from fits to toy Monte Carlo experiments, with (solid) and without (dashed) $\Delta t$ in the ML fit. . . . . . . . . . . . . . . . . . . . . . 168

8.2 The measured $\Delta t$ of $B^{0} \rightarrow h^{+} h^{--}$candidates from (in order of increasing width) simulated $e^{+} e^{-} \rightarrow u \bar{u}, d \bar{d}, s \bar{s}, e^{+} e^{-} \rightarrow c \bar{c}$, and signal $B^{0} \rightarrow$ $\pi^{+} \pi^{-}$events. The RMS of these distributions are $1.320 \pm 0.002 \mathrm{ps}$, $1.697 \pm 0.005 \mathrm{ps}$, and $2.370 \pm 0.007 \mathrm{ps}$, respectively. All distributions are normalized to same area. . . . . . . . . . . . . . . . 175

8.3 The $\Delta t$ distribution of candidate $B^{0}$ (solid) and $\bar{B}^{0}$ (dashed) $B^{0} \rightarrow$ $h^{+} h^{\prime-}$ decays (top), and their apparent CP asymmetry (bottom), for events in the sideband region $m_{\mathrm{ES}}<5.26 \mathrm{GeV} / c^{2}$. . . . . . . . . . 175 
8.4 The $\Delta t$ distribution of candidates in the $B^{0} \rightarrow h^{+} h^{\prime-}$ sample in the sideband region $m_{\mathrm{ES}}<5.26 \mathrm{GeV} / c^{2}$, fitted with $\mathcal{R}_{\mathrm{bkg}}\left(\Delta t ; \bar{v}_{\mathrm{EXP}}^{b k g}\right)$ (left) and $\mathcal{R}\left(\Delta t_{\text {true }}, \sigma_{\Delta t} ; \bar{v}_{3 G S}^{b k g}\right)$ (right). The top and middle plots display the data (points) and fit results (line), on linear and log scales, respectively. The bottom plot shows the residual of the data minus the fit function. 176

8.5 The $m_{\mathrm{ES}}$ distribution of the tagged events in the $B^{0} \rightarrow h^{+} h^{-}$sample, separated by tagging category. . . . . . . . . . . . . . . . 177

8.6 Mean (top) and RMS (bottom) of $\mathcal{F}$ versus the error on $\Delta t$. . . . . . 181

8.7 Fisher PDFs for signal (left) and $q \bar{q}$ (right) separated by tagging category. 182

8.8 Projection plots of the mean and RMS of $\Delta t$ as a function of $\sigma(\Delta t)$ from the on-resonance $m_{\mathrm{ES}}$ sideband sample. . . . . . . . . . . . . . . 183

8.9 Pull distributions of $S_{\pi \pi}$ and $C_{\pi \pi}$ from fits to toy Monte Carlo experiments. . . . . . . . . . . . . . . . . . 191

8.10 The mean and width of $S_{\pi \pi}$ and $C_{\pi \pi}$ as a function of their generated values from toy Monte Carlo experiments. . . . . . . . . . . . . . 192

8.11 The distributions of residual (measured minus generated values) of $S_{\pi \pi}$ and $C_{\pi \pi}$ from fits to toy Monte Carlo experiments. . . . . . . . . 193

8.12 The time-dependent mixing asymmetry, $A_{\mathrm{K} \pi}^{\operatorname{mix}}(\Delta t)$, from samples enriched in $B^{0} \rightarrow K^{+} \pi^{-}$and $\bar{B}^{0} \rightarrow K^{-} \pi^{+}$decays. The curve represents the expectation including all signal and background decays, calculated from the PDFs used in the fit. . . . . . . . . . . . . . . . . 195 
8.13 The $\Delta t$ distribution for (a) $B^{0}$ and (b) $\bar{B}^{0}$ tagged events and (c) the asymmetry $A_{\pi \pi}^{\mathrm{CP}}(\Delta t)$, from a sample enriched in $B^{0} \rightarrow \pi^{+} \pi^{-}$decays. The dashed curve represents the expected background contributions, primarily from continuum and $B^{0} \rightarrow K^{+} \pi^{-}$decays. The solid curve plots the signal and background contributions assuming the result from the fit to the full $B^{0} \rightarrow h^{+} h^{-}$sample. . . . . . . . . . . . . . . . 197

8.14 The error on $S_{\pi \pi}$ and $C_{\pi \pi}$ (left) and the $-2 \log$ likelihood from toy MC experiments (right). The arrows point to the values from the data fit. 200

9.1 Confidence levels in the $\bar{\rho}-\bar{\eta}$ place based on inputs listed in table 9.2. The shaded areas indicate regions of $\geq 90 \%$ and $\geq 5 \%$ CLs, respectively. Also shown are the $\geq 5 \% \mathrm{CL}$ domains of the individual constraints and the $\geq 32 \%$ and $\geq 5 \%$ CL constraints from the world

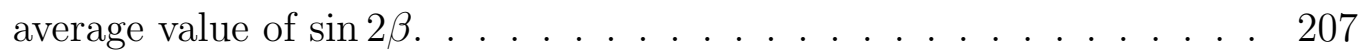

9.2 Confidence levels in the $\bar{\rho}-\bar{\eta}$ plane for strategy (1). Overlayed is the prediction from the SM fit. Refer to the text for a discussion. . . . . . 211

9.3 Confidence levels in the $\bar{\rho}-\bar{\eta}$ plane for strategy (2). Overlayed is the prediction from the SM fit. Refer to the text for a discussion. . . . . . 212

9.4 Confidence levels in the $\bar{\rho}-\bar{\eta}$ plane for strategy (3). Overlayed is the prediction from the SM fit. Refer to the text for a discussion. . . . . .

9.5 Confidence levels in the $\bar{\rho}-\bar{\eta}$ plane for strategy (4). Overlayed is the prediction from the SM fit. Refer to the text for a discussion. . . . . . 214

9.6 Confidence levels in $\alpha$ from strategies (1)-(4). The dashed-line/hashedarea represent the prediction from the SM fit. . . . . . . . . . . 215

9.7 The statistical errors on (a) $S_{\pi \pi}$ and (b) $C_{\pi \pi}$ versus total recorded luminosity at the $\Upsilon(4 S) . \ldots \ldots \ldots \ldots$. . . . . . . . . . . 217 
9.8 The residual $\theta=\alpha-\alpha_{\text {Eff }}$ at different recorded luminosities, using full isospin analysis with no electroweak penguins and including tagged branching fractions for $B^{0} \rightarrow \pi^{0} \pi^{0} \ldots \ldots \ldots \ldots \ldots \ldots$ 


\section{Chapter 1}

\section{Introduction}

\subsection{Matter/anti-matter and the Universe}

The scientific endeavor to unravel the mystery of existence is based on the premise that phenomena at the largest conceivable scale, ie what we observe as we look into space, is connected to the smallest known scale, i.e. what we know about subatomic particles, at the very beginning of the Universe. Perhaps the most convincing evidence to support this idea is that some 13 billion years after the instant we believe the Universe began, we observe the after-glow of the those early moments with the precise signature predicted by our theories. It would be specious, however, to imply the picture is complete and all is understood.

One seemingly striking inconsistency in this picture is that though our theory of fundamental sub-atomic particle interactions (the Standard Model) places matter and anti-matter at nearly equal footing, our Universe appears to be composed of only matter. Indeed the Standard Model accounts for small differences (or asymmetries) in the interactions of matter and anti-matter through a phenomena known as "CP violation", and we can conceive of how such a difference leads to the dominance of 
matter. Yet it falls short, by orders of magnitude, in accounting for the observed matter asymmetry in the Universe. Fortunately there are many reasons to believe that the Standard Model does not fully describe the fundamental laws leading to the modern Universe.

Though every experimental measurement of the properties of sub-atomic particles has precisely agreed with Standard Model predictions, a limitation of this model's construction is that it is not the fundamental theory, but is rather what is known as an "effective" theory describing phenomena to certain distance (or equivalently energy) scale. The physics of smaller (or higher energy) processes are obscured in parameters which must be measured, much like the measurement of a spring constant encapsulates the more complicated and detailed mechanics of a spring. What's more, we know that not only is the Standard Model incomplete, but that more importantly, there is physics beyond it which necessarily sheds light on unexplained phenomena.

Progress in physics is commonly the result of the observation of an inconsistency with an existing theory. Since the matter/anti-matter difference incorporated in the Standard Model is manifested in merely one parameter, it is an excellent candidate for revealing such an inconsistency. Furthermore our speculations (or rather our only viable predictions) of the physics beyond the Standard Model generally provide more sources of CP violation. Therefore investigations of the matter/anti-matter asymmetries hold great prospect for gathering hints of what lies beyond the Standard Model.

In the previous decade, two powerful particle accelerators were specifically built to study $\mathrm{CP}$ violation in the properties of a particle which promises to be an excellent probe of such phenomena: the $B$ meson. These colliders, known as asymmetric " $B$ factories", provide abundant samples of this particle in an environment condusive to 
detailed measurements of its behavior. At each factory a collaboration of physicists has assembled an array of detectors to precisely record the remnants of each $B$ meson's decay. A primary goal of these experiments is to look for inconsistencies in the Standard Model picture of CP violation. Typically this task is represented as a test of the closure of a triangle (i.e. its angles add to 180) whose angles $\alpha, \beta$, and $\gamma$ are related to decays of $B$ meson to different final states, and whose area reflects the magnitude of CP violation in the Standard Model. Already, these projects have measured $\sin 2 \beta$ to be $0.734 \pm 0.054$ [24], or more significantly, clearly not zero, and therefore established the $B$ meson as only the second particle which has been directly observed to demonstrate CP violating behavior in nearly 40 years of searching.

With the current precision, the Standard Model appears to correctly predict the value of $\beta$. In this decade, as records of $B$ meson decays accumulate, physicists will perform numerous new tests and refine their existing assessment of the consistency of the Standard Model's matter/anti-matter asymmetries. This dissertation focuses on the decays of neutral $B$ mesons to two particle final states containing charged kaons and pions. These decays promise to yield information on two other important angles, $\alpha$ and $\gamma$, which are vital ingredients in the $B$ factories' test of $\mathrm{CP}$ violation in the Standard Model. The analysis is based on data collected at the Stanford Linear Accelerator Center's asymmetric B factory, which is composed of the PEP-II electron/positron storage ring and the BABAR detector.

\subsection{Overview of the contents}

This dissertation attempts to portray a comprehensive picture of BABAR's analysis of the $B^{0}$ decays to the $\pi^{+} \pi^{-}, K^{+} \pi^{-}$, and $K^{+} K^{-}$final states. In order to motivate the 
study of these modes, the next chapter focuses on how the angles $\alpha, \beta$, and $\gamma$ relate the Standard Model, CP violation, and $B$ decays. Chapters 3 and 4 present an overview of the BABAR detector and its supporting software as a means of facilitating detailed discussions of the analysis in later chapters. Chapter 5 discusses the experimental challenges presented by the $B^{0} \rightarrow \pi^{+} \pi^{-}, K^{+} \pi^{-}, K^{+} K^{-}$decay modes and details the various techniques adopted for the analysis, eventually outlining its blueprint. Chapter 6 presents the extraction of the branching fractions for these modes. Chapter 7 surveys the remaining ingredients required for the full CP analysis of the $B^{0} \rightarrow \pi^{+} \pi^{-}$ decay, which is presented in chapter 8 . Finally chapter 9 evaluates the impact of the measurements presented in this dissertation on our knowledge of Standard Model parameters, and discusses future prospects. 


\section{Chapter 2}

\section{Theory}

Our understanding of the sub-atomic phenomena observed in high energy accelerators and detectors is encapsulated in the Standard Model (SM) of the electromagnetic, weak, and strong interactions. This chapter is a survey of how CP violation (CPV) in the SM is explored at the $B$ factories. We'll begin by summarizing the basic elements of SM, focusing on the CKM matrix in order to illuminate the origins of CPV in the theory. After studying the phenomology of CPV, we'll examine how the neutral $B$ decays relate to SM parameters. Finally, we'll focus on the specifics of two-body $B^{0}$ decays to charged pions and kaons, revealing the potential significance of the measurements described in this dissertation.

\subsection{A Brief History of Discrete Symmetries}

The discrete space-time operations of parity $(P: \mathbf{x} \rightarrow-\mathbf{x})$ and time-reversal $(T: t \rightarrow$ $-t$ ) have classical interpretations. Testing the parity conservation of a classical theory corresponds to validating the invariance of its laws of motion under a mirror reflection about a coordinate plane followed by $\pi$-rotation about the axes perpendicular to that plane. Similarly, time-reversal symmetry of a classical theory indicates no time 
direction preference. These operations were recognized long before the advent of quantum mechanics and quantum field theory as symmetries of classical theories of gravity and electromagnetism. Charge-conjugation (C) operation, however, was first brought to light by relativistic quantum theory's prediction of anti-particles. This operation, which corresponds to reversing all quantum numbers of a particle while keeping the mass unchanged, has no classical analogue.

Before the 1950's it was generally assumed that each of these discrete operations were fundamental symmetries of nature. In 1956 Lee and Yang [1], by surveying the available experimental evidence, determined that parity conservation was "only an extrapolated hypothesis." Soon afterwards Wu et al. [2] discovered parity violation in $\beta$ decay of ${ }^{60} \mathrm{Co}$, and Goldhaber et al. [3] found neutrinos emitted in electron capture by ${ }^{157} E u$ were all left-handed (i.e. had opposite spin and momentum directions), indicating $\mathrm{C}$ violation.

Charge-conjugation violation, however, was generally found in conjunction to $\mathrm{P}$ violation. As an illustration, consider charged pion decays to muons where out of the four possible transitions 1: $\pi^{+} \rightarrow \mu_{R}^{+} \nu_{\mu_{L}}, 2: \pi^{-} \rightarrow \mu_{L}^{-} \bar{\nu}_{\mu_{R}}, 3: \pi^{+} \rightarrow \mu_{L}^{+} \nu_{\mu_{R}}$, and 4: $\pi^{-} \rightarrow \mu_{R}^{-} \bar{\nu}_{\mu_{L}}$, only the first two have been observed. $\mathrm{P}$ violation accounts for the absence of the missing decays. However $\mathrm{C}$ is also violated because $C:(1) \rightarrow(4)$ and $C:(2) \rightarrow(3)$. Yet since the observed $\pi^{+}$and $\pi^{-}$decays are related by the combined $\mathrm{C}$ and $\mathrm{P}$ operations, $\mathrm{CP}$ is conserved.

CPV was first discovered by Christenson et al. [5] in 1964 with the discovery of the decay $K_{L} \rightarrow \pi \pi$. Soon afterwards Sakharov [4] suggested CPV to be one of the integral components of any mechanism leading to the matter/anti-matter asymmetry in our universe. In the next few decades, the SM, which encapsulates the KobayashiMaskawa [6] mechanism of CPV through flavor-changing charge currents between 


\begin{tabular}{ccccccccc}
\hline \hline & \multicolumn{3}{c}{ Family } & Electr. & \multicolumn{4}{c}{ Weak Charge } \\
Fermion & 1 & 2 & 3 & charge & Color & left-hd. & right-hd. & Spin \\
\hline Leptons & $\nu_{e}$ & $\nu_{\mu}$ & $\nu_{\tau}$ & 0 & $\mathrm{n} / \mathrm{a}$ & $\frac{1}{2}$ & $\mathrm{n} / \mathrm{a}$ & $\frac{1}{2}$ \\
& $e$ & $\mu$ & $\tau$ & -1 & $\mathrm{n} / \mathrm{a}$ & $-\frac{1}{2}$ & 0 & $\frac{1}{2}$ \\
\hline \multirow{2}{*}{ Quarks } & $u$ & $c$ & $t$ & $\frac{2}{3}$ & $\mathrm{r}, \mathrm{b}, \mathrm{g}$ & $\frac{1}{2}$ & 0 & $\frac{1}{2}$ \\
& $d$ & $s$ & $b$ & $-\frac{1}{3}$ & $\mathrm{r}, \mathrm{b}, \mathrm{g}$ & $-\frac{1}{2}$ & 0 & $\frac{1}{2}$ \\
\hline \hline
\end{tabular}

Table 2.1: The fundamental particles and their quantum numbers.

\begin{tabular}{cccc}
\hline \hline & Coupling & Particle(s) & Symmetry \\
Force & Charge & Exchanged & \\
\hline Electro-weak & Electric/weak & Photon $(\gamma), W^{ \pm}, Z^{0}$ & $U(1) \times S U(2)$ \\
Strong & Color & 8 Gluons $(g)$ & $S U(3)$ \\
\hline \hline
\end{tabular}

Table 2.2: The fundamental interactions and their mediating particles.

three generations of quarks, became established as the fundamental theory of particles and interactions.

There was no indication of CPV outside the kaon system until recently (2001), when the $B$ factories met their first major milestone and observed the phenomena in $B$ meson decays to $\mathrm{CP}$ eigenstates containing charmonium [7]. 


\subsection{The Standard Model of Fundamental Particles and Interactions}

The building blocks of the Standard Model are three fundamental realizations of particle physics:

1. three families of particles (listed in table 2.1), each consisting of two quarks and two leptons, are the building blocks for all matter, including the hundreds of exotic particles produced in high energy accelerators;

2. the interactions of these particles are the expression of three local gauge symmetries of nature (see table 2.2); and

3. interactions with a heavy scalar with a non-zero vacuum expectation value bestows mass on all of the particles and breaks the electo-weak gauge symmetry.

The aesthetic appeal of the SM is that the elegant interplay of these three basic elements leads to a theory that describes all known phenomena with high precision. In order to be predictive, the model relies on 18 experimentally inferable parameters, most of which are related to the mechanism by which all particles acquire mass: interactions with the still unobserved Higgs boson. In addition to particle masses, these Higgs-related parameters encapsulate the flavor changing processes (i.e. the mechanism by which one generation of quarks couples to another) in the CabibboKobayashi-Maskawa (CKM) matrix. The SM predicts that one parameter in this matrix is the only source of $\mathrm{CP}$ violating processes among quarks. 


\subsubsection{The Building Blocks}

Table 2.3 displays the Minimal Standard Model's (MSM) three generations of quarks and leptons and the spin-zero Higgs boson. Note that the suggestive notation used to express these fields are indicative of experimental observations. For example, the table lists no right-handed neutrino, reflecting that up to recently there was no evidence for such a particle.

The dynamics of each fermion is governed by a term $\bar{f} i \gamma^{\mu} \delta_{\mu} f$ in the Lagrangian, where $\gamma^{\mu}$ are Dirac matrices. The SM, however, requires that the theory obeys the gauge symmetries $U(1) \times S U(2) \times S U(3)$. This condition promotes the derivative in the fermion terms to a "co-variant" derivative

$$
\delta^{\mu} \rightarrow \mathcal{D}^{\mu}=\delta^{\mu}-i g_{1} \frac{Y}{2} B^{\mu}-i g_{2} \frac{\sigma_{i}}{2} W_{i}^{\mu}-i g_{3} \frac{\lambda_{a}}{2} G_{a}^{\mu}
$$

which introduces fermion interactions with new force mediating vector fields. Here the $g_{i}$ are the coupling constants; $Y, \sigma_{i}$, and $\lambda_{a}$ are the generators; and $B^{\mu}, W_{i}^{\mu}$, and $G_{a}^{\mu}$ are the mediators of the resulting interactions of the $U(1), S U(2)$, and $S U(3)$ symmetries, respectively.

Table 2.3 indicates how the fields transform under each of the gauge symmetries. Once again the table reflects experimental observations. The $S U(2)$ symmetry maximally violates parity, thus the left-handed fermion fields are doublets under $S U(2)$ transformations (and are therefore written in doublet form), while the right-handed fermions are $S U(2)$ singlets. Also leptons carry no strong charge and are represented by $S U(3)$ singlets with no color index $\alpha$.

The simplest model of the Higgs field with a vacuum expectation value adds

$$
\mathcal{L}_{\text {Higgs }}=\left(D_{\mu} H\right)^{\dagger}\left(D_{\mu} H\right)-\frac{\lambda}{4}\left(H^{\dagger} H-v^{2} / 2\right)^{2}
$$


Table 2.3: The Minimal Standard Model matter fields. The $L$ and $R$ subscripts indicate left and right-handed fields, respectively. The $i=1,2,3$ index enumerates the generations. The $\alpha=r, g, b$ is used for the $S U(3)$ transformations of the quarks. The $U(1)$ column lists the hyper-charge, while $S U(2)$ and $S U(3)$ columns list the dimension of representation of the fields under the respective gauge transformation.

\begin{tabular}{lccc}
\hline \hline Field & $U(1)$ & $S U(2)$ & $S U(3)$ \\
\hline$u_{R}^{i, \alpha}$ & $2 / 3$ & $\mathbf{1}$ & $\mathbf{3}$ \\
$d_{R}^{i, \alpha}$ & $-1 / 3$ & $\mathbf{1}$ & $\mathbf{3}$ \\
$e_{R}^{i}$ & -1 & $\mathbf{1}$ & $\mathbf{1}$ \\
$Q_{L}^{i}=\left(\begin{array}{c}u_{L}^{i, \alpha} \\
d_{L}^{i, \alpha}\end{array}\right)$ & $1 / 6$ & $\mathbf{2}$ & $\mathbf{3}$ \\
$L_{L}^{i}=\left(\begin{array}{c}\nu_{L}^{i} \\
e_{L}^{-}\end{array}\right)$ & $-1 / 2$ & $\mathbf{2}$ & $\mathbf{1}$ \\
$H=\left(\begin{array}{c}H^{+} \\
H^{0}\end{array}\right)$ & $1 / 2$ & $\mathbf{2}$ & $\mathbf{1}$ \\
\hline \hline
\end{tabular}


to the Lagrangian, where the last term is the Higgs potential which is symmetric under the gauge symmetry $Q: H \rightarrow e^{i \phi(x) Q} H$, where $Q=\frac{\sigma_{3}}{2}+\frac{Y}{2}$ is the electric charge operator. The $U(1) \times S U(2)$ electroweak gauge symmetry of the total Lagrangian is spontaneously broken when nature selects a particular configuration from the continuum of degenerate minima of the Higgs potential. Expanding about this specific minimum and using gauge transformations to eliminate any massless components of the Higgs,

$$
H(x)=\left(\begin{array}{c}
0 \\
v / \sqrt{2}+\operatorname{Re} h^{0}(x)
\end{array}\right) .
$$

As a consquence, the $W^{ \pm}$and $Z$ bosons acquire masses from the dynamic terms of the Higgs Lagrangian ${ }^{1}$ :

$$
\mathcal{L}=\frac{g_{2}^{2} v^{2}}{8}\left(W_{1} W_{1}+W_{2} W_{2}\right)+\frac{v^{2}}{8}\left(g_{2} W_{3}-g_{1} B\right)^{2}
$$

The massive charged bosons are then recognized from this expression as $W^{ \pm}=$ $\frac{W^{1} \mp i W^{2}}{\sqrt{2}}$, with mass $M_{W}=\frac{g_{2} v}{2}$. Similarity the neutral boson is $Z=\cos \theta_{W} W^{3}-$ $\sin \theta_{W} B$ with mass $M_{z}=\frac{M_{W}}{\cos \theta_{W}}$, and the massless photon is $A=\sin \theta_{W} W^{3}+\cos \theta_{W} B$, where $\tan \theta_{w} \equiv g_{1} / g_{2}$.

The introduction of Yukawa couplings of the Higgs to the fermions:

$$
\mathcal{L}_{\text {Yukawa }}=g_{u}^{i j} \bar{u}_{R}^{i} H^{T}\left(-\sigma_{1} \sigma_{2}\right) Q_{L}^{j}-g_{d}^{i j} \bar{d}_{R}^{i} H^{\dagger} Q_{L}^{j}-g_{e}^{i j} \bar{e}_{R}^{i} H^{\dagger} L_{L}^{j}+\text { h.c. },
$$

introduces fermion mass terms when the Higgs is expanded about its minimum, but still retains the gauge invariance of the Lagrangian. Here $\sigma_{i}$ are the Pauli matrices. The mass matrices

$$
\mathcal{M}_{u}=v g_{u} / \sqrt{2}, \mathcal{M}_{d}=v g_{d} / \sqrt{2}, \mathcal{M}_{e}=v g_{e} / \sqrt{2}
$$

\footnotetext{
${ }^{1}$ In order to ease notation, color and Lorentz indices are suppressed when possible.
} 
are not necessarily diagonal, so these Yukawa couplings also introduce mixing between the different generations. The Lagrangian is therefore not expressed in terms of fields of definite mass but rather the eigenstates of the interactions. Redefining the fields using unitarity transformations ${ }^{2}$

$$
\begin{array}{ll}
u_{L}=L_{u} u_{L}^{\prime} & , \quad u_{R}=R_{u} u_{R}^{\prime}, \\
d_{L}=L_{d} d_{L}^{\prime} & , \quad d_{R}=R_{d} d_{R}^{\prime}, \\
e_{L}=L_{e} e_{L}^{\prime} & , \quad e_{R}=R_{e} e_{R}^{\prime}
\end{array}
$$

so that $D_{k}=L_{k}^{\dagger} M_{k} R_{k}$ is diagonal with positive entries, allows rewriting the Lagrangian in terms of the mass eigenstates. The consequence of this redefinition in the terms of the Lagrangian is minimal. The kinetic terms and the $Z$ and $A$ couplings are unaffected by the unitary transformations. Also since neutrinos are assumed to be massless, the lepton fields may be chosen to be simultaneous mass and weak eigenstates, so lepton terms are unaffected as well. The only change in the Lagrangian is in the quark couplings with the $W$ bosons, which only interact with the left-handed fermions. Defining $L_{u}^{\dagger} L_{d}$ as the Cabibbo-Kobayashi-Maskawa (CKM) matrix $V_{\mathrm{CKM}}$, these terms become

$$
\begin{aligned}
\mathcal{L}_{W^{ \pm}} & =-\frac{g_{2}}{\sqrt{2}} \bar{u}_{L}^{i} \gamma^{\mu} d_{L}^{i} W_{\mu}^{+}+\text {h.c. } \\
& =-\frac{g_{2}}{\sqrt{2}} \bar{u}^{\prime}{ }_{L}^{i} \gamma^{\mu}\left(V_{\mathrm{CKM}}\right)^{i j}{d^{\prime}}_{L}^{j} W_{\mu}^{+}+\text {h.c. }
\end{aligned}
$$

The flavor mixing introduced by the Yukawa interactions is now encapsulated in

$$
V_{\mathrm{CKM}}=\left(\begin{array}{ccc}
V_{u d} & V_{u s} & V_{u b} \\
V_{c d} & V_{c s} & V_{c b} \\
V_{t d} & V_{t s} & V_{t b}
\end{array}\right)
$$

\footnotetext{
${ }^{2}$ The convention presented here transforms both the up and down quark fields. We may also choose to only transform one set of these fields.
} 
and mediated by the $W$ boson exchanges of the left-handed quarks.

\subsubsection{CP Violation in the Standard Model}

In order to describe observations, the $\mathrm{SM}$ must exhibit $\mathrm{C}, \mathrm{P}$, and $\mathrm{CP}$ violations. $\mathrm{A}$ quick glance at table 2.3 reveals that $\mathrm{P}$ and $\mathrm{C}$ violations were built into our construction of the SM through the absence of the right-handed neutrino and the lack of $S U(2)$ charge for the right-handed fermions. Hence these asymmetries are the consequence of the first building block (see section 2.2) of the SM. Finding CP Violation, however, requires a more detailed inspection of the model.

Noting that pure gauge Lagrangians are necessarily CP-invariant [8], eliminates the second building block, the gauge symmetries, as a source of CPV. Hence we are left with the Higgs mechanism. The scalar potential of one Higgs doublet clearly conserves $\mathrm{CP}^{3}$. Therefore, the only remaining element of $\mathrm{SM}$ is the Yukawa interactions of the fermions with the Higgs. However, since the these couplings were explicitly diagonalized and the fermion field redefined to produce mass terms, CP violation is not apparent in the interactions with the Higgs. Recalling that the affect of this redefinition was the addition of $V_{i j}$ to the flavor changing interactions, we may then identify the CKM as the only potential source of CP violation.

Examining the relevant terms in the SM Lagrangian, we note that

$$
(C P) \frac{g_{2}}{\sqrt{2}} \bar{u}_{L}^{\prime}{ }^{i} \gamma^{\mu}\left(V_{\mathrm{CKM}}\right)^{i j} d_{L}^{\prime j} W_{\mu}^{+}(C P)^{\dagger}=e^{i \phi} \frac{g_{2}}{\sqrt{2}} \bar{d}_{L}^{\prime}{ }_{L} \gamma^{\mu}\left(V_{\mathrm{CKM}}\right)^{i j} u_{L}^{\prime j} W_{\mu}^{-}
$$

which when compared to the hermitian conjugate term $\frac{g_{2}}{\sqrt{2}} \bar{d}_{L}^{i} \gamma^{\mu}\left(V_{\mathrm{CKM}}^{*}\right)^{i j} d_{L}^{j} W_{\mu}^{-}$implies that $\mathrm{CP}$ conservation requires

$$
V_{i j}^{*}=e^{i \phi} V_{i j}
$$

\footnotetext{
${ }^{3}$ More than one Higgs bosons may lead more sources of CPV.
} 
Here $\phi$ is an arbitrary single phase, which may be chosen to satisfy the condition (2.1) for one CKM element. However, the condition is not necessarily satisfied for all elements, and if more than one element of the CKM matrix is complex, CP conservation is violated in the SM.

Another potential source of $\mathrm{CP}$ violation in the $\mathrm{SM}$ is the likely presence of a $\mathrm{P}$ and $\mathrm{T}$ violating term

$$
\mathcal{L}_{\theta}=\bar{\theta} \frac{g_{s}^{2}}{32 \pi^{2}} F^{a \mu \nu} \tilde{F}_{\mu \nu}^{a}
$$

in the QCD Lagrangian. The origin of this effect, known as "the strong CP problem", are beyond the scope of the present discussion. The reader may refer to [9][p. 329] for details regarding this topic. Measurements of the electric dipole moment of the neutron, $D_{n}$, constrain $\bar{\theta}<3 \times 10^{-10}$ [10], which is significantly smaller than the observed CPV parameter in the CKM matrix.

\subsubsection{The CKM Matrix and Unitarity}

The unitarity of the CKM matrix may be viewed as a consequence of its definition as $V_{\mathrm{CKM}} \equiv L_{u}^{\dagger} L_{d}$, where the matrices $L_{u}$ and $L_{d}$ are required to be unitary so that $\left|\bar{u}_{L} u_{L}\right|=\left|\bar{u}_{L}^{\prime} u_{L}^{\prime}\right|$ and $\left|\bar{d}_{L} d_{L}\right|=\left|\bar{d}_{L}^{\prime} d_{L}^{\prime}\right|^{4}$. Since this imposes strong requirements on CKM elements, we quickly check that CPV through complex elements in CKM matrix is permitted.

Unitarity imposes the 9 constraints

$$
\sum_{i} V_{i j}^{*} V_{i k}=\sum_{i} V_{i j} V_{i k}^{*}=\delta_{j k}
$$

on the matrix's 18 (9 real +9 complex) parameters, eliminating 9 of them. Since the matrix only appears in the Lagrangian contracted with two different fields (i.e.

\footnotetext{
${ }^{4}$ Unitarity also insures positive masses for the fermions.
} 
$\left.\bar{u}_{L}^{i} \gamma^{\mu} V^{i j} d_{L}^{j}\right)$, five phases may be absorbed into $u_{L}^{i}$ and $d_{L}^{i}$. Furthermore, the remaining 4 parameters can be chosen such that only one is complex. This parameter is the solitary source of $\mathrm{CP}$ violation in the quark sector of the SM. It is important to note that in the accounting performed here, three quark-lepton generations are the minimal requirement for a complex parameter in the CKM matrix (i.e. two generations would result in no CPV phases).

A survey of measurements of the magnitudes of CKM elements provide [11]:

$$
\left|V_{\mathrm{CKM}}\right|=\left(\begin{array}{ccc}
0.97504 \pm 0.00049 & 0.2221 \pm 0.0021 & 0.00270-0.00371 \\
0.2220 \pm 0.0021 & 0.97414 \pm 0.00049 & 0.00387-0.00432 \\
0.0072-0.0092 & 0.0380-0.0427 & 0.99907-0.99926
\end{array}\right)
$$

We observe that transitions within each quark-lepton family is much more probable than those between the first and second, which are more probable than second and third, leaving transitions between the first and third generations as the least likely. A convenient approximate parameterization of the CKM was suggested by Wolfenstein $[12]^{5}$ :

$$
V_{\mathrm{CKM}}=\left(\begin{array}{ccc}
1-\frac{\lambda^{2}}{2} & \lambda & A \lambda^{3}(\rho-i \eta) \\
-\lambda & 1-\frac{\lambda^{2}}{2} & A \lambda^{2} \\
A \lambda^{3}(1-\rho-i \eta) & -A \lambda^{2} & 1
\end{array}\right)+O\left(\lambda^{4}\right) .
$$

The measured quantities in 2.3 reflect that $\left|V_{u s}\right|$ and $\left|V_{c b}\right|$ (and therefore $\lambda$ and $A$ ) can be measure directly and are well known. Current measurements of $\left|V_{u b}\right|$ and $\left|V_{t d}\right|$ (therefore $\rho$, and $\eta$ ), however, have large uncertainties [24].

\footnotetext{
${ }^{5}$ For an exact parameterization of the CKM matrix in terms of 3 real parameters and 1 complex phase, please see [13].
} 


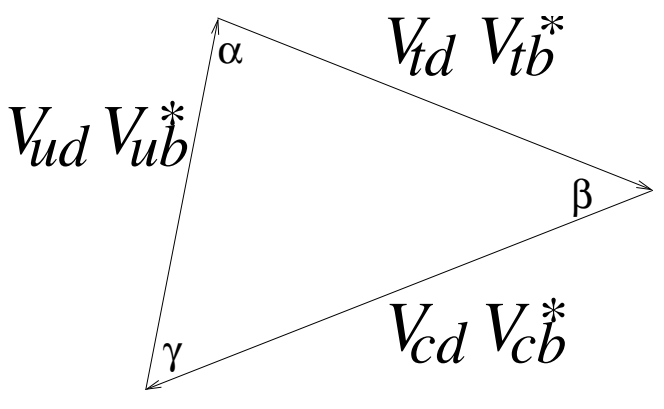

Figure 2.1: The Unitarity Triangle.

\subsubsection{The Unitarity Triangle}

The unitarity conditions (2.2) relate the nine complex elements $V_{i j}$ of the CKM matrix to one another. Since these elements are in principle all directly measurable, the consistency of the unitarity conditions may be experimentally confirmed. Any evidence of the failure of the unitarity of the CKM matrix is an indication of one of two possibilities: there are more than three generations of quarks and leptons, or there is new physics beyond the CKM picture of flavor changing interactions. The former possibility is constrained by measurements of the number of light neutrino flavors contributing to $Z$ decays. Therefore discovering that the CKM elements do not obey the unitarity conditions is a good indication of new physics.

The equations (2.2) form six triangles and three rectangles in the complex plane, providing convenient geometric representations of the unitarity conditions. In particular, the relation containing all $b$ quark elements,

$$
V_{u b}^{*} V_{u d}+V_{c b}^{*} V_{c d}+V_{t b}^{*} V_{t d}=0,
$$

is often used to form the Unitarity Triangle (UT) which is pictured in figure 2.1. Here the resulting angles

$$
\alpha=\arg \left[-\frac{V_{t d} V_{t b}^{*}}{V_{u d} V_{u b}^{*}}\right], \beta=\arg \left[-\frac{V_{c d} V_{c b}^{*}}{V_{t d} V_{t b}^{*}}\right], \gamma=\arg \left[-\frac{V_{u d} V_{u b}^{*}}{V_{c d} V_{c b}^{*}}\right],
$$




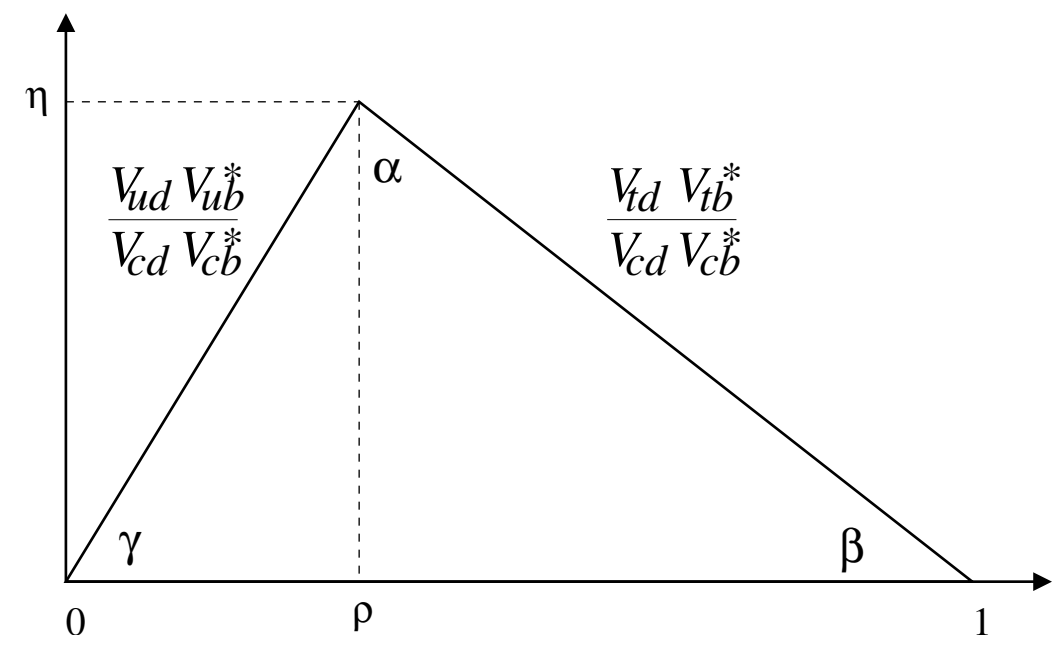

Figure 2.2: The Unitarity Triangle in the $\rho-\eta$ plane.

are sensitive to $B$ meson decays to specific final states and are in principle experimentally measurable. Confirming that $\alpha+\beta+\gamma=\pi$ is a test of the unitarity of the CKM matrix.

Recalling that unitarity implies that the CKM matrix is composed of only 4 parameters, we use the Wolfenstein parameterization to represent the UT in terms of the least well known parameters, $\rho$ and $\eta$. The triangle in figure 2.2 is therefore constructed by choosing $V_{c d} V_{c b}^{*}$ to be real and scaling the sides of the triangle by $V_{c d} V_{c b}^{*}$. This representation of the UT provides a convenient visual means of understanding and comparing experimental measurements.

\subsection{CP Violation Phenomenology}

Having identified the source of CPV in the SM, we now turn our attention to how to observe CPV phenomena in decays of mesons. The elusiveness of this task may be inferred from the 37 years between the discovery of CPV in the kaon and $B$ meson 
systems.

\subsubsection{Strong and Weak Phases}

Consider the transition from the states $i$ and $\bar{i}$ to final states $f$ and $\bar{f}$ with only one amplitude contributing:

$$
\begin{aligned}
& \langle f|T| i\rangle=A e^{i(\delta+\phi)}, \\
& \langle\bar{f}|T| \bar{i}\rangle=A e^{i(\delta-\phi)},
\end{aligned}
$$

where $T$ is the transition operator and $A$ is a positive real number. The CP-even phase that is common to both decays, $\delta$, is referred to as a strong phase, and the CPodd phase that changes signs, $\phi$, is referred to as a weak phase ${ }^{6}$. The CP operator relates the $\mathrm{CP}$ conjugate states by inducing arbitrary phases:

$$
\begin{gathered}
C P|i\rangle=e^{i \eta_{i}}|\bar{i}\rangle \quad, \quad C P|\bar{i}\rangle=e^{-i \eta_{i}}|i\rangle, \\
C P|f\rangle=e^{i \eta_{f}}|\bar{f}\rangle \quad, \quad C P|\bar{f}\rangle=e^{-i \eta_{f}}|f\rangle .
\end{gathered}
$$

If $\mathrm{CP}$ is conserved by $T$,

$$
\langle f|T| i\rangle=\left\langle f\left|(C P) T(C P)^{\dagger}\right| i\right\rangle=e^{i\left(\eta_{i}-\eta_{f}\right)}\langle\bar{f}|T| \bar{i}\rangle .
$$

Choosing $\eta_{i}-\eta_{f}=2 \phi-\theta$, we see that despite the presence of the CP violating phase $\phi$ in this transition, the observable amplitudes are incapable of indicating any CP violation in $T$.

$\mathrm{CP}$ violation is observable in transitions with two strong and weak phase contri-

\footnotetext{
${ }^{6}$ In general we could also add a spurious phase betweeen the two states which would have no influence on this discussion. See [9].
} 
butions. Consider

$$
\begin{aligned}
\langle f|T| i\rangle & =A_{1} e^{i\left(\delta_{1}+\phi_{1}\right)}+A_{2} e^{i\left(\delta_{2}+\phi_{2}\right)}, \\
\langle\bar{f}|T| \bar{i}\rangle & =A_{1} e^{i\left(\delta_{1}-\phi_{1}+\theta\right)}+A_{2} e^{i\left(\delta_{2}-\phi_{2}+\theta\right)} .
\end{aligned}
$$

Here the presence of interference between the two amplitudes allows the construction of the $\mathrm{CP}$ violating observable

$$
|\langle f|T| i\rangle|^{2}-|\langle\bar{f}|T| \bar{i}\rangle|^{2}=-4 A_{1} A_{2} \sin \left(\delta_{1}-\delta_{2}\right) \sin \left(\phi_{1}-\phi_{2}\right) .
$$

Note however, that in order to obtain CPV, at least two differing strong and weak phases are necessary. Such expression of CPV is known as direct CP violation.

It is possible to obtain $\mathrm{CP}$ violating observables without strong phases when considering decays to two different final states, or when $i$ and $\bar{i}$ decay to the same final state $f=\bar{f}$. We'll consider this latter case in the discussions that follow.

\subsubsection{Neutral Mesons}

In the absence of the weak interaction, a $P^{0}$ meson such as $K^{0}, D^{0}$, or $B^{0}$ would be stable and have a common mass with $\bar{P}^{0}$. Weak transitions, however, permit $P^{0} \leftrightarrow \bar{P}^{0}$ mixing, forming mass/lifetime eigenstates which are a mixure of the flavor eigenstates. Under the Wigner-Weisskopf approximation [14], the Schroedinger equation for the time evolution and decay of the meson system

$$
|\psi(t)\rangle=\psi_{1}(t)\left|P^{0}\right\rangle+\psi_{2}(t)\left|\bar{P}^{0}\right\rangle
$$

may be written in the $\left|P^{0}\right\rangle /\left|\bar{P}^{0}\right\rangle$ basis as

$$
i \frac{d}{d t}\left(\begin{array}{c}
\psi_{1} \\
\psi_{2}
\end{array}\right)=\mathcal{H}\left(\begin{array}{c}
\psi_{1} \\
\psi_{2}
\end{array}\right)=\left(\mathbf{M}-\frac{i}{2} \boldsymbol{\Gamma}\right)\left(\begin{array}{c}
\psi_{1} \\
\psi_{2}
\end{array}\right) .
$$


Here the matrices $\mathbf{M}$ and $\boldsymbol{\Gamma}$ may be computed from the weak Hamiltonian $\mathcal{H}_{\mathrm{EW}}$ in second-order perturbation theory as

$$
\begin{aligned}
M_{i j} & =m_{0} \delta_{i j}+\left\langle i\left|\mathcal{H}_{\mathrm{EW}}\right| j\right\rangle+\sum_{n} P \frac{\left\langle i\left|\mathcal{H}_{\mathrm{EW}}\right| n\right\rangle\left\langle n\left|\mathcal{H}_{\mathrm{EW}}\right| j\right\rangle}{m_{0}-E_{n}}, \\
\Gamma_{i j} & =2 \pi \sum_{n} \delta\left(m_{0}-E_{n}\right)\left\langle i\left|\mathcal{H}_{\mathrm{EW}}\right| n\right\rangle\left\langle n\left|\mathcal{H}_{\mathrm{EW}}\right| j\right\rangle .
\end{aligned}
$$

Since the "Hamiltonian" $\mathcal{H}$ in (2.6) is not hermitian, its eigenstates?

$$
\begin{aligned}
& \left|P_{L}\right\rangle=p\left|P^{0}\right\rangle+q\left|\bar{P}^{0}\right\rangle, \\
& \left|P_{H}\right\rangle=p\left|P^{0}\right\rangle-q\left|\bar{P}^{0}\right\rangle,
\end{aligned}
$$

have the complex eigenvalues given by

$$
\Delta \mu \equiv \Delta m-\frac{i}{2} \Delta \Gamma=\left(m_{H}-m_{L}\right)-\frac{i}{2}\left(\Gamma_{H}-\Gamma_{L}\right)=2 \sqrt{\mathcal{H}_{12} \mathcal{H}_{21}},
$$

where $\left(m_{H}, m_{L}\right)$ and $\left(\Gamma_{H}^{-1}, \Gamma_{L}^{-1}\right)$ are the masses and lifetimes of the heavy and light states $\left|P_{H}\right\rangle$ and $\left|P_{L}\right\rangle$. The ratio

$$
\frac{p}{q}=\frac{\Delta \mu}{2 M_{12}-i \Gamma_{12}}=\frac{2 M_{12}^{*}-i \Gamma_{12}^{*}}{\Delta \mu}=\sqrt{\frac{2 M_{12}^{*}-i \Gamma_{12}^{*}}{2 M_{12}-i \Gamma_{12}}}
$$

will indicate if $\mathrm{CP}$ is violated in mixing of $P^{0}$ and $\bar{P}^{0}$. Using

$$
\begin{aligned}
& C P\left|P^{0}\right\rangle=e^{i \eta}\left|\bar{P}^{0}\right\rangle, \\
& C P\left|\bar{P}^{0}\right\rangle=e^{-i \eta}\left|P^{0}\right\rangle,
\end{aligned}
$$

we find that requiring the conditions 2.4 in equation 2.7 implies that $\mathrm{CP}$ is conserved when

$$
\begin{aligned}
& M_{12}^{*}=e^{2 i \eta} M_{12}, \\
& \Gamma_{12}^{*}=e^{2 i \eta} \Gamma_{12},
\end{aligned}
$$

${ }^{7} \mathrm{CPT}$ invariance is employed here to allow the same $p$ and $q$ to be used for $\left|P_{L}\right\rangle$ and $\left|P_{H}\right\rangle$ and $\mathcal{H}_{22}=\mathcal{H}_{11}$. 
or rather

$$
\frac{p}{q}= \pm e^{i \eta} \Rightarrow\left|\frac{p}{q}\right|=1 .
$$

As expected, this condition shows that $\mathrm{CP}$ invariance implies that $\left|P_{L}\right\rangle$ and $\left|P_{H}\right\rangle$ are $\mathrm{CP}$ eigenstates. Failure of this condition indicates $C P$ violation in mixing or indirect CP violation.

\subsubsection{CP Violating Observables}

Consider the decay of the $P^{0} / \bar{P}^{0}$ meson to the final states $f / \bar{f}$ :

$$
\begin{array}{ll}
A_{f} \equiv\left\langle f|T| P^{0}\right\rangle & , \quad \bar{A}_{f} \equiv\left\langle f|T| \bar{P}^{0}\right\rangle, \\
A_{\bar{f}} \equiv\left\langle\bar{f}|T| P^{0}\right\rangle \quad, \quad \bar{A}_{\bar{f}} \equiv\left\langle\bar{f}|T| \bar{P}^{0}\right\rangle .
\end{array}
$$

Applying (2.4) and (2.11) to these amplitudes leads to the CP-invariance conditions:

$$
\begin{aligned}
& \bar{A}_{\bar{f}}=e^{i\left(\eta_{f}-\eta\right)} A_{f} \Rightarrow\left|A_{f}\right|=\left|\bar{A}_{\bar{f}}\right|, \\
& A_{\bar{f}}=e^{i\left(\eta_{f}+\eta\right)} \bar{A}_{f} \Rightarrow\left|A_{\bar{f}}\right|=\left|\bar{A}_{f}\right| .
\end{aligned}
$$

As expected, the decay probabilities for $P^{0}$ to $f$ and $\bar{P}^{0}$ to $\bar{f}$ must be the same to conserve CP. Deviation from these conditions signifies $C P$ violation in decay.

We may construct a more concise $\mathrm{CP}$ conservation requirement by combining the individual conditions for mixing and decay. Taking the ratio of the conditions (2.14) and $(2.15)$ we find

$$
\frac{A_{f} A_{\bar{f}}}{\bar{A}_{f} \bar{A}_{\bar{f}}}=e^{2 i \eta}=\frac{q^{2}}{p^{2}} .
$$

Defining

$$
\Lambda_{f} \equiv \frac{q}{p} \frac{\bar{A}_{f}}{A_{f}}, \Lambda_{\bar{f}} \equiv \frac{q}{p} \frac{\bar{A}_{\bar{f}}}{A_{\bar{f}}},
$$

allows (2.16) to be written more simply as

$$
\Lambda_{f}=\frac{1}{\Lambda_{\bar{f}}} .
$$


This condition encapsulates another possible expression of $\mathrm{CP}$ violation. In order to illustrate, let us consider the simplified case when $P^{0}$ and $\bar{P}^{0}$ decay to a CP eigenstate (i.e. $C P|f\rangle=\eta_{f_{C P}}|f\rangle, \eta_{f_{C P}}= \pm 1$ ), and there is no CPV mixing or decay:

$$
\begin{aligned}
A_{f}=A e^{i\left(\delta+\phi_{D}\right)}, \bar{A}_{f}=\eta_{f_{C P}} A e^{i\left(\delta-\phi_{D}\right)} & \Rightarrow\left|A_{f}\right|=\left|\bar{A}_{f}\right| \\
q / p=e^{2 i \phi_{M}} & \Rightarrow|q / p|=1 .
\end{aligned}
$$

As before, we have introduced a strong phase but we have used different mixing and decay weak phases $\phi_{M}$ and $\phi_{D}$. In this case, $\Lambda_{f}=\eta_{f_{C P}} e^{2 i\left(\phi_{M}-\phi_{D}\right)}$. However, since $f=\bar{f}, \Lambda_{f}=\Lambda_{\bar{f}}$, and (2.17) becomes

$$
\Lambda_{f}= \pm 1=\eta_{f_{C P}} e^{2 i\left(\phi_{M}-\phi_{D}\right)}
$$

and a less apparent expression of CPV is revealed: CP violation in interference between mixing and decay.

In the case of meson decays to $\mathrm{CP}$ eigenstates, $\Lambda \neq \pm 1$ for any of the three types of $\mathrm{CP}$ violation: $\mathrm{CP}$ violation in mixing, $|q / p| \neq 1$; CP violation in decay, $\left|\bar{A}_{f} / A_{f}\right| \neq 1$; and $\mathrm{CP}$ violation in interference between mixing and decay, non-vanishing relative phase between $q / p$ and $\bar{A}_{f} / A_{f}$. In the next section we will see how $\Lambda_{f}$ appears in the time-evolution of neutral mesons, specifically focusing on the $B^{0}$. We will also see how $\Lambda_{f}$ is directly related to CKM parameters for specific $B$ decays.

\subsection{Neutral $B$ Mesons}

We now turn our attention to the $B$ factories, where large numbers of $B$ meson pairs are produced through the process $e^{+} e^{-} \rightarrow \Upsilon(4 s) \rightarrow B \bar{B}$. To a very good

approximation half of these pairs are the neutral $B^{0} / \bar{B}^{0} \mathrm{~s}$. Studies of the decay of 


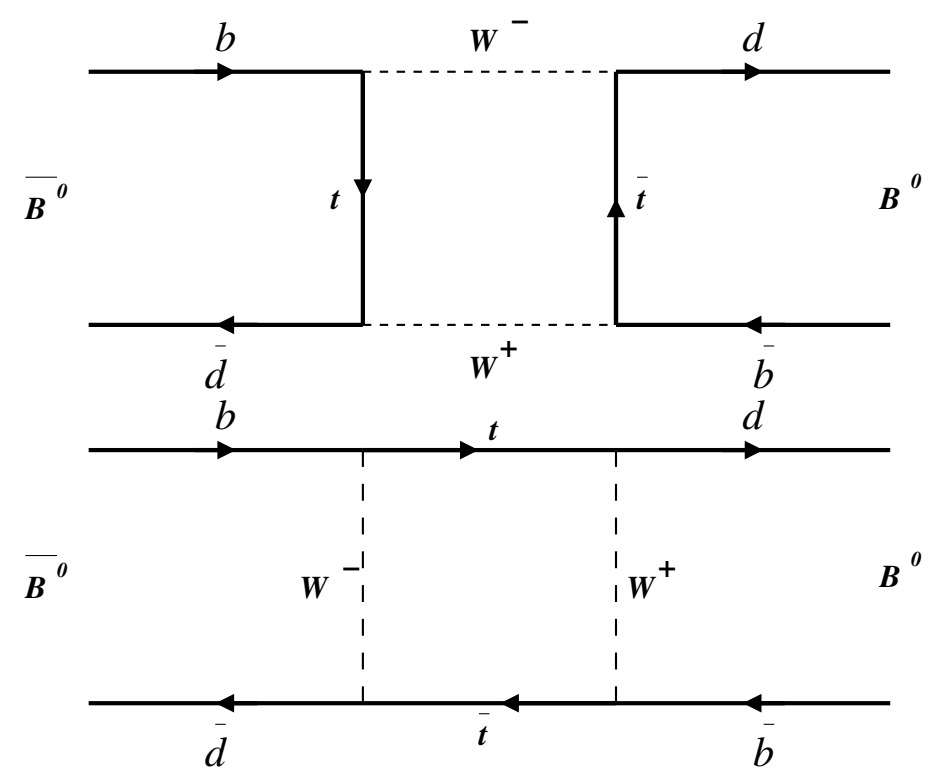

Figure 2.3: The leading diagrams contributing to $B^{0}-\bar{B}^{0}$ mixing.

these mesons to CP eigenstates provides a means of measuring angles of the unitarity triangle.

\subsubsection{Time Evolution of $B$ 's}

After production, a solitary $B^{0}$ (or $\bar{B}^{0}$ ) will evolve according to the Schroedinger equation (2.6). Before decaying, the meson may change its flavor several times through the box diagrams in figure 2.3. The time-dependent mass eigenstates

$$
\begin{aligned}
\left|B_{L}(t)\right\rangle & =e^{-i m_{L} t} e^{-\Gamma_{L} t / 2}\left|B_{L}\right\rangle, \\
\left|B_{H}(t)\right\rangle & =e^{-i m_{H} t} e^{-\Gamma_{H} t / 2}\left|B_{H}\right\rangle,
\end{aligned}
$$


are related to the flavor eigenstates by (2.9). Therefore

$$
\begin{aligned}
&\left|B^{0}(t)\right\rangle=\left(e^{-\left(i m_{H}+\Gamma_{H} / 2\right) t}+e^{-\left(i m_{L}+\Gamma_{L} / 2\right) t}\right)\left|B^{0}\right\rangle+ \\
& \frac{q}{p}\left(e^{-i\left(m_{H}+\Gamma_{H} / 2\right) t}-e^{-i\left(m_{L}+\Gamma_{L} / 2\right) t}\right)\left|\bar{B}^{0}\right\rangle, \\
&\left|\bar{B}^{0}(t)\right\rangle=\frac{q}{p}\left(e^{-i\left(m_{H}+\Gamma_{H} / 2\right) t}-e^{-i\left(m_{L}+\Gamma_{L} / 2\right) t}\right)\left|B^{0}\right\rangle+ \\
&\left(e^{-i\left(m_{H}+\Gamma_{H} / 2\right) t}+e^{-i\left(m_{L}+\Gamma_{L} / 2\right) t}\right)\left|\bar{B}^{0}\right\rangle .
\end{aligned}
$$

$\Upsilon(4 s)$ [19] decay, however, will produce two neutral $B$ mesons in a coherent antisymmetric state. This two meson system will consist of one $B$ of each flavor until one particle decays. From that time on, the remaining $B$ will obey (2.21) until its decay. If one meson decays to a CP eigenstate, there is no means of identifying its flavor. We will refer to this meson as $B_{C P}$ with decay time $t_{C P}$. However, since at time of the first decay only one meson of each flavor was present, the flavor of $B_{C P}$ may be inferred from the other meson. We will refer to this meson as $B_{\text {tag }}$ with decay time

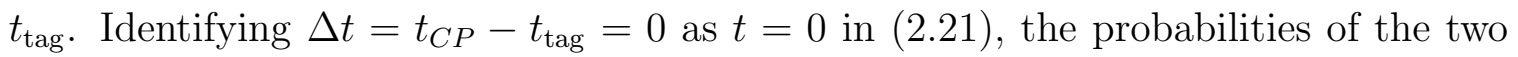
observable anti-symmetric states (i.e. when $B_{\operatorname{tag}}$ is a $B^{0}$ or $\bar{B}^{0}$ ) are

$$
\begin{aligned}
\Gamma_{B^{0}}(\Delta t)= & \frac{1}{2} \mid\left\langle f|T| B^{0}\left(t=t_{C P}\right)\right\rangle\left\langle B^{0}\left(t=t_{\mathrm{tag}}\right) \mid \bar{B}^{0}\left(t=t_{\mathrm{tag}}\right)\right\rangle- \\
\left.\left\langle f|T| \bar{B}^{0}\left(t=t_{C P}\right)\right\rangle\left\langle B^{0}\left(t=t_{\mathrm{tag}}\right) \mid B^{0}\left(t=t_{\mathrm{tag}}\right)\right\rangle\right|^{2} & \frac{e^{-\frac{|\Delta t|}{\tau}}}{4 \tau}\left(1+S_{f} \sin \left(\Delta m_{d} \Delta t\right)-C_{f} \cos \left(\Delta m_{d} \Delta t\right)\right), \\
\Gamma_{\bar{B}^{0}}(\Delta t)= & \frac{1}{2} \mid\left\langle f|T| B^{0}\left(t=t_{C P}\right)\right\rangle\left\langle\bar{B}^{0}\left(t=t_{\mathrm{tag}}\right) \mid \bar{B}^{0}\left(t=t_{\mathrm{tag}}\right)\right\rangle- \\
& \left.\left\langle f|T| \bar{B}^{0}\left(t=t_{C P}\right)\right\rangle\left\langle\bar{B}^{0}\left(t=t_{\mathrm{tag}}\right) \mid B^{0}\left(t=t_{\mathrm{tag}}\right)\right\rangle\right|^{2} \\
= & \frac{e^{-\frac{|\Delta t|}{\tau}}}{4 \tau}\left(1-S_{f} \sin \left(\Delta m_{d} \Delta t\right)+C_{f} \cos \left(\Delta m_{d} \Delta t\right)\right),
\end{aligned}
$$

where $\Delta m_{d}$ is the mass difference between $B_{L}$ and $B_{H}$ and the lifetime difference is 
assumed to be negligible ${ }^{8}$. Here

$$
S_{f}=\frac{2 \operatorname{Im} \Lambda_{f}}{1+\left|\Lambda_{f}^{2}\right|} \text { and } C_{f}=\frac{1-\left|\Lambda_{f}^{2}\right|}{1+\left|\Lambda_{f}^{2}\right|} .
$$

As before,

$$
\Lambda_{f}=\eta_{f_{C P}} \frac{p}{q} \frac{\overline{A_{f}}}{A_{f}},
$$

where $A=\left|\left\langle f|T| B^{0}\right\rangle\right|, \bar{A}=\left|\left\langle f|T| \bar{B}^{0}\right\rangle\right|$, and $\eta_{f_{C P}}$ is the CP eigenvalue of the final state.

Equations 2.23 and 2.24 illustrate that the time structure of $B$ decays to CP eigenstates depend on $\Lambda_{f}$, providing an experimental means of accessing the observable which is sensitive to the three types of CPV. As an example, consider the variable

$$
\begin{aligned}
A_{C P}(\Delta t) & =\frac{\Gamma_{B^{0}}(\Delta t)-\Gamma_{\overline{B^{0}}}(\Delta t)}{\Gamma_{B^{0}}(\Delta t)+\Gamma_{\bar{B}^{0}}(\Delta t)} \\
& =S \sin \left(\Delta m_{d} \Delta t\right)-C \cos \left(\Delta m_{d} \Delta t\right),
\end{aligned}
$$

under the conditions (2.18) and (2.19), i.e. no CP violation in mixing or in decay. Here $A_{C P}(t)$ will exhibit the oscillatory behavior

$$
A_{C P}(\Delta t)=\eta_{f_{C P}} \sin 2\left(\phi_{M}-\phi_{D}\right) \sin \left(\Delta m_{d} \Delta t\right)
$$

with frequency $\Delta m_{d}$ and an amplitude which is related to the phase difference $\phi_{D}-\phi_{M}$ between the mixing and decay factors in $\Lambda_{f}$. A non-zero amplitude indicates $\mathrm{CP}$ violation in interference between mixing and decay. Measuring $A_{C P}$, however, requires a sophisticated analysis of the $\Upsilon(4 s)$ decay where one $B$ meson is identified to decay to a $\mathrm{CP}$ eigenstate, the flavor of the other $B$ meson is determined, and difference between the decay times of the two mesons is measured. We will detail the specific requirements of such an analysis in later chapters.

${ }^{8} \Delta \Gamma$ is sensitive to $\Gamma_{12}$, which (2.7) shows is small for mesons where only a small fraction of decays are accessible to $B^{0}$ and $\bar{B}^{0}$. $B$ mesons are an example. See [19] for more details. 

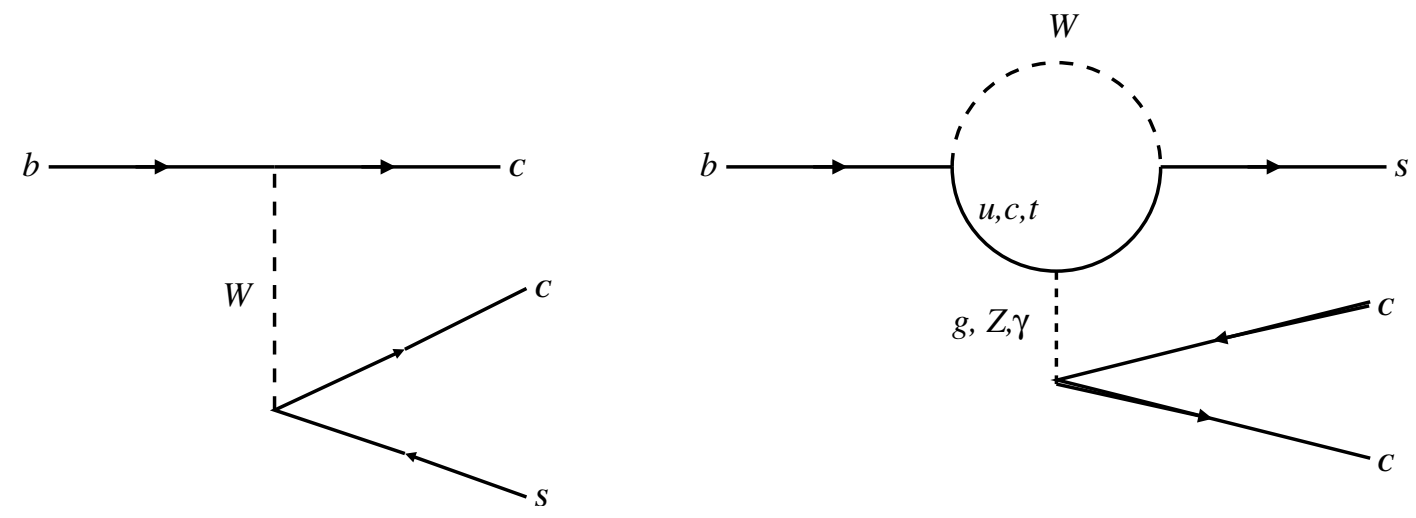

Figure 2.4: $B \rightarrow J / \psi K_{s}$ tree and penguin diagrams.

\subsubsection{Relating $\Lambda_{f}$ to the CKM matrix}

In general, the SM amplitudes for $B$ decays may carry contributions from multiple Feynman diagrams, each carry different CKM matrix elements. Therefore the amplitude ratio in $\Lambda_{f}$ is of the form:

$$
\frac{\overline{A_{f}}}{A_{f}}=\frac{A_{f}^{\alpha} e^{i \alpha}+A_{f}^{\beta} e^{i \beta}+A_{f}^{\gamma} e^{i \gamma}+\ldots}{A_{f}^{\alpha} e^{-i \alpha}+A_{f}^{\beta} e^{-i \beta}+A_{f}^{\gamma} e^{-i \gamma}+\ldots} .
$$

If all of the amplitudes contributing to $A$ and $\bar{A}$ could be calculated for a given decay, $\Lambda_{f}$ 's relation to CKM matrix elements and unitary triangle angles would be easy to identify. Unfortunately calculating amplitudes for hadronic $B$ decays is rather complex $[28,31]$. Though the short distance processes governed by the weak interaction and hard QCD can be cleanly calculated, long distance processes like hadronization and rescattering are difficult.

Decays dominated by one phase require no hadronic calculation. As an example, consider the leading diagrams for the decay $B \rightarrow J / \psi K_{s}$ presented figure 2.4. To highest order in the Wolfenstein parameter $\lambda$, the tree level diagram containing the factors $V_{c b} V_{c s}^{*} \approx \lambda^{2}$ and the leading loop diagram (known as penguin diagram) con- 
taining the factor $V_{t b} V_{t s}^{*} \approx \lambda^{2}+O\left(\lambda^{4}\right) e^{-i \gamma}$ carry the same CKM phase [18]. Therefore

$$
\begin{aligned}
\Lambda_{J / \psi K_{s}} & =-\frac{V_{t d} V_{t b}^{*}}{V_{t b} V_{t d}^{*}} \frac{V_{c b} V_{c s}^{*}}{V_{c s} V_{c b}^{*}} \frac{V_{c d} V_{c d}^{*}}{V_{c d} V_{c s}^{*}} \\
\Rightarrow \operatorname{Im} \Lambda_{J / \psi K_{s}} & =\sin 2 \beta,
\end{aligned}
$$

where the first term is $q / p$ (from diagrams in figure 2.3), the last term comes from $K^{0}-\bar{K}^{0}$ mixing, and the middle term is $\bar{A} / A$. Since $\Lambda_{J / \psi K_{s}}$ is so cleanly related to the angle $\beta$, this decay of the $B$ meson is often referred to as "the gold-plated mode".

\section{$2.5 \quad B^{0} \rightarrow \pi^{+} \pi^{-}, K^{+} \pi^{-}$}

The $B^{0}$ decay to the CP eigenstate $\pi^{+} \pi^{-}$is the simplest transition sensitive to the UT angle $\alpha$. Consider the tree level diagram in figure 2.5, which carries the CKM elements $V_{u b} V_{u d}^{*} \approx \lambda^{3} e^{-i \gamma}$. If $B^{0} \rightarrow \pi^{+} \pi^{-}$is dominated by the corresponding amplitude,

$$
\begin{aligned}
\Lambda_{\pi^{+} \pi^{-}} & =\frac{V_{t d} V_{t b}^{*}}{V_{t d} V_{t b}^{*}} \frac{V_{u d} V_{u b}^{*}}{V_{u b} V_{u d}^{*}} \\
\Rightarrow \operatorname{Im} \Lambda_{\pi^{+} \pi^{-}} & =\sin 2 \alpha .
\end{aligned}
$$

The dominant penguin contribution to this decay, the $t$-quark diagram shown in figure 2.6, carries the CKM elements $V_{t b} V_{t d}^{*} \approx \lambda^{3} e^{i \beta}$. Therefore, in terms of the ratio $R=P / T$ of the magnitudes (excluding CKM factors) of the penguin to tree amplitudes

$$
\begin{aligned}
\Lambda_{\pi^{+} \pi^{-}} & =\frac{V_{t d} V_{t b}^{*}}{V_{t d} V_{t b}^{*}} \frac{V_{u d} V_{u b}^{*}}{V_{u b} V_{u d}^{*}}\left[\frac{1+R\left(V_{t b} V_{t d}^{*}\right) /\left(V_{u b} V_{u d}^{*}\right)}{1+R\left(V_{t d} V_{t b}^{*}\right) /\left(V_{u d} V_{u b}^{*}\right)}\right] \\
& =e^{2 i \alpha} \frac{1-R e^{i \delta-\alpha}}{1-R e^{i \delta+\alpha}} \equiv\left|\Lambda_{\pi^{+} \pi^{-}}\right| e^{i 2 \alpha_{\mathrm{Eff}}}
\end{aligned}
$$

where $\delta$ is a relative strong phase between the two contributing amplitudes, and $\alpha_{\mathrm{Eff}}$ is the measurable "effective" angle. 
One indication that $R$ is non-negligible comes from the measured ratio $\frac{\mathcal{B}\left(B^{0} \rightarrow K^{+} \pi^{-}\right)}{\mathcal{B}\left(B^{0} \rightarrow \pi^{+} \pi^{-}\right)}$. The $b \rightarrow u \bar{u} s$ diagrams contributing to $B^{0} \rightarrow K^{+} \pi^{-}$are similar to the $b \rightarrow u \bar{u} d$ (see figures 2.5 and 2.6). If both decays are dominated by the tree amplitude, the ratio

$$
\frac{\mathcal{B}\left(B^{0} \rightarrow K^{+} \pi^{-}\right)}{\mathcal{B}\left(B^{0} \rightarrow \pi^{+} \pi^{-}\right)} \approx\left|\frac{V_{u b} V_{u s}^{*}}{V_{u b} V_{u d}^{*}}\right|^{2} \approx\left(\frac{\lambda^{4}}{\lambda^{3}}\right)^{2}=\lambda^{2},
$$

would be small. The measured world average of this ratio is $3.89 \pm .85$ [11], suggesting a large contribution from the penguin $b \rightarrow \bar{u} u s$ diagram which carries the CKM elements $V_{t b} V_{t s}^{*} \approx \lambda^{2}$. More precisely, recent estimates suggest $R \approx .2[15]$.

In addition to obscuring $\Lambda_{\pi^{+} \pi^{-}}$'s relation to $\alpha$, significant penguin contributions and non-zero strong phase $\delta$ leads to CPV in decay for both $B^{0} \rightarrow \pi^{+} \pi^{-}$and $B^{0} \rightarrow$ $K^{+} \pi^{-}$. Since in the latter case the flavor of the $B$ can be determined from the charge of the kaon, $B^{0} \rightarrow K^{+} \pi^{-}$is identical to the example presented earlier of a decay with two contributing amplitudes leading to (2.5). Therefore if the time averaged quantity

$$
A_{K \pi} \equiv \frac{\mathcal{B}\left(\bar{B}^{0} \rightarrow K^{-} \pi^{+}\right)-\mathcal{B}\left(B^{0} \rightarrow K^{+} \pi^{-}\right)}{\mathcal{B}\left(\bar{B}^{0} \rightarrow K^{-} \pi^{+}\right)+\mathcal{B}\left(B^{0} \rightarrow K^{+} \pi^{-}\right)}
$$

is not zero, CP is violated in $B^{0} \rightarrow K^{+} \pi^{-}$decays. For $B^{0} \rightarrow \pi^{+} \pi^{-}$, where the flavor of the decaying $B$ is not reconstructible, the cosine coefficient of the time-dependence,

$$
C_{\pi^{+} \pi^{-}}=\frac{\left|A_{\pi^{+} \pi^{-}}\right|^{2}-\left|\bar{A}_{\pi^{+} \pi^{-}}\right|^{2}}{\left|A_{\pi^{+} \pi^{-}}\right|^{2}+\left|\bar{A}_{\pi^{+} \pi^{-}}\right|^{2}}
$$

will be non-zero if $\mathrm{CP}$ is violated in decay.

In order to calculate $R$ and $\delta$, some degree of control and understanding of hadronic matrix elements is necessary. This problem is usually addressed by either relying heavily on several experimental measurements and the symmetries that relate them, or by focusing on first principle calculations and approximation techniques. Ultimately the validation of the theoretical approach also relies on experimental measurements. 


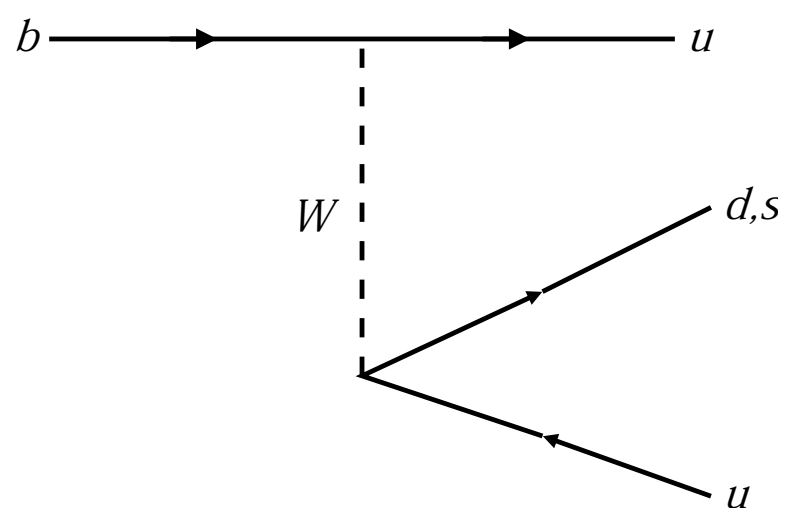

Figure 2.5: $B \rightarrow u \bar{u} d$ and $B \rightarrow u \bar{u} s$ tree diagrams.

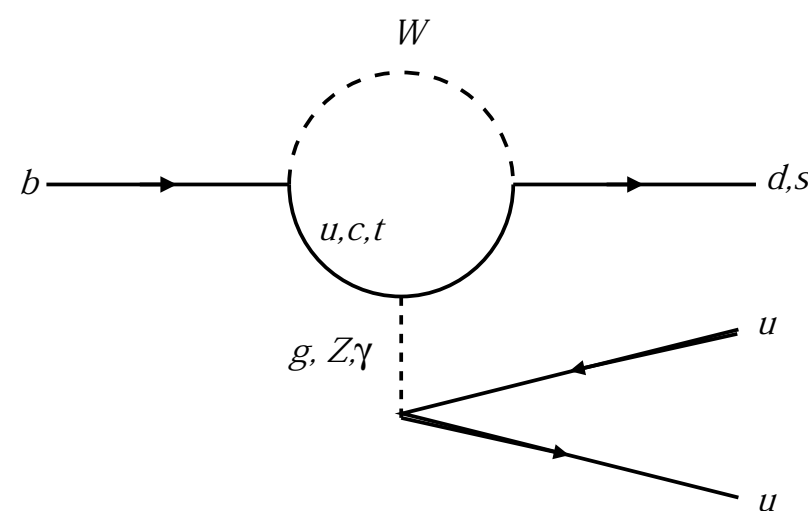

Figure 2.6: $B \rightarrow u \bar{u} d$ and $B \rightarrow u \bar{u} s$ penguin diagrams. 


\subsubsection{Disentangling Penguins and Trees using Isospin}

One suggested method of extracting $\alpha$ from $B \rightarrow \pi^{+} \pi^{-}$decays is to use isospin symmetry to relate the tree and penguin contributions to different two-pion $B$ decays [26]. Applying the Wigner-Eckart theorem to the decays from the initial isospin states $\left|B^{0}\right\rangle=\left|\frac{1}{2},-\frac{1}{2}\right\rangle$ and $\left|B^{+}\right\rangle=\left|\frac{1}{2}, \frac{1}{2}\right\rangle$, to the final states

$$
\begin{aligned}
\left|\pi^{0} \pi^{0}\right\rangle & =\sqrt{\frac{2}{3}}|2,0\rangle-\sqrt{\frac{1}{3}}|0,0\rangle, \\
\left|\pi^{+} \pi^{-}\right\rangle & =\sqrt{\frac{1}{3}}|2,0\rangle-\sqrt{\frac{2}{3}}|0,0\rangle, \\
\left|\pi^{+} \pi^{0}\right\rangle & =\sqrt{\frac{1}{2}}|2,1\rangle,
\end{aligned}
$$

indicates that transition amplitudes are restricted to be of the form

$$
\begin{aligned}
A^{+-} \equiv\left\langle\pi^{+} \pi^{-}|T| B^{0}\right\rangle & =-\sqrt{\frac{1}{3}} A_{1 / 2}+\sqrt{\frac{1}{6}} A_{3 / 2}-\sqrt{\frac{1}{6}} A_{5 / 2}, \\
A^{00} \equiv\left\langle\pi^{0} \pi^{0}|T| B^{0}\right\rangle & =\sqrt{\frac{1}{6}} A_{1 / 2}+\sqrt{\frac{1}{3}} A_{3 / 2}-\sqrt{\frac{1}{3}} A_{5 / 2}, \\
A^{+0} \equiv\left\langle\pi^{+} \pi^{0}|T| B^{0}\right\rangle & =\frac{\sqrt{3}}{2} A_{3 / 2}+\sqrt{\frac{1}{3}} A_{5 / 2} .
\end{aligned}
$$

Relating these amplitudes to the leading $b \rightarrow u \bar{u} d$ diagrams reveals that the tree level

diagrams contain only $\Delta I=\frac{3}{2}$ and $\Delta I=\frac{1}{2}$ transitions while the dominant gluonic penguin diagrams are purely $\Delta I=\frac{1}{2}$. The electroweak penguins which are not in weak phase with the strong penguins are generally assumed to be small [26] and therefore ignored. Eliminating $A_{5 / 2}$ from above, the transition amplitudes are found to satisfy the relation:

$$
A^{+-}+\sqrt{2} A^{00}=\sqrt{2} A^{+0} .
$$

Following the same procedure for the CP-conjugate processes leads to

$$
\bar{A}^{+-}+\sqrt{2} \bar{A}^{00}=\sqrt{2} \bar{A}^{+0} .
$$




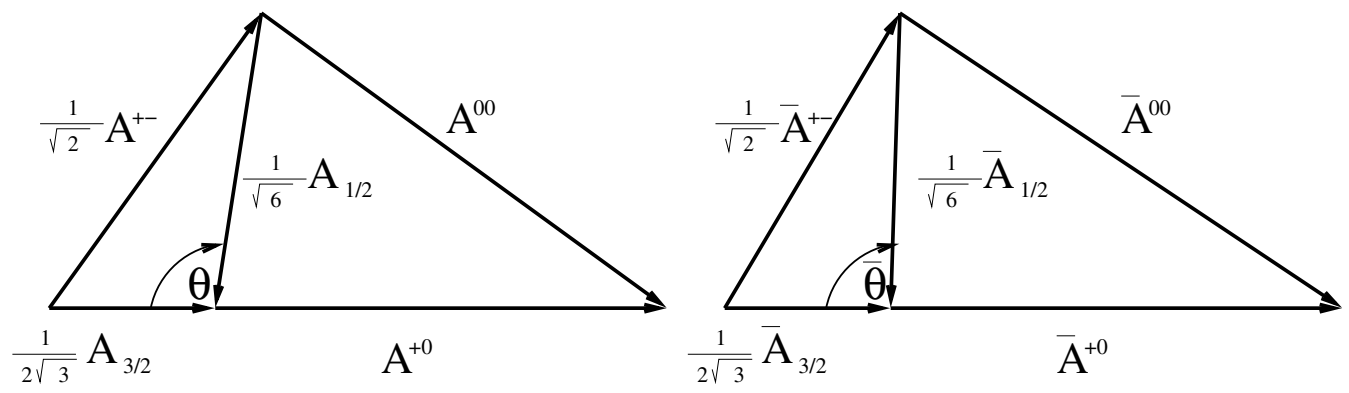

Figure 2.7: The $B \rightarrow \pi \pi$ isospin triangles.

Representing these two equations as triangles (see figure 2.7) illustrates graphically how these relations allow the extraction of $\left|A_{1 / 2}\right|,\left|A_{3 / 2}\right|,\left|\bar{A}_{1 / 2}\right|,\left|\bar{A}_{3 / 2}\right|$, and the phases $\theta$ and $\bar{\theta}$ from the six time integrated rates $B^{0} / \overline{B^{0}} \rightarrow \pi^{+} \pi^{-}, B^{ \pm} \rightarrow \pi^{ \pm} \pi^{0}$, and $B^{0} / \bar{B}^{0} \rightarrow \pi^{0} \pi^{0}$. However, these triangles could have been drawn upside-down, so an ambiguity exists in the determination of the angles $\theta$ and $\bar{\theta}$.

Since $A_{3 / 2}$ arises from the tree diagram only, it must carry the same phase. Therefore $\Lambda$ may be written as

$$
\Lambda_{\pi^{+} \pi^{-}}=\frac{q}{p} \frac{\bar{A}^{+-}}{A^{+-}}=-e^{2 i \alpha} \frac{\left|\bar{A}_{3 / 2}\right|-\frac{1}{\sqrt{2}}\left|\bar{A}_{1 / 2}\right| e^{i \bar{\theta}}}{\left|A_{3 / 2}\right|-\frac{1}{\sqrt{2}}\left|A_{1 / 2}\right| e^{i \theta}},
$$

allowing the extraction of $\sin 2 \alpha$ (with a 4 -fold ambiguity) from time-dependent measurements in $B^{0} \rightarrow \pi^{+} \pi^{-}$decays.

Though the effect of theoretical uncertainties due to assuming $S U(2)$ isospin symmetry and ignoring electroweak penguins is expected to be negligible for the extraction of $\alpha$, experimental difficulties inhibit the use of the isospin method in the near future. The color suppressed $\pi^{0} \pi^{0}$ decay is expected to have a small branching ratio and has not yet been observed [33]. This fact, coupled with the difficulties of reconstructing a high energy four photon final state and the need to tag the flavor of the $B$, indicates that the extraction of $\alpha$ through an isospin analysis is not in the immediate reach of 
the current experiments.

Bounds obtained by applying isospin symmetry argument are more likely to aid in testing of the CKM picture of CPV in the near future. Requiring closure of the isospin triangles in figure 2.7 leads to the relations [30]

$$
\begin{aligned}
& \cos 2\left(\alpha-\alpha_{\mathrm{Eff}}\right) \geq \frac{1-2 \mathcal{B}^{00} / \mathcal{B}^{+0}}{\sqrt{1-C_{\pi^{+} \pi^{-}}^{2}}}+\frac{\left(\mathcal{B}^{+-}-2 \mathcal{B}^{+0}+2 \mathcal{B}^{00}\right)^{2}}{4 \mathcal{B}^{+-} \mathcal{B}^{+0} \sqrt{1-C_{\pi^{+} \pi^{-}}^{2}}} \\
& \cos 2\left(\alpha-\alpha_{\mathrm{Eff}}\right) \geq \frac{1-4 \mathcal{B}^{00} / \mathcal{B}^{+0}}{\sqrt{1-C_{\pi^{+} \pi^{-}}^{2}}}+\frac{\left(\mathcal{B}^{+-}-2 \mathcal{B}^{+0}-2 \mathcal{B}^{00}\right)^{2}}{4 \mathcal{B}^{+-} \mathcal{B}^{+0} \sqrt{1-C_{\pi^{+} \pi^{-}}^{2}}}
\end{aligned}
$$

where $\mathcal{B}^{q q^{\prime}}=\frac{1}{2}\left(\left|A^{q q^{\prime}}\right|^{2}+\left|\bar{A}^{q q^{\prime}}\right|^{2}\right)$ are the CP averaged $B \rightarrow \pi^{(q)} \pi^{\left(q^{\prime}\right)}$ branching fractions. Setting $C_{\pi^{+} \pi^{-}}=0$ leads to the simpler but weaker Grossman-Quinn [29] bound

$$
\sin ^{2}\left(\alpha-\alpha_{\mathrm{Eff}}\right)<\frac{\mathcal{B}^{00}}{\mathcal{B}^{+0}}
$$

which permits the use of the upper limits on the $B^{0} \rightarrow \pi^{0} \pi^{0}$ branching fraction and measurements of the $B^{+} \rightarrow \pi^{+} \pi^{0}$ branching fraction to obtain the maximal deviation of the asymmetry measured in $B^{0} \rightarrow \pi^{+} \pi^{-}, \alpha_{\mathrm{Eff}}$, from the unitary triangle angle $\alpha$.

\subsection{2 $S U(3)$ Flavor Symmetry}

Invoking $S U(3)$ flavor symmetry, the penguin contributions to $B^{0} \rightarrow K^{+} \pi^{-}$and $B^{0} \rightarrow \pi^{+} \pi^{-}$are equal. This assumption leads to a relation which avoids the use of modes with $\pi^{0}$ 's [34]:

$$
\cos 2\left(\alpha-\alpha_{\mathrm{Eff}}\right) \geq \frac{1-2 \lambda^{2} \mathcal{B}_{K \pi}^{+-} / \mathcal{B}^{+-}}{\sqrt{1-C_{\pi^{+} \pi^{-}}^{2}}} .
$$

Here $\lambda$ is the Wolfenstein parameter. Unfortunately $S U(3)$ flavor symmetry breaking may cause up to a $30 \%$ uncertainty on the amplitudes [74]. 


\subsubsection{Calculations of Hadronic Decay Amplitudes}

Some earlier calculations of hadronic $B$ decay amplitudes assumed that the matrix element for $B \rightarrow h_{1} h_{2}$ factorizes:

$$
\left\langle h_{1} h_{2}\left|\mathcal{H}_{\mathrm{eff}}\right| B\right\rangle=\left\langle h_{1}\left|J_{1}\right| B\right\rangle\left\langle h_{2}\left|J_{2}\right| 0\right\rangle
$$

Predictions were then obtained by using the $B \rightarrow h_{1}$ form factor for the first term and decay constant of the $h_{2}$ meson for the second. This approach, now known as "naive factorization", ultimately proved to be incapable of accurately predicting certain branching fractions [36]. As a result non-factorizable contributions such as gluon exchanges between $h_{1}$ and $h_{2}$ appear important in many decays.

Formal approaches to isolating dominant non-factorizable contributions to $B^{0}=$ $\bar{b} d$ meson decay amplitudes exploit the relatively large mass of the $b$ quark to write an heavy quark effective field theory (HQFT) that describes the QCD interactions of a stationary $b$ quark with relativistic $d$ quark $[27,31,86]$. Under this approximation, the light quark decouples from the soft QCD interactions simplifying the calculation of the hadronic decay matrix elements. In the heavy quark limit $\left(m_{b}>>\Lambda_{Q C D}\right)$, calculations are performed using pertubation theory in $\alpha_{s}$. To leading power in $\Lambda_{Q C D} / m_{b}$ and $\alpha_{s}$ the results of naive factorization are reproduced. Though much progress has been made in past few years that have led to successful prediction of various measured branching fractions, the reliability of calculations based on this technique has not yet been fully established. Some debate still exists regarding the various corrections necessary to provide accurate predictions [15]. 


\section{Chapter 3}

\section{The Detector}

Modern accelerator-based high energy experiments are typically the product of large collaborations of the scientists and engineers who design, build, and maintain the machines that produce the exotic particles and the detectors that capture their existence. The data employed in this dissertation was collected by the BABAR detector which records the $e^{+} e^{-}$collisions inside the PEP-II storage ring at the Stanford Linear Accelerator Center (SLAC). The BABAR collaboration consists of nearly 600 physicists.

The machine's concept originated in 1987 with Piermaria Oddone's suggestion that an asymmetric electron-positron collider would provide an excellent environment for studying $\mathrm{CP}$ violation in $B$ decays at the $\Upsilon(4 s)$ resonance [37]. As we saw in the previous chapter, $B$ mesons had already been recognized as ideal probes of CPV and the SM. Two laboratories ultimately accepted the challenge of developing a program for $B$ meson CPV studies using an asymmetric high luminosity $e^{+} e^{-}$collider design: KEK in Japan and SLAC in California. At SLAC, the Positron Electron Project's (PEP) ring was upgraded to support a high energy electron beam and a new lower energy positron ring was installed. Four and half years after the start of construction, PEP-II's first collisions took place on July 23, 1998. Meanwhile the 
BABAR detector, after a proposal in 1994 and a detailed Technical Design Report in 1995, was assembled in the PEP-II's interaction region 2 (IR-2) hall. BABAR has been recording the PEP-II collisions since May 1999.

In order to achieve their physics goals, the machine, detector, software, and personnel were required to operate as a factory, producing, recording, and analyzing the decays of tens of millions of $B$ meson pairs a year. In this chapter we'll examine the PEP-II and BABAR's design basics. The next chapter will focus on the computing aspects of $B A B A R$ and the experimental inputs to the physics analyses. The majority of the information in these two chapters are derived from reference [38], which documents the BABAR hardware and software systems in detail.

\subsection{PEP-II}

In the previous chapter we demonstrated how the time-evolution of $B$ meson pairs coming from the $\Upsilon(4 s)$ (equation 2.23) are sensitive to $\mathrm{CPV}$ and provide a means of measuring CKM parameters. Experimentally producing an environment which permits such studies poses two challenges. First, when the $\Upsilon(4 s)$ is produced at rest, the small $B$ lifetime $\left(\tau_{B} \approx 1.5 \mathrm{ps}\right)$ and the small boost provided to mesons (the $\Upsilon(4 s)$ is only slightly more massive than the $B \bar{B}$ pair, so $\left.p_{B}^{*} \approx 340 \mathrm{MeV} / c^{2}\right)$ makes measurements of the separation of the two $B$ meson decay points an experimental impossibility given today's technologies. Second, the decays of B's to CP eigenstates are very infrequent, having branching fractions that are typically less than $10^{-4}$. These considerations necessitate that the collider designed for CPV studies in $B$ mesons should be energy asymmetric, producing $\Upsilon(4 s)$ with sufficient boost so that the decay points of the $B$ mesons are distinguishable, and high luminosity, yielding 
significant numbers of $B$ decays to $\mathrm{CP}$ eigenstates.

\subsubsection{Design}

SLAC's 2 mile linear accelerator feeds $9.0 \mathrm{GeV}$ electrons into a high energy ring (HER) and $3.1 \mathrm{GeV}$ positrons into a low energy ring (LER) which reside in the 2200 meter PEP-II circular tunnel. These particles, kept in orbit by magnets and radiofrequency $(\mathrm{RF})$ acceleration, are collided at one interaction region inside the BABAR detector. Here the beams, while being tuned with a series of quadrapole magnets, are brought together and then separated by a pair of dipole magnets. High luminosity is achieved by simultaneously maintaining several hundred bunches of electrons and positrons in each ring. The two ring design also aids in producing high luminosities by minimizing interactions between the beams.

With a center of mass energy at the peak of the $\Upsilon(4 s)$ resonance, about $10.58 \mathrm{GeV}$, the $e^{+} e^{-}$system is Lorentz boosted in the electron direction with $\beta \gamma \approx .55$. The resulting $B$ mesons travel an average of 260 microns along the electron beam before decaying. At this resonance, $e^{+} e^{-} \rightarrow B \bar{B}$ production accounts for nearly a quarter of the total hadronic cross-section (see table 3.1). The latest technical design and operating parameters of the PEP-II rings are listed in table 3.2.

\subsubsection{Operation and Performance}

Though PEP-II operates at the $\Upsilon(4 s)$ resonance, a small portion of the running time $(\approx 12 \%)$ is dedicated to data taking at a CM energy of $40 \mathrm{MeV}$ below the resonance in order to aid in background studies for analyses sensitive to $e^{+} e^{-} \rightarrow u \bar{u}, d \bar{d}, s \bar{s}, c \bar{c}$ events, known as continuum background.

Figure 3.1 displays the accumulation of total integrated luminosity from October 


\begin{tabular}{cc}
\hline \hline$e^{+} e^{-} \rightarrow$ & Cross-section/nb \\
\hline$B \bar{B}$ & 1.10 \\
$c \bar{c}$ & 1.30 \\
$s \bar{s}$ & 0.35 \\
$u \bar{u}$ & 1.39 \\
$d \bar{d}$ & 0.35 \\
\hline$\tau^{+} \tau^{-}$ & 0.94 \\
$\mu^{+} \mu^{-}$ & 1.16 \\
$e^{+} e^{-}$ & $\approx 40$ \\
\hline \hline
\end{tabular}

Table 3.1: Approximate production cross sections at PEP-II, including the experimental acceptance of $B A B A R$.

\begin{tabular}{lcc}
\hline \hline Parameters & Design & Best \\
\hline Energy HER/LER $(\mathrm{GeV})$ & $9.0 / 3.1$ & $9.0 / 3.1$ \\
Current HER/LER $(\mathrm{A})$ & $0.75 / 2.15$ & $1.050 / 1.775$ \\
Number of Bunches & 1658 & 800 \\
Bunch spacing $(\mathrm{ns})$ & 4.2 & $6.3-10.5$ \\
$\sigma_{x}(\mu \mathrm{m})$ & 110 & 120 \\
$\sigma_{y}(\mu \mathrm{m})$ & 3.3 & 5.6 \\
$\sigma_{z}(\mathrm{~mm})$ & 9 & 9 \\
Luminosity $\left(10^{33} \mathrm{~cm}^{-2} \mathrm{~s}^{-1}\right)$ & 3 & 4.602 \\
Luminosity $\left(\mathrm{pb}^{-1} /\right.$ day $)$ & 135 & 120 \\
\hline \hline
\end{tabular}

Table 3.2: PEP-II design and highest luminosity operating parameters. 


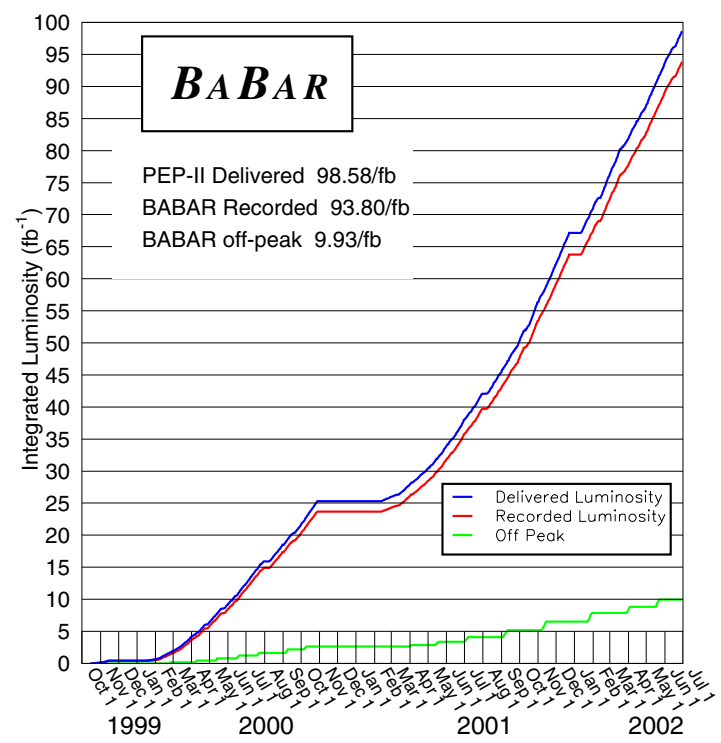

Figure 3.1: The integrated PEP-II luminosities delivered to and recorded by BABAR.

1999 to June 2002. The analysis in this dissertation employs $82 \mathrm{fb}^{-1}$ (or 88 million $B \bar{B}$ decays) of the on-resonance data. While the beam energies are calculated by PEP-II from the total magnetic bending strength and the average deviations of the $\mathrm{RF}$ accelerating frequencies from their mean values, the absolute luminosity, luminous region size, and beam position and angles are determined by $B A B A R$. The $B$ counting technique will be discussed in the next chapter.

\subsection{BABAR}

The BABAR detector was designed to meet the stringent requirements of an ambitious physics program. Given the small branching fractions and high physics backgrounds of many interesting $B$ decay modes and the sophisticated vertexing and flavor identification requirements of time-dependent $\mathrm{CP}$ analyses, the detector had to possess large and uniform acceptance, highly efficient and accurate charged and neutral par- 


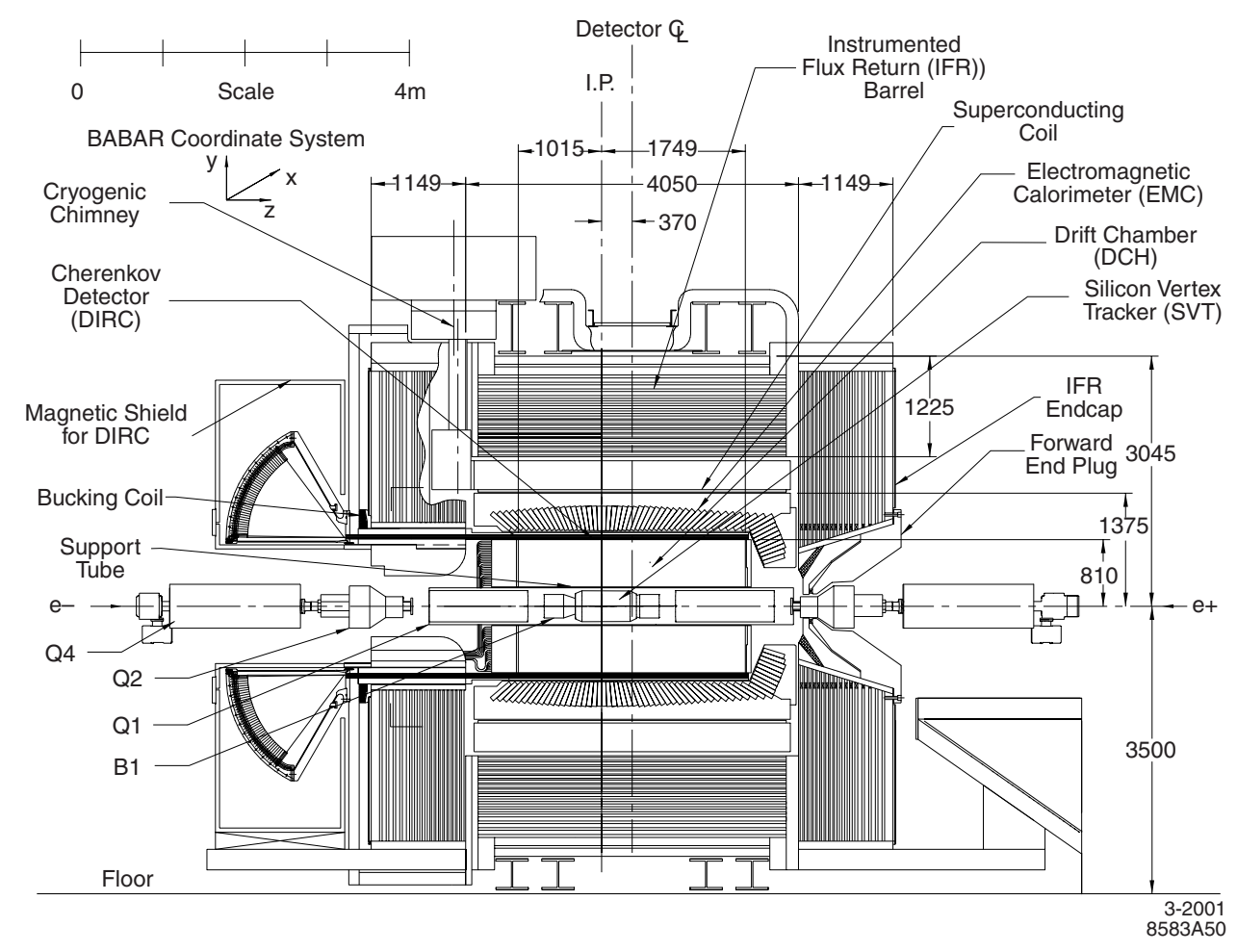

Figure 3.2: $y-z$ cross-section schematic of the BABAR Detector.

ticle reconstruction, and powerful particle identification, while operating reliably for long periods in a possibly high radiation environment. In this section we will briefly discuss various components of the BABAR detector.

Figures 3.2 and 3.3 display the $y-z$ and $x-y$ cross-section schematics of the $B A B A R$ detector, where the electron beam direction is defined as the $+z$ direction and $+y$ points upward. Five sub-detectors, built as nested concentric cylinders about the interaction point, are supported by an array of electronics that control, readout, and monitor every component. Inside a 1.5 Tesla super-conducting solenoid sits the tracking system, composed of the Silicon Vertex Tracker (SVT) and the Drift Chamber (DCH); the Detector of Internally Reflected Cherenkov light (DIRC) for particle identification; and the Electromagnetic Calorimeter (EMC). On the outside, 


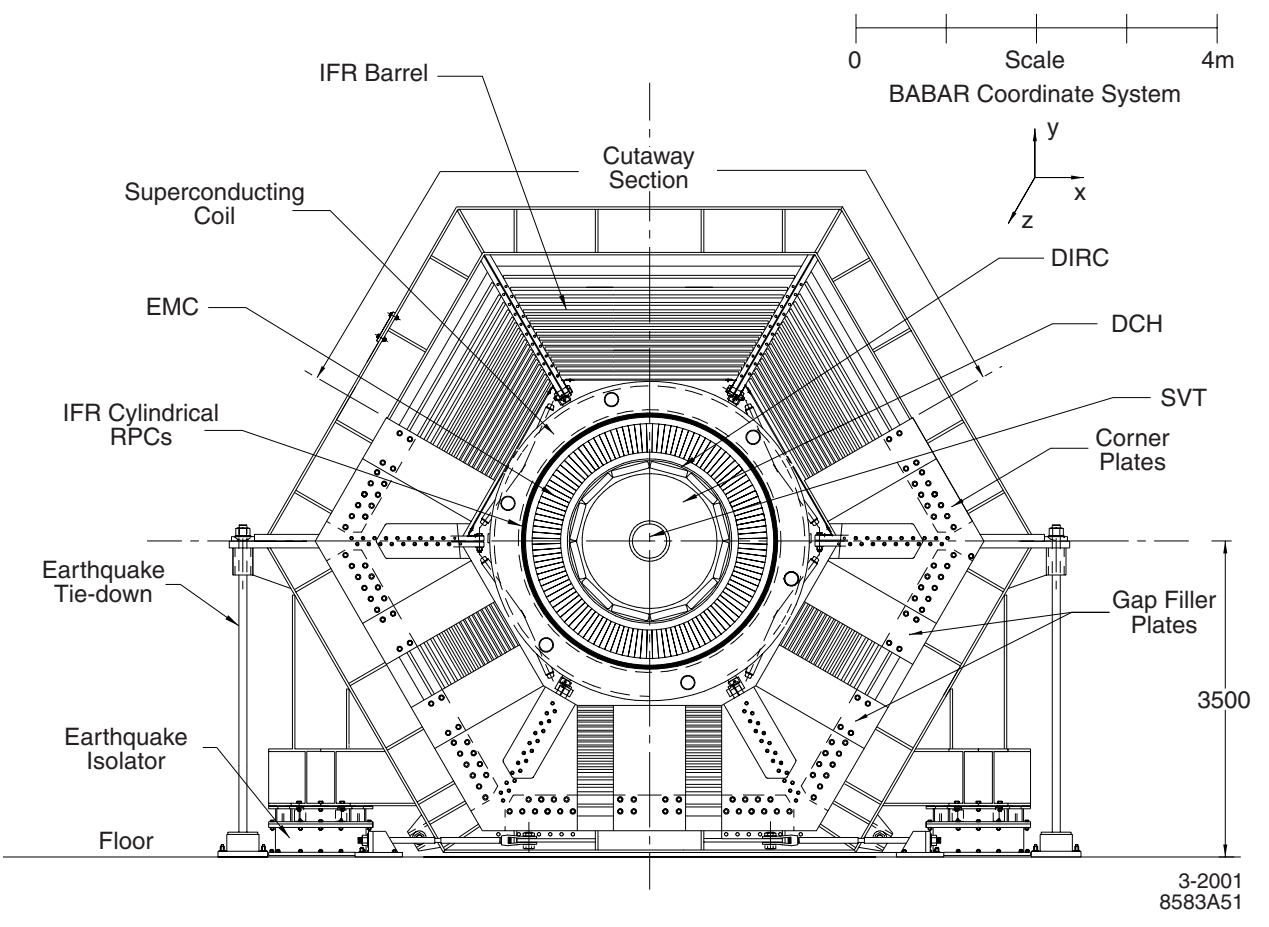

Figure 3.3: The $x-y$ cross-section schematic of the BABAR Detector. 
the steel Instrumented Flux Return (IFR) of the magnate provides muon identification and neutral hadron detection. Table 3.3 summerizes each detector's coverage and performance.

\subsubsection{Design considerations from PEP-II}

Sitting 3.5 meters above the floor of the experimental hall, the interaction region provided by PEP-II constrains the radius of BABAR's cylindrical design while the dipole and quadrapole magnets which steer and tune the beams into collision limit the forward/backward acceptance. The asymmetry of the machine is reflected in $B A B A R$ 's positioning with respect to the interaction point: an offset $37 \mathrm{~cm}$ in the direction of the LER maximizes the acceptance of the boosted system. Finally, in order to reduce perturbation by the tracking system solenoid, the detector axis is offset $20 \mathrm{mrad}$ relative to the beam axis in the horizontal plane.

The high luminosity environment provided by PEP-II produces various sources of large backgrounds and necessitates radiation hard detectors and electronics for $B A B A R$ to protect against damage, aging, and bandwidth limitations from extraneous signals. Though majority of the several $\mathrm{kW}$ of synchrotron radiation emanating from the quadrapole and dipole magnets is diverted by the design of the beam orbits, the vacuum-pipe aperture, and the masks, the bending of the beams into collision is still the primary source of machine backgrounds. Beam-gas backgrounds, caused by bremsstrahlung and Coulomb scattering off of residual gas molecules, are

enhanced after vacuum breeches. The subset of resulting energy-degraded particles which travel to the interaction region are typically bent by the dipoles, illuminating the extreme angles in the horizontal plane. Finally interactions of energy degraded electrons or positrons from radiative Bhabha scattering with various apertures pro- 
Table 3.3: Overview of the coverage, segmentation, and performance of the BABAR detector systems. The notation (C), (F), and (B) refers to the central barrel, forward and backward components of the system, respectively. The detector coverage in the laboratory frame is specified in terms of the polar angles $\theta_{1}$ (forward) and $\theta_{2}$ (backward). The number of readout channels is listed. Performance numbers are quoted for $1 \mathrm{GeV} / c$ particles, except where noted. The performances for the SVT and DCH are quoted for a combined Kalman fit (for the definition of the track parameters, see Section 4.2.1.)

\begin{tabular}{|c|c|c|c|c|c|}
\hline System & $\begin{array}{c}\theta_{1} \\
\left(\theta_{2}\right)\end{array}$ & $\begin{array}{c}\text { No. } \\
\text { Channels }\end{array}$ & $\begin{array}{c}\text { No. } \\
\text { Layers }\end{array}$ & Segmentation & Performance \\
\hline \multirow[t]{2}{*}{ SVT } & $20.1^{\circ}$ & $150 \mathrm{~K}$ & 5 & $50-100 \mu m r-\phi$ & $\sigma_{d_{0}}=55 \mu \mathrm{m}$ \\
\hline & $\left(-29.8^{\circ}\right)$ & $100-200 \mu \mathrm{m} \mathrm{z}$ & $\sigma_{z_{0}}=65 \mu \mathrm{m}$ & & \\
\hline \multirow[t]{4}{*}{$\mathrm{DCH}$} & $17.2^{\circ}$ & 7,104 & 40 & 6-8 mm & $\sigma_{\phi}=1 \mathrm{mrad}$ \\
\hline & $\left(-27.4^{\circ}\right)$ & & & drift distance & $\sigma_{\tan \lambda}=0.001$ \\
\hline & & & & & $\sigma_{p_{T}} / p_{T}=0.47 \%$ \\
\hline & & & & & $\sigma(d E / d x)=7.5 \%$ \\
\hline \multirow[t]{3}{*}{ DIRC } & $25.5^{\circ}$ & 10,752 & 1 & $35 \times 17 \mathrm{~mm}^{2}$ & $\sigma_{\theta_{C}}=2.5 \mathrm{mrad}$ \\
\hline & $\left(-38.6^{\circ}\right)$ & & & $(r \Delta \phi \times \Delta r)$ & per track \\
\hline & & & & 144 bars & \\
\hline \multirow[t]{2}{*}{$\operatorname{EMC}(\mathrm{C})$} & $27.1^{\circ}$ & $2 \times 5760$ & 1 & $47 \times 47 \mathrm{~mm}^{2}$ & $\sigma_{E} / E=3.0 \%$ \\
\hline & $\left(-39.2^{\circ}\right)$ & & & 5760 cystals & $\sigma_{\phi}=3.9 \mathrm{mrad}$ \\
\hline \multirow[t]{2}{*}{$\operatorname{EMC}(\mathrm{F})$} & $15.8^{\circ}$ & $2 \times 820$ & 1 & 820 crystals & $\sigma_{\theta}=3.9 \mathrm{mrad}$ \\
\hline & $\left(27.1^{\circ}\right)$ & & & & \\
\hline \multirow[t]{2}{*}{$\operatorname{IFR}(\mathrm{C})$} & $47^{\circ}$ & $22 \mathrm{~K}+2 \mathrm{~K}$ & $19+2$ & $20-38 \mathrm{~mm}$ & $90 \% \mu^{ \pm}$eff. \\
\hline & $\left(-57^{\circ}\right)$ & & & & $6-8 \% \pi^{ \pm}$mis-id \\
\hline \multirow[t]{2}{*}{$\operatorname{IFR}(\mathrm{F})$} & $20^{\circ}$ & $14.5 \mathrm{~K}$ & 18 & $28-38 \mathrm{~mm}$ & (loose selection, \\
\hline & $\left(47^{\circ}\right)$ & & & & $1.5-3.0 \mathrm{GeV} / c)$ \\
\hline \multirow[t]{2}{*}{$\operatorname{IFR}(\mathrm{B})$} & $-57^{\circ}$ & $14.5 \mathrm{~K}$ & 18 & $28-38 \mathrm{~mm}$ & \\
\hline & $\left(-26^{\circ}\right)$ & & & & \\
\hline
\end{tabular}


duce backgrounds that scale with the instantaneous luminosity. Comparisons of data taken with single HER and LER beam with colliding beam runs show that machine backgrounds constitute a significant portion of all triggers.

The BABAR detector was designed to record PEP-II's full luminosity for 10 years. In addition to radiation-hard components, a radiation protection system safe-guards the detector by determining radiation doses from diode leakage currents in the SVT and signals from PIN diodes mounted on small CsI(Tl) crystals in the DCH and EMC. At the same time DCH and IFR high voltage and DIRC and IFR counting rates are monitored to insure sub-detector safety. BABAR-initiated beam aborts are generally induced by instantaneous sources of radiation from dust particles trapped in the beam and non-Gaussian tails from beam-beam interactions.

\subsubsection{Physics Constraints}

$B A B A R$ was designed to provide reliable, high quality collection of $B$ decays through

- low-noise electronics and high bandwidth data-acquisition and detector control systems,

- detailed monitoring and automated calibration,

- large and uniform tracking acceptance, and

- excellent track reconstruction efficiency/resolution and photon energy/angular resolution.

However, the characteristics of two-body $B$ decays to charged kaons and pions presented some specific design requirement for BABAR. These decays are (we will revisit these issues in next chapter) 
- rare, with branching fractions of order $10^{-6}-10^{-5}$;

- plagued by high backgrounds from $e^{+} e^{-} \rightarrow$ light quark pairs;

- kinematically unique, carrying the highest momentum tracks of any $B$ decay;

- and difficult to distinguish from one another.

Most notably, the particle identification requirements of $B A B A R$ were heavily influenced by the high momentum pion/kaon discrimination demanded by the analysis of these modes. Also, in order to make time-dependent analyses feasible, BABAR needed good vertexing to resolve the $B$ decay points, and excellent lepton and kaon identification to identify the $B$ flavor. In the remaining sections of this chapter, we will describe the sub-detectors and sub-systems which permit BABAR to achieve its various design goals.

\subsubsection{Tracking}

Bent by the magnetic $1.5 \mathrm{~T}$ field of the super-conducting solenoid, the momenta of charge particles are determined from the curvature of their trajectories reconstructed from their interactions with the instrumented components of the SVT and DCH. The field, produced by currents in two layers of $3060 \mathrm{~mm}$ diameter, $3513 \mathrm{~mm}$ long niobium-titanium coils, is very uniform in the tracking volume, deviating at most by $2.5 \%$ in the direction transverse to the path of high momentum tracks.

The BABAR SVT (pictured in figures 3.4 and 3.5) provides excellent vertexing as well as low momentum charge particle reconstruction by providing stand-alone tracking capability with accurate position and impact parameter determination to the tracking system. Mounted on the beam pipe, this detector's $0.96 \mathrm{~m}^{2}$ of active 


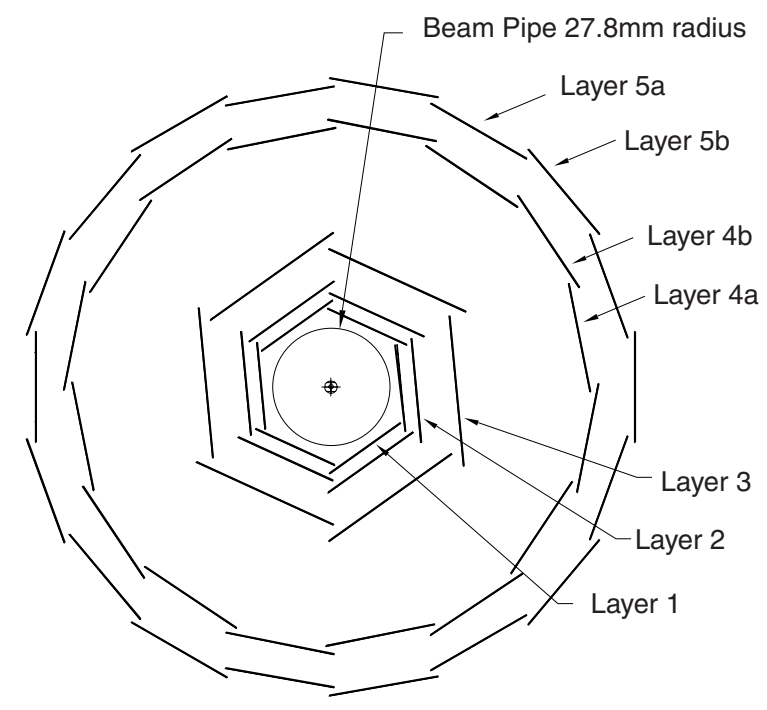

Figure 3.4: $x-y$ cross-section schematic of the BABAR's SVT Detector.

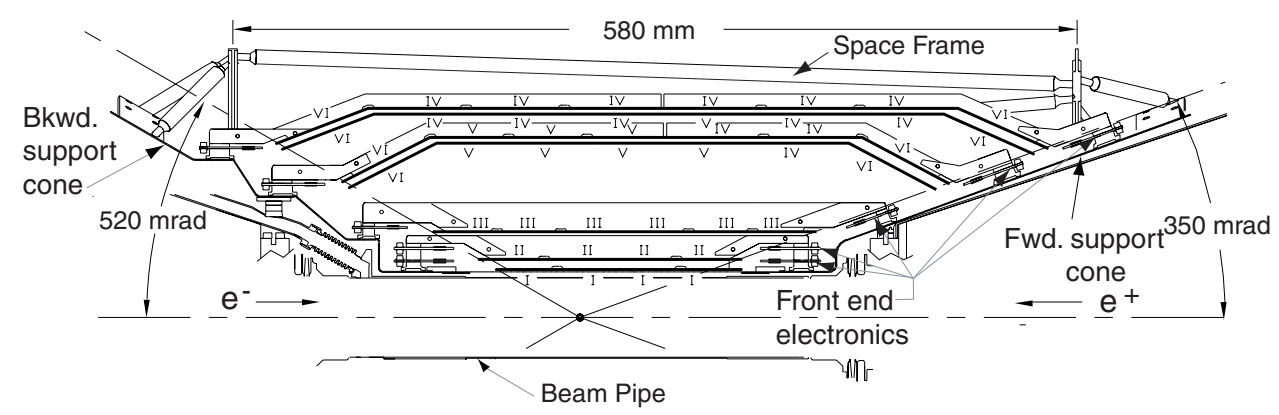

Figure 3.5: $y-z$ cross-section schematic of the BABAR's SVT Detector. 


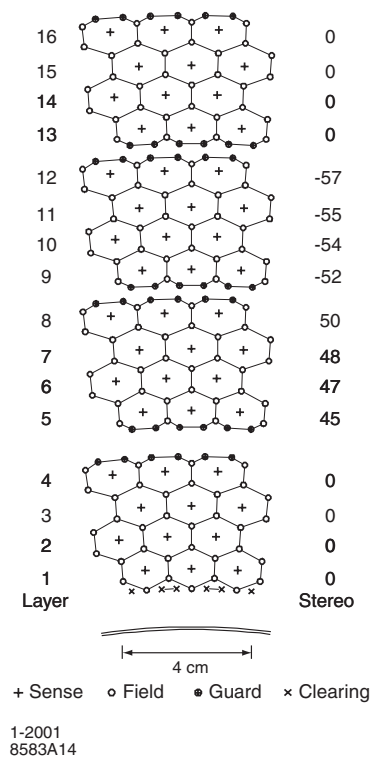

Figure 3.6: Schematic of the 4 inner layers of the BABAR DCH.

area is composed of five layers of 340 double-sided silicon wafers mounted on a carbonfiber frame. On the two faces of every wafer, strip sensors running orthogonal to ones on the opposite surface detect the passage of a charged particle, measuring both $z$ and $\phi \equiv \tan ^{-1}(x / y)$. The wafers are organized in half modules which read out at the two ends of the detector by fanout circuits to custom time-over-threshold (ToT) chips in a total of 150,000 channels. The inner three layers are placed close to the beam pipe, dominating the determination of track position and angles, while the outer two layers, which are arch-shaped to minimize the silicon use, are placed close to the DCH in order to aid in track matching with the DCH. Tilting of modules in the inner layers and dividing the outer layers into two sub-layers produces overlap regions which avoid gaps in the acceptance.

The DCH's reconstruction of track trajectories dominates BABAR's measurement of charge particle momenta. In this detector's $3 \mathrm{~m}$ long and $81 \mathrm{~cm}$ diameter cylin- 
drical volume, 7104 hexagonal cells of one grounded tungsten-rhenium sense wire surrounded by six aluminum field wires held at $>+1900 \mathrm{~V}^{1}$ detect traversing charge particles' ionization of a 80:20 mixture of helium:isobutane gas at 4 mbar above atmospheric pressure. This choice of wire and gas minimizes multiple Coulomb scattering, presenting less that $0.2 \%$ of the radiation length $\left(X_{0}\right)$ to tracks. The cells (see figure 3.6), grouped into 16 axial and 24 stereo layers with sense wires running parallel and at small angles to the beam axis, respectively, provide on average 40 spatial and ionization loss measurements for each track. The readout electronics, mounted on the backward end-plate in order to minimize the material in front of the forward calorimeter end-cap, measure the drift time, the integrated charge, and provide a single bit to the trigger. Section 4.2.1 describes the SVT and DCH track reconstruction and the resolutions of the measured track parameters.

\subsubsection{Charged Particle Identification}

High momentum particle identification is dominated by the DIRC's measurements of the cone angle of the Cherenkov light emitted as charge particles traverse 144 $4.9 \mathrm{~m} \times 1.7 \mathrm{~cm} \times 3.5 \mathrm{~cm}$ synthetic fused silica bars arranged into a 12-sided polygonal barrel which encircles the DCH cylinder. Taking advantage of the preservation of the Cherenkov angle through internal reflection within each bar, this detector images the ringed projections of the Cherenkov cones emitted out of the backward end of the bars on an array of photomultiplier tubes (PMT). These PMTs instrument the

\footnotetext{
${ }^{1}$ The $B A B A R$ data sample has been collected with the DCH at three different high voltages. Originally the wires were held at $1960 \mathrm{~V}$. After collecting $\approx 10 / f b$ of on-resonance data, the voltage was changed to $1900 \mathrm{~V}$ for another $\approx 10 / \mathrm{fb}$ in an effort to extend the lifetime of the chamber. Eventually $1930 \mathrm{~V}$ was chosen as optimal for both the chamber's longevity and detection efficiency.
} 


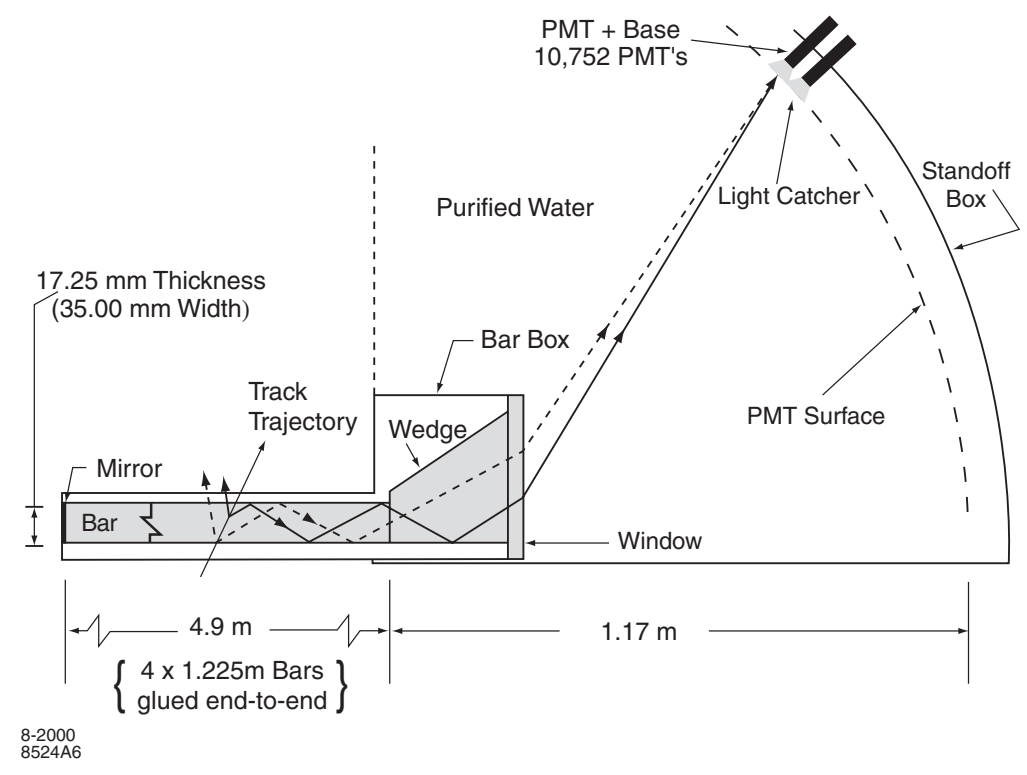

Figure 3.7: Schematic drawing illustrating the detection of Cherenkov photons by $B A B A R$ 's DIRC.

toroidal rear of a water-filled expansion region, known as the standoff box (see figure 3.7). The position and arrival time of the PMT signals permit the extraction of the Cherenkov angles when supplemented with track position and angle information. The timing information also assists in suppressing background photons. Covering $94 \%$ of the azimuth and $83 \%$ of the center of mass polar angle cosine, the DIRC provides Cherenkov angle measurements with a resolution of $\approx 3$ mrad for tracks with momenta starting at the Cherenkov threshold of $\approx 500 \mathrm{MeV}$ and separates pions/kaons up to $4 \mathrm{GeV}$. Details regarding the DIRC reconstruction and performance may be found in sections 4.2.2 and 5.1.3.

Measurements of energy loss $(d E / d x)$ by the tracking system permit charged particle identification at low momenta and allow separation of $<700 \mathrm{MeV}$ pions and kaons. In the SVT, $\approx 10$ ToT measurements are converted to $d E / d x$ using a 


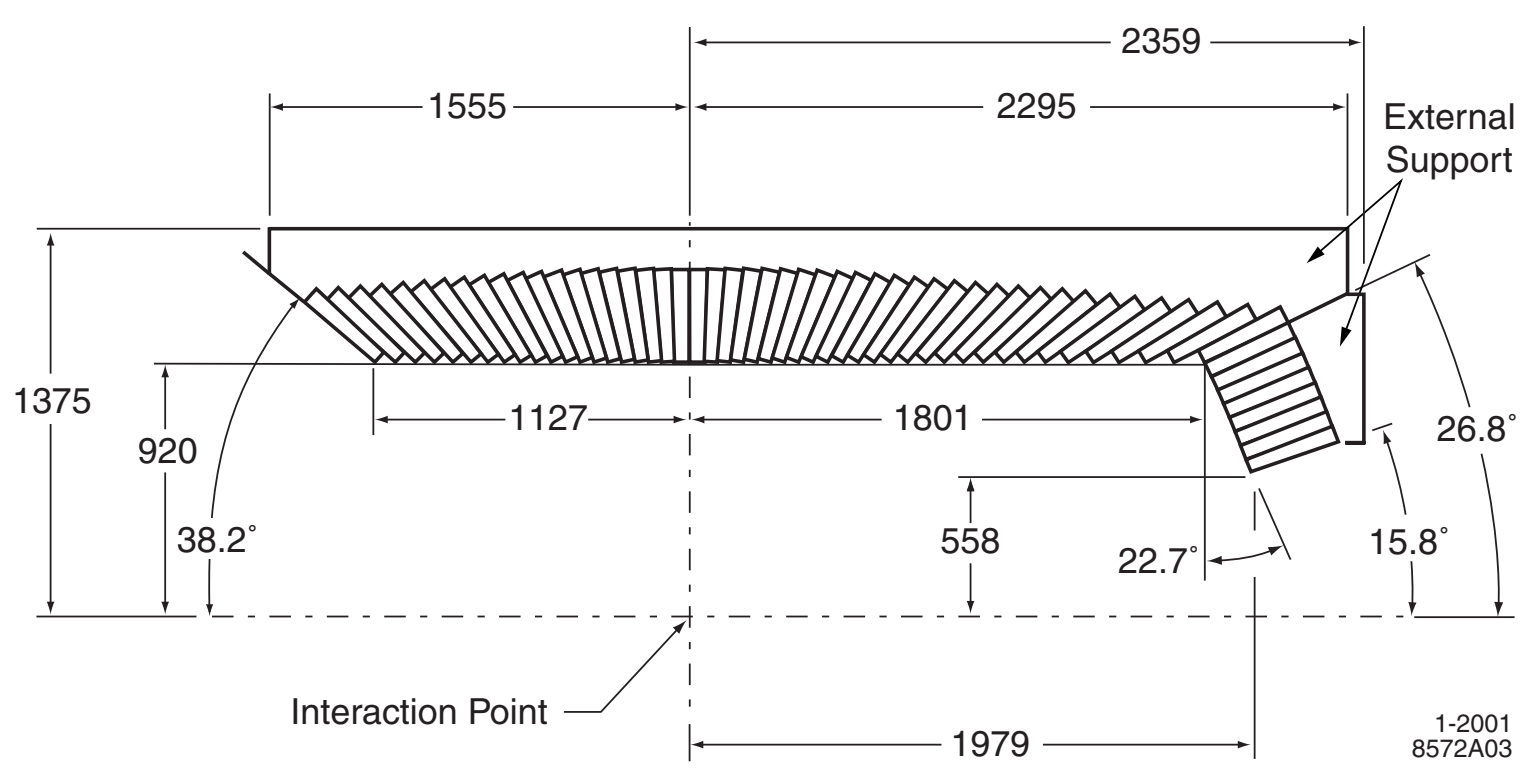

Figure 3.8: $y-z$ Schematic drawing of the top half of BABAR's EMC. The detector is axially symmetric around the $z$-axis. All dimensions are in $\mathrm{mm}$.

lookup table and a $60 \%$ truncated mean is calculated. In the DCH, an $80 \%$ truncated mean of the $\approx 40$ ionization loss measurements for each track provide a $7.5 \% \mathrm{dE} / \mathrm{dx}$ determination (see figure 4.7).

\subsubsection{Calorimetry}

The primary tasks of BABAR's EMC are the detection of photons, reconstruction of $\pi^{0}$ and $\eta$ decays, and the identification of electrons. This detector records the energy of the electromagnetic showers from photons and electrons in a finely segmented array of thallium-doped cesium iodide $(\mathrm{CsI}(\mathrm{Tl}))$ crystals (properties listed in in table 3.4). The crystals, with radiation lengths between 16.0 and $17.5 X_{0}$, are each instrumented with a pair of silicon photodiodes. The EMC, divided into two sections (a cylindrical barrel and a conical forward end-cap) which externally support each of the 6,580 crystals (see 


\begin{tabular}{cc}
\hline \hline Parameter & Value \\
\hline Radiation Length & $1.85 \mathrm{~cm}$ \\
Moliere Radius & $3.8 \mathrm{~cm}$ \\
Density & $4.53 \mathrm{~g} / \mathrm{cm}^{3}$ \\
Light Yield & $50,000 \mathrm{\gamma} / \mathrm{MeV}$ \\
\hline \hline
\end{tabular}

Table 3.4: Properties of $\mathrm{CsI}(\mathrm{Tl})$.

figure 3.8), determines the energy and direction of $90 \%$ of the photons emitted in the center of mass system. The desired resolution and reliability is achieved through low noise analog circuits and frequent and precise calibration of the electronics and energy response over the full dynamic range. Section 4.2.3 describes the EMC reconstruction and presents the energy and angular resolutions.

\subsubsection{Muon Identification}

806 Resistive Plate Chambers (RPC) placed inside the steel of the magnate flux return steel permit BABAR to discriminate muons from hadrons and to detect $K_{L}^{0} \mathrm{~s}$ and other neutral particles. The RPCs, arranged in 19 barrel and 18 end door layers and separated by steel of increasing thickness from $2 \mathrm{~cm}$ to $10 \mathrm{~cm}$, detect streamers from ionizing particles via external capacitive readout strips. In order to detect particles exiting the EMC, two additional layers with four readout plates are placed inside the magnet cryostat. The RPC strip segmentation provide measurements of track $\phi / z$ in the barrel and $x / y$ in the end-cap. 


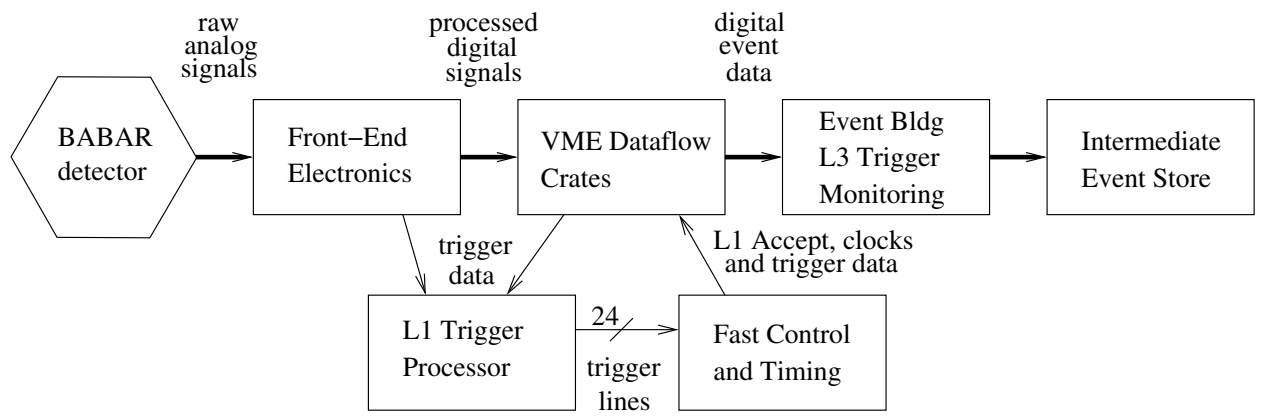

Figure 3.9: Schematic drawing the data path through BABAR's online system.

\subsubsection{The Online System}

$B A B A R$ 's electronics, trigger, data acquisition (DAQ), and online computing systems are composed of tightly coupled hardware and software. Maintainability and reliability is assured through data flow systems which are separate yet parallel to the monitor and control systems. Meanwhile standardized interfaces help manage complexity.

\section{Data Acquisition}

Figure 3.9 presents a schematic diagram of the DAQ system which transports data from the detector to mass storage. Designed for minimal dead-time, this system provides standardized interfaces for subsystem calibration, monitoring, and control of all data-flow.

For every detector subsystem, Front-End Electronics (FEE) perform signal processing, digitization, and downstream data transfer. The custom integrated circuits of these systems consist of amplifiers and digitizer, as well as buffers for trigger decision and transfer latencies. Since the FEEs are mounted on the detector in order to optimize performance and avoid noise pickup from long cables, they are generally inaccessible and required high levels of reliability. 


\begin{tabular}{crrr}
\hline \hline $\begin{array}{c}\text { Event } \\
\text { type }\end{array}$ & $\begin{array}{r}\text { Cross } \\
\text { section } \\
(\mathrm{nb})\end{array}$ & $\begin{array}{r}\text { Production } \\
\text { Rate }(\mathrm{Hz})\end{array}$ & $\begin{array}{r}\text { Level 1 } \\
\text { Trigger } \\
\text { Rate }(\mathrm{Hz})\end{array}$ \\
\hline$B \bar{B}$ & 1.1 & 3.2 & 3.2 \\
$u \bar{u}+d \bar{d}+c \bar{c}+s \bar{s}$ & 3.4 & 10.2 & 10.1 \\
$e^{+} e^{-}$ & $\sim 53$ & 159 & 156 \\
$\mu^{+} \mu^{-}$ & 1.2 & 3.5 & 3.1 \\
$\tau^{+} \tau^{-}$ & 0.9 & 2.8 & 2.4 \\
\hline \hline
\end{tabular}

Table 3.5: Cross sections, production and trigger rates for the principal physics processes at $10.58 \mathrm{GeV}$ for a luminosity of $3 \times 10^{33} \mathrm{~cm}^{-2} \mathrm{~s}^{-1}$. The $e^{+} e^{-}$cross section refers to events with either the $e^{+}, e^{-}$, or both inside the EMC detection volume.

A two-level hierarchical trigger system is the gate-keeper of the flow of data to the permanent storage. Configured to reduce beam background events rates, the Level 1 (L1) hardware trigger makes decisions based on number of charged tracks above a preset transverse momentum in the DCH, showers in the EMC, and tracks in the IFR. The three sub-system triggers are considered independently, maximizing redundancy in order to measure and monitor efficiencies. The L1 trigger has a maximum latency of $12 \mu$ s per beam crossing and is configured to produce an output of $\approx 1 \mathrm{kHz}$. Table 3.5 gives a break down of the principal physics processes contributing to this rate.

Online Data-flow (ODF), which provides data transport, buffering, and event building is implemented in a set of VME crates which house 157 Readout Modules (ROMs) running VxWorks. Configuration and readout of the FEEs are performed through 1.2 Gbits/s fiber links to the ROMs. These specialized VME-based processors handle Feature Extraction (FEX) of physics signals, perform gain and pedestal 
corrections, as well as data sparsification and formatting. The calibration data is stored in a dedicated conditions database. Using a Fast Control and Timing System (FCTS), events are built from data from the individual subsystems and shipped from the ROM modules to the Online Event Processing (OEP) workstations via $100 \mathrm{Mb}$ Ethernet. No dedicated counters are employed to associate events with beam crossings which occur at $4.2 \mathrm{~ns}$ intervals. Instead absolute timing is determined offline using DCH track segment timing, waveforms from EMC, and accelerator timing fiducials.

Running on a farm of Unix workstations, the OEP software collects and processes data from the ODF event builders, partially reconstructing the event in order to apply the Level 3 (L3) trigger algorithms and provide fast monitoring for the data taking personnel on shift. The L3 software examine the complete event information, categorizing and flagging physics, diagnostic, and calibration events for logging into 0.8 TB immediate storage. Typically, the L3 trigger is configured to deliver output rate of $\approx 120 \mathrm{~Hz}$, with an average event size of $\approx 28$ kbytes. The combined L1 and L3 efficiency for generic $B \bar{B}$ events is $>99.9 \%$.

Online Prompt Reconstruction (OPR) of the collected events occurs in as little as few hours after logging by farms of several hundred Unix workstations running in parallel. Using the raw detector signals and the partially reconstructed events of the L3 trigger, OPR performs full reconstruction of all physics events and select calibration events, applying preliminary analysis algorithms in order to categorize potentially interesting events before storing the results into an object database for further analysis. Monitoring and rolling calibration of reconstructed parameters is also applied at this stage, allowing examination of the data quality and propagation of time dependent constants into the conditions database for the processing of the next run. The following chapter will describe and assess the performance of some of 
these OPR algorithms.

\section{Detector Monitoring and Control}

Implemented in Experimental Physics Industrial Control System toolbox (EPICS), the Online Detector Control (ODC) system controls and monitors the electronics and environment of the detector and its support systems to assure safety. Monitoring of machine status, injection inhibition, and beam aborting is achieved through links to PEP-II systems. All collected data is archived into a browsable ambient database.

The online machinery is tied together by the Online Run Control (ORC) system whose logic manages the state of all systems and provides a user interface for calibrations and starting/stopping runs. Detector configurations are stored in a configurations database for reference during reconstruction. 


\section{Chapter 4}

\section{Analysis Computing}

The requirements of modern high energy physics experiments place strong demands on their supporting computing hardware and software systems. Recent discoveries of the field would have been improbable without the success of Moore's Law and the growth of computing budgets which continue to make current and future experiments feasible. Unburdened by the pressures of marketability yet challenged with the charge for scientific discovery, particle physics computing has often sat at the forefront of technology and hence engineered notable innovative developments.

From the onset BABAR computing strove to employ new technologies, arguing the technical and philosophical advantages of tools previously unused by high en-

ergy experiments [39]. With the migration from science's old standard programming language, FORTRAN, to the object oriented $\mathrm{C}++, B A B A R$ built a powerful software framework which now also serves other experiments. Meanwhile thousands of processors and hundreds of terabytes of storage provided the backbone. The resulting system reconstructed the volumes of raw data at nearly the same rate as it was collected, providing the complete dataset for timely analysis by the collaborators. 


\section{$4.1 \quad$ Overview}

The BABAR software framework provides an environment where software modules performing input/output, reconstruction, selection, and analysis tasks are sequentially executed while sharing standardized data objects through a common dictionary. Each module provides code to be called at the start, during, and at the end of the each job, run, or event. Reconstruction and analysis is performed by executables composed of a collection of these modules and configured and controlled at runtime by scripts written in TCL. These scripts define the sequence of module execution, build the data path by setting the input and output of the modules, and configure the behavior of each module.

Figure 4.1 displays a schematic of the path taken by the recorded data. Event reconstruction typically begins by reading data files containing the raw digitized output from the detector, the trigger classification, and the preliminary reconstruction of the L3 trigger. After filtering on the trigger output, interesting events are fed to sequences of subsystem reconstruction modules which extract and successively refine event time, tracking, calorimetry, and particle identification information. Eventually the individual subsystem information are associated and combined to create particle candidates.

At this stage, selection modules identify and tag physics and calibration events to be processed by different paths of module sequences for analysis and storage. In the physics path, selector modules identify well reconstructed charge and neutral particles and produce lists of candidates likely to be of a particular flavor. Combinatoric modules create composite candidates of $K_{S}^{0}, \pi^{0}$, and various $D$ mesons. This information is then considered by other selection modules provided by analysis groups which tag events of specific interest. 
The reconstruction algorithms are also applied to Monte Carlo simulated events, which are produced using JetSet [42] and a specialized decay generator package for $B$ decays called EvtGen [41]. A Geant4 based detailed simulation of the detector provides the detector response.

The reconstructed data is stored in federations of object databases built in Objectivity [40]. This system provides a means of storing and retrieving persistent copies of memory resident (or transient) $\mathrm{C}++$ objects. Physics and calibration events are stored in different streams (analogous to files) which may be independently accessed or retrieved from tape storage. Different depths of detail are stored in separate databases, named (in ascending order of size) tag, micro, mini, rec, and raw. Though all this information may be accessed for each event, only the tag and micro databases, which contain the highest level of reconstructed quantities, are maintained on disk. Events, which are grouped based on run number and tags acquired during reconstruction, are referenced by collections of pointers. Throughout the reconstruction, configuration and conditions information is retrieved and recorded into the various databases, permitting the propagation of rolling calibration constants from one run to the next. Periodically, the data written to the reconstruction production federations is moved to analysis federations which are accessible to all collaborators.

After full reconstruction, a skim executable regularly reads the analysis collections, determining updated tags of events and gathering them into smaller collections. At the same time the micro database is copied into a ROOT [43] based event store named KANGA for "Kind ANd Gentle Analysis" [44]. This alternative data format provides a portable storage solution which is generally used for analysis by the small computing facilities at various universities.

Typically, executables written by analysis groups scan the event collections by 


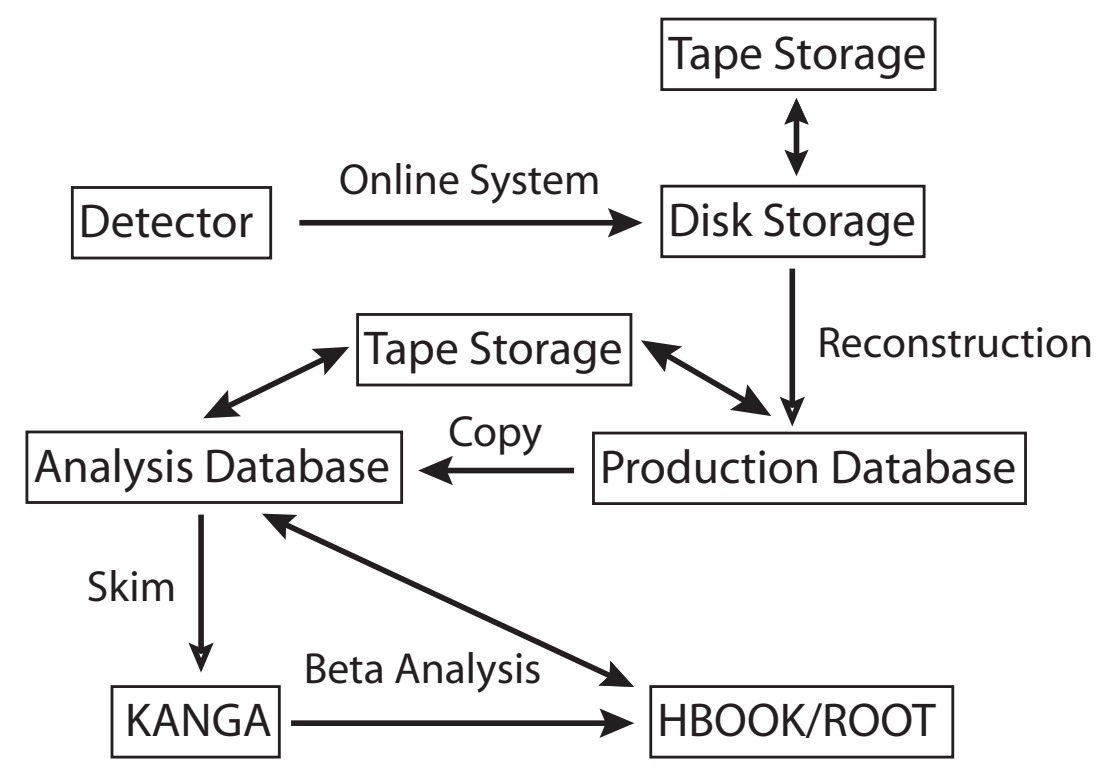

Figure 4.1: A schematic diagram of the propagation of the recorded data from the detector to HBOOK/ROOT where interactive analysis is performed.

examining the tag database and only read the micro database for interesting events. Detailed reconstruction of signals and more refined selections are then performed. The resulting candidates are either written back into the database for analysis by other executables, or outputted to HBOOK [45] or ROOT based ntuples which permit more interactive analysis.

\subsection{Event Reconstruction}

$B A B A R$ 's online prompt reconstruction (OPR) of events is performed on farms of a few hundred processors running a specialized version of the reconstruction executable. When processing a run, events are distributed among the processors, all of which simultaneously log their output to database servers. In this section we will briefly survey the reconstruction algorithms and discuss their performance. 


\subsubsection{Track Reconstruction}

The SVT and DCH's spatial recognition of the passage of a charge particle permits the extraction of the five helix parameters which describe the trajectories of tracks through the magnetic field of the solenoid. These parameters are the curvature $(\omega)$, the $x-y$ and $z$ distance of the point of closest approach (PoCA) to the $z$-axis $\left(d_{0}\right.$ and $\left.z_{0}\right)$, the track azimuth angle $\left(\phi_{0}\right)$ at the PoCA, and the dip angle of the track from

the transverse plane $\tan (\lambda)$. Searching for charged tracks begins in the L3 trigger's DCH pattern recognition and track finding based on the L1 trigger's track segment finder module algorithms which search for four contiguous track segments (known as a hit) in two dimensions. The L3 tracks built from these segments provide the first estimate of $d_{0}, \phi_{0}$, and the event starting time $t_{0}$, and provide the seeds for the off-line tracking algorithms.

In the offline reconstruction, drift time-to-distance relations, extracted from $e^{+} e^{-}$ and $\mu^{+} \mu^{-}$events, provide typical position resolution between 0.1 and $0.4 \mathrm{~mm}$ in each cell (see figure 4.2). Here, three track finders and fitters, based on the Kalman filter algorithm [46], reconstruct trajectories in three dimensions, employing detailed maps of the detector material and the magnetic field to account for energy loss and field variations. The first track finder starts with the L3 tracks and then search for additional hits. Removing the hits associated with these tracks from the event produces a cleaner environment for determining a $t_{0}$ and for the reconstruction of particles that either did not pass through the entire DCH or did not originate from the interaction point by the two other track finders.

The SVT provides $z$ and $\phi$ measurements with typical resolution between 15 and $40 \mu m$ (see figure 4.3). The DCH tracks are extrapolated through the intervening material into the SVT, where additional segments from the SVT are added. A combined 


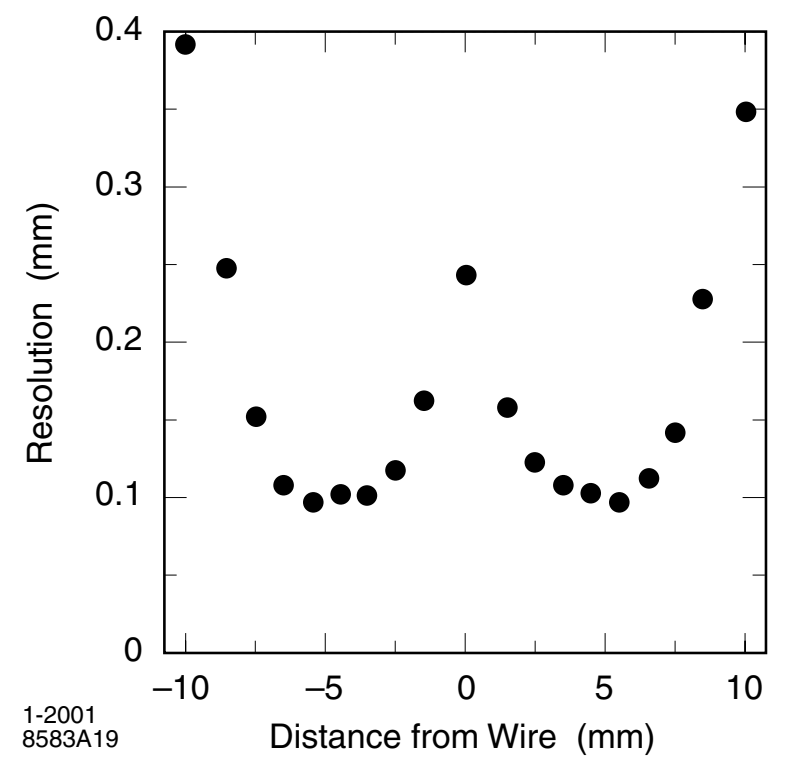

Figure 4.2: DCH position resolution as function of the drift distance in layer 18, for tracks on the left and right side of the sense wire, obtained from fits to track residual distributions in multi-hadron events. The data are averaged over all cells in the layer.
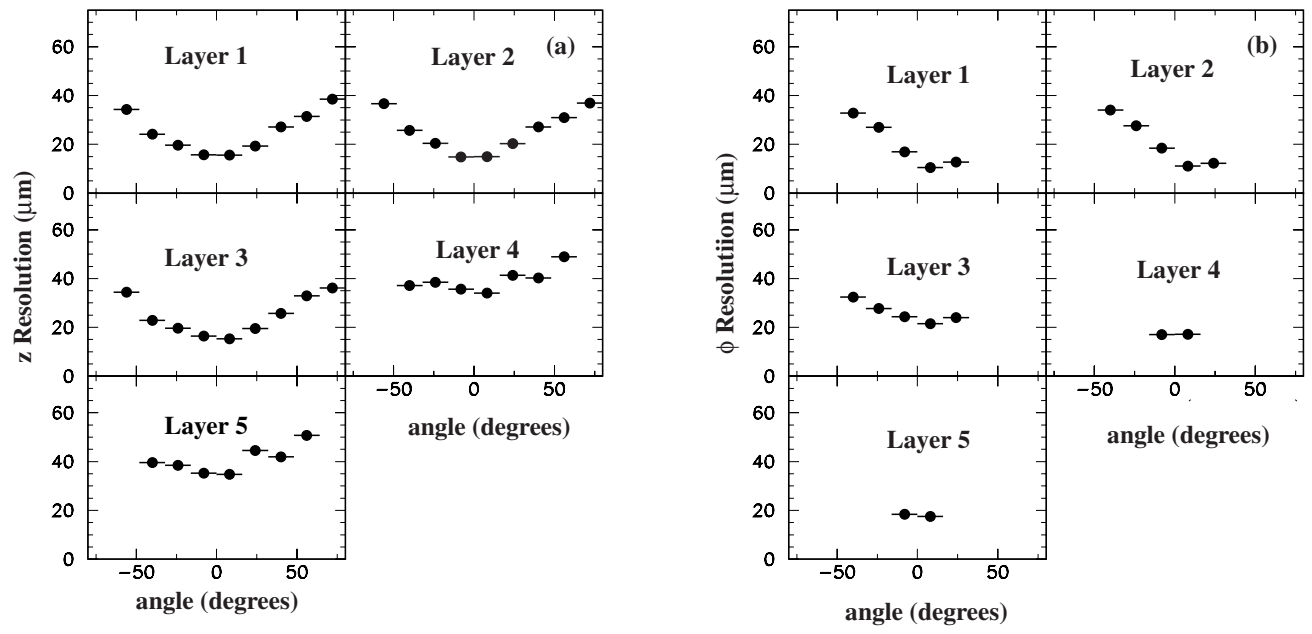

Figure 4.3: SVT hit resolution in the (a) $z$ and (b) $\phi$ coordinate in microns, plotted as a function of track incident angle in degrees. 


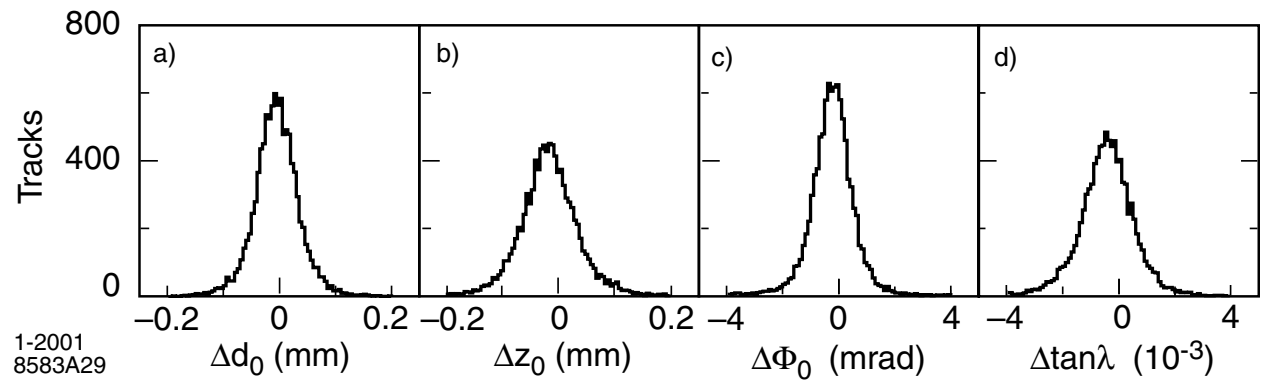

Figure 4.4: The differences (a) $\delta d_{0}$, (b) $\delta z_{0}$, (c) $\delta \phi_{0}$, and (d) $\delta \tan \lambda$ between fitted track parameters of the two halves of cosmic ray muons with $p_{t}>3 \mathrm{GeV}$.

SVT and DCH Kalman fit produces the final parameters for the resulting tracks. Any remaining SVT hits are examined by two SVT only track finders. The first builds tracks from at least four matched $\phi$ and $z$ hits (known as space points), while the second forms helices from $\phi$ hit circle trajectories by adding $z$ hits. A final algorithm attempts to merge tracks found by only one of the two tracking systems.

Track parameter resolutions have been assessed in cosmic ray events by comparing fits to cosmic track halves before and after the interaction region. Figure 4.4 displays parameter difference distributions for the two tracks when their transverse momentum is $>3 \mathrm{GeV}$. These plots infer resolutions of $\sigma_{d_{0}}=23 \mu \mathrm{m}, \sigma_{\phi_{0}}=0.43 \mathrm{mrad}, \sigma_{z_{0}}=29 \mu \mathrm{m}$, and $\sigma_{\tan \lambda}=0.53 \cdot 10^{-3}$. A similar comparison in figure 4.5 provides the $p_{t}$ dependence of the $p_{t}$ resolution, which is parameterized by $\sigma_{p_{t}}=(0.13 \pm 0.01) \% \cdot p_{t}+(0.45 \pm 0.03) \%$.

Since the SVT and DCH can independently track particles, the absolute DCH tracking efficiency is determined as the ratio of number of tracks reconstructed by the $\mathrm{DCH}$ to number of tracks detected by the SVT which project into the DCH volume. This procedure is applied to both data and Monte Carlo events in order to measure the ratio of the true tracking efficiencies to the expected values from the simulation. This ratio is then applied as a correction factor when calculating the tracking acceptance 


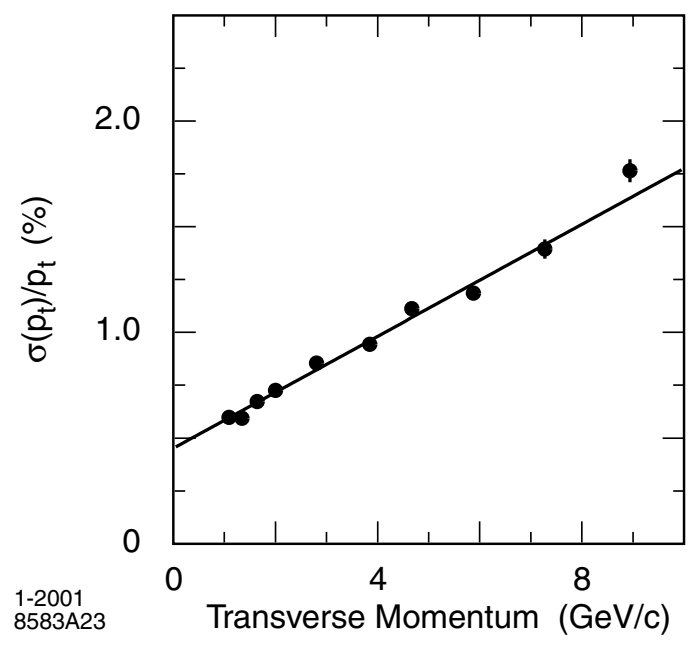

Figure 4.5: The transverse momentum resolution determined from cosmic ray muons. and reconstruction efficiencies for any decay mode from the Monte Carlo simulation. For each DCH high voltage period (see footnote in section 3.2.3), the efficiencies are calculated for good quality tracks, defined to satisfy the requirements:

- $p<10 \mathrm{GeV}$,

- $d_{0}<1.5 \mathrm{~cm}$,

- $\left|z_{0}\right|<10 \mathrm{~cm}$,

- $p_{T}>100 \mathrm{MeV}$, and

- 12 or more DCH hits,

in bins of track $p_{T}, \theta, \phi$, and number of tracks (satisfying the first 3 requirements) in the event. The results, which indicate that the overall tracking efficiency is above 95\%, are displayed in figure 4.6 for runs taken with DCH high voltage of $1930 \mathrm{~V}$, which represent most of the data available. The systematic uncertainty for determining the tracking efficiency for $B$ decays is estimated to be $1.3 \%$ per track in the decay. 

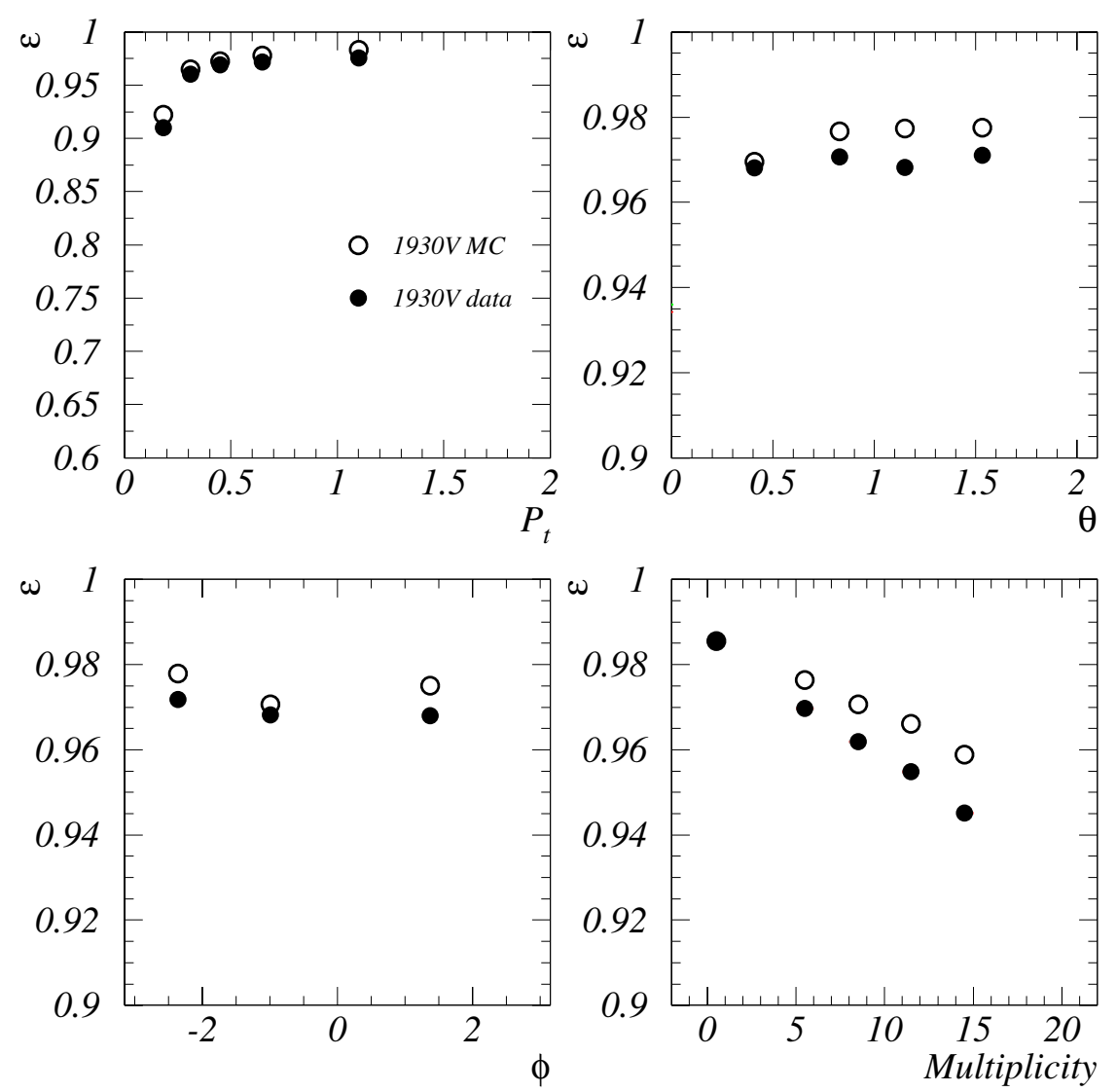

Figure 4.6: Data and Monte Carlo absolute tracking efficiency of good tracks in bins of track $p_{T}, \theta, \phi$, and number of tracks in the event, measured in runs with DCH high voltage of $1930 \mathrm{~V}$. 


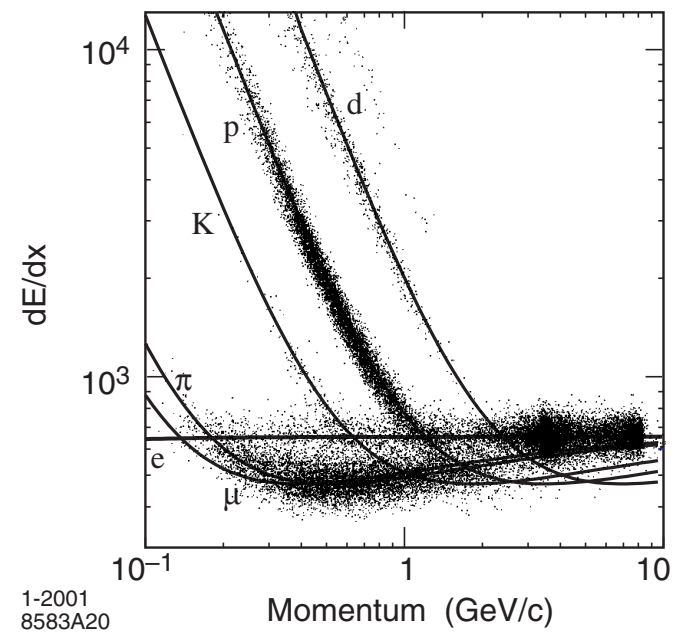

Figure 4.7: $\mathrm{DCH} d E / d x$ measurements as a function of track momenta. The solid curves represent the Bethe-Bloch expectations for various mass hypotheses.

\subsubsection{Particle Identification}

Below the Cherenkov threshold of the DIRC, the DCH $d E / d x$ measurements dominate $B A B A R$ 's particle identification of tracks. The DCH FEX algorithms extract the charge collected per single cell. For each track, a $80 \%$ truncated mean of $\approx 40$ such measurements, corrected for gas pressure and temperature variations, cell geometry, signal saturation, non-linearities at large dip angles, and cell entrance angle, provides a $7.5 \%$ resolution on $d E / d x$. Figure 4.7 displays the momentum dependence of this measurement in a sample consisting of particles with various masses.

The DIRC's measurement of the Cherenkov cone angle $\theta_{c}$ is BABAR's primary tool for identifying high momentum tracks. The reconstruction algorithm associates PMT signals with tracks, extracting a $\theta_{c}$ measurement when sufficient photons are available for a fit. Starting from the entrance angle of a track into a particular fused silica bar, the emission angle and arrival time of possible Cherenkov photons is reconstructed 

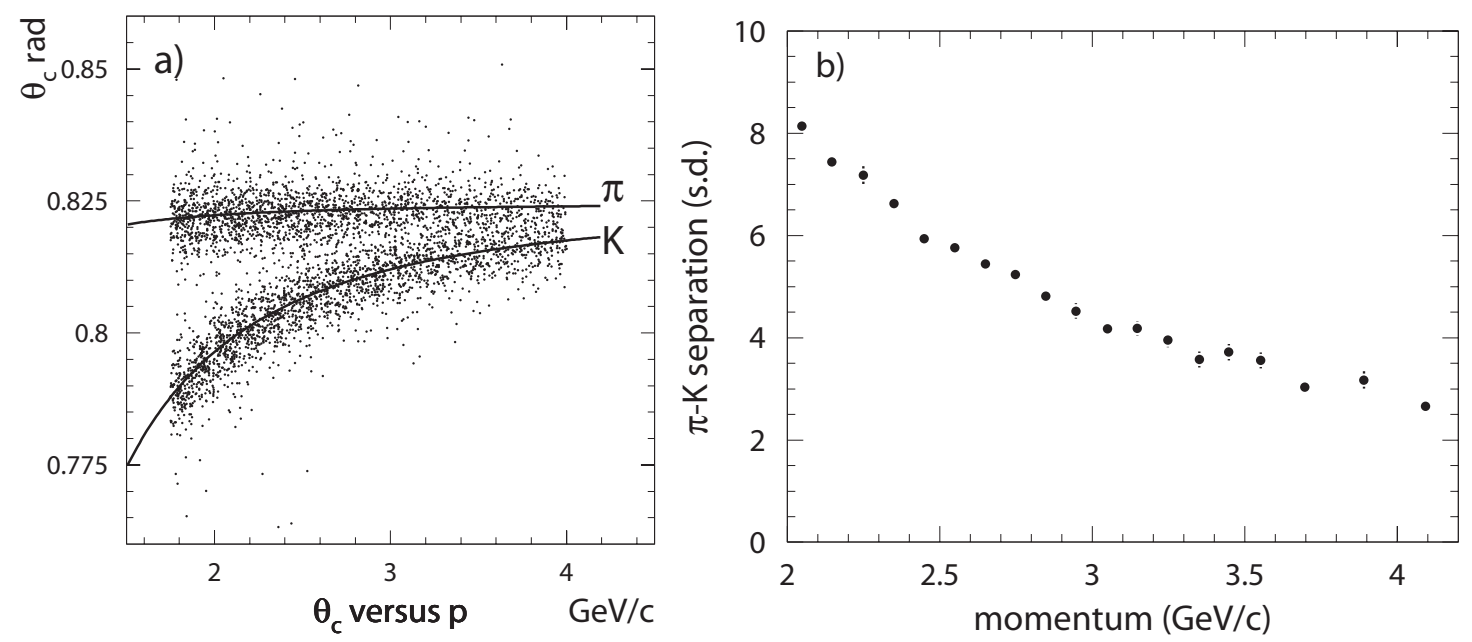

Figure 4.8: (a) The $\theta_{c}$ of kaons and pions versus the track momenta, and (b) the separation in standard deviations between pions and kaons as function of momentum, from the control sample described in section 5.1.3.

from the space-time coordinates of candidate PMT signals, providing a measurement of each photon's $\theta_{c}$ and $\phi_{c}$ (the azimuth angle of the Cherenkov photon around the track direction) with a 16-fold ambiguity. Timing and geometrical considerations typically reduce the ambiguities to 3 and the background by a factor of 40 . Finally, a maximum likelihood fit to the photons associated to each track extracts its $\theta_{c}$ and number of signal $\left(N_{\gamma}\right)$ and background photons. The resulting $\theta_{c}$ resolution scales as $1 / \sqrt{N_{\gamma}}$, where $N_{\gamma}$ is around 20 for short track path lengths in the radiator, typically at small polar angles, and 65 for the longer path lengths at the extreme polar angles. Section 5.1.3 discusses a technique for assessing the $\theta_{c}$ resolution. The average $\theta_{c}$ resolution is $\approx 3 \mathrm{mrad}$, which provides pion/kaon separation of $>2.2 \sigma$ at $4 \mathrm{GeV}$. Figure 4.8 plots the $\theta_{c}$ versus momentum profile and the measured standard deviations of separation between pions and kaons over the momentum range of tracks from two-body $B$ decays. 


\subsubsection{Calorimetry}

The electromagnetic shower produced by a charged or neutral particle in the EMC forms a cluster of energy deposits spread over many adjacent crystals. Meanwhile, photons from high momentum $\pi^{0} \rightarrow \gamma \gamma$ decays often illuminate adjacent crystals, producing two energy maxima (known as bumps) within one cluster. The EMC reconstruction algorithm searches for seed crystals which register an energy deposit of $E>10 \mathrm{MeV}$, and then builds a cluster by adding crystals with $E>1 \mathrm{MeV}$ which are either adjacent to another $E>3 \mathrm{MeV}$ crystal in the cluster or the seed. Crystals with energy $E$ satisfying $E^{\prime} / E<0.5(N-2.5)$, where $E^{\prime}$ is the highest energy of the neighboring $N$ crystals with $>2 \mathrm{MeV}$, are identified as constituting a local maxima. Bumps are built from these crystals with an energy determined by an algorithm which iterates the fraction of energy contributed by each crystal in the cluster until the bump centroid is stable to a tolerance of $1 \mathrm{~mm}$. Another center-of-gravity algorithm locates the bump position using logarithmic crystal weights. A cluster association with a charged particle is made if the projection from the bump centroid to the inner face of the calorimeter is consistent with a track trajectory. Otherwise, the bump is considered a neutral particle with a trajectory originating at the interaction point. Good clusters are defined as possessing energy $E>30 \mathrm{MeV} / c$, lateral shape parameter $\lambda_{\mathrm{LAT}}<1.1$ [47], and lab frame polar angle $0.41<\theta_{\mathrm{LAB}}<2.409$ (i.e. within the fiducial volume of the EMC).

The photon energy resolution is measured from a radioactive source (at the low end), $e^{+} e^{-}$Bhabha scattering events (at the high end), and decays of $\chi_{c 1}, \pi^{0}, \eta$, and other particles (in between). A fit over this data provides the energy dependence of 

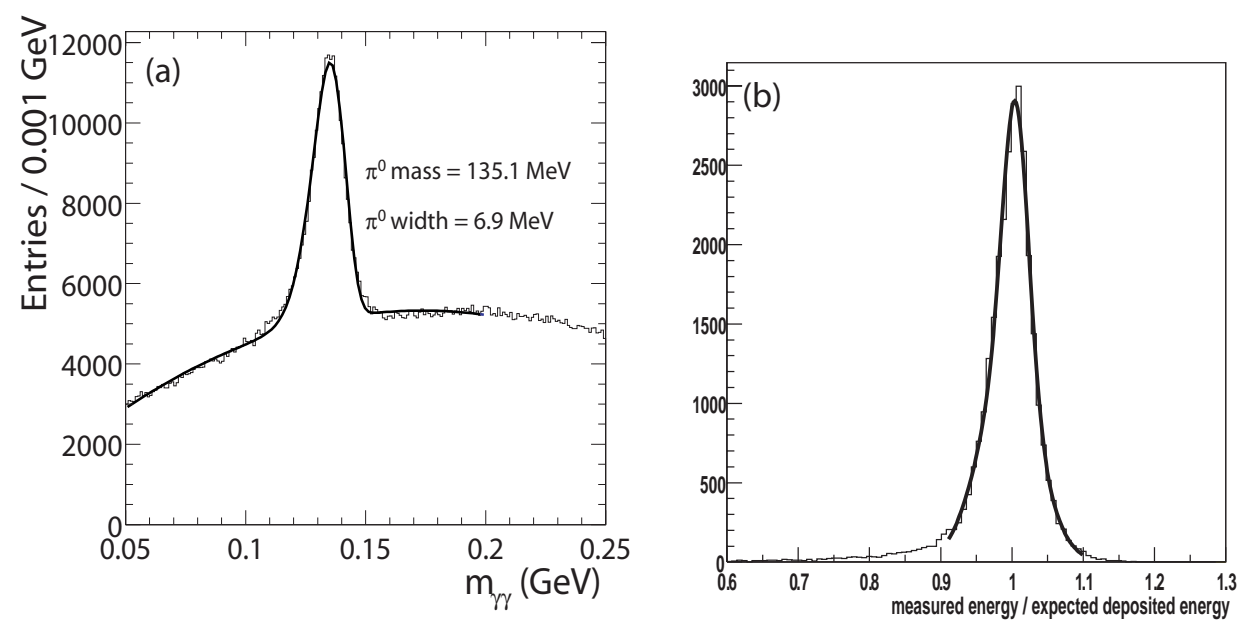

Figure 4.9: (a) The $\pi^{0}$ mass distribution reconstructed from two photon candidates in hadronic events overlaid with a fit to the data. (b) The ratio of measured to expected energy for electrons in Bhabha events overlaid with a Gaussian fit. The expected value is calculated from the production angle. The resolution is $1.9 \%$.

the resolution:

$$
\frac{\sigma_{E}}{E}=\frac{(2.32 \pm 0.30) \%}{\sqrt[4]{E(\mathrm{GeV})}}+(1.85 \pm 0.12) \% .
$$

Similarly studies of $\pi^{0}$ and $\eta$ decays to two photons of approximately equal energy provide an empirical parameterization of energy dependence of the angular resolution:

$$
\sigma_{\theta}=\sigma_{\phi}=\frac{(3.87 \pm 0.07)}{\sqrt{E(\mathrm{GeV})}}+(0.00 \pm 0.04) \mathrm{mrad}
$$

Typical $\pi^{0}$ mass resolution in hadronic events is $6.9 \mathrm{MeV} / c^{2}$ (see figure 4.9a). Figure $4.9 \mathrm{~b}$ displays the measured over expected energy ratio for Bhabha events. Figure 4.10 shows the ratio of deposited energy over the measured momentum for a sample of electrons. 


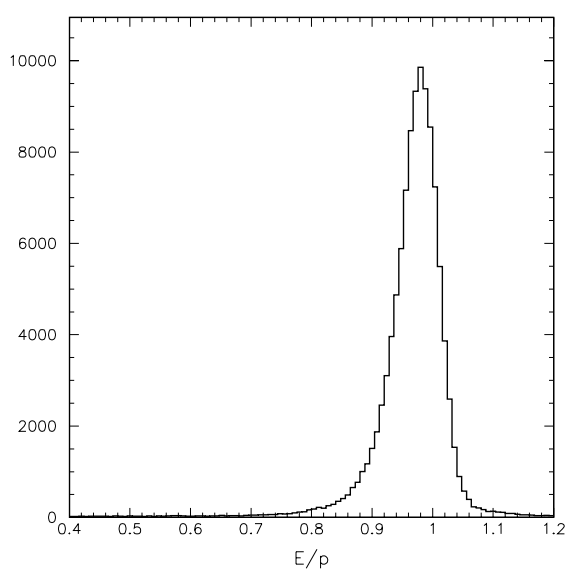

Figure 4.10: The ratio $E / p$ of deposited energy in the EMC over the measured momentum by the tracking system for electrons from $\gamma \gamma \rightarrow$ eeee events.

\subsubsection{Muon Identification}

The IFR reconstruction begins with the grouping of hits in the RPCs into clusters. One-dimensional IFR clusters are formed from groups of adjacent hits in one of the two readout coordinates. Adjacent one-dimensional clusters (of the same coordinate) in different layers are then combined to create two-dimensional clusters. Finally, threedimensional clusters are created in each sector from combinations of two-dimensional clusters in different coordinates with less than three layers missing in one of the two coordinates. A charged particle is associated to IFR cluster(s) if the cluster is a predefined distance from the intersection of its trajectory with the RPC planes, computed by extrapolating the track into the IFR and accounting for the non-uniform magnetic field, multiple scattering, and average energy loss. The identification of muons is based on

- comparison of the total number of interaction length traversed with the expec- 
tation for a muon,

- the average number and RMS of the distribution of RPC strips per layer,

- the $\chi^{2}$ of the geometric match between the projected track and the centroids of its associated clusters in each layer, and

- the polynomial fit to the two-dimensional IFR clusters.

The performance of the muon identification is assessed on kinematically selected sam-

ples of muons from $e^{+} e^{-} \rightarrow \mu \mu e e, \mu \mu \gamma$ final states and pions from three-prong $\tau$ and $K_{s} \rightarrow \pi^{+} \pi^{-}$decays. Figure 4.11 displays the muon detection efficiency and pion misidentification probability for a selection which achieves nearly $60 \%$ efficiency with a pion fake rate of $<2.5 \%$. The BABAR IFR has experienced a loss in muon detection efficiency of approximately 1\% per month. Figure 4.12 illustrates the observed effect.

\subsection{Analysis Software}

A standardized set of $\mathrm{C}++$ classes define the basic building blocks of the analysis machinery of the BABAR software. This library, known as Beta, provides the objects which represent the particle candidates as well as common operations and variables. Analysis executables manipulate lists of these candidates with framework modules implementing general purpose tasks such as selection, filtering, combinatorics, and ntuple output. All analyses begin by identifying, reconstructing, and outputting interesting events in a sequence of these modules. Widely used sets of well maintained libraries of such modules provide consistency, reliability, and standardization to BABAR's numerous physics analyses. These include publicly maintained and optimized particle identification selectors and combinatoric engines with efficiency and 


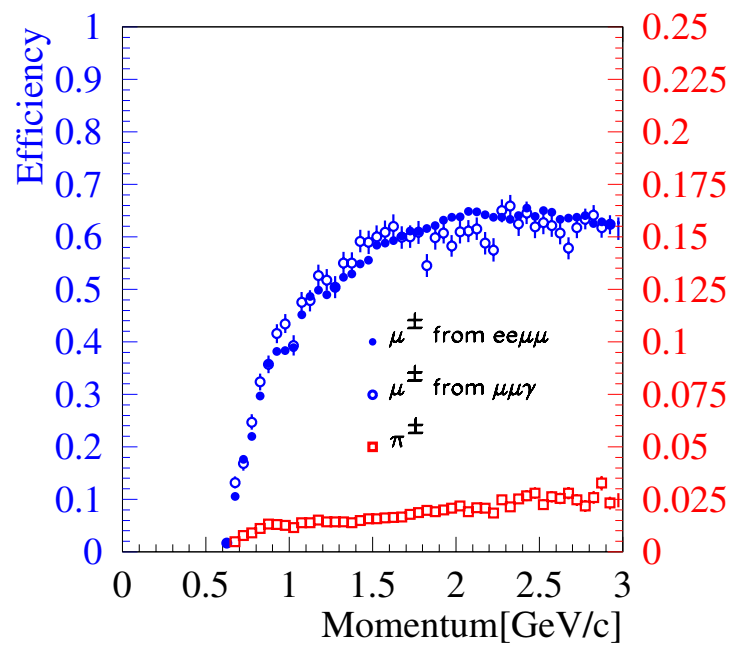

Figure 4.11: Muon efficiency (left scale) and pion misidentification probability (right scale) as a function of the track momentum.

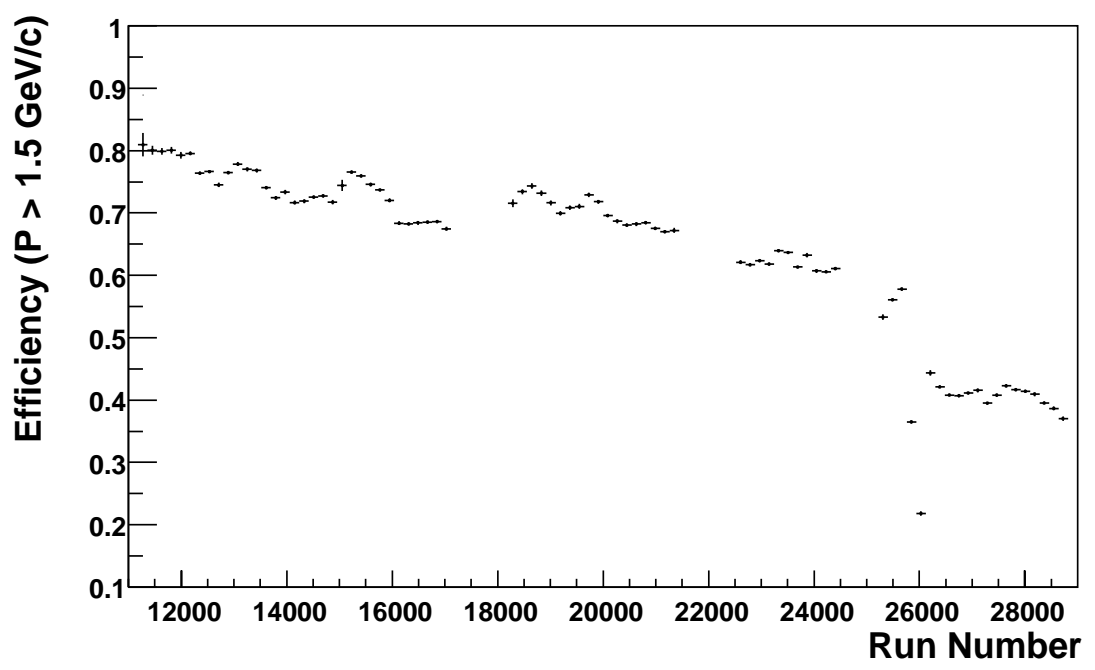

Figure 4.12: Muon efficiency from January 2000 to July 2002 as a function of the run number. 
performance calculated by specific groups of collaborators. Higher level analysis tasks such as $B$ flavor tagging and vertexing are also provided by specific groups as a service to the collaboration.

\subsubsection{Variables}

A collection of variables, employed by most $B$ analyses, is implemented in Beta. In this section, we define and describe the subset used in this analysis.

\section{Kinematics}

The beam energy substituted mass $\left(m_{E S}\right)$ and the energy difference $(\Delta E)[48]$ are nearly orthogonal Lorentz-invariants which exploit the kinematic constraints of the $\Upsilon(4 s) \rightarrow B \bar{B}$ decay. For a $B$ meson with 4 -momenta $q_{B}^{*}=\left(E_{B}^{*}, \mathbf{p}_{\mathbf{B}}^{*}\right)$ in the center of mass $(\mathrm{CM})$ and $q_{B}=\left(E_{B}, \mathbf{p}_{\mathbf{B}}\right)$ in the laboratory $(\mathrm{LAB})$ frames, these variables are defined as

$$
\begin{aligned}
m_{E S} & =\sqrt{q_{B}^{2}}, \text { and } \\
\Delta E & =\left(2 q_{B} q_{0}-4 E_{0}^{* 2}\right) / 4 E_{0}^{*},
\end{aligned}
$$

where $q_{0}^{*}=\left(E_{0}^{*}, p_{0}^{*}\right)$ and $q_{0}=\left(E_{0}, p_{0}\right)$ are the total 4 -momenta of the $e^{+} e^{-}$system in the $\mathrm{CM}$ and LAB frames, respectively. In the CM frame

$$
\begin{aligned}
m_{E S} & =\sqrt{\left(E_{0}^{*} / 2\right)^{2}-p_{B}^{* 2}}, \text { and } \\
\Delta E & =E_{B}^{*}-E_{0}^{*} .
\end{aligned}
$$

Since the $\Upsilon(4 s)$ mass is near the $B \bar{B}$ threshold, the center of mass momenta $p_{B}^{*}$ of the $B$ mesons are very small $(\approx 340 \mathrm{MeV} / c)$ when compared to the beam energy $E_{0}^{*}$ of more than $10.5 \mathrm{GeV}$. Therefore $p_{B}^{* 2}<<\left(E_{0}^{*} / 2\right)^{2}$ and the beam substituted mass is 
dominated by the beam energy resulting in a resolution of $\approx 2.6 \mathrm{MeV}$ which reflects the spread of the beam energy. In contrast $\Delta E$ distributions, which are dominated by the detector resolutions, are much wider. The standard implementations of these variables calculate $\Delta E$ in the $\mathrm{CM}$ frame and $m_{E S}$ in the LAB frame using

$$
m_{E S}=\sqrt{\left(\frac{1}{2} E_{0}^{*}+\mathbf{p}_{\mathbf{0}}^{*} \cdot \mathbf{p}_{\mathbf{B}}\right)^{2} / E_{0}^{2}-\left|\mathbf{p}_{\mathbf{B}}\right|^{2}}
$$

which only requires the momentum of the $B$ meson and does not depend on the masses of the decay products.

\section{Non-hadronic Backgrounds}

In addition to $B \bar{B}$ from $\Upsilon(4 S)$ decays, the $e^{+} e^{-}$collisions at PEP-II produce numerous final states which are potential backgrounds to $B$ decay analyses. The majority of the non-hadronic events are eliminated by requiring more than two good tracks $\left(N_{G T}\right)$ in an event ${ }^{1}$. In addition, the visible energy, defined as

$$
\mathcal{W}=\sum_{i}^{\text {GoodTracks }} \sqrt{m_{\pi}^{2}+p_{i}^{2}}+\sum_{j}^{\text {GoodNeutralClusters }} E_{j}
$$

also provides rejection of non-hadronic backgrounds. Figure 4.13 plots the distributions of these variables for the various physics processes at the $\Upsilon(4 S)$ energy. We will evaluate this selection for the $B^{0} \rightarrow h^{+} h^{--}$analysis in the next chapter.

\section{Hadronic Backgrounds and Event Shape}

Many variables which characterize the topology of events allow discrimination of signal $B \bar{B}$ from their hadronic backgrounds. Since the two $B$ mesons produced in $\Upsilon(4 s)$ decays are nearly at rest in the CM, they exhibit no correlation between the directions of their decay products. Meanwhile the small CM momentum of $B$ creates

\footnotetext{
${ }^{1}$ The good track and cluster definitions are listed in in sections 4.2.1 and 4.2.3, respectively.
} 

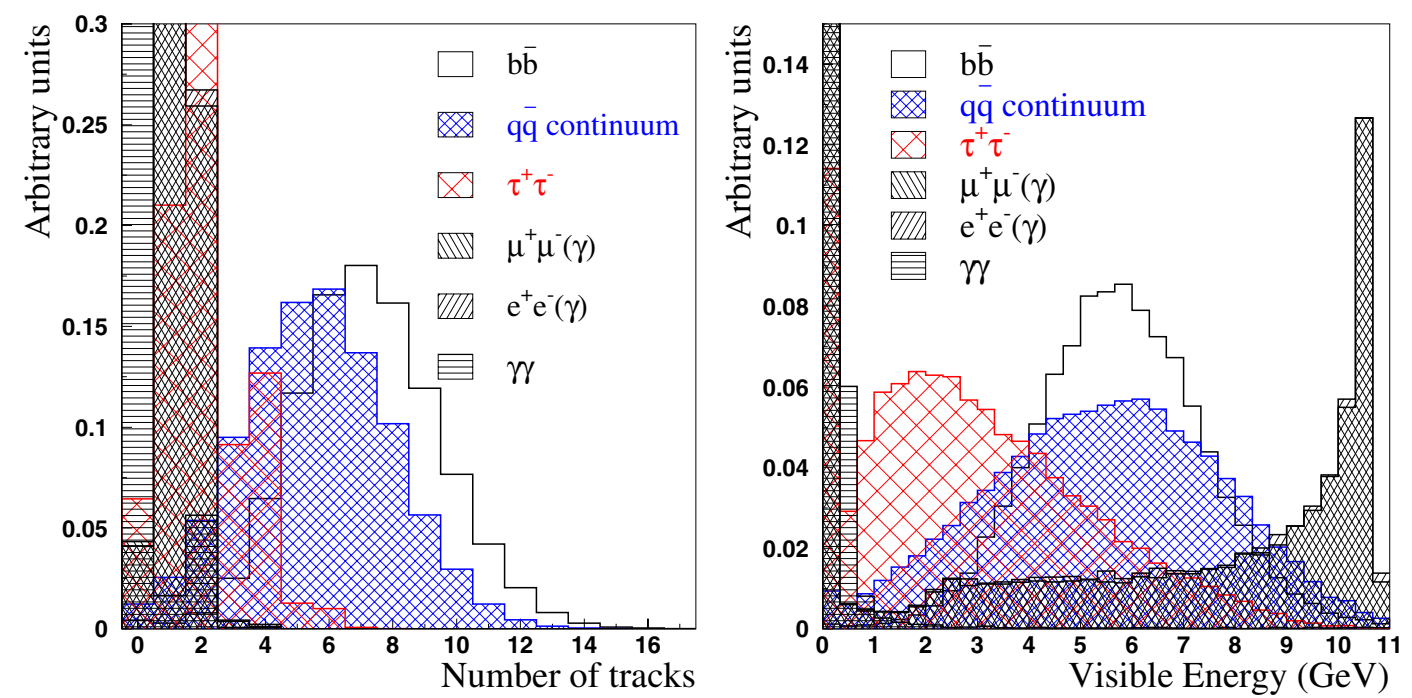

Figure 4.13: Distributions of (a) the number of tracks and (b) the visible energy $\mathcal{W}$, in the main physics processes at the $\Upsilon(4 S)$ energy. The distributions are from 
is reflected in the distribution of the magnitude of the cosine of this angle, $\left|\cos \theta_{S}\right|$, displayed in figure 4.14a for Monte Carlo samples of continuum and $B^{0} \rightarrow \pi^{+} \pi^{-}$ events, which is highly peaked at 1 for background and nearly flat for $B \bar{B}$ events. For this variable one axis is formed from a fully reconstructed two-body $B$ candidate while the remainder of the particles in the event, which are assumed to originate from the other $B$ meson, define the second axis. Also, the sphericity of an event, defined as sum

$$
S=\frac{3}{2}\left(\lambda_{2}+\lambda_{3}\right)
$$

of the two largest eigenvalues of $S^{\alpha \beta}$ calculated for all the particles of the event, provides additional discrimination of backgrounds. Figure 4.14b illustrates how the generally more directional background events have a lower sphericity distribution than $B \bar{B}$ events. The sphericity axis is often interchanged with the thrust axis $\hat{T}[50]$ which in practice provides nearly equivalent functionality. $\hat{T}$ is defined as the direction which maximizes the sum of their longitudinal momenta. $\left|\cos \theta_{T}\right|$ distributions, analogous to $\left|\cos \theta_{S}\right|$, are very similar to figure $4.14 \mathrm{a}$.

Expanding the spatial distribution of events in terms of Legendre polynomials produces another set of commonly used event shape discriminators, the Fox-Wolfram moments $H_{l}$ [51]. They are defined as

$$
H_{l}=\sum_{i, j} \frac{\left|\mathbf{p}_{i}\right|\left|\mathbf{p}_{j}\right|}{E_{T}^{2}} P_{l}\left(\cos \theta_{i j}\right),
$$

where $P_{l}$ are the Legendre polynomials, $\mathbf{p}_{i}$ are the particle momenta, $\theta_{i j}$ is the angle between particles $i$ and $j$, and $E_{T}$ is the total energy in the event. $H_{0}$ is constrained by energy-momentum conservation to be 1 when particle masses are ignored. For events with two jets of particles, $H_{1}=0$ and the remainder of the moments are $\approx 1$ for even $l$ and $\approx 0$ for odd $l$. Of these variables, the ratio $R_{2} \equiv H_{2} / H_{0}$, shown in 

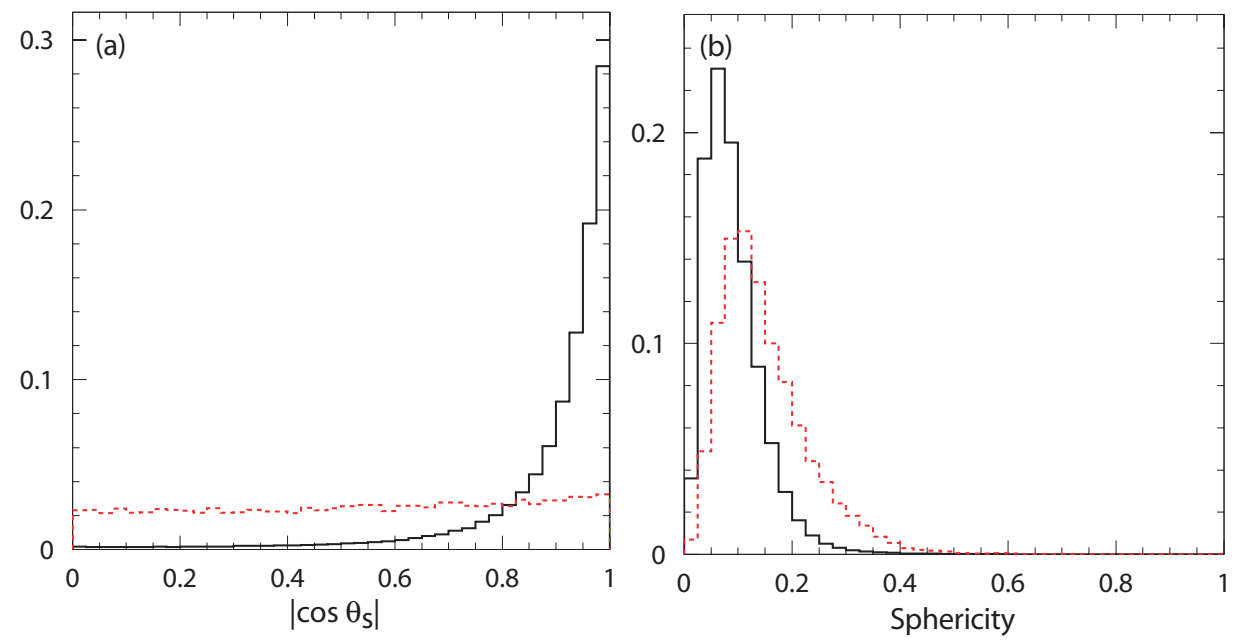

Figure 4.14: (a) The $\left|\cos \theta_{s}\right|$ and (b) sphericity distributions for $B \bar{B}$ events with a $B^{0} \rightarrow \pi^{+} \pi^{-}$decay (dashed red) and continuum background (solid black) events. The distributions are from simulated events and are normalized to unit area.

figure 4.15 is the most commonly used discriminator of $B \bar{B} /$ continuum events.

\subsubsection{Measurement of Number of $B \bar{B}$ Events}

Branching fraction measurements require knowledge of the total number of $B$ mesons in our dataset. BABAR determines this quantity by attributing the increase in the rate of hadronic events from the off to the on resonance data to $\Upsilon(4 S)$ decays. The number of $B \bar{B}$ events may then be measured using

$$
N_{B \bar{B}}=\frac{1}{\epsilon_{B \bar{B}}}\left(N_{\mathrm{H}}^{\mathrm{on}}-\frac{N_{\mu \mu}^{\mathrm{on}}}{N_{\mu \mu}^{\mathrm{off}}} \cdot \kappa \cdot N_{\mathrm{H}}^{\mathrm{on}}\right),
$$

where

- $N_{H}^{\text {on,off }}$ are the number of events satisfying a hadronic selection in each sample,

- $\epsilon_{B \bar{B}}$ is the efficiency of that selection on $B \bar{B}$ events, 


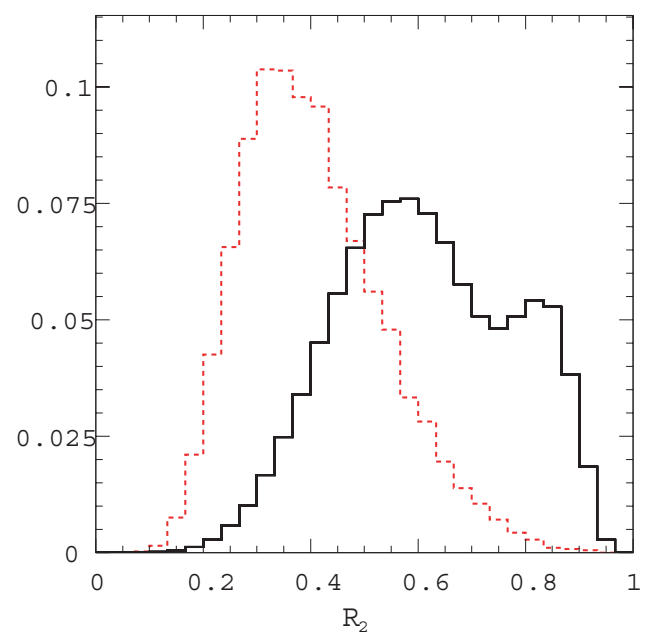

Figure 4.15: The $R_{2}$ distribution for signal $B^{0} \rightarrow \pi^{+} \pi^{-}$(dashed red) and continuum background (solid black) events. The distributions are from simulated events and normalized to unit area.

- $\kappa \approx 1$ accounts for differences in continuum cross-section and efficiencies at the different energies, and

- the ratio $\frac{N_{\mu \mu}^{\text {on }}}{N_{\mu \mu}^{\text {of }}}$ of the observed number of $e^{+} e^{-} \rightarrow \mu^{+} \mu^{-}$accounts for the difference in the collected luminosity.

This relation assumes that $\mathcal{B}(\Upsilon(4 S)) \rightarrow B \bar{B}=1$, which is a reasonable approximation [52].

The hadronic events are identified by requiring $N_{G T}>2, \mathcal{W}>4.5 \mathrm{GeV}, R_{2}<0.5$, and that the best vertex made from all the tracks in the event are within $5 \mathrm{~mm}$ in the $x-y$ plane and $6 \mathrm{~cm}$ in $z$ from the beam-spot. The $B \bar{B}$ efficiency of this selection is determined on simulated events, where careful attention has been made to confirm that all variable distributions are well reproduced [53]. The measured number of $B \bar{B}$ 
events in the data-sample used in this dissertation is

$$
N_{B \bar{B}}=(87.9 \pm 1.0) \times 10^{6},
$$

where the error is dominated by the uncertainty on the efficiency of the number of tracks $\left(N_{G T}\right)$ selection. 


\section{Chapter 5}

\section{Analysis Overview}

In the summer of 2000, SLAC's Asymmetric B-Factory presented several important analyses at the $\mathrm{XXX}^{\text {th }}$ International Conference on High Energy Physics (ICHEP) in Osaka, Japan [54]. Much of the excitement focused on the extraction of $\sin 2 \beta$ with the time-dependent $\mathrm{CP}$ analysis of the $B^{0} \rightarrow J / \psi K_{s}$ decay. A second wave of interest, fueled by the implications of CLEO's finding that $B^{0} \rightarrow K^{+} \pi^{-}$occurred more frequently than $B^{0} \rightarrow \pi^{+} \pi^{-}$[56] (see chapter 2), was focused on the two-body charmless $B$ decay branching fractions. $B A B A R$ 's preliminary analysis advertised its intention to measure $\sin 2 \alpha$ while also providing the opportunity to demonstrate the superiority of its Cherenkov particle identification system by improving on the CLEO measurement.

After the conference at Osaka, the team performing this analysis submitted the branching fractions measurement for publication in May of 2001 [58] and shifted focus to extracting $\sin 2 \alpha_{\mathrm{Eff}}$. This effort lead to the first extraction of time-dependent CP asymmetries in a rare mode, which was presented at the Summer 2001 LeptonPhoton Conference in Rome [59] and published later in the year [60]. The branching fractions and CP asymmetries were updated for Rencontres de Moriond in March of 
2002 [61]. This dissertation details the most recent measurement of these quantities, first presented in the summer of 2002 at ICHEP [62] and published in December $2002[63]$.

In this chapter we will detail the issues that shaped the analysis of $B^{0} \rightarrow h^{+} h^{\prime-}$ and eventually outline its blueprint. As in many BABAR analyses, some of the techniques employed to study these rare two-body $B$ decays were inspired by original work by the CLEO collaboration [64]. Their technique of extracting branching fractions of rare decays through simultaneous maximum likelihood fits using kinematic, multivariate background suppression, and particle identification information has been the basic analysis method since BABARś first measurement of the branching fractions of these modes. However, PEP-II's asymmetry and BABAR's DIRC required additional analysis inputs, and eventually the techinque was expanded with flavor tagging and vertexing information to yield the first time-dependent $\mathrm{CP}$ analysis in these $B$ decay modes.

\subsection{The Task at Hand}

The isolation of $B^{0} \rightarrow h^{+} h^{-}(h=\pi$ or $K)$ decays for branching fraction and timedependent $\mathrm{CP}$ asymmetry measurements is complicated by the rarity of decays, the significant background rate, and difficulties in separating pions and kaons. In this section we will detail the origins of these issues and discuss the handles developed to address them. 

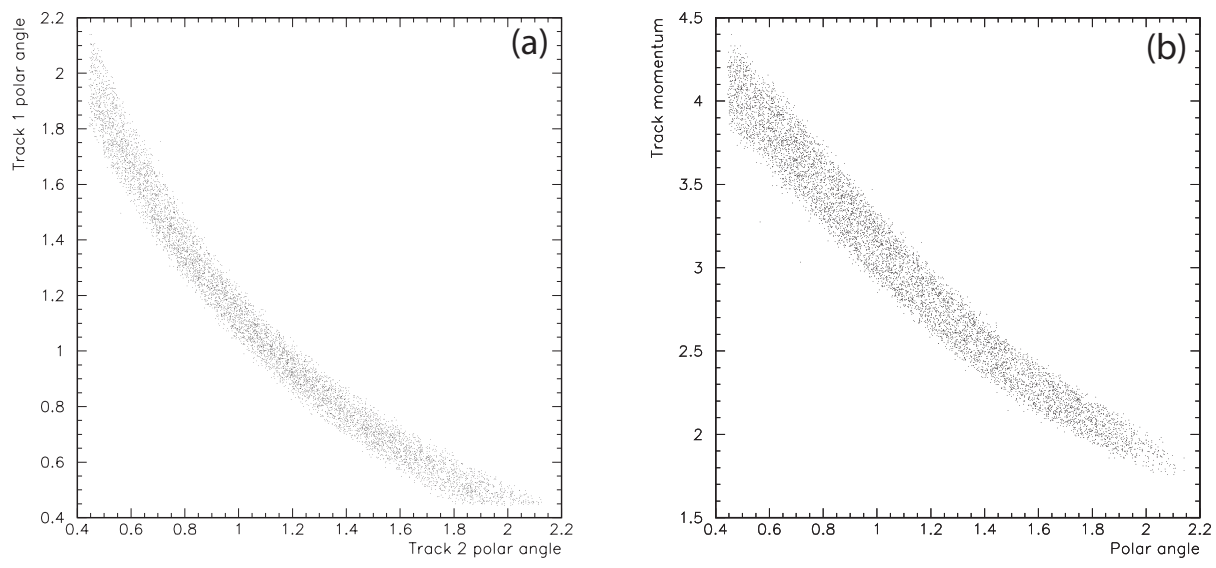

Figure 5.1: The polar angle $(\theta)$ of one track in $B^{0} \rightarrow h^{+} h^{\prime-}$ decays versus the polar angle of the (a) other track and (b) the track momentum.

\subsubsection{Kinematics}

The final state hadrons originating from the decay $B^{0} \rightarrow h^{+} h^{\prime-}$ are back-to-back and monochromatic in the $B$ meson decay frame, carrying momenta of $2.6 \mathrm{GeV} / c$ in opposite directions. The boost induced by PEP-II's asymmetry smears the track momenta to a roughly flat distribution between 1.7 and $4.2 \mathrm{GeV} / c$, but still preserves the kinematic correlation between the two hadrons (see figure 5.1a). This boost also introduces track polar angle $(\theta)$ and momentum correlations which are displayed in figure $5.1 \mathrm{~b}$.

Searching for these decays begins with the collection of all events with combinations of oppositely charged track pairs whose 4-momenta add to produce roughly the $B$ meson invariant mass. Since no particle identification is employed at this level of reconstruction, the assumption that both tracks are pions simplifies the combinatorics of selecting the candidate decays. A large invariant mass window of $600 \mathrm{MeV}$ around 
the $B$ mass guarantees the inclusion of all two-body $B$ decays to charged pions and kaons and also provides large side-band regions for background studies.

Since the $m_{E S}$ is sensitive to beam energies but nearly independent of mass hypotheses, distributions of any $B$ decay reconstructed with tracks is expected to be identical to $B^{0} \rightarrow \pi^{+} \pi^{-}, K^{+} \pi^{-}$, and $K^{+} K^{-}$. Therefore we may derive understating of this variable from large samples of more abundant $B$ decays (see section 6.1.1). $\Delta E$, however, is sensitive to masses and tracking resolution, which leads to two important consequences. First, since we assume all tracks are pions, this variable is shifted by $\approx 42 \mathrm{MeV}$ for each kaon track that is mis-assigned the pion mass. This shift is exploited to aid in separation of signal modes ${ }^{1}$. Second, since the daughters of the signal two-body $B$ s are typically the highest momentum tracks of any $B$ meson decay, the resulting $\Delta E$ resolution is unique. Since no other $B$ decay approximates these events, we derive understanding of $\Delta E$ from the Monte Carlo simulation, which we validate through comparisons with data in other decays (see section 6.1.1).

\subsubsection{Backgrounds}

The predominant background source of $B^{0} \rightarrow h^{+} h^{\prime-}$ candidates is from mesons produced in the fragmentation from continuum $e^{+} e^{-} \rightarrow u \bar{u}, d \bar{d}, s \bar{s}, c \bar{c}$ processes. Partially reconstructed $B$ mesons are a negligible background (see section 5.3.2) due to the unique kinematics of our signal decays. Also, as we saw in section 4.3.1, non-hadronic background events are also easily recognized through track multiplicity, event shape, and particle identification and hence can be eliminated. In continuum events, a high momentum track from one fragmenting quark may be combined with

\footnotetext{
${ }^{1}$ In the section 6.1 .1 we will quantify the momentum dependence of the $\Delta E$ shift due to mass mis-assignment.
} 
a similar track from the anti-quark flying in the opposite direction, producing a fake $B^{0} \rightarrow h^{+} h^{--}$candidate with kinematics that is very similar to the signal. Extrapolations of the shape of these variables, from the sideband to regions where signal is expected, provide a means of estimating their yield.

As discussed in section 4.3.1, the majority of these continuum events carry characteristics which permit some discrimination from $B$ decays. Hence cuts on event shape variables such as sphericity, the Fox-Wolfram moments, and the angle between the candidate axis and the rest of the event eliminate a large fraction of the background candidates. We obtain further background suppression from a linear combination of several event shape variables,

$$
\mathcal{F}=\sum_{i=1}^{N} \alpha_{i} x_{i}
$$

called a Fisher Discriminant (FD) [65], optimized to separate continuum events from $B$ decays. Here, the coefficients $\alpha_{i}$ providing the maximal separation between a sample signal and background events are given analytically as

$$
\vec{\alpha}=\frac{N_{S} N_{B}}{N_{S}+N_{B}}\left(\vec{x}_{S}-\vec{x}_{B}\right)^{T} \mathbf{W}^{-1}
$$

where $N_{S}$ and $N_{B}$ are their respective number of events of each type, $x_{i}$ are the variables, and $\vec{x}_{S, B}$ are vectors of variable means for the signal and background events. $\mathbf{W}$ is the dispersion component of the covariance matrix

$$
W_{\mu \nu}=\frac{1}{n}\left(\sum_{i}^{N_{S}}\left(x_{i \mu}-\bar{x}_{S \mu}\right)\left(x_{i \nu}-\bar{x}_{S \nu}\right)+\sum_{i}^{N_{B}}\left(x_{i \mu}-\bar{x}_{B \mu}\right)\left(x_{i \nu}-\bar{x}_{B \nu}\right)\right) .
$$

The resulting discriminant exploits the correlations between the inputs to separate the two event types.

In order to build the optimal discriminant, we extract topological variables for large numbers of candidate $B^{0} \rightarrow h^{+} h^{\prime-}$ events from Monte Carlo $B^{0} \rightarrow \pi^{+} \pi^{-}$and 
$e^{+} e^{-} \rightarrow u \bar{u}, d \bar{d}, s \bar{s}, c \bar{c}$ samples. Many of these variables characterize spatial distributions of tracks and neutral clusters about the thrust axis of the candidate in the $\Upsilon(4 s)$ rest frame. The CLEO collaboration, in their analysis of these modes, used the energy flow into 9 concentric, mutually exclusive cones about this axis to define a FD [64]. The separation power of this discriminant was derived from the fact that the jetty background events had more particles flying at small angles to the $B$ candidate daughters tracks, while signal decays had no such correlation. Extensive studies at BABAR indicated that CLEO's inputs provided nearly optimal signal/background separation [66]. Ultimately however, we choose another discriminant, composed of a simpler choice of variables which exploit the same information, due to its slightly better background rejection and aesthetic appeal. This new FD is composed of two variables $\left\{L_{0}, L_{2}\right\}$ defined as

$$
L_{i}=\sum_{j}^{\mathrm{ROE}} p_{j}\left|\cos \theta_{j}\right|^{i}
$$

where the sum includes all tracks and neutrals not in the $B$ candidate (often referred to as the rest-of-the-event (ROE)) and $\theta_{j}$ is the angle between particle's momentum direction and the axis defined by the $B$ 's two back-to-back daughter tracks. Distributions of the resulting discriminant, defined by

$$
\mathcal{F}=0.5139-0.6023 L_{0}+1.2698 L_{2}
$$

is displayed in figure 5.2 for signal and background events with $\left|\cos \theta_{S}\right|<.8$. Note that such event shape FDs have strong correlations with most topological variables so the degree of signal/background separation depends strongly on cuts on $\cos \theta_{S}$, sphericity, and $R_{2}$. 


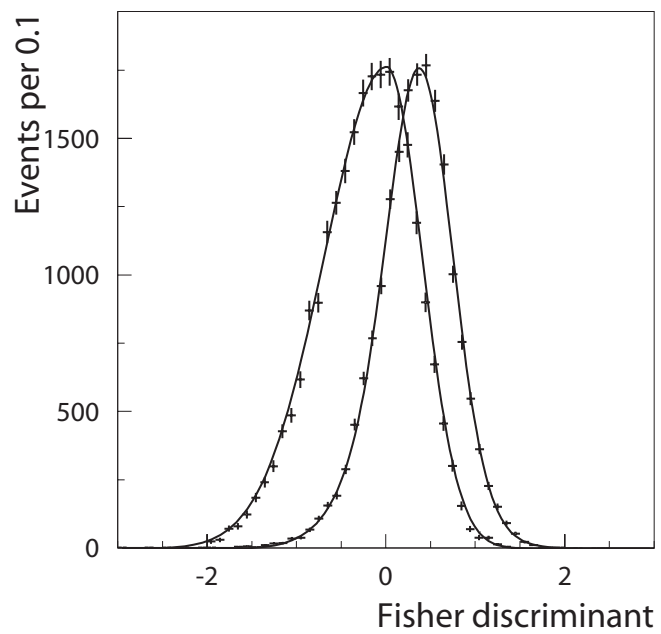

Figure 5.2: The Fisher Discriminant distribution for signal Monte Carlo (left) and sideband background (right). The fits to these are a bifurcated Gaussian for signal and double-Gaussian for background.

\subsubsection{Separation of Signal Modes}

The four 2-track combinations, $\pi^{+} \pi^{-}, K^{+} \pi^{-}, \pi^{+} K^{-}$, and $K^{+} K^{-}$, which we have collectively designated as "the signal" are difficult to distinguish and hence are backgrounds to one another. Measuring the branching fraction for $B$ decays to each of these final states necessitates separating them. The kinematic difference between the $\pi \pi, K \pi$, and $K K$ combinations provides a weak handle on the particle content of the candidate $B$ decays and negligible separation between $K^{+} \pi^{-}$and $\pi^{+} K^{-}$(see section 6.1.1). The high momenta of these tracks also limit the viability of the SVT and $\mathrm{DCH} d E / d x$ measurements as pion/kaon discriminators for these signal decays. Therefore DIRC $\theta_{c}$ measurement will serve as our only particle identification tool, dominating the separation of the signal modes.

Parameterizations of measured $\theta_{c}$, obtained from highly pure data control sam- 

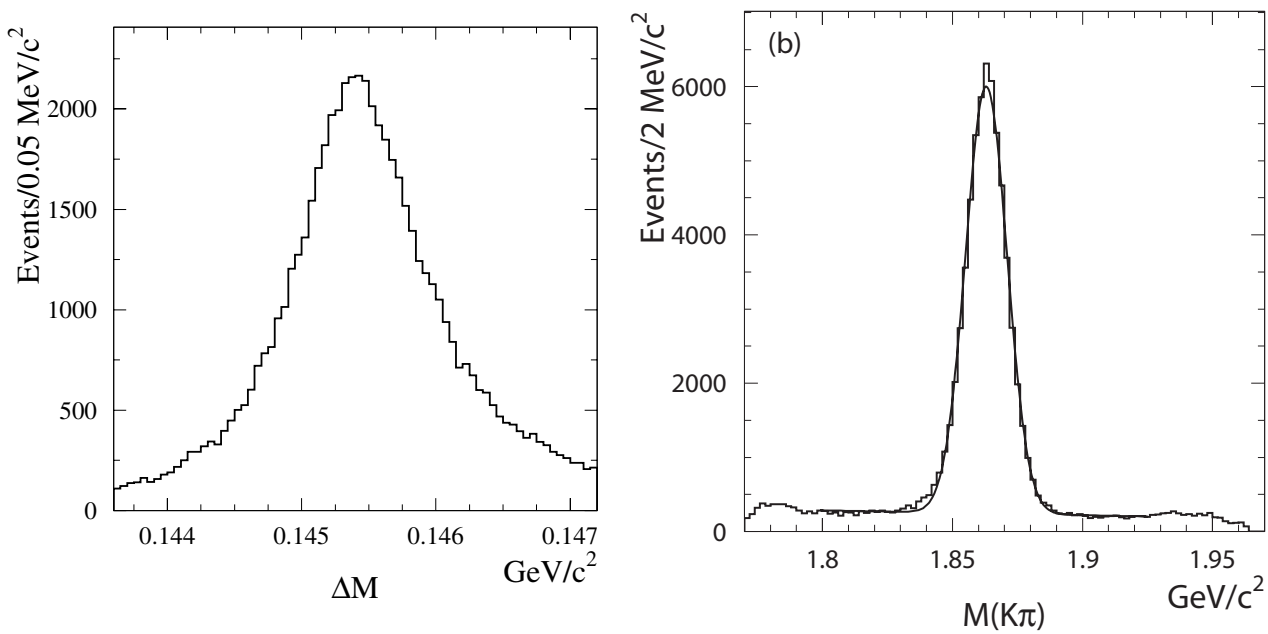

Figure 5.3: The (a) $\Delta M=M\left(D^{*}\right)-M\left(D^{0}\right)$ mass difference and (b) $D^{0}$ mass in the control sample used for studying DIRC $\theta_{c}$ measurements.

ples of charged pions and kaon, were used to calculate $\pi$ and $K$ likelihoods of each track. We obtained these samples by reconstructing the decay chain $D^{*+} \rightarrow D^{0} \pi^{+} \rightarrow$ $\left(K^{-} \pi^{+}\right) \pi^{+}$using only kinematic information and no particle identification. We build $D^{* \pm}$ candidates through four-momentum addition of tracks with appropriate mass assignment defined by the charge of the slow pion from the $D^{*}$ decay. A two standard deviation cut on the mass difference of the $D^{*}$ and $D^{0}\left(\sigma_{\Delta M}=0.9 \mathrm{MeV} / c^{2}\right.$, see figure 5.3a), which is measured well due to the small momentum of the slow pion, removes most mis-reconstructed $D^{0}$ s. Requiring that the $D^{0}$ momentum in the CM frame is $>2.5 \mathrm{GeV} / c$ isolates the continuum $D^{*}$ candidates and eliminates $B \bar{B}$ events which typically have higher combinatoric backgrounds. Removing events where the kaon track is in line with the $D^{0}$ flight direction in the $D^{0}$ rest frame provides further suppression of the combinatoric background. The cosine of this angle $\cos \theta_{K}^{*}$ is required to be $<0.8$. These selections produce a $D^{0}$ sample which has a purity of $\approx 96 \%$ (see figure $5.3 \mathrm{~b}$ ). 
The most recent study of this control sample calibrates the DIRC response by separately parameterizing the $\theta_{c}$ resolution, systematic bias, and charge dependence of the measured $\theta_{c}$ of kaons and pions. We only consider tracks which have sufficient signal Cherenkov photons $\left(N_{\gamma}>4\right)$ for a good $\theta_{c}$ measurement. In addition, since these tracks do not exhibit the momentum-polar angle dependence of two-body $B$ decays, we select the subset which fall within the band displayed in figure $5.1 \mathrm{~b}$ in order to mimic the $B^{0} \rightarrow h^{+} h^{\prime-}$ kinematics. These selections yield 32,074 kaon and 26,736 pion candidate tracks ${ }^{2}$. We separately study the $\pi^{+}, \pi^{-}, K^{+}$, and $K^{-}$distributions of the difference between the measured and expected $\theta_{c}, \Delta \theta_{c}=\theta_{c}-\theta_{c}^{\operatorname{Exp}}$, in bins of track polar angle $(\cos \theta)$. In each bin, $\Delta \theta_{c}$ of events within three standard deviations $\left(\sigma_{M_{D^{0}}}=8 \mathrm{MeV} / c^{2}\right)$ of the $D^{0}$ mass are background subtracted using events in the mass sideband. We fit the resulting distributions to Gaussian functions to obtain the bias and resolution of the $\theta_{c}$ measurements. Figure 5.4 displays the $\cos \theta$ dependence of the measured bias, $\mu_{\pi, K}^{ \pm}(\cos \theta)$, and resolution, $\sigma_{\pi, K}^{ \pm}(\cos \theta)$.

No dependence on other track parameters is observed when this calibration is applied back to the control sample. In particular, the corrected pull distributions, which are defined as $\left(\theta_{c}-\theta_{c}^{\operatorname{Exp}}-\mu_{\pi, K}^{ \pm}(\cos \theta)\right) / \sigma_{\pi, K}^{ \pm}(\cos \theta)$, are all centered at 0 with width of 1 and show no dependence on momentum (see figure 5.5). Pull distributions for the whole sample, which provide the likelihoods for discriminating pions and kaons (see section 6.1.1), are displayed in figure 5.6.

\footnotetext{
${ }^{2}$ The asymmetry in the yields is due the difference in $\pi / K$ mass, which propagates into a difference in selection efficiency for the momentum cut.
} 

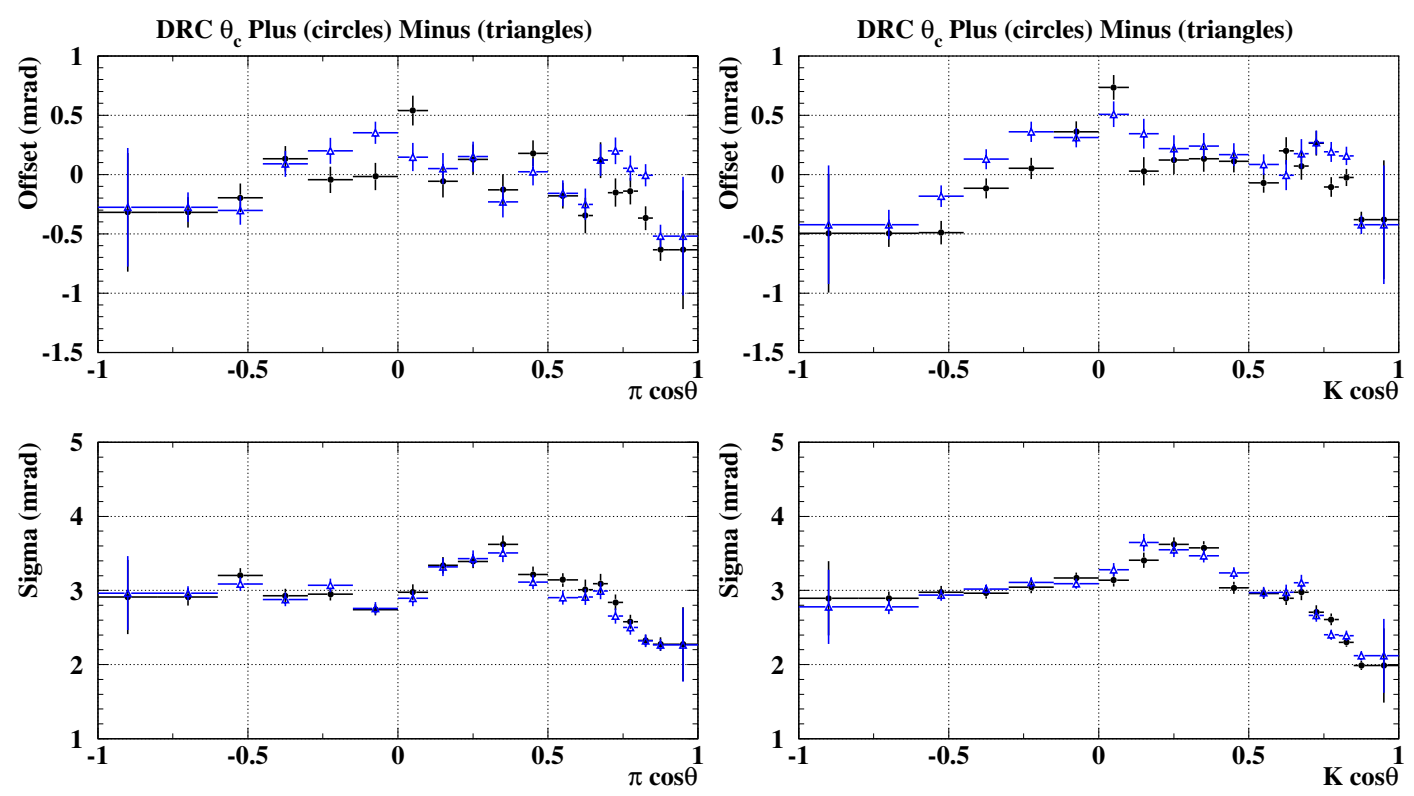

Figure 5.4: The measured $\theta_{c}$ resolution $\left(\sigma_{\pi, K}^{ \pm}(\cos \theta)\right)$ and bias $\left(\mu_{\pi, K}^{ \pm}(\cos \theta)\right)$ for positively (circles) and negatively charged (triangles) pions (left) and kaons (right). 

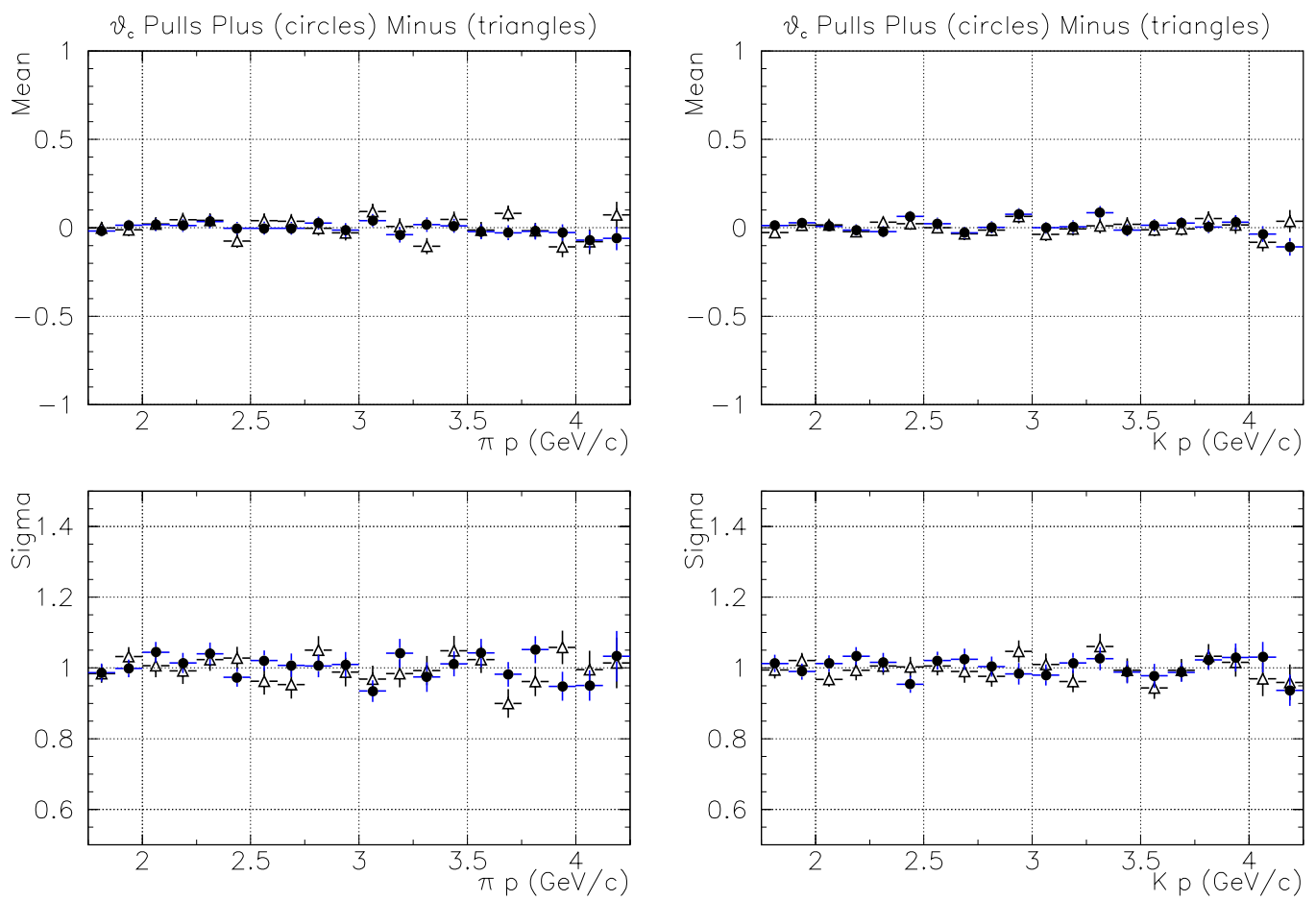

Figure 5.5: The mean and sigma of corrected $\theta_{c}$ pull distributions for positively (circles) and negatively (triangles) charged pions (left) and kaons (right) in bins of track momentum. The fits are to double Gaussian functions. 

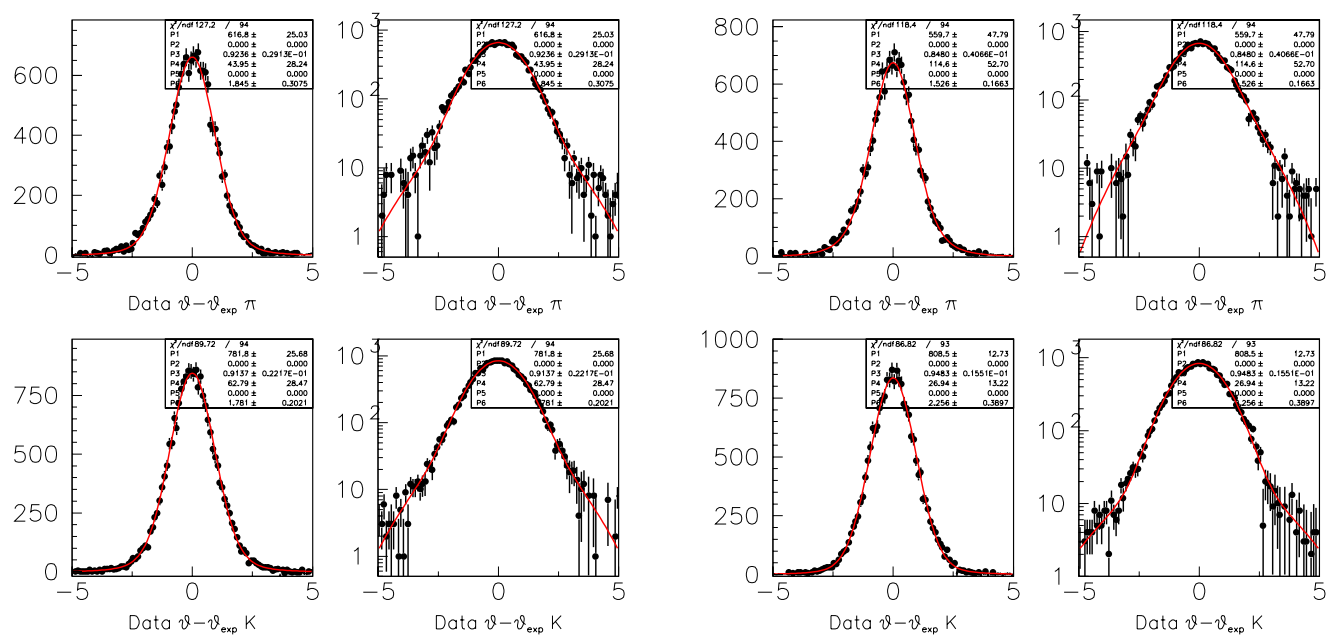

Figure 5.6: The corrected $\theta_{c}$ pull distributions for positively (left) and negatively (right) charged pions (top) and kaons (bottom). The fits are to double Gaussian functions.

\section{$5.2 \quad$ Extracting the Measurements}

As just discussed, our $B^{0} \rightarrow h^{+} h^{--}$signal events are embedded within a large background of $e^{+} e^{-} \rightarrow u \bar{u}, d \bar{d}, s \bar{s}, c \bar{c}$. Meanwhile our kinematic, event shape, and particle identification measurements permit the classification of the data into eight species of events: the four signal and four background $\pi^{+} \pi^{-}, K^{+} \pi^{-}, \pi^{+} K^{-}$, and $K^{+} K^{-}$final states. One method of isolating every signal mode would be to perform sets of cuts which are most efficient for each species, separating the candidate events into enriched subsamples. Extracting the branching fractions would then involve measuring the yields in every subsample and using a matrix of efficiencies for every species and selection to unfold the results. Typically the cuts employed in these methods are determined through the optimization of the statistical significance of the measurements, which generally scales as $N_{s} / \sqrt{\left(N_{s}+N_{b}\right)}$, where, in order to avoid bias, the signal 
and background yields $N_{s}$ and $N_{b}$ are derived from Monte Carlo, sidebands, and theoretical or previous yield estimates. In instances when the influence of backgrounds on a measurement is not well understood, the cuts may be chosen to remove the background instead. Since such cut-and-count or cut-and-fit methods typically discard the fraction of signal events with ambiguous classification, they are most attractive when the sacrifices in signal efficiencies are warranted by large signal purity and abundant signal yields.

We choose to extract measurements using a statistical technique known as Maximum Likelihood (ML) fitting which employs parameterizations of the variable distributions to separate the species and fit for the interesting quantities. This method provides important benefits to the analysis:

- ML fits are mathematically proven to provide the most statistically significant measurement attainable on any sample. Hence stringent selections are not required, signal efficiencies are high, and every event contributes to the measurement.

- All measurements are derived in one step providing both technical and procedural advantages.

- Simultaneous fitting of multiple samples provides better and easier assessments of systematic uncertainties. This benefit is reaped in two distinctive ways. First, when large signal-free side-band regions are included in the range of every variable, the fit projects the background contributions into the signal region. Consequently when the background parameterizations are determined by the fit, their systematic uncertainties are reflected in the statistical error of the measurement. Second, the simultaneous extraction of necessary parameters (for 
example resolution function parameters) through the inclusion of supplementary control samples assures the correct and easy propagation of the systematic uncertainties of those parameters to the final results.

When properly constructed, ML fits can in one step provide the most statistically significant measurement possible with small, yet accurate systematic error estimates. The difficulty lies in the complexity of such fits, which in our case will eventually extract 6 measurements and 113 parameters from two samples of nearly 100,000 events. Also such fits require understanding of the underlying physics and extensive validation studies. The latter was achieved through fits to thousands of toy Monte Carlo (toy MC) experiments which provide a fast and controlled means of simulating the fit inputs. For this analysis, these studies ultimately provide the quantitative and qualitative understanding of the expected behavior of the fit and bounds on the uncertainties due to various assumptions. They will be detailed in the next three chapters as we document the various components of the fit.

\subsubsection{Maximum Likelihood Fitting}

Given the probability distribution function (PDF) $P$ for an observable $x$, the likelihood of extracting the $N_{T}$ measurements $\left\{x_{1}, x_{2}, \ldots, x_{N}\right\}$ is defined as the product

$$
L\left(\alpha_{j}\right) \equiv \prod_{i}^{N} P\left(x_{i} \mid \alpha_{j}\right)
$$

of the probabilities for the individual measurements, where $\alpha_{j}$ parameterize $P$. For example, if $x$ is Gaussian distributed, $\left\{\alpha_{j}\right\}$ will be the mean and width of $P$. Maximizing $L$ with respect to $\alpha_{j}$ provides an unbiased estimator of $\alpha_{j}$ which is in general unique, sufficient (i.e. using all available information), and efficient (i.e. carrying 
the smallest possible variance). We will derive our measurements by applying this theorem.

The likelihood that describes the events in our sample of $B^{0} \rightarrow h^{+} h^{\prime-}$ candidates is obtained through a few modification to 5.1. First, we would like to simultaneously fit several variables distributions, specifically the variables $\left\{x^{k}\right\}=\left\{m_{E S}, \Delta E\right.$, a fisher discriminant $\mathcal{F}$, the $\theta_{c}$ for 2 tracks, and eventually flavor tagging and vertexing variables\}. If these variables are uncorrelated, the probability of obtaining an event with a specific set of measurements is simply the product of the individual probabilities

$$
P\left(\left\{x^{k}\right\}\right)=P\left(m_{E S}\right) P(\Delta E) \ldots
$$

Next, since eight different species of events may populate our sample, we expand the total probability of observing an event to

$$
\begin{aligned}
P\left(\left\{x^{k}\right\}\right)= & f_{\pi \pi}^{S} P_{\pi \pi}^{S}\left(\left\{x^{k}\right\}\right)+f_{K \pi}^{S} P_{K \pi}^{S}\left(\left\{x^{k}\right\}\right)+f_{\pi K}^{S} P_{\pi K}^{S}\left(\left\{x^{k}\right\}\right)+f_{K K}^{S} P_{K K}^{S}\left(\left\{x^{k}\right\}\right) \\
+ & f_{\pi \pi}^{B} P_{\pi \pi}^{B}\left(\left\{x^{k}\right\}\right)+f_{K \pi}^{B} P_{K \pi}^{B}\left(\left\{x^{k}\right\}\right)+f_{\pi K}^{B} P_{\pi K}^{B}\left(\left\{x^{k}\right\}\right)+f_{K K}^{B} P_{K K}^{B}\left(\left\{x^{k}\right\}\right),
\end{aligned}
$$

where the $f_{l}^{S, B}$ are the fractions of signal and background for the different final states, and the $P_{l}\left(\left\{x^{k}\right\}\right)$ are the corresponding products of the $\left\{x^{k}\right\}$ probability distributions for that final state. The resulting likelihood

$$
L\left(\alpha_{j}, f_{l}^{S, B}\right)=\prod_{i}^{N_{T}} P\left(\left\{x^{k}\right\}_{i} \mid \alpha_{j}, f_{l}^{S, B}\right)
$$

now fully describes our data sample. A closer look into this likelihood reveals that the use of fractions does not take into account the Poisson statistics governing the sample yield. In fact equation (5.2), in its current form, has no constraint enforcing the fractions sum to 1 . We remedy these issues by re-parameterizing (5.2) in terms of yields $N_{l}$ by the substituting $f_{l}=N_{l} / \sum_{l^{\prime}}^{8} N_{l^{\prime}}$, and extending the likelihood by 
multiplying a Poisson term $\frac{N_{T} N_{T}^{\prime} e^{-N_{T}^{\prime}}}{N_{T} !}$, where $N_{T}^{\prime}=\sum_{l} N_{l}$. After simplification, the final likelihood takes the form

$$
\begin{aligned}
& L\left(\alpha_{j}, N_{l}^{S, B}\right)=\frac{e^{-\sum_{l} N_{l}}}{N_{T} !} \prod_{i}^{N_{T}}\left\{N_{\pi \pi}^{S} P_{\pi \pi}^{S}\left(\left\{x^{k}\right\}_{i}\right)+N_{K \pi}^{S} P_{K \pi}^{S}\left(\left\{x^{k}\right\}_{i}\right)+N_{\pi K}^{S} P_{\pi K}^{S}\left(\left\{x^{k}\right\}_{i}\right)\right. \\
& +N_{K K}^{S} P_{K K}^{S}\left(\left\{x^{k}\right\}_{i}\right)+N_{\pi \pi}^{B} P_{\pi \pi}^{B}\left(\left\{x^{k}\right\}_{i}\right)+N_{K \pi}^{B} P_{K \pi}^{B}\left(\left\{x^{k}\right\}_{i}\right) \\
& \left.+N_{\pi K}^{B} P_{\pi K}^{B}\left(\left\{x^{k}\right\}_{i}\right)+N_{K K}^{B} P_{K K}^{B}\left(\left\{x^{k}\right\}_{i}\right)\right\} .
\end{aligned}
$$

In addition to the measurements we are interested in, the variables $\alpha_{j}$ and $N_{l}^{S, B}$ include background yields and PDF parameters. When maximizing this likelihood, we will choose a select set of these parameters to be determined from our sample. Typically these parameters will include background PDF parameters which this likelihood will describe well due to the inclusion of large sideband regions. Signal PDF parameters will be generally extracted from control samples of more abundant $B$ decays than our signal. When possible, we will multiply the likelihood which extracts these signal parameterizations to (5.3), therefore extracting the parameters simultaneously. An estimator of the quality of the fit may be determined from $\chi^{2}=-2 \log L+$ $C\left(N_{T}^{\prime}\right)$, where $C\left(N_{T}^{\prime}\right)$ is an arbitrary constant which is dependent on the sample size. Since $L$ and $\log L$ share the same maxima, we choose to minimize $\chi^{2}$ when performing the fit. This is a standard trick which allows the estimation of the errors on our fitted parameters from the derivatives used to minimize the $\chi^{2}$, and also provides a more computationally manageable task by converting the large product in (5.3) to a sum. Finally, large samples of toy MC experiments will provide the distribution of $\chi^{2}$, allowing the estimation the goodness-of-fit. 


\subsubsection{The LMinuit Fitting Package}

The development of a new fitting software for this analysis was warranted by the absence of a viable alternative during the preliminary stages of the investigation of these modes. In an effort to avoid building software capable of only a single task, a general interface to the Minuit minimization package was created. The resulting package, named LMinuit, provides users with a toolbox of commonly used functions, algorithms, and input/output and organization routines accessible through a full featured programming language based on LISP. LMinuit also handles the caching of function evaluations on the data, reducing the number of numerical calculation and shortening fit times by an order of magnitude. Also, specialized generators provide a means of producing sophisticated toy Monte Carlo experiments. A LISP library of support scripts provide object oriented tools for handling parameters and probability distribution functions (PDFs), writing the likelihoods, configuring and controlling the fit and toy MC, and producing ntuple and graphical output.

\subsubsection{The Toy Monte Carlo}

Toy Monte Carlos provide a fast means of generating events by reproducing variables employed in the analysis from parameterizations of their distributions derived from the data and the Geant4 Monte Carlo. The goal is to produce an output which, to the fit, is indistinguishable from the data. For variables which are considered to be uncorrelated to each-other, such as $m_{E S}$ and $\mathcal{F}$, generating the PDFs is a simple task. The parameterizations of the Cherenkov angles and $\Delta E$, however, depend on the kinematics of the decay. In particular two important features must be properly reproduced in order to accurately simulate the particle identification in the data: the track momentum/polar-angle $(\theta)$ dependence induced by the boost, because it 
is essential to model the DIRC resolution's sensitivity to $\cos \theta$; and the correlations between the two tracks in the decay, since if one track is fast and therefore difficult to distinguish between a kaon or pion, the other is slow and is easier to identify. Therefore this analysis warrants a full kinematic generator in the toy Monte Carlo. LMinuit reproduces the signal by first simulating the two body $B$ decay in its rest frame, creating two $2.6 \mathrm{GeV}$ back-to-back tracks flying in a random direction. Full Monte Carlo distributions of the $B$ flight angles and momenta $\left(p_{0}^{*}\right)$ are then used to boost the tracks into $\Upsilon(4 s)$ frame. Finally the system is again boosted into the lab frame and acceptance corrections are applied. Since the background continuum events which are considered by this analysis have kinematics which are similar to the signal decays, they are produced by the same algorithm, but with $p_{0}^{*}$ and angle distributions determined from sideband data.

\subsection{Analysis Outline}

The analysis method is essentially defined by our choice to extract yields through a maximum likelihood fit, which derives its ability to distinguish between the eight possible signal and background species of events populating our sample of $B^{0} \rightarrow h^{+} h^{\prime-}$ candidates from accurate parameterizations of kinematic, event shape, and particle identification variables. Since this technique promises to provide the most significant measurement of the branching fractions possible on any data sample, we choose our selections of candidates to eliminate only the most obvious background sources, providing a manageable data sample with the largest possible signal efficiencies to the fit. In this section we will detail this selection and calculate the signal efficiencies. In the next chapter we will focus on the parameterizations of the PDFs of the fit 
variables.

The extraction of time-dependent asymmetries in $B^{0} \rightarrow \pi^{+} \pi^{-}$from our data sample necessitates the addition of information on the reconstructed decay vertex and flavor of the second $B$ meson in the event to the maximum likelihood fit. We choose to extract signal yields without the added complications of the full CP analysis ${ }^{3}$. This choice is warranted by the opportunity to obtain a high precision $(\approx 5 \%)$ measurement of the $B^{0} \rightarrow K^{+} \pi^{-}$branching fraction from the full data sample (see introduction to chapter 8).

\subsubsection{Data Sets}

The data sets of of recorded and simulated events employed in this analysis are:

- Events recorded by the BABAR detector:

- On resonance: $81.0 \mathrm{fb}^{-1},(87.9 \pm 1.0)$ million $B \bar{B}$ pairs from

* Run 1 (1999-2000): $11.4 \mathrm{fb}^{-1}$ DCH HV=1960V, $8.8 \mathrm{fb}^{-1}$ DCH HV=1900V,

* Run 2 (2001-2002): $61.2 \mathrm{fb}^{-1}$ DCH HV=1900V.

- Off resonance: $9.6 \mathrm{fb}^{-1}$, from

* Run 1 (1999-2000): $1.6 \mathrm{fb}^{-1}$ DCH HV=1960V, $1.0 \mathrm{fb}^{-1} \mathrm{DCH} \mathrm{HV}=1900 \mathrm{~V}$,

* Run 2 (2001-2002): $7.0 \mathrm{fb}^{-1}$.

- Geant4 simulated events:

- Signal Monte Carlo:

\footnotetext{
${ }^{3}$ Though the branching fractions gain statistical precision from the background suppression provided by the vertexing and flavor tagging, we ignore these variables in order to avoid any possible systematic effects on the branching fractions. See the introduction to chapter 8 .
} 
* $B^{0} \rightarrow \pi^{+} \pi^{-}(99,000$ events $)$,

$* B^{0} \rightarrow K^{+} \pi^{-}(178,000$ events $)$,

$* B^{0} \rightarrow K^{+} K^{-}(77,000$ events $)$.

- Continuum/background Monte Carlo:

$* e^{+} e^{-} \rightarrow$ uds (147 million events, $70.5 \mathrm{fb}^{-1}$ ),

$* e^{+} e^{-} \rightarrow c \bar{c}\left(92.5\right.$ million events, $\left.71.2 \mathrm{fb}^{-1}\right)$,

$* e^{+} e^{-} \rightarrow \tau^{+} \tau^{-}\left(68.5\right.$ million events, $\left.72.8 \mathrm{fb}^{-1}\right)$.

$* e^{+} e^{-} \rightarrow e^{+} e^{-}$(1.7 million events).

- $B$ decay Monte Carlo:

* Generic $B$ decay:

- $B^{0} / \bar{B}^{0}$ (43.4 million, $\left.82.8 \mathrm{fb}^{-1}\right)$,

- $B^{+} / B^{-}\left(43.3\right.$ million, $\left.82.6 \mathrm{fb}^{-1}\right)$.

* $B$ decay to final states with no charm $^{4}$.

- $B^{0} / B^{+}\left(4.8\right.$ million, $\left.192 \mathrm{fb}^{-1}\right)$,

- $\bar{B}^{0} / B^{-}\left(4.8\right.$ million, $\left.192 \mathrm{fb}^{-1}\right)$.

* B Backgrounds:

- $B^{0} \rightarrow \rho^{+} \pi^{-}, \rho^{+} \rightarrow \pi^{+} \pi^{0}$ (63,000 events $)$.

- $B^{0} \rightarrow K^{*+} \pi^{-}, K^{*+} \rightarrow K^{+} \pi^{-}(37,000$ events $)$.

- $B^{0} \rightarrow K^{*+} \pi^{-}, K^{*+} \rightarrow K_{s}^{0} \pi^{+}(55,153$ events $)$.

- $B^{0} \rightarrow K^{* 0} \pi^{0}, K^{* 0} \rightarrow K^{+} \pi^{+}(38,000$ events $)$.

\footnotetext{
${ }^{4}$ In order to study $b$-quark backgrounds due to other rare modes these two samples were generated using the branching fractions and upper limits of all possible $B$ mesons decays to final states with no charm quarks.
} 


$$
\begin{aligned}
& \cdot B^{+} \rightarrow K^{*+} \pi^{0}, K^{*+} \rightarrow K^{+} \pi^{0}(38,000 \text { events }) . \\
& \cdot B^{+} \rightarrow K^{*+} \pi^{0}, K^{*+} \rightarrow K_{s}^{0} \pi^{+}(38,000 \text { events }) . \\
& \cdot B^{0} \rightarrow K^{* 0} \gamma, K^{* 0} \rightarrow K^{+} \pi^{-}(38,000 \text { events }) .
\end{aligned}
$$

\subsubsection{Selection Criteria and Efficiency}

Table 5.1 summarizes the selections criteria imposed on the data, listing the efficiency of each cut for each signal mode. In order to eliminate the majority of non-hadronic products of the $e^{+} e^{-}$collision (see table 5.2) which are typically two-prong, we search for $B^{0} \rightarrow h^{+} h^{\prime-}$ candidates in events which have three or more tracks reconstructed and $R_{2}<0.95$. The requirement that the sphericity of the event is $>0.01$ further reduces the number of $e^{+} e^{-} \rightarrow \tau^{+} \tau^{-}$events. Both of these variables are calculated using all tracks and neutral clusters in the event.

We construct candidates by finding the best common vertex for pairs of oppositely charged "good quality" tracks (see section 4.2.1), adjusting their momentum vectors to be consistent with production at the resulting vertex point. Simple four-vector addition of these momenta, with the assumption of pion mass for each particle, provides the four-momentum of the $B$ candidate which in conjunction with beam parameters provided by PEP-II allows the calculation of $m_{\mathrm{ES}}$ and $\Delta E$. We retain candidates in the region $|\Delta E|<0.420 \mathrm{GeV}$ and $5.2<m_{\mathrm{ES}}<5.2895 \mathrm{GeV} / c^{2}$ for our studies, but only consider the subset which satisfy $|\Delta E|<0.150 \mathrm{GeV}$ in the fit. This range includes nearly all signal decays, and eliminates almost all mis-reconstructed $B$ decays (such as the charmless decays to three-body final states in tables 5.3 and 5.4), which typically have a $\Delta E$ which is shifted to negative values by more than one pion mass.

Though the Fisher discriminant provides good background suppression to our fit, the requirement $\left|\cos \theta_{S}\right|<0.8$ provides additional continuum rejection by using event 
Table 5.1: Summary of detection efficiencies for $\pi^{+} \pi^{-}, K^{+} \pi^{-}$, and $K^{+} K^{-}$as determined in Geant4 signal Monte Carlo samples with about 40k events. The tracking correction accounts for measured tracking efficiency differences between simulated and recorded data. The efficiency of each cut is relative to the previous one and the errors are statistical, except for the PID and tracking efficiency corrections (which are included in the error on the total efficiency). For a description of each cut, refer to the text.

\begin{tabular}{|c|c|c|c|}
\hline & $\epsilon\left(\pi^{+} \pi^{-}\right)$ & $\epsilon\left(K^{+} \pi^{-}\right)$ & $\epsilon\left(K^{+} K^{-}\right)$ \\
\hline \multicolumn{4}{|l|}{ Standard Selection } \\
\hline \multicolumn{4}{|c|}{ Reconstruction $+R_{2}<0.95+$} \\
\hline$S+\left|\cos \theta_{S}\right|<0.9$ & $0.7101 \pm 0.0024$ & $0.6993 \pm 0.0025$ & $0.6835 \pm 0.0025$ \\
\hline Good track quality & $0.8907 \pm 0.0020$ & $0.8899 \pm 0.0020$ & $0.8881 \pm 0.0021$ \\
\hline$\Delta E-m_{\mathrm{ES}}$ & $0.9912 \pm 0.0006$ & $0.9888 \pm 0.0007$ & $0.9812 \pm 0.0009$ \\
\hline$\left|\cos \theta_{S}\right|<0.8$ & $0.8701 \pm 0.0023$ & $0.8773 \pm 0.0022$ & $0.8734 \pm 0.0024$ \\
\hline standard efficiency & $0.5455 \pm 0.0027$ & $0.5399 \pm 0.0027$ & $0.5202 \pm 0.0026$ \\
\hline \multicolumn{4}{|l|}{ PID Selection } \\
\hline$\theta_{c}$ & $0.8281 \pm 0.0027$ & $0.8284 \pm 0.0028$ & $0.8306 \pm 0.0029$ \\
\hline$N_{\gamma}$ & $0.9362 \pm 0.0019$ & $0.9324 \pm 0.0020$ & $0.9292 \pm 0.0021$ \\
\hline electron veto & $0.9998 \pm 0.0001$ & $1.0 \pm 0.0$ & $1.0 \pm 0.0$ \\
\hline proton veto & $0.9653 \pm 0.0015$ & $0.9652 \pm 0.0016$ & $0.9690 \pm 0.0015$ \\
\hline data/MC correction & $0.9800 \pm 0.0100$ & $0.9800 \pm 0.0100$ & $0.9800 \pm 0.0100$ \\
\hline PID efficiency & $0.7333 \pm 0.0081$ & $0.7306 \pm 0.0081$ & $0.7329 \pm 0.0082$ \\
\hline \multicolumn{4}{|l|}{$\Delta t$ Selection } \\
\hline$\Delta t$ & $0.9852 \pm 0.0010$ & $0.9879 \pm 0.0009$ & $0.9859 \pm 0.0010$ \\
\hline$\sigma_{\Delta t}$ & $0.9803 \pm 0.0011$ & $0.9784 \pm 0.0012$ & $0.9812 \pm 0.0012$ \\
\hline$\Delta t$ efficiency & $0.9658 \pm 0.0015$ & $0.9666 \pm 0.0015$ & $0.9674 \pm 0.0015$ \\
\hline nominal efficiency & $0.3863 \pm 0.0047$ & $0.3813 \pm 0.0047$ & $0.3688 \pm 0.0046$ \\
\hline tracking correction & $0.983 \pm 0.016$ & $0.983 \pm 0.016$ & $0.983 \pm 0.016$ \\
\hline Total Efficiency & $0.3798 \pm 0.0076$ & $0.3748 \pm 0.0076$ & $0.3623 \pm 0.0073$ \\
\hline
\end{tabular}


shape information not fully exploited by the Fisher discriminant ${ }^{5}$ (see table 5.5.). This selection, by eliminating roughly $50 \%$ of the continuum background while retaining $>87 \%$ of the signal, also provides an efficient means of reducing the data sample to a manageable size for the fit.

Since the fit predominantly relies on the $\theta_{c}$ measurements for kaon/pion separation, we only consider candidates which are in the DIRC acceptance and have good $\theta_{c}$ fits for both tracks. Studies of kaons and pions in the $D^{*}$ control sample indicate that at least 5 signal photons are required for a reliable $\theta_{c}$ extraction. Requiring that each track is within $4 \sigma$ of the expected pion or kaon $\theta_{c}$ rejects protons, which may originate from machine backgrounds or $e^{+} e^{-} \rightarrow$ baryons production. Electron rejection is also required due to the presence of $e^{+} e^{-} \rightarrow e^{+} e^{-}$events with additional tracks due to radiation from the final state electrons or the beam hallow or due to $\tau$ decays. We identify and reject tracks which are highly likely to be electrons when they are $4 \sigma$ and $3 \sigma$ consistent with electron hypothesis of the DCH $d E / d x$ and DIRC $\theta_{c}$ measurements, are well associated with an EMC cluster which exhibits appropriate shower shape, and satisfy the strict requirement of $0.89<E / p<1.2^{6}$. In order to correctly account for the kinematic effects, the efficiency of these requirements for signal is determined from Monte Carlo and corrected for inconsistencies with data through comparisons of data and simulated $D^{*}$ control samples.

Finally we apply requirements on the quality of the measured time difference

\footnotetext{
${ }^{5} \theta_{S}$ is the angle between sphericity axes of the $B$ candidate and the rest of the good tracks and neutrals in the event. See section 4.3.1.

${ }^{6}$ The $\mathrm{E} / \mathrm{p}$ distribution is centered at 1 (by virtue of the $\mathrm{E} / \mathrm{p}$ calibration) and has a width of $0.02-0.03$. The cut on the lower side is optimized so that the influence of radiation and energy loss in dead material in front of the EMC is minimized while keeping a good separation against hadrons. The upper cut is mainly used for the rejection of annihilating anti-protons.
} 

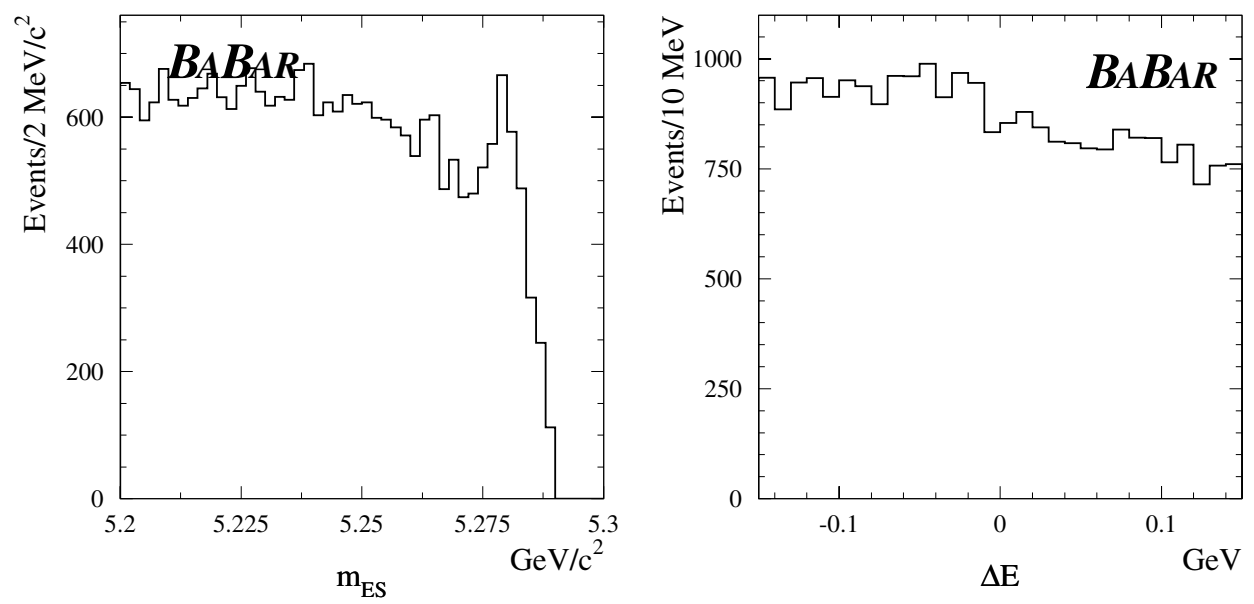

Figure 5.7: The $m_{\mathrm{ES}}$ and $\Delta E$ distributions of the 26,070 selected $B^{0} \rightarrow h^{+} h^{\prime-}$ candidates in the on-resonance sample.

$\Delta t$ between the decay of the two $B$ mesons in the event. Chapter 7 will provide a complete description of these variables as well as the evaluation of the efficiency of cutting on them. Despite the loss of efficiency, we retain these selections for extracting the branching fraction due to historical reasons which facilitated comparisons with the time-dependent analysis. Figure 5.7 displays the $m_{\mathrm{ES}}$ and $\Delta E$ distributions of the 26,070 $B^{0} \rightarrow h^{+} h^{-}$candidates selected in the on-resonance sample. 
Table 5.2: Selection efficiencies for non-hadronic $e^{+} e^{-} \rightarrow \tau \bar{\tau}$ and $e^{+} e^{-} \rightarrow e^{+} e^{-}$events from Geant4 Monte Carlo. The reconstruction efficiency is relative to the number of generated events in each Monte Carlo sample, and the efficiency of each successive cut is relative to the previous one. The "expected yield" is the estimated number of events of the specific background source in the $B^{0} \rightarrow h^{+} h^{\prime-}$ sample. In order to estimate the background contributions to each signal decay, the $\pi \pi, K \pi$, and $K K$ "select" entries reflect the number of events in each Monte Carlo background sample which are consistent with the specific signal final state. These events are selected using particle identification and three standard deviation cuts on $m_{\mathrm{ES}}$ and $\Delta E$. The listed efficiency for these entries is relative to the $\Delta t, \sigma_{\Delta} t$ cut.

\begin{tabular}{|c|c|c|c|c|}
\hline \multirow[b]{3}{*}{ Cut } & \multicolumn{2}{|r|}{$e^{+} e^{-} \rightarrow \tau \bar{\tau}$} & \multicolumn{2}{|c|}{$e^{+} e^{-} \rightarrow e^{+} e^{-}$(Bhabha) } \\
\hline & Events & & Events & \\
\hline & Passed & Efficiency & Passed & Efficiency \\
\hline Reconstruction & 105384 & $1.54 \cdot 10^{-3} \pm 4.74 \cdot 10^{-6}$ & 69 & $3.985 \cdot 10^{-5} \pm 4.79 \cdot 10^{-6}$ \\
\hline$N_{G T}>2, R_{2}<0.98$ & 35375 & $33.57 \% \pm 0.15 \%$ & 7 & $10.14 \% \pm 3.63 \%$ \\
\hline$R_{2}<0.95$ & 34480 & $97.47 \% \pm 0.08 \%$ & 4 & $57.14 \% \pm 18.70 \%$ \\
\hline$S>0.01$ & 32975 & $95.64 \% \pm 0.11 \%$ & 3 & $75.00 \% \pm 21.65 \%$ \\
\hline Good Track & 27212 & $82.52 \% \pm 0.21 \%$ & 2 & $66.67 \% \pm 27.22 \%$ \\
\hline$\Delta E-m_{\mathrm{ES}}$ & 8754 & $32.17 \% \pm 0.28 \%$ & 0 & $0.00 \% \pm 0.00 \%$ \\
\hline$\left|\cos \theta_{S}\right|<0.8$ & 2219 & $25.35 \% \pm 0.46 \%$ & 0 & $0.00 \% \pm 0.00 \%$ \\
\hline$\theta_{c}$ & 1719 & $77.47 \% \pm 0.89 \%$ & 0 & $0.00 \% \pm 0.00 \%$ \\
\hline$N_{\gamma}$ & 1676 & $97.50 \% \pm 0.38 \%$ & 0 & $0.00 \% \pm 0.00 \%$ \\
\hline Electron Veto & 1205 & $71.90 \% \pm 1.10 \%$ & 0 & $0.00 \% \pm 0.00 \%$ \\
\hline Proton Veto & 1194 & $99.09 \% \pm 0.27 \%$ & 0 & $0.00 \% \pm 0.00 \%$ \\
\hline$\Delta t, \sigma_{\Delta t}$ & 563 & $47.15 \% \pm 1.44 \%$ & 0 & $0.00 \% \pm 0$ \\
\hline Total Selection Eff & & $8.22 \cdot 10^{-6} \pm 3.47 \cdot 10^{-7}$ & & $0 \pm 0$ \\
\hline Luminosity $\left(\mathrm{fb}^{-1}\right)$ & 71.2 & & & \\
\hline Expect Yield (Events) & & $625.56 \pm 0.52$ & & $0.00 \pm 0.00$ \\
\hline$\pi \pi$ Select & 25 & $4.44 \% \pm 0.87 \%$ & 0 & $0.00 \% \pm 0.00 \%$ \\
\hline$K \pi$ Select & 5 & $0.89 \% \pm 0.40 \%$ & 0 & $0.00 \% \pm 0.00 \%$ \\
\hline$K K$ Select & 0 & $0.00 \% \pm 0.00 \%$ & 0 & $0.00 \% \pm 0.00 \%$ \\
\hline
\end{tabular}




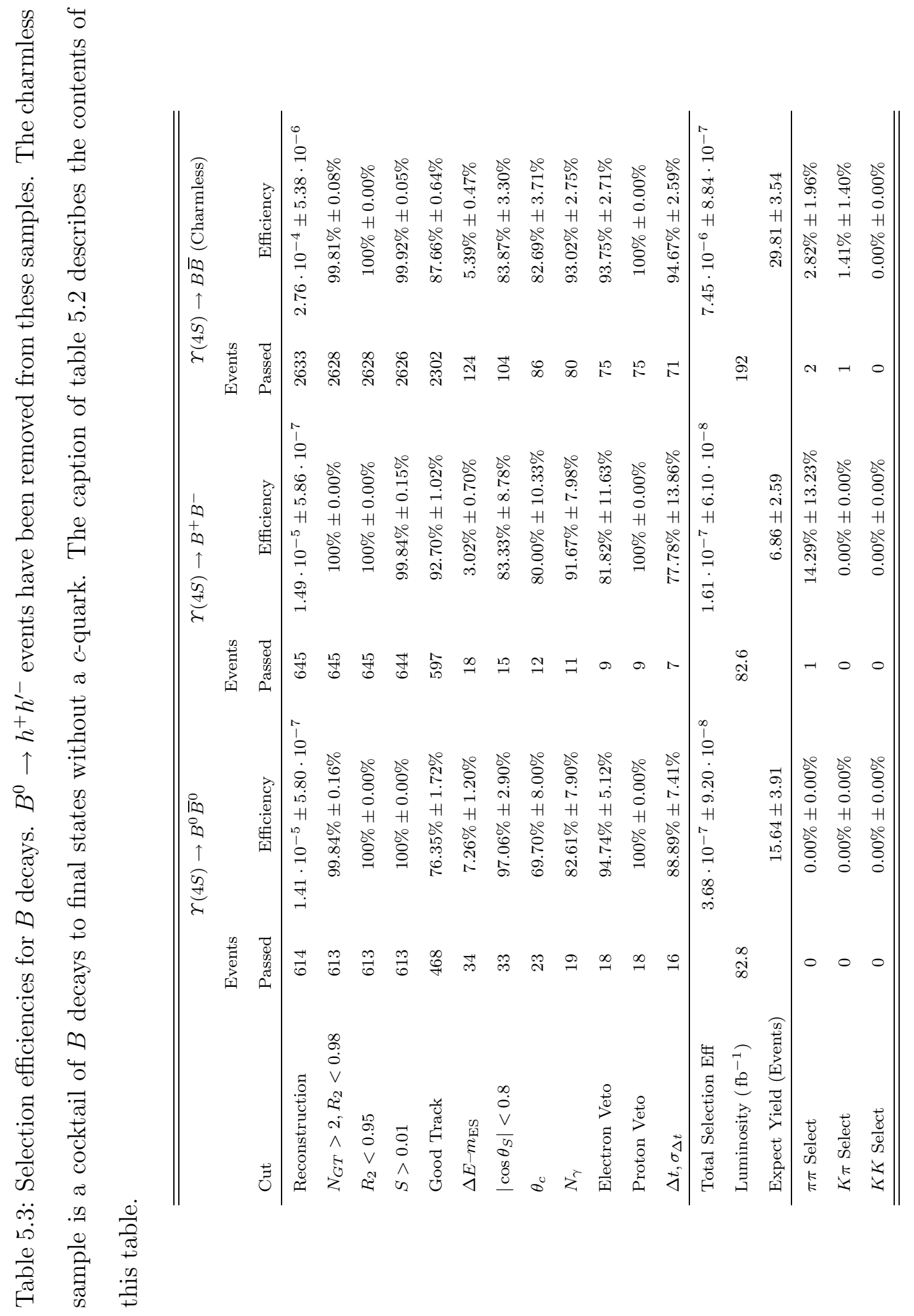




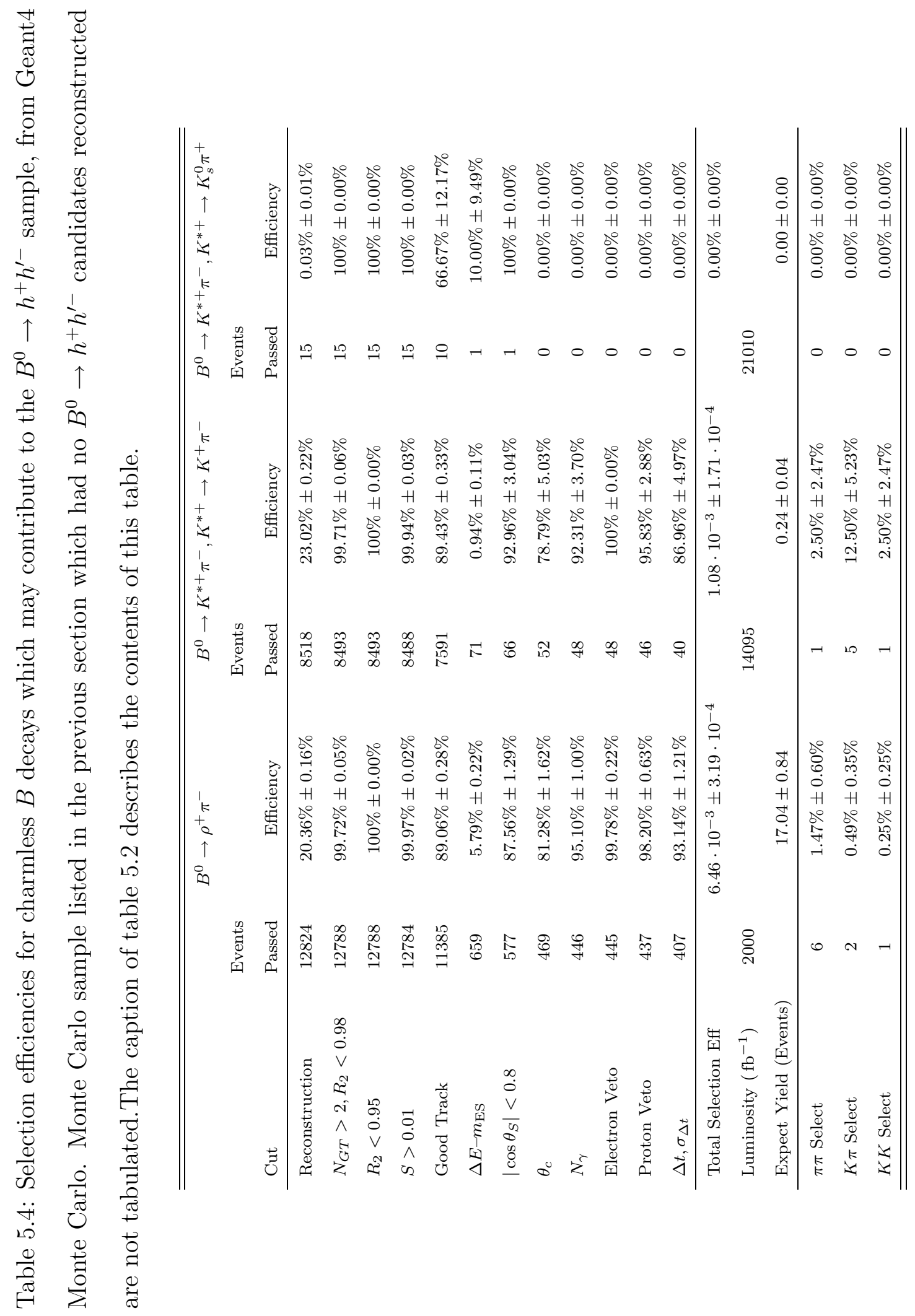




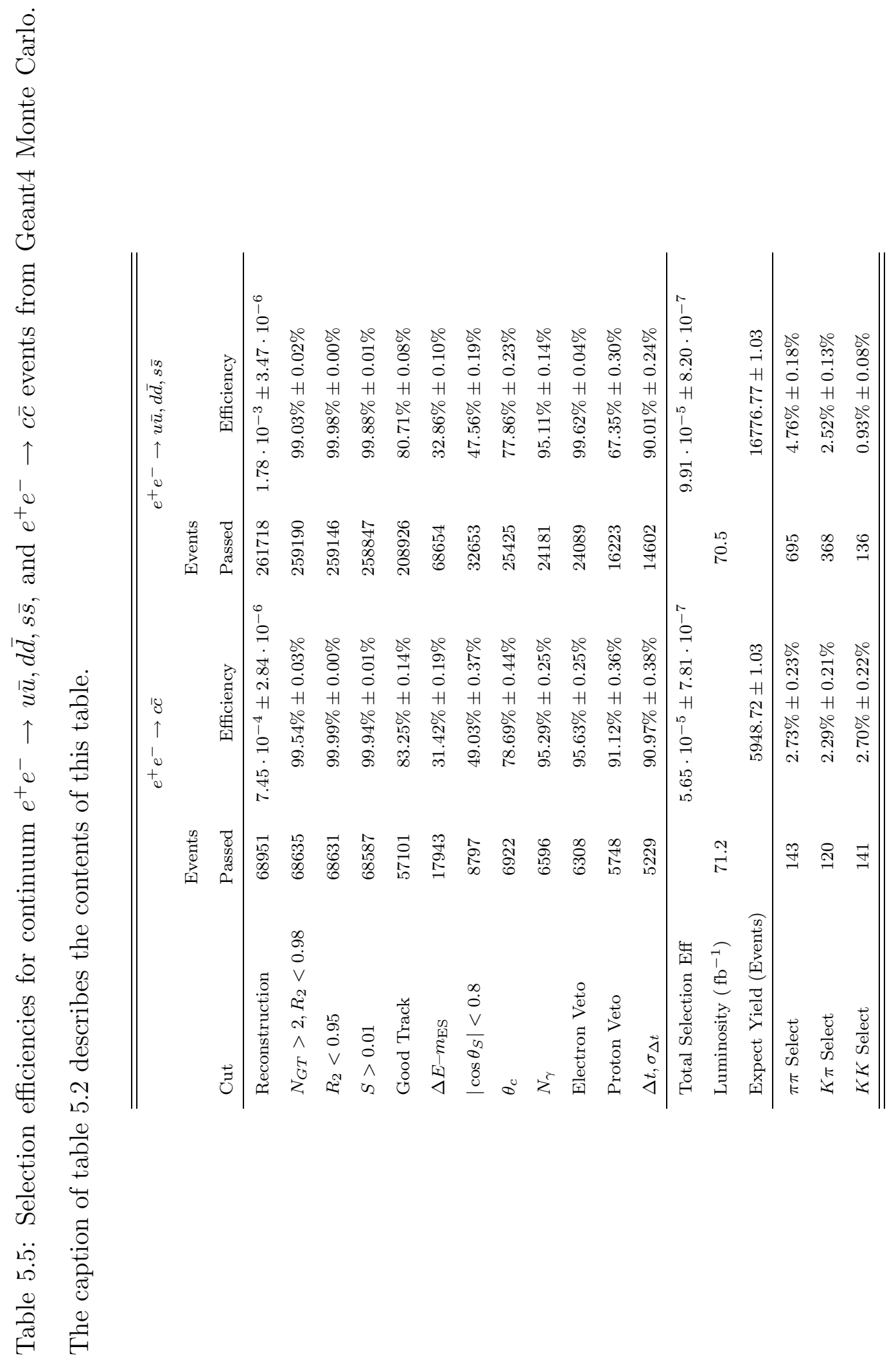




\section{Chapter 6}

\section{Measurement of the Branching Fractions}

Though by 1999 both $B^{0} \rightarrow \pi^{+} \pi^{-}$and $B^{0} \rightarrow K^{+} \pi^{-}$decays had been discovered by the CLEO collaboration [56], the sensitivity of theoretical hadronic calculations to the branching fractions of these and other two-body charmless decays warranted more precise measurements [15]. In addition, the prospect of discovering direct CP violation by observing a time-independent decay rate asymmetry between the selftagging $B^{0} \rightarrow K^{+} \pi^{-}$and $\bar{B}^{0} \rightarrow K^{-} \pi^{+}$modes has fueled a continuing interest in the refined measurements made possible by the accumulating data. With the present data sample of more than 80 million $B$ decays, the extraction of the branching fraction of the $B^{0} \rightarrow K^{+} \pi^{-}$decay, which is roughly four times larger and suffers from less background than $B^{0} \rightarrow \pi^{+} \pi^{-}$, is expected to yield $\approx 5 \%$ uncertainty and therefore may be considered a percision measurement. Hence greater understanding of the performance and behavior of analysis elements is now necessary. Having discussed the general analysis methods, we turn our attention in this chapter to the determination of analysis inputs, testing of analysis assumptions, the extraction of the results, and the evaluation of the systematic errors for the three branching fractions and the timeindependent $\mathrm{CP}$ asymmetry. 


\subsection{Maximum Likelihood fit for extracting Branch- ing Fractions}

Having selected a sample of events for fitting, the construction of the maximum likelihood fit may be itemized into the following tasks:

- the recognition of the origins of events which populate the sample,

- the selection of a set of uncorrelated variables which allow the separation of events into sub-samples,

- the identification of these sub-samples,

- the parameterization of the distributions of every variable for each sub-sample, and

- the identification of parameters which may be extracted from the sample.

In the previous chapter we noted that in addition to the signal $B$ decays, candidates mimicking $B^{0} \rightarrow h^{+} h^{--}$decays may originate from other $\Upsilon(4 s)$ decays, the hadronic $e^{+} e^{-} \rightarrow u \bar{u}, d \bar{d}, s \bar{s}, c \bar{c}$ continuum, QED $e^{+} e^{-} \rightarrow e^{+} e^{-}, \mu^{+} \mu^{-}, \tau^{+} \tau^{-}$processes, or beam backgrounds. Our selections virtually eliminated all background sources with the exception of the continuum events. In order to separate the signal from this background, we choose the kinematic quantities $m_{E S}$ and $\Delta E$, and the event shape Fisher discriminant $\mathcal{F}$ as fit variables. We also selected each track's $\theta_{c}$ measurement to identify the different final states. This choice of variables permit separating the sample into the eight "species" which we label by the flavor of the particles $\left(\pi^{+} \pi^{-}, K^{+} \pi^{-}\right.$, $\pi^{+} K^{-}$, or $K^{+} K^{-}$) and their source (signal or background). 
Table 6.1: Summary of PDFs and their parameters in the maximum likelihood fit. Parameters which are determined by the fit (ie floating) have no value or source specified. The indices' $k$ indicate species.

\begin{tabular}{ccccc}
\hline \hline Variable & Sig PDF & Sig Params & Bkg PDF & Bkg Params \\
\hline$m_{\mathrm{ES}}$ & Gaussian & $\mu_{m_{\mathrm{ES}}}=5.280 \pm 0.0005 \mathrm{GeV} / c^{2}$, & ARGUS & $\xi$ \\
& & $\sigma_{m_{\mathrm{ES}}}=2.6 \pm 0.1 \mathrm{MeV} / c^{2}$ & Function & \\
& & $\mu_{\Delta E}=-2_{-2}^{+1} \mathrm{MeV}$, & quadratic & $\Delta E_{p 1}, \Delta E_{p 2}$ \\
$\Delta E$ & Gaussian & $\sigma_{\Delta E}=26_{-0.7}^{+1.0} \mathrm{MeV}$ & \\
$\mathcal{F}$ & bifurcated & $\mu_{\mathcal{F}}=0.00929 \pm 0.01094$, & double & $\mathcal{F}_{\mu_{1}}, \mathcal{F}_{\sigma_{1}}, \mathcal{F}_{\mu_{2}}$, \\
& Gaussian & $\sigma_{\mathcal{F}}^{L}=0.69909 \pm 0.00676$, & Gaussian & $\mathcal{F}_{\sigma_{2}}, \mathcal{F}_{f_{1}}$ \\
& & $\sigma_{\mathcal{F}}^{R}=0.39737 \pm 0.00699$ & & \\
$\theta_{c}$ & double & $\mu_{\pi, K}^{ \pm}(\cos \theta)$, & same as & same as \\
& Gaussian & $\sigma_{\pi, K}^{ \pm}(\cos \theta)$ & Signal & Signal \\
& & from $D^{*} \operatorname{sample}$ & & \\
\hline \hline
\end{tabular}

\subsubsection{Fit Inputs}

We now turn our attention to the parameterization of fit variables for each species. For the background events we will only determine which empirical functions adequately describe each variable and allow the ML fit to extract the parameters of these PDFs. As discussed in the previous chapter, the simultaneous extraction of these parameters along with the signal/background yields, which was made possible by the inclusion of large number of sideband events in our sample, insures the propagation of the 

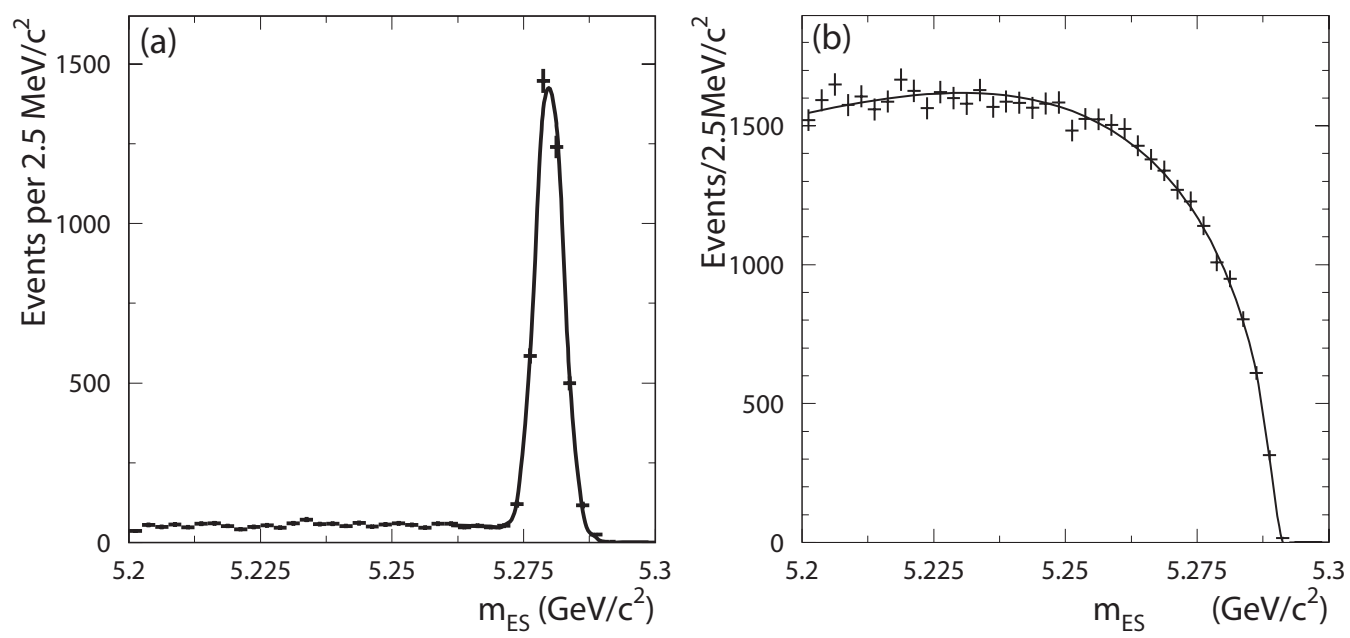

Figure 6.1: Sample $m_{\mathrm{ES}}$ distributions in data for (a) signal $B^{-} \rightarrow D^{0} \pi^{-}$decays and (b) continuum $B^{0} \rightarrow h^{+} h^{--}$candidates in the sideband region $|\Delta E|>.15 \mathrm{GeV}$. The $B^{-} \rightarrow D^{0} \pi^{-}$events are fitted to a Gaussian plus an ARGUS function to account for continuum backgrounds. The continuum sample is fitted to an ARGUS function.

statistical uncertainties on these parameters to those on our measured quantities. In contrast, we will determine the signal PDF functions and their parameters from control samples or Monte Carlo events which provide much larger statistics than the signal decays in the data. We will evaluate the systematic errors due to the signal PDF parameters by examining the change in the measured quantities when the sample is refitted with each parameter varied within its uncertainties. Table 6.1 summarizes the discussion of the PDFs and their parameters.

\section{Beam substituted mass, $m_{\mathrm{ES}}$}

As noted in chapter 4 , the reconstructed $m_{\mathrm{ES}}$ distribution of all $B$ decays into final states composed of only charged tracks is expected to be identical. Figure 6.1a illustrates a sample distribution for $B^{-} \rightarrow D^{0} \pi^{-}$decays, which Monte Carlo com- 
parisons indicate agree well with all $B^{0} \rightarrow h^{+} h^{\prime-}$ decays. From this sample we determine that signal $m_{\mathrm{ES}}$ decays are well represented by a Gaussian PDF with mean $\mu_{m_{\mathrm{ES}}}=5.280 \pm 0.0005 \mathrm{GeV} / c^{2}$ and resolution $\sigma_{m_{\mathrm{ES}}}=2.5 \pm 0.1 \mathrm{MeV} / c^{2}$.

No underlying physics governs fake candidates from the continuum. However, their $m_{E S}$ and $\Delta E$ distributions reflect the size of the available phase space and are therefore decay channel specific. The fall off of the $m_{\mathrm{ES}}$ distribution of the continuum sample at the beam energy, shown in figure $6.1 \mathrm{~b}$ for $B^{0} \rightarrow h^{+} h^{\prime-}$ candidates in the side-band region $|\Delta E|<.15 \mathrm{GeV}$, is well modeled by the one parameter ARGUS function [67] defined as:

$$
\frac{d N}{d m_{\mathrm{ES}}}=N \cdot m_{\mathrm{ES}} \cdot \sqrt{1-x^{2}} \cdot \exp \left(-\xi \cdot\left(1-x^{2}\right)\right)
$$

where $x=m_{\mathrm{ES}} / m_{\max }$ and $m_{\max }=5.2895 \mathrm{GeV} / c^{2}$ is largest $m_{\mathrm{ES}}$ value observed. Fits to sub-sets of this sideband sample where $\pi^{+} \pi^{-}, K^{ \pm} \pi^{\mp}$, or $K^{+} K^{-}$have been selected using particle identification information indicate no dependence on the particle content of the candidate and hence justify a common $\xi$ for all continuum background species. We allow this parameter, which is typically around 22 (see section 6.3), to be determined by the ML fit.

\section{The Energy Difference, $\Delta E$}

Monte Carlo studies (see figure 6.2a) suggests that $\Delta E$ distribution of $B^{0} \rightarrow \pi^{+} \pi^{-}$ events is well described by a single Gaussian function centered at $0 \mathrm{MeV}$ with a width of $25 \mathrm{MeV}$. As figure 6.3 a illustrates, the mean $\Delta E$ of $B^{0} \rightarrow K^{+} \pi^{-}$and $B^{0} \rightarrow K^{+} K^{-}$ decays is shifted by $\approx-42 \mathrm{MeV}$ and $\approx-95 \mathrm{MeV}$, when they are reconstructed as $B^{0} \rightarrow \pi^{+} \pi^{-}$. Also, since $\Delta E$ is calculated in the $\mathrm{CM}$ of the $B$ and is therefore sensitive to the boost, the incorrect mass hypotheses causes this shift to exhibit the momentum dependence displayed in figure $6.3 \mathrm{~b}$. We account for this feature in the 

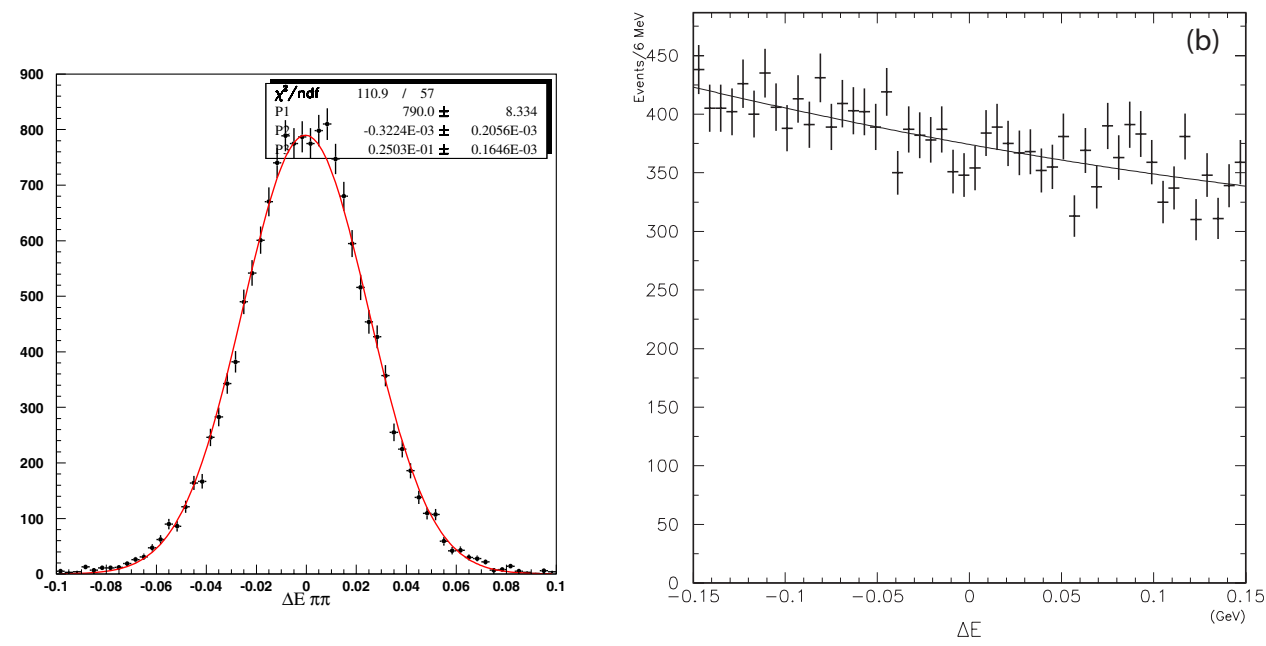

Figure 6.2: Distribution of $\Delta E$ for (a) signal $B^{0} \rightarrow \pi^{+} \pi^{-}$decays in Geant4 simulated events and (b) background continuum events from the sideband $m_{\mathrm{ES}}<5.26 \mathrm{GeV} / c^{2}$. The signal is fit with a Gaussian and the background is fit with a quadratic.
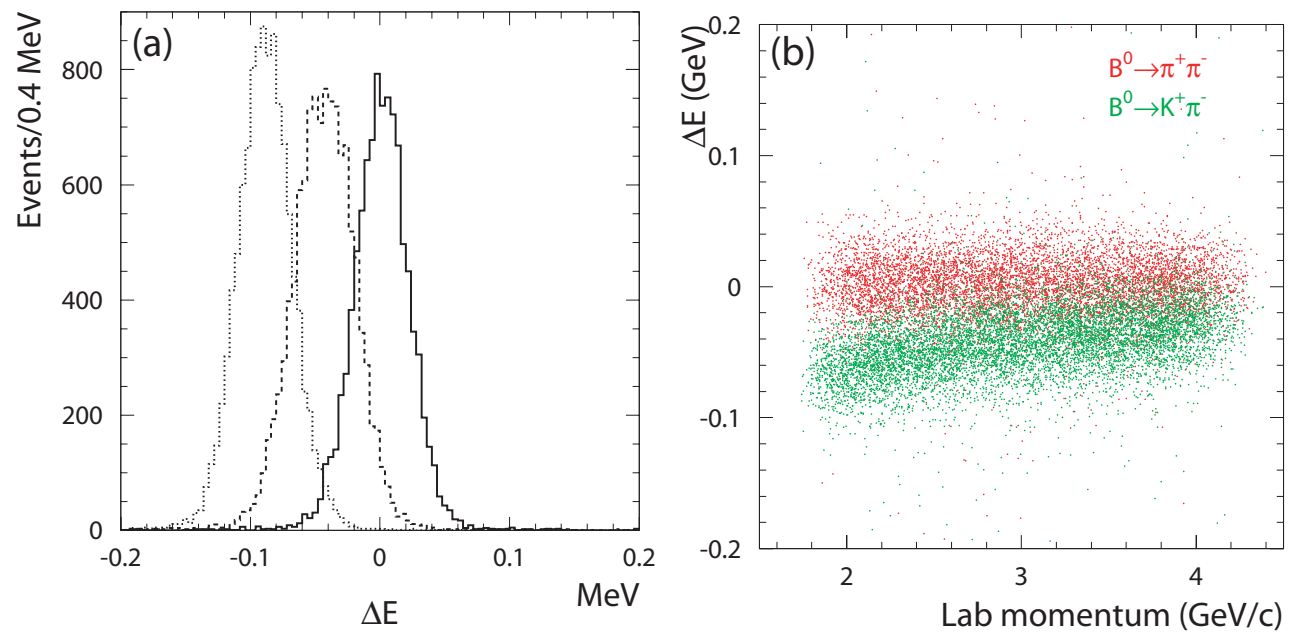

Figure 6.3: (a) Simulated $\Delta E$ distributions for (from right to left) $B^{0} \rightarrow \pi^{+} \pi^{-}$, $B^{0} \rightarrow K^{+} \pi^{-}$, and $B^{0} \rightarrow K^{+} K^{-}$decays reconstructed assuming both final state tracks are pions (a). (b) $\Delta E$ of simulated $B^{0} \rightarrow K^{+} \pi^{-}$(green) and $B^{0} \rightarrow \pi^{+} \pi^{-}$(red) events versus the momentum of one of the final state tracks. 
Table 6.2: Comparison of data and Monte Carlo $\Delta E$ resolution in $B^{-} \rightarrow D^{0} \pi^{-} \rightarrow$ $\left(K^{-} \pi^{+}\right) \pi^{-}$decays for different DCH high voltages. The fits to the data are displayed in figure 6.4 .

\begin{tabular}{lcc}
\hline \hline DCH High Voltage & Data $\sigma_{\Delta E}(\mathrm{MeV})$ & $\mathrm{MC} \sigma_{\Delta E}(\mathrm{MeV})$ \\
\hline $1900 \mathrm{~V}$ & $18.1 \pm 0.7$ & $16.0 \pm 0.3$ \\
$1960 \mathrm{~V}$ & $16.9 \pm 0.7$ & $16.1 \pm 0.2$ \\
$1930 \mathrm{~V}$ & $16.5 \pm 0.3$ & $16.0 \pm 0.1$ \\
\hline Average & $16.8 \pm 0.3$ & $16.0 \pm 0.1$ \\
Scale Factor & $1.05 \pm 0.02$ & \\
\hline \hline
\end{tabular}

$\Delta E$ distribution of $B^{0} \rightarrow K^{+} \pi^{-}$by employing the analytical expression of the shift

$$
\mu_{\Delta E}(K \pi)=-\gamma\left[\sqrt{m_{K}^{2}+p^{2}}-\sqrt{m_{\pi}^{2}+p^{2}}\right],
$$

for the mean of the $\mathrm{PDF}$ which represents its $\Delta E$ distribution. An analogous relation provides the shift for $B^{0} \rightarrow K^{+} K^{-}$.

Since the kinematics of the tracks from the $B^{0} \rightarrow h^{+} h^{\prime-}$ decays dictate a unique $\Delta E$ distribution for the signal modes (see section 5.1.1) we cannot simply rely on the reconstruction of more abundant $B$ decays to assess the $\Delta E$ resolution in data. Also, comparisons of the data and Geant4 simulated events (see below) suggest that the Monte Carlo simulation generally under-estimates the resolution and does not accurately reproduce an observed negative shift in the mean of $\Delta E$. In light of these facts, we choose to estimate the parameters of the signal $\Delta E \mathrm{PDF}$ by correcting $B^{0} \rightarrow h^{+} h^{-}$Monte Carlo with the measured discrepancy of simulated events with data in more abundant modes. Ultimately, we find our estimates are consistent with the mean and resolution determined directly from the fit to the $B^{0} \rightarrow h^{+} h^{-}$ 
Table 6.3: Comparison of data and Monte Carlo $\Delta E$ mean in $B^{-} \rightarrow D^{0} \pi^{-} \rightarrow$ $\left(K^{-} \pi^{+}\right) \pi^{-}$decays for difference DCH high voltages. The fits to the data are displayed in figure 6.4 .

\begin{tabular}{ccc}
\hline \hline Sample & Data $\mu_{\Delta E}(\mathrm{MeV})$ & $\mathrm{MC} \mu_{\Delta E}(\mathrm{MeV})$ \\
\hline $1900 \mathrm{~V}$ & $-4.8 \pm 0.8$ & $0.1 \pm 0.3$ \\
$1960 \mathrm{~V}$ & $-4.8 \pm 0.8$ & $-0.4 \pm 0.2$ \\
$1930 \mathrm{~V}$ & $-3.1 \pm 0.5$ & $-0.3 \pm 0.1$ \\
\hline \hline
\end{tabular}

candidates (see section 6.3.3).

Our understanding of $\Delta E$ is based on several key observations, predominately from studies of $B^{-} \rightarrow D^{0} \pi^{-} \rightarrow\left(K^{-} \pi^{+}\right) \pi^{-}$decays, which approximate the track momenta of the $B^{0} \rightarrow h^{+} h^{\prime-}$ final states better than any other $B$ decay with a large branching fraction. From the comparisons of data and Monte Carlo presented in tables 6.2 and 6.3 , we conclude:

- Both data and Monte Carlo suggest that neither the mean nor resolution of $\Delta E$ exhibit a clear dependence on the DCH high voltage. Such a dependence could have been caused by correlations between the high voltage and the average number of hits on tracks.

- As figure 6.4 illustrate, $\Delta E$ for $B$ decays are well represented by a single Gaussian function.

- The Monte Carlo underestimates the $\Delta E$ resolution by a factor of $1.05 \pm .02$.

- The mean of $\Delta E$ in data is shifted negatively. 

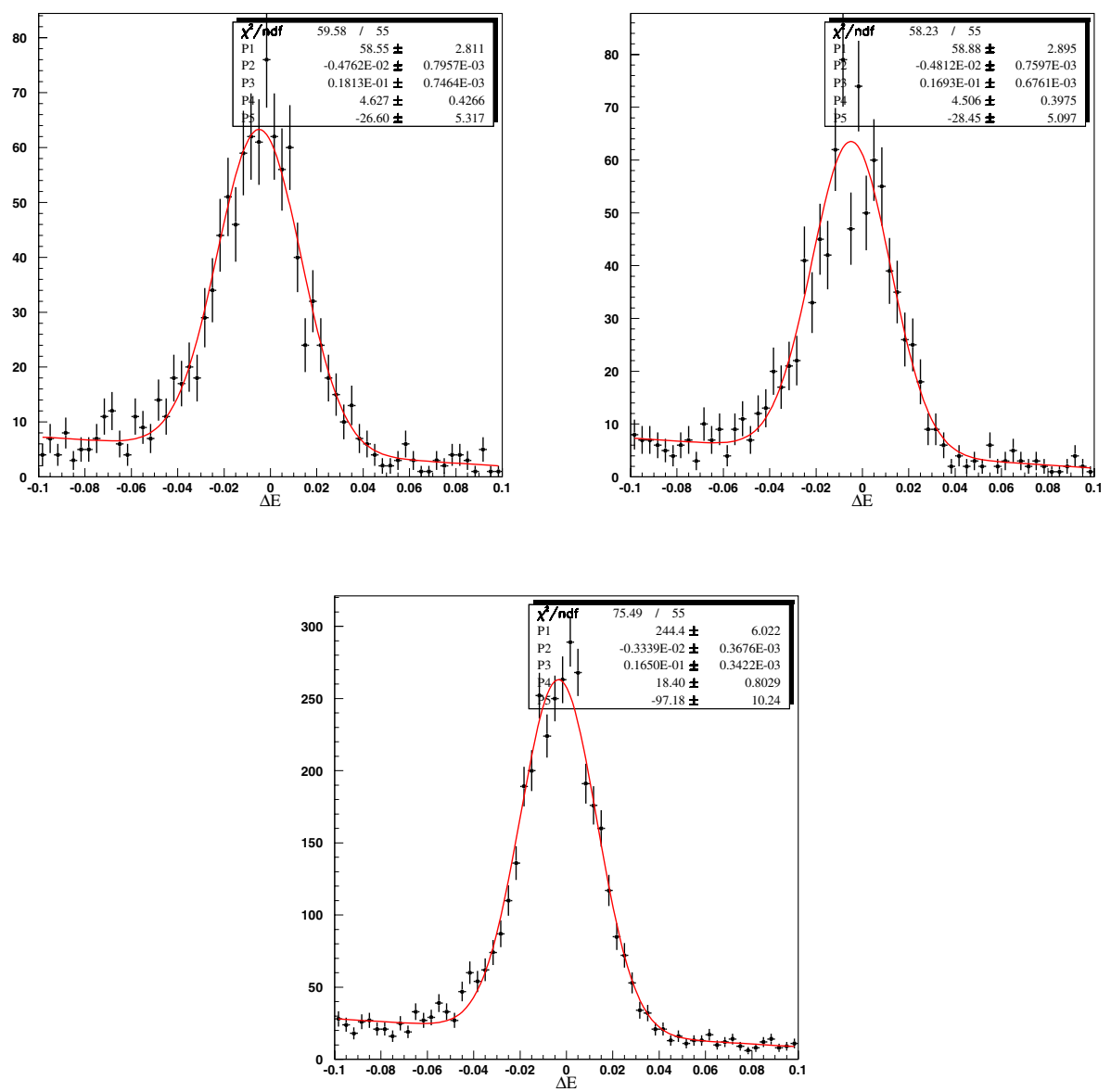

Figure 6.4: Distribution of $\Delta E$ for $B^{-} \rightarrow D^{0} \pi^{-} \rightarrow\left(K^{-} \pi^{+}\right) \pi^{-}$reconstructed events in data taken with DCH voltage at (a) $1960 \mathrm{~V}$, (b) $1900 \mathrm{~V}$, and (c) $1930 \mathrm{~V}$. The fits are to a Gaussian with a polynomial background component. 
We scale the Monte Carlo $B^{0} \rightarrow h^{+} h^{\prime-} \Delta E$ resolution of $25 \mathrm{MeV}$ by the observed data/Monte Carlo discrepancy in $B^{-} \rightarrow D^{0} \pi^{-}$decays. This procedure leads to $\sigma_{\Delta E}=26_{-0.7}^{+1.0} \mathrm{MeV}$ where the upper error has been conservatively increased and the lower error is unchanged since the resolution in data is not expected to be smaller than in the simulation.

Almost all samples of fully reconstructed $B$ decays at $B A B A R$ demonstrate a negative $\Delta E$ shift, which the Geant4 simulation confirms in direction but under-estimates in magnitude. This bias is most likely the consequence of a combination of three effects [68]:

1. insufficient material employed in the calculation of the energy loss of charged tracks in detector (specifically the $\mathrm{DCH}$ ),

2. uncertainty in the strength of the magnetic field, or

3. the assumption that all tracks are pions in the tracking algorithms.

The magnitude of the bias generally depends on particles contributing to the final state. Therefore despite the apparent large shift of $>4 \mathrm{MeV}$ in $B^{-} \rightarrow D^{0} \pi^{-}$decays, most other modes present smaller shifts. For example, $B^{0} \rightarrow J / \psi K^{+}$decays exhibit only a small shift of $-1.7 \pm 0.2 \mathrm{MeV}$. Some studies indicate that the shift in $B^{-} \rightarrow$ $D^{0} \pi^{-}$may be due to the presence of a low momentum kaon in the final state which would be most significantly affected by (3). Considering that the Geant4 simulation demonstrate no shift for the $B^{0} \rightarrow h^{+} h^{\prime-}$ decays, we assign $\mu_{\Delta E}=-2_{-2}^{+1} \mathrm{MeV}$ for our signal decays. This choice includes the possibility of a small shift, while allowing the values as large as that of $B^{-} \rightarrow D^{0} \pi^{-}$decays.

Figure $6.2 \mathrm{~b}$ displays $\Delta E$ distribution for continuum $B^{0} \rightarrow h^{+} h^{\prime-}$ candidates, obtained in the sideband region $m_{\mathrm{ES}}<5.26 \mathrm{GeV} / c^{2}$. This distribution is well described 
by a second order polynomial. As with $m_{\mathrm{ES}}$, the continuum $\Delta E$ exhibits no significant dependence on the particles comprising the candidates. Therefore we choose to extract one set of parameters for all background species in the ML fit.

\section{The Fisher Discriminant, $\mathcal{F}$}

Figure 6.5 displays the signal and continuum background Fisher discriminant distributions obtained from $B^{0} \rightarrow \pi^{+} \pi^{-}$Monte Carlo and $B^{0} \rightarrow h^{+} h^{--}$candidates in the $m_{\mathrm{ES}}$ sideband. We parameterize the signal distribution with a bifurcated Gaussian (a Gaussian with a different width on the left and right sides of the mean) and the continuum with a double Gaussian (the sum of two Gaussians). Once again, we permit the ML fit to extract the parameters of the double Gaussian for the background. When evaluating systematics, we also consider a triple Gaussian which better describes the $\approx 0.05 \%$ of background events not well described by the the double Gaussian parameterization.

\section{The Cherenkov Angles, $\theta_{c}^{+}, \theta_{c}^{-}$}

The $D^{*}$ control sample described in 5.1 .3 provides the parameterizations of the polarangle dependence of the resolution and bias of the measured Cherenkov angle. We represent $\pi^{+}, \pi^{-}, K^{+}$, and $K^{-}$tracks by the different probability distribution functions of

$$
\left(\theta_{c}-\theta_{c}^{\operatorname{Exp}}-\mu_{\pi, K}^{ \pm}(\cos \theta)\right) / \sigma_{\pi, K}^{ \pm}(\cos \theta)
$$

displayed in figure 5.4 and assign each species the appropriate PDFs product for one positive and a negative particle. Since the measured resolutions and biases are considered to be a calibration, we derive the systematic errors due to $\theta_{c}$ from the statistical errors of the double Gaussian fits to the pull distributions in the control 

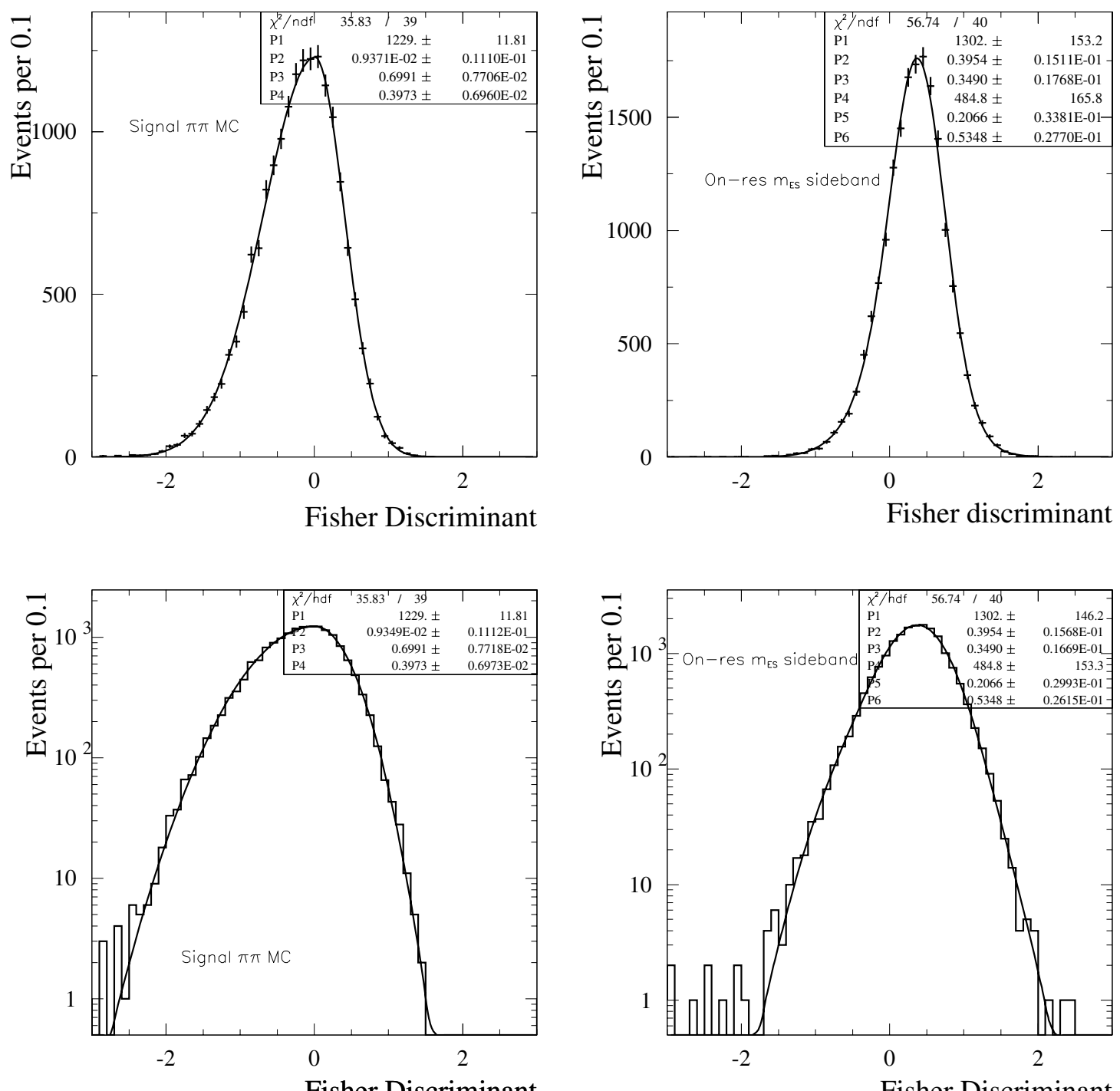

Figure 6.5: Distribution of $\mathcal{F}$ in signal $B^{0} \rightarrow \pi^{+} \pi^{-}$Monte Carlo (left) and onresonance $m_{\mathrm{ES}}<5.26 \mathrm{GeV} / c^{2}$ sideband data (right), on linear (top) and logarithmic (bottom) scales. The signal is fit to a bifurcated Gaussian and the background is to the sum of two Gaussians. 
samples. We evaluate these uncertainties by varying the fit parameters within their error and then reperforming the ML fit on the data. In order to account for the correlations between these parameters, we use the diagonalized correlation matrices of the double Gaussian fits to determine the eight variations magnitudes and their four independent directions.

\subsubsection{Correlations Between Variables}

In section 5.2.1 we relied on the assumption that the fit variables are uncorrelated in order to write total probability for each event as the product of individual variable PDFs. As a precaution, we check this assumption by explicitly calculating the linear correlations between pairs of input variables $\left\{x_{i}\right\}$, defined as

$$
c_{i j}=s_{i j}^{2} / s_{i i} s_{j j}
$$

where

$$
s_{i j}=\frac{1}{N-1} \sum_{k}^{\text {Events }}\left(x_{i}^{k}-\bar{x}_{i}\right)\left(x_{j}^{k}-\bar{x}_{j}\right) .
$$

These are tabulated for signal and background samples in table 6.4. The largest observed correlation is $\approx-13 \%$ between $m_{\mathrm{ES}}$ and $\Delta E$ for signal decays.

\subsubsection{Fit Parameters}

Table 6.5 summarizes the parameters of the ML fit. The fit extracts 16 parameters from the $B^{0} \rightarrow h^{+} h^{-}$sample, 12 of which describe the background. The four signal parameters are the asymmetry in the $K \pi$ final state and the yields, from which we may calculate the branching fractions. To simplify the interpretation of the $B^{0} \rightarrow K^{+} \pi^{-}$branching fraction and asymmetry, we reparameterize the

signal and background yields $N_{K^{+} \pi^{-}}^{S, B}$ and $N_{K^{-} \pi^{+}}^{S, B}$ as $N_{K \pi}^{S, B}=N_{K^{+} \pi^{-}}^{S, B}+N_{K^{-} \pi^{+}}^{S, B}$ and 
Table 6.4: Linear correlation coefficients for the variables $\left\{m_{\mathrm{ES}}, \Delta E, \mathcal{F}, \theta_{c}^{+}, \theta_{c}^{-}\right\}$calculated for events in the $5.2<m_{\mathrm{ES}}<5.26 \mathrm{GeV} / c^{2}$ sideband region and the $B^{0} \rightarrow \pi^{+} \pi^{-}$ Monte Carlo events.

\begin{tabular}{ccc}
\hline \hline Variables & Sideband & $\pi \pi \mathrm{MC}$ \\
\hline$\left(m_{\mathrm{ES}}, \Delta E\right)$ & 0.00426 & -0.12767 \\
$\left(m_{\mathrm{ES}}, \mathcal{F}\right)$ & 0.01350 & 0.00086 \\
$\left(m_{\mathrm{ES}}, \theta_{c}^{+}\right)$ & 0.00041 & 0.01223 \\
$\left(m_{\mathrm{ES}}, \theta_{c}^{-}\right)$ & -0.00536 & 0.00384 \\
$(\Delta E, \mathcal{F})$ & -0.03508 & 0.00317 \\
$\left(\Delta E, \theta_{c}^{+}\right)$ & 0.02527 & -0.00393 \\
$\left(\Delta E, \theta_{c}^{-}\right)$ & 0.01923 & 0.00967 \\
$\left(\mathcal{F}, \theta_{c}^{+}\right)$ & 0.00891 & -0.01662 \\
$\left(\mathcal{F}, \theta_{c}^{-}\right)$ & 0.00571 & -0.01425 \\
$\left(\theta_{c}^{+}, \theta_{c}^{-}\right)$ & 0.04335 & -0.04814 \\
\hline \hline
\end{tabular}


$\mathcal{A}_{K \pi}^{B}=N_{K^{-} \pi^{+}}^{S, B}-N_{K^{+} \pi^{-}}^{S, B} / N_{K^{-} \pi^{+}}^{S, B}+N_{K^{+} \pi^{-}}^{S, B}$. Also since the $B^{0} \rightarrow K^{+} K^{-}$yield is expected to be small, we permit Minuit to explore negative values of $N_{K K}^{S}$ in order to obtain proper calculation of the derivatives of the likelihood with respect to this parameter.

\subsection{Fit Validations}

The maximum likelihood fit that we have constructed is essentially a black box which when provided with a set of measured quantities for a sample of events, statistically extracts some measurements from the whole dataset. This fit's complexity and opaqueness necessitates scrutiny of its performance. The required testing is especially important since, in an attempt to minimize biases which we may propagate to the measurements, we have choosen to blind ourselves to the direct influence of our analysis decisions on the results by avoiding using the final data-set while determining our selections and developing the fit. Ideally, once the analysis technique is fully defined and reviewed, we freeze it and fit the data without the possibility of change. The discovery and correction of a mistake after "unblinding" may compromise our objectivity, so we must be confident that the analysis is sound before fitting the data.

\subsubsection{Toy Monte Carlo Tests}

Fits to large samples of toy Monte Carlo experiments test the ML fit's ability to properly measure parameters which we input. Since our toy Monte Carlo reproduces features of the data which have not been explicitly built into the ML fit (such as the kinematics of the decays and their correlations with the DIRC), these studies also test some of the fit's assumptions about the dataset. We produce toy Monte Carlo 
Table 6.5: Summary of parameters in the branching fraction maximum likelihood fit.

\begin{tabular}{|c|c|c|}
\hline Parameter & Status & Description \\
\hline$N_{\pi \pi}^{S}$ & float & number of signal $\pi \pi$ events \\
\hline$N_{K \pi}^{S}$ & float & number of signal $K \pi$ events \\
\hline$N_{K K}^{S}$ & float & number of signal $K K$ events \\
\hline $\mathcal{A}_{K \pi}^{S}$ & float & charge asymmetry in signal $K^{ \pm} \pi^{\mp}$ events \\
\hline$N_{\pi \pi}^{B}$ & float & number of background $\pi \pi$ events \\
\hline$N_{K \pi}^{B}$ & float & number of background $K \pi$ events \\
\hline$N_{K K}^{B}$ & float & number of background $K K$ events \\
\hline $\mathcal{A}_{K \pi}^{B}$ & float & charge asymmetry in background $K^{ \pm} \pi^{\mp}$ events \\
\hline$\mu_{m} \mathrm{ES}$ & fixed & signal $m_{\mathrm{ES}}$ mean \\
\hline$\sigma m_{\mathrm{ES}}$ & fixed & signal $m_{\mathrm{ES}}$ resolution \\
\hline$\xi$ & float & background $m_{\mathrm{ES}}$ ARGUS shape parameter \\
\hline$\mu_{\Delta E}$ & fixed & signal $\Delta E$ mean \\
\hline$\sigma_{\Delta E}$ & fixed & signal $\Delta E$ resolution \\
\hline$\Delta E_{p 1}$ & float & background $\Delta E$ linear term \\
\hline$\Delta E_{p 2}$ & float & background $\Delta E$ quadratic term \\
\hline$\mu_{\mathcal{F}}$ & fixed & signal $\mathcal{F}$ mean \\
\hline$\sigma_{\mathcal{F}}^{L}$ & fixed & signal $\mathcal{F}$ left width \\
\hline$\sigma_{\mathcal{F}}^{R}$ & fixed & signal $\mathcal{F}$ right width \\
\hline $\mathcal{F}_{f_{1}}$ & float & background Fisher fraction of first Gaussian \\
\hline $\mathcal{F}_{\mu_{1}}$ & float & background Fisher mean of first Gaussian \\
\hline $\mathcal{F}_{\sigma_{1}}$ & float & background Fisher width of first Gaussian \\
\hline $\mathcal{F}_{\mu_{2}}$ & float & background Fisher mean of second Gaussian \\
\hline $\mathcal{F}_{\sigma_{2}}$ & float & background Fisher width of second Gaussian \\
\hline
\end{tabular}



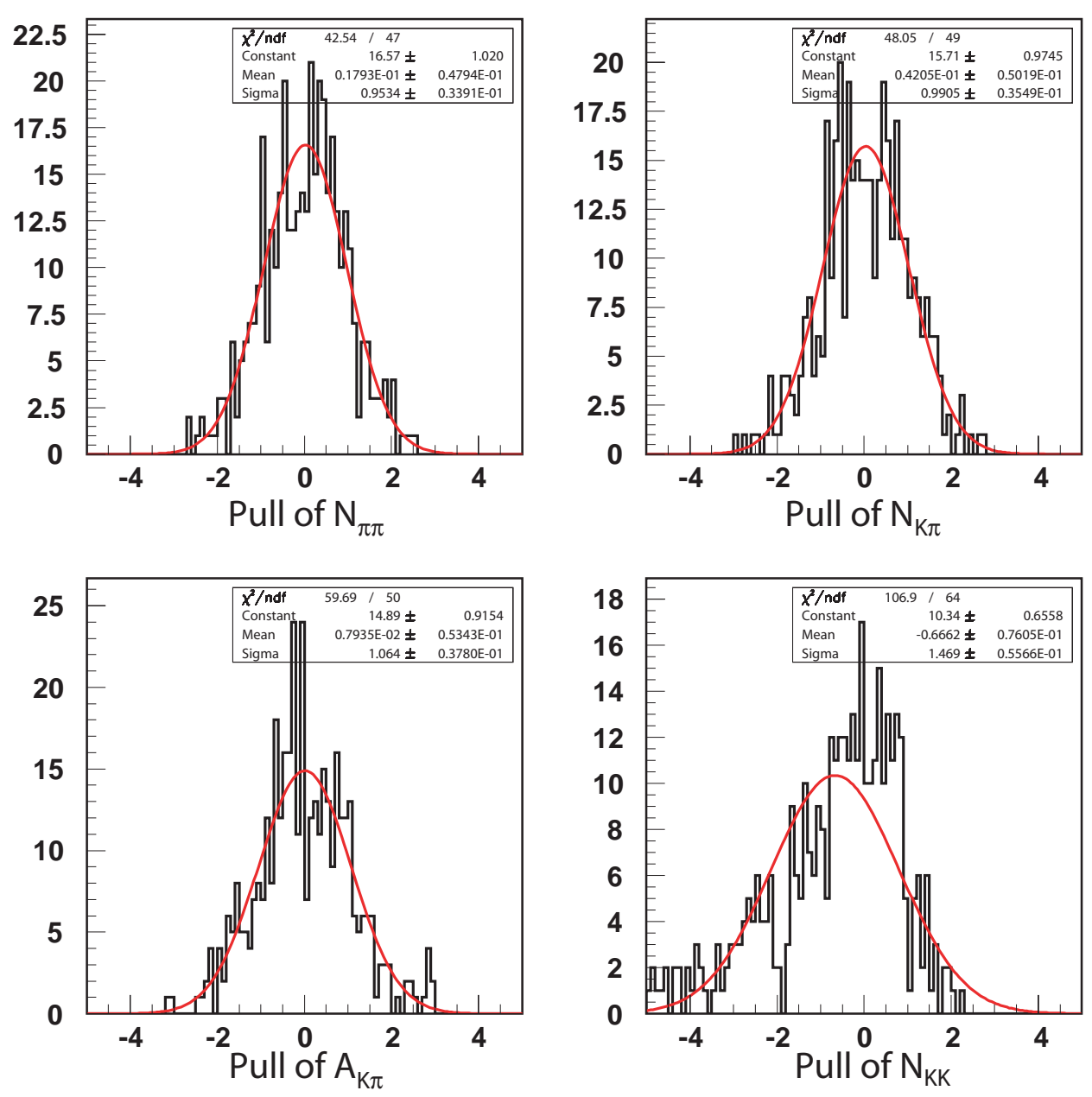

Figure 6.6: The distribution of the pulls of $N_{\pi \pi}^{S}, N_{K \pi}^{S}, \mathcal{A}_{K \pi}^{S}$, and $N_{K K}^{S}$ from fits to toy Monte Carlo experiments, fitted to Gaussian functions. 
experiments which simulate the expected data sample by using the signal parameters (discussed in the section 6.1.1) and yields and background parameters from our earlier measurements of these modes based on smaller data-sets. In each experiment, we use Poisson distributions with means at the expected yields for the full data set to select how many events of each species to generate. Since $B^{0} \rightarrow K^{+} K^{-}$decays have not been observed, we generate no events for this species.

Figure 6.6 displays pull distributions for the four signal yield parameters derived from fits to an ensemble of such experiments. In each experiment, the pull for a parameter $x$ is defined as $\left(x_{\mathrm{Fit}}-x_{\mathrm{In}}\right) / \sigma_{x_{\mathrm{Fit}}}$, where $x_{\mathrm{Fit}}$ and $\sigma_{x_{\mathrm{Fit}}}$ are the fitted value and error, and $x_{\text {In }}$ is the inputed value. We deduce two important observations from the mean and width of these distributions: the fit exhibits no large biases and the fitted errors correctly estimate the spread in the distributions of fitted values. The behavior of the $N_{K K}^{S}$ pull is the typical consequence of negative background fluctuations when there is no signal in the sample. Since we calculate the upper limit

on the $B^{0} \rightarrow K^{+} K^{-}$branching fraction by integrating the likelihood (see section 6.3 ), we ignore the fitted value of $N_{K K}^{S}$.

\subsubsection{Geant4 Monte Carlo Tests}

Although limited by the available background Monte Carlo statistics, Geant4 simulated events reproduce correlations between the variables which the toy Monte Carlo ignores. Hence, despite the disagreements between variable distributions from simulated events and data, these samples are an important complement to the toy $\mathrm{MC}$ tests. For consistency we use parameterizations of PDF parameters derived from the simulated events. For example we repeat the $\theta_{c}$ study using $D^{*}$ Monte Carlo.

As table 6.6 indicates, fits to large signal samples display a very small $\pi \leftrightarrow K$ 
Table 6.6: Summary of test fits on signal Monte Carlo samples of 50,000 $B^{0} \rightarrow$ $\pi^{+} \pi^{-}$and 200,000 $B^{0} \rightarrow K^{+} \pi^{-}$events. The choice of number of events roughly approximates the ratio of the branching fractions.

\begin{tabular}{cccc}
\hline \hline & & & $200 k B^{0} \rightarrow K^{+} \pi^{-}$ \\
Parameter & $50 k B^{0} \rightarrow \pi^{+} \pi^{-}$ & $200 k B^{0} \rightarrow K^{+} \pi^{-}$ & $50 k B^{0} \rightarrow \pi^{+} \pi^{-}$ \\
\hline$N_{\pi \pi}^{S}$ & $49722 \pm 223$ & $63.3 \pm 19.7$ & $50688 \pm 238$ \\
$N_{K \pi}^{S}$ & $114 \pm 14$ & $199560 \pm 447$ & $198860 \pm 453$ \\
$N_{K K}^{S}$ & $0.5 \pm 1.2$ & $2.7 \pm 8.9$ & $2.0 \pm 8.5$ \\
$\mathcal{A}_{K \pi}^{S}$ & $0.15 \pm 0.12$ & $-0.004 \pm 0.002$ & $-0.001 \pm 0.002$ \\
$N_{\pi \pi}^{B}$ & $158 \pm 15$ & $65.6 \pm 10.1$ & $179 \pm 17$ \\
$N_{K \pi}^{B}$ & $6.1 \pm 3.9$ & $303 \pm 21$ & $280 \pm 21$ \\
$N_{K K}^{B}$ & $0.0 \pm 0.7$ & $2.1 \pm 3.6$ & $2.0 \pm 3.7$ \\
$\mathcal{A}_{K \pi}^{B}$ & $-1.0 \pm 1.5$ & $0.059 \pm 0.068$ & $0.075 \pm 0.072$ \\
\hline \hline
\end{tabular}

$(<<1 \%)$ cross-feed and negligible signal to background migration. Also the results of a fit to the available simulated background sample, shown in table 6.7 , provides confidence that the background events are unlikely to be misidentified as signal $(\approx 1$ in 10000 background to signal feed through). This table also illustrates that the fit performs as expected when signal events are mixed with the background sample.

\subsubsection{Test Fits on Data}

The evaluation of the fit on recorded data is limited to fits to the small off resonance subsample of background events, where no signal events are expected. Table 6.8 summarizes the result. We observe no signal events and find that the background 
Table 6.7: Summary of test fits on the continuum Monte Carlo samples, with and without appropriate number of signal events mixed in.

\begin{tabular}{ccc}
\hline \hline & & $150 B^{0} \rightarrow \pi^{+} \pi^{-}+500 B^{0} \rightarrow K^{+} \pi^{-}+$ \\
Parameter & 20274 continuum & 20274 continuum \\
\hline$N_{\pi \pi}^{S}$ & $0 \pm 1.4$ & $149 \pm 17$ \\
$N_{K \pi}^{S}$ & $2.4 \pm 3.8$ & $497 \pm 25$ \\
$N_{K K}^{S}$ & $-4.9 \pm 5.7$ & $-2.5 \pm 6.4$ \\
$\mathcal{A}_{K \pi}^{S}$ & $1.0 \pm 1.9$ & $-0.008 \pm 0.051$ \\
$N_{\pi \pi}^{B}$ & $10699 \pm 105$ & $10721 \pm 106$ \\
$N_{K \pi}^{B}$ & $5666 \pm 79$ & $5657 \pm 80$ \\
$N_{K K}^{B}$ & $3912 \pm 64$ & $3909 \pm 64$ \\
$\mathcal{A}_{K \pi}^{B}$ & $-0.015 \pm 0.014$ & $-0.016 \pm 0.014$ \\
\hline \hline
\end{tabular}


Table 6.8: Summary of ML fit on the off-resonance data sample.

\begin{tabular}{cc}
\hline \hline Parameter & Fitted Value \\
\hline$N_{\pi \pi}^{S}$ & $0.0 \pm 6.3$ \\
$N_{K \pi}^{S}$ & $2.7 \pm 2.7$ \\
$N_{K K}^{S}$ & $-7.2 \pm 2.3$ \\
$\mathcal{A}_{K \pi}^{S}$ & $0.5 \pm 1.5$ \\
$N_{\pi \pi}^{B}$ & $1451 \pm 40$ \\
$N_{K \pi}^{B}$ & $984 \pm 35$ \\
$N_{K K}^{B}$ & $696 \pm 28$ \\
$\mathcal{A}_{K \pi}^{B}$ & $0.064 \pm 0.035$ \\
\hline \hline
\end{tabular}

species composition agrees with expectation from the sidebands. Similarly, we find that all of the fitted background parameters are also consistent with expected values.

\subsection{Results}

The results of the ML fit to the full on-resonance data sample is displayed in table 6.9. We calculate the branching fractions from the yields using

$$
\mathcal{B}=\frac{N}{\epsilon N_{B \bar{B}}},
$$

where $N$ is the measured yield, $\epsilon$ is the efficiency (from table 5.1), and $N_{B \bar{B}}=$ $87.9 \pm 1.0$ million is the measured number of $B \bar{B}$ events in the on-resonance sample (see section 4.3.2). We find $\mathcal{B}\left(B^{0} \rightarrow \pi^{+} \pi^{-}\right)=(156.5 \pm 18.9) \times 10^{-6}, \mathcal{B}\left(B^{0} \rightarrow\right.$ $\left.K^{+} \pi^{-}\right)=(588.5 \pm 29.6) \times 10^{-6}$, and $\mathcal{B}\left(B^{0} \rightarrow K^{+} K^{-}\right)=(0.8 \pm 7.7) \times 10^{-6}$, where the errors are statistical only. We calculate the $90 \%$ confidence upper limit on 
Table 6.9: The values of parameters from the branching fraction fit to the full onresonance dataset.

\begin{tabular}{cc}
\hline \hline Parameter & BR Fit \\
\hline$N_{\pi \pi}^{S}$ & $156.5 \pm 18.9$ \\
$N_{K \pi}^{S}$ & $588.5 \pm 29.6$ \\
$\mathcal{A}_{K \pi}^{S}$ & $-0.102 \pm 0.050$ \\
$N_{K K}^{S}$ & $0.8 \pm 7.7$ \\
$N_{\pi \pi}^{B}$ & $12200 \pm 117$ \\
$N_{K \pi}^{B}$ & $7984 \pm 102$ \\
$\mathcal{A}_{K \pi}^{B}$ & $0.014 \pm 0.013$ \\
$N_{K K}^{B}$ & $5155 \pm 77$ \\
$\xi$ & $22.0 \pm 0.7$ \\
$\Delta E_{p 1}$ & $-0.749 \pm 0.074$ \\
$\Delta E_{p 2}$ & $0.683 \pm 0.952$ \\
$\mathcal{F}_{f_{1}}$ & $0.846 \pm 0.025$ \\
$\mathcal{F}_{\mu_{1}}$ & $0.367 \pm 0.005$ \\
$\mathcal{F}_{\sigma_{1}}$ & $0.380 \pm 0.006$ \\
$\mathcal{F}_{\mu_{2}}$ & $0.104 \pm 0.030$ \\
$\mathcal{F}_{\sigma_{2}}$ & $0.640 \pm 0.020$ \\
\hline \hline &
\end{tabular}


$\mathcal{B}\left(B^{0} \rightarrow K^{+} K^{-}\right)=15.9 \times 10^{-6}$ by determining the point $N_{K K}^{9}$ where the likelihood $L_{\max }\left(N_{K K}\right)$, maximized with respect to all free fit parameters while $N_{K K}^{S}$ is fixed to

$N_{K K}$, satisfies $\int_{0}^{N_{\dot{K} K}^{9}} L_{\max }\left(N_{K K}\right) \mathrm{d} N_{K K} / \int_{0}^{\infty} L_{\max }\left(N_{K K}\right) \mathrm{d} N_{K K}=0.9$. For this upper limit we also reduce the efficiency by its systematic uncertainty. In the remainder of this section we perform a detail examination of these results, testing that the fit has behaved as expected by estimating the goodness of fit, comparing the errors of the fitted parameters with the toy $\mathrm{MC}$, and visually verifying the consistency of the PDFs and results with the data.

\subsubsection{Comparison with Toy Monte Carlos and Investigation of Possible Biases}

We assess the quality of the fit to the data by using the results in table 6.9 to generate and fit toy Monte Carlo experiments. This test allows us to quantify the likelihood of obtaining our data set, assuming our fit results are the true values of the fit parameters. In an alternative interpretation, we test whether our basic assumptions, which we have incorporated into the toy Monte Carlo, are compatible with our data set. Figure 6.7 displays the yield parameter errors and $-2 \log L=\chi^{2}-C$ (see section 5.2.1) for 500 such experiments. The arrows, which indicate the values obtained from the fit to the data, illustrate that the fit is typical. The $-2 \log L$ distribution suggests that the probability of obtaining a worst fit is $\approx 13 \%$.

We also examine distributions of the difference between the fitted yield parameters and their generated values in order to quantify any fit bias. As figure 6.8 illustrates, we find a bias of +1.2 events $(0.8 \%)$ on $N_{\pi \pi}^{S}$ and -3.3 events $(0.6 \%)$ on $N_{K \pi}^{S}$. This effect, which appears to shift events between different species, is not fully understood. The yields of the background species, which cannot be separated by $\Delta E$, suffer from 

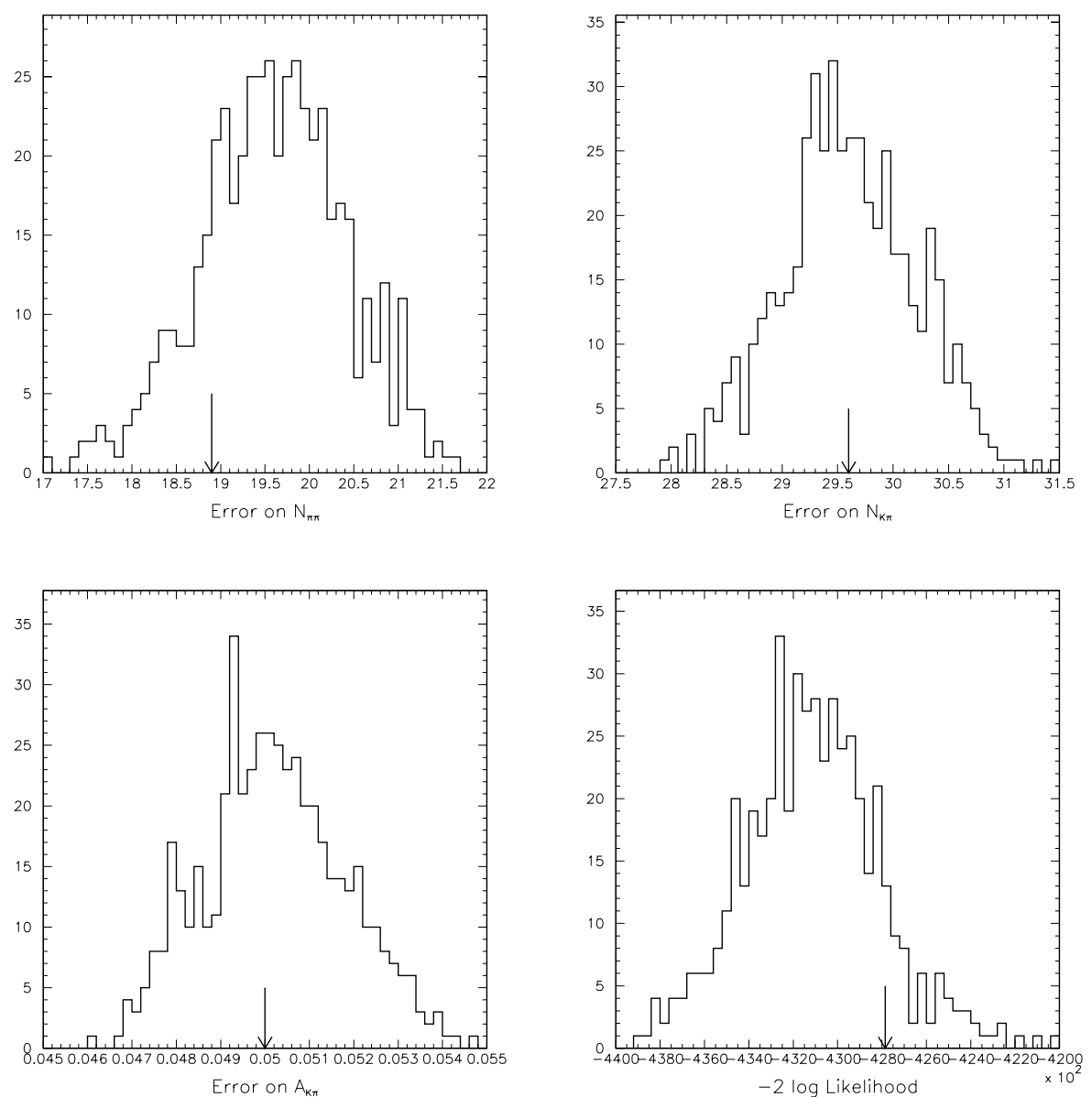

Figure 6.7: The distribution of fitted errors on $N_{\pi \pi}^{S}, N_{K \pi}^{S}$, and $\mathcal{A}_{K \pi}^{S}$, and the $-2 \log L=\chi^{2}-C$ of fits to 500 toy Monte Carlo experiments generated with the parameters obtained from the fit to the data. The errors indicate the result from the data fit. 

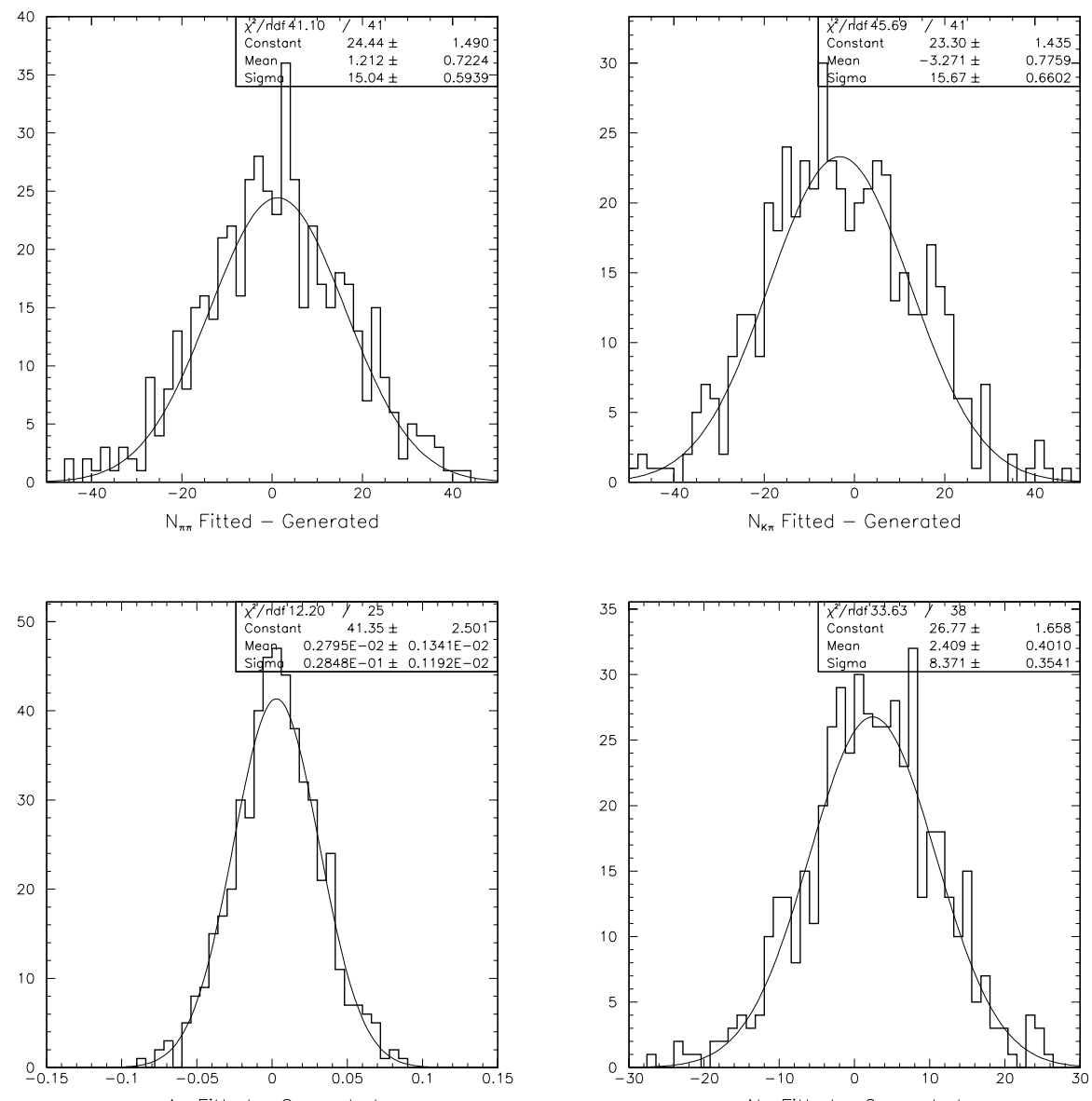

$A_{k \pi}$ Fitted - Generated

$N_{\text {KK }}$ Fitted - Generated

Figure 6.8: The distribution of the difference between fitted $N_{\pi \pi}^{S}, N_{K \pi}^{S}, \mathcal{A}_{K \pi}^{S}$, and $N_{K K}^{S}$ and inputed values for 500 toy Monte Carlo experiments generated with the parameters obtained from the fit to the data. 
a larger manifestation of this bias. This fact suggest that the source of the effect may originate in the DIRC PDFs. However studies have been unable to pin point whether it originates in generation, fitting, or a wrong assumption in the analysis. An independent fit/toy Monte Carlo, based on ROOT rather than LMinuit, has also observed the same bias. Since we cannot eliminate the toy Monte Carlo generation as the source of this effect, we choose not to correct for the bias and instead include the observed bias in the systematic error.

\subsubsection{Plots}

The production of plots which visually validate our measurement is complicated by our choice to extract results from a ML fit on a loosely selected sample in which the three different signal decays are mixed together along with a very large background. Certainly the $m_{\mathrm{ES}}$ and $\Delta E$ distributions in figure 5.7 are not very illuminating. Therefore, we choose to plot variable distributions after cuts which isolate the various signal decays in the manner described in the first paragraph of section 5.2. For comparison to the fit, we then overlay the PDFs after correcting for the selection efficiency.

We make the selections using likelihoods constructed from the PDFs used in the ML fit. When plotting variable $x$, we isolate signal by cutting on the likelihood

$$
L_{\mathrm{sig}}=\frac{P_{\pi \pi}^{S}+P_{K \pi}^{S}+P_{\pi K}^{S}+P_{K K}^{S}}{P_{\pi \pi}^{S}+P_{K \pi}^{S}+P_{\pi K}^{S}+P_{K K}^{S}+P_{\pi \pi}^{B}+P_{K \pi}^{B}+P_{\pi K}^{B}+P_{K K}^{B}},
$$

where $P_{i}^{S, B}$ is the product of all signal or background PDFs for species $i$, excluding the variable $x$. We further isolate an individual signal species $i$ by performing a second cut on the likelihood

$$
L_{i}=\frac{P_{i}^{S}}{P_{\pi \pi}^{S}+P_{K \pi}^{S}+P_{\pi K}^{S}+P_{K K}^{S}} .
$$

We determine the $L_{\text {sig }}$ and $L_{i}$ cut values which produce the most statistically signifi- 
cant selection, by maximizing

$$
S\left(L_{\mathrm{sig}}^{\prime}, L_{i}^{\prime}\right)=\frac{N_{i}\left(L_{\mathrm{sig}}^{\prime}, L_{i}^{\prime}\right)}{\sqrt{\sum_{j} N_{j}\left(L_{\mathrm{sig}}^{\prime}, L_{i}^{\prime}\right)}},
$$

where $N_{i}\left(L_{\text {sig }}^{\prime}, L_{i}^{\prime}\right)$ is the number of expected events of species $i$ in the $3 \sigma$ signal region of variable $x$ with $L_{\text {sig }}>L_{\text {sig }}^{\prime}$ and $L_{i}>L_{i}^{\prime}$. Since the Geant4 simulation does not adequately reproduce the data likelihoods, we rely on toy Monte Carlo events generated with the parameters of the data fit to estimate $N_{i}\left(L_{\text {sig }}^{\prime}, L_{i}^{\prime}\right)$.

Figure 6.9 plots the $m_{\mathrm{ES}}$ and $\Delta E$ distributions for samples enhanced in $B^{0} \rightarrow$ $\pi^{+} \pi^{-}$or $B^{0} \rightarrow K^{+} \pi^{-}$decays, overlayed with the appropriate PDFs. Similarly figure 6.10 displays the Fisher distribution for a sample enhanced in signal decays.

\subsubsection{Consistency of Fitted Signal Parameters}

Though we choose to fix the parameters of the signal PDFs in our nominal fit, we check the consistency of our estimates of their values by directly measuring these parameters in separate fits to the data sample. Table 6.10 summarizes the results of fits where the signal $m_{\mathrm{ES}}, \Delta E$, and $\mathcal{F}$ parameters were floated. In all cases we find that the direct fits are consistent with their values in the nominal fit.

\subsection{Systematics}

Table 6.11 lists the estimated systematic errors on the measured yields due to PDF parameterizations. For the signal $m_{\mathrm{ES}}, \Delta E$, and $\mathcal{F}$ PDFs, we calculated the systematics by noting the shift in the fitted yield and asymmetries after varying each parameter within its estimated uncertainties. $\mathcal{F}^{3 \mathrm{G}}$ refers to the possible bias resulting from events on the signal side beyond the double Gaussian tail in the Fisher discriminant, discussed in section 6.1.1. As described in section 6.1.1, we varied $\theta_{c}$ parameters 

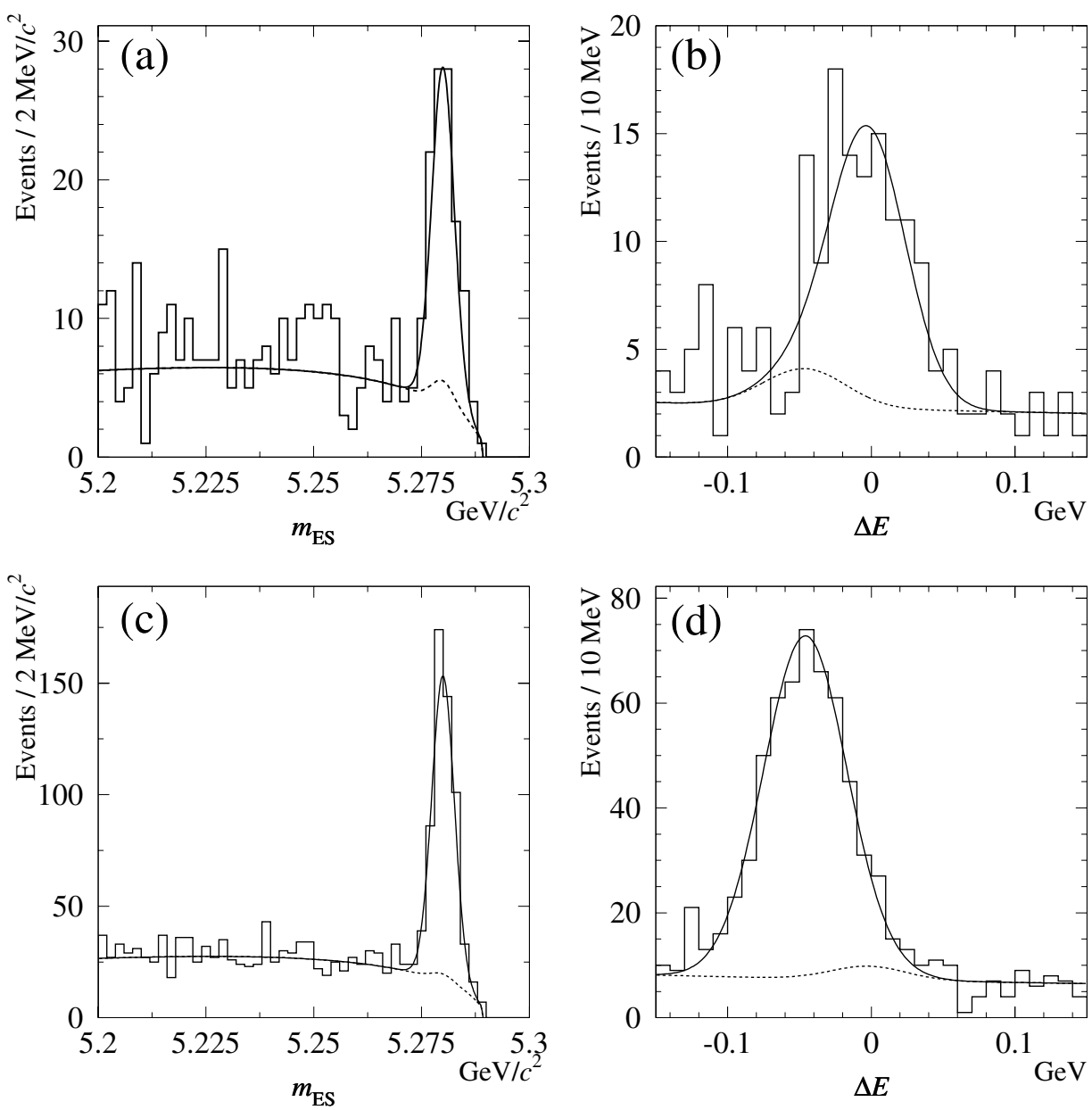

Figure 6.9: Distributions of $m_{\mathrm{ES}}$ and $\Delta E$ for samples enhanced in (a,b) signal $\pi^{+} \pi^{-}$ and (c,d) $K^{\mp} \pi^{ \pm}$decays. Solid curves represent projections of the maximum likelihood fit, dashed curves represent $q \bar{q}$ and $\pi \pi \leftrightarrow K \pi$ cross-feed background. 
Table 6.10: Comparison of the nominal values of signal PDF parameters and their fitted values on the data. The errors listed on the nominal values are used in the evaluation of the systematic error.

\begin{tabular}{lccc}
\hline \hline Fit & Parameter & Nominal Value & Fitted Value \\
\hline Signal & $\mu_{m_{\mathrm{ES}}}$ & $5.2800 \pm 0.0005 \mathrm{GeV} / c^{2}$ & $5.2802 \pm 0.0001 \mathrm{GeV} / c^{2}$ \\
$m_{\mathrm{ES}}$ & $\sigma_{m_{\mathrm{ES}}}$ & $2.5 \pm 0.1 \mathrm{MeV} / c^{2}$ & $2.58 \pm 0.11 \mathrm{MeV} / c^{2}$ \\
& & & \\
Signal & $\mu_{\Delta E}$ & $-2_{-2}^{+1} \mathrm{MeV}$ & $-1.3 \pm 1.5 \mathrm{MeV}$ \\
$\Delta E$ & $\sigma_{\Delta E}$ & $26_{-0.7}^{+1.0} \mathrm{MeV}$ & $28.1 \pm 1.4 \mathrm{MeV}$ \\
& & & \\
Signal & $\mu_{\mathcal{F}}$ & $0.00929 \pm 0.01094$ & $0.08549 \pm 0.07006$ \\
$\mathcal{F}$ & $\sigma_{\mathcal{F}}^{L}$ & $0.69909 \pm 0.00676$ & $0.75952 \pm 0.04251$ \\
& $\sigma_{\mathcal{F}}^{R}$ & $0.39737 \pm 0.00699$ & $0.37228 \pm 0.05109$ \\
\hline \hline
\end{tabular}




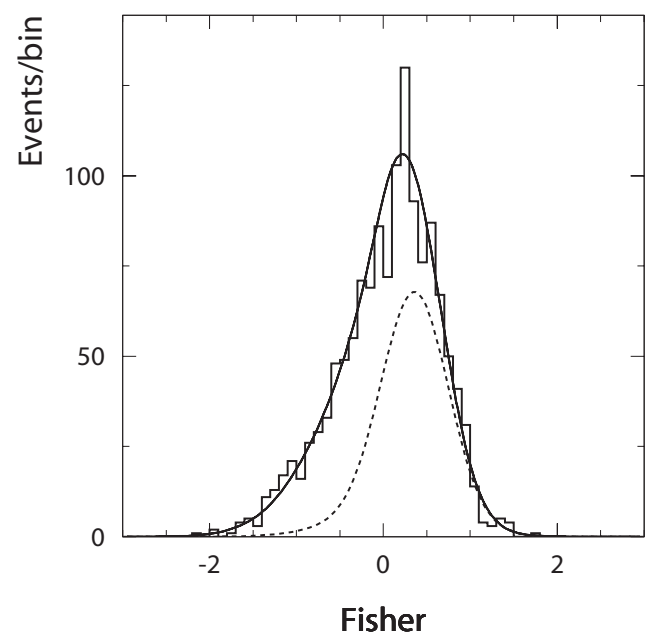

Figure 6.10: The Fisher discriminant distribution for a sample enhanced in signal decays. The solid curve represents the projection of the maximum likelihood fit. The dashed curve represents the signal contribution.

coherently. Table 6.12 summarizes all of the systematics considered along with their quadrature sum. In addition to the uncertainties listed table 6.11, we include

- Fit Bias - We account for the possibility of a fit bias of $1.6 \%$ for $\pi \pi$ and $0.7 \%$ for $K \pi$ based on the fits to pure signal Monte Carlo samples in table 6.6. We consider this to be conservative, since the signal migration appears to be related to PID and we have already accounted for variations in the $\theta_{c}$ PDFs.

- Toy MC bias - We apply any bias observed in the nominal toy Monte Carlo configuration as a systematic error (section 6.2.1).

- Detector Charge Bias - Since the $h^{+} h^{-}$final state includes one track of each charge, our measurements are unaffected by any charge asymmetry in the tracking efficiency. However any potential charge dependence in the particle identification could bias $\mathcal{A}_{K \pi}$. We estimate an upper bound on such an effect by 
comparing different measurements of the asymmetry $\mathcal{A}_{D}=\left(N_{D^{0}}-N_{\bar{D}^{0}}\right) /\left(N_{D^{0}}+\right.$ $N_{\bar{D}^{0}}$ ) in the $D^{*}$ control sample (see section 5.1.3). We measure $\mathcal{A}_{D}[69]$

- kinematically using the correlation of the kaon charge with the slow pion, but no other selections,

- kinematically when both tracks satisfy the $\theta_{c}$ selections in section 5.3.2, and

- using the DIRC in a ML fit to the $D^{0}$ mass and the $\theta_{c}$ measurements.

A conservative estimate of the variation between these measurements leads to a systematic error of \pm 0.01 on $\mathcal{A}_{K \pi}$.

- Efficiency and $\mathrm{N}_{\mathrm{B} \overline{\mathrm{B}}}$ - These are the estimated error on the determination of the efficiency (see table 5.1 and section 4.2.1) and the number of $B \bar{B}$ events (see section 4.3.2).

\subsection{Summary}

Table 6.13 lists our measurements of the branching fractions, including the systematic errors. The $B^{0} \rightarrow K^{+} K^{-}$upper limit is increased by the systematic error on its yield. We measure the direct CP asymmetry in $B^{0} \rightarrow K^{+} \pi^{-}$to be $-0.102 \pm 0.050 \pm 0.016$. We find $-0.188<\mathcal{A}_{K \pi}^{S}<-0.016$ at $90 \%$ confidence level. 
Table 6.11: Detailed summary of systematic errors on yields and CP asymmetries due to uncertainty in the parameterization of the PDFs.

\begin{tabular}{cccccccccc}
\hline \hline Source & \multicolumn{2}{c}{$N_{\pi \pi}(\%)$} & \multicolumn{2}{c}{$N_{K \pi}(\%)$} & \multicolumn{2}{c}{$\mathcal{A}_{K \pi}$} & \multicolumn{2}{c}{$N_{K K}$} \\
\hline$\mu_{\Delta E}$ & -1.58 & +0.68 & -0.29 & +0.40 & -0.0015 & +0.0007 & -1.03 & +0.46 \\
$\sigma_{\Delta E}$ & -1.21 & +0.62 & -1.30 & +0.63 & -0.0007 & +0.0000 & -0.93 & +0.45 \\
$\mu m_{\mathrm{ES}}$ & -1.31 & +0.81 & -0.31 & +0.00 & -0.0022 & +0.0022 & -0.80 & +0.96 \\
$\sigma_{m_{\mathrm{ES}}}$ & -1.38 & +1.17 & -0.96 & +0.80 & -0.0006 & +0.0005 & -0.11 & +0.02 \\
$\mu_{\mathcal{F}}$ & -0.91 & +0.91 & -0.56 & +0.54 & -0.0007 & +0.0007 & -0.05 & +0.02 \\
$\mathcal{F}^{3 \mathrm{G}}$ & 0.00 & +2.55 & 0.00 & +1.35 & 0.0000 & +0.0050 & -7.00 & 0.00 \\
$\sigma_{\mathrm{L}}^{\mathcal{F}}$ & -0.35 & +0.34 & -0.20 & +0.20 & -0.0002 & +0.0002 & -0.85 & +0.84 \\
$\sigma_{\mathrm{R}}^{\mathcal{F}}$ & -0.50 & +0.51 & -0.32 & +0.31 & -0.0005 & +0.0005 & -0.12 & +0.11 \\
$\mathcal{F}^{3 \mathrm{G}}$ & 0.00 & +2.55 & 0.00 & +1.35 & 0.0000 & +0.0050 & -7.00 & 0.00 \\
$\theta_{c}^{K^{-}}$ & -1.45 & +0.10 & -0.03 & +0.47 & -0.0045 & +0.0036 & -0.02 & +0.28 \\
$\theta_{c}^{K^{+}}$ & 0.00 & +1.42 & -0.44 & +0.00 & -0.0034 & +0.0046 & -0.12 & +0.19 \\
$\theta_{c}^{\pi^{-}}$ & -0.17 & +1.77 & -0.05 & +0.14 & -0.0065 & +0.0066 & -0.80 & +0.26 \\
$\theta_{c}^{\pi^{+}}$ & -1.83 & +0.00 & -0.13 & +0.06 & -0.0063 & +0.0072 & 0.00 & +1.11 \\
\hline \hline
\end{tabular}

Table 6.12: Global summary of systematic errors on branching fractions and CP asymmetry.

\begin{tabular}{ccccccccc}
\hline \hline Source & \multicolumn{2}{c}{$\mathcal{B}_{\pi \pi}(\%)$} & \multicolumn{2}{c}{$\mathcal{B}_{K \pi}(\%)$} & \multicolumn{2}{c}{$\mathcal{A}_{K \pi}$} & \multicolumn{2}{c}{$N_{K K}$} \\
\hline$m_{\mathrm{ES}}$ & -1.90 & +1.42 & -1.01 & +0.80 & -0.0023 & +0.0022 & -0.81 & +0.96 \\
$\Delta E$ & -1.99 & +0.92 & -1.33 & +0.75 & -0.0017 & +0.0007 & -1.39 & +0.64 \\
$\mathcal{F}$ & -1.10 & +2.78 & -0.68 & +1.50 & -0.0009 & +0.0051 & -7.1 & +0.85 \\
$\theta_{c}$ & -2.34 & +2.28 & -0.46 & +0.49 & -0.0107 & +0.0114 & -0.81 & +1.19 \\
Fit bias & 0.00 & +1.6 & -2.00 & 0.00 & -0.0005 & 0.0000 & 0.00 & 0.00 \\
Toy MC bias & 0.00 & +0.77 & -0.56 & 0.00 & 0.0028 & 0.0028 & 0.00 & +2.40 \\
Det $q$ bias & 0.00 & 0.00 & 0.00 & 0.00 & -0.01 & +0.01 & 0.00 & 0.00 \\
efficiency & -2.0 & +2.0 & -2.0 & +2.0 & 0.0000 & 0.0000 & $-2.0 \%$ & $+2.0 \%$ \\
$N_{B \bar{B}}$ & -1.1 & +1.1 & -1.1 & +1.1 & 0.0000 & 0.0000 & $-1.1 \%$ & $+1.1 \%$ \\
\hline Total & -4.4 & +4.9 & -3.6 & +3.0 & -0.0152 & +0.0164 & -8.9 & +3.0 \\
\hline \hline
\end{tabular}


Table 6.13: Summary of branching fraction results in the on-resonance $(87.9 \pm 1.0$ million $B \bar{B}$ pairs). The errors are statistical and systematic, respectively.

\begin{tabular}{lccc}
\hline \hline Mode & $N_{S}$ & $\epsilon(\%)$ & $\mathcal{B}\left(10^{-6}\right)$ \\
\hline$\pi^{+} \pi^{-}$ & $156.5 \pm 18.9 \pm 6.5$ & $37.98 \pm 0.76$ & $4.6 \pm 0.6 \pm 0.2$ \\
$K^{+} \pi^{-}$ & $588.5 \pm 29.6_{-16.5}^{+11.3}$ & $37.48 \pm 0.76$ & $17.9 \pm 0.9 \pm 0.7$ \\
$K^{+} K^{-}$ & $0.8 \pm 7.7(<15.9)$ & $36.23 \pm 0.73$ & $<0.6(90 \%$ C.L. $)$ \\
\hline \hline
\end{tabular}




\section{Chapter 7}

\section{Ingredients of a Time-dependent CP}

\section{Analysis}

In chapter 2 we found that the time evolution of the $B^{0} \bar{B}^{0}$ system, when one of the $B$ 's decays into a CP eigenstate is described by

$$
\Gamma_{\bar{B}^{0}}^{B^{0}}(\Delta t)=\frac{e^{-\frac{|\Delta t|}{\tau}}}{4 \tau}\left(1 \pm S_{f} \sin \left(\Delta m_{d} \Delta t\right) \mp C_{f} \cos \left(\Delta m_{d} \Delta t\right)\right),
$$

where $\Gamma_{\bar{B}^{0}}^{B^{0}}$ corresponds to the flavor of the other $B^{0}\left(\bar{B}^{0}\right)$, and $S_{f}$ and $C_{f}$ measure CP violation. Having isolated candidate $B^{0} \rightarrow h^{+} h^{\prime-}$ decays, we now turn our attention to extracting these parameters from the $B^{0} \rightarrow \pi^{+} \pi^{-}$decays. Equation (7.1) requires two inputs: the flavor of the other $B$ meson in our signal events and the difference, $\Delta t$, in the decay times of the two $B$ mesons. In this chapter we'll discuss the strategy for measuring these quantities. In the next chapter, we will add these new analysis ingredients to the branching fraction analysis to extract $S_{\pi^{+} \pi^{-}}$and $C_{\pi^{+} \pi^{-}}$.

Prior to the time-dependent analysis of the $B^{0} \rightarrow \pi^{+} \pi^{-}$decay, $b$-flavor tagging and $\Delta t$ extraction were used in the measurement of $\Delta m_{d}$ and $\sin 2 \beta$. For this analysis we adopted the same techniques, which, having been developed for a more precise measurements, are more than adequate. Both methods avoid the inefficiencies of 
the exclusive reconstruction of the other meson by inclusively inferring its flavor and decay vertex from its final decay products. In order to assess their performance, the flavor mis-tag probabilities and vertexing resolution are measured on a sample of fully reconstructed $B$ decays to self-tagging final states, where the physics of the flavor and time structure of the events are known.

\section{$7.1 \quad b$-Flavor Tagging}

Neutral $B$ mesons often decay to final states, which are only accessible to either a $b$ or $\bar{b}$-quark, therefore revealing the meson's flavor. For example, a positively charged lepton from $B^{0} \rightarrow D^{(*)-} l^{+} \nu$ identifies the presence of a $\bar{b}$-quark and allows the meson to be tagged as a $B^{0}$. Despite the impressively large number of $B$ decays recorded by $B A B A R$, the reconstruction efficiency of such self-tagging modes, coupled with the small branching fraction of CP final states, produces insufficient yields of exclusively reconstructed $B \bar{B}$ pairs for any time-dependent measurement. However inclusive methods, which using kinematic and particle identification select particles who's charges are likely to correlate with the $b$-quark flavor, provide adequate $b$-flavor determination for CP measurements.

$B A B A R$ 's flavor tagger is designed to exploit correlations between the $b$-flavor and the charges of the final products of four distinct $b$-quark decay modes. It is tuned and tested on Monte Carlo events where the flavor of the $B$ mesons are known. However, since the Monte Carlo does not perfectly mimic the data recorded by the detector, the performance of the tagger is assessed in samples of fully reconstructed $B$ decays. The influence of the tagger on measured time-dependent asymmetries is quantified in the parameter $Q=\epsilon^{t}(1-2 w)^{2}=\epsilon^{t} D^{2}$, which takes into account the tagging efficiency 
$\left(\epsilon_{T}\right)$ and the probability of mis-identifying the flavor of the $B$ meson $(w)$.

\subsubsection{Flavor Tagging Decays}

The $b$-flavor tagging algorithm employs two layers of decisions, labeled sub-net and dispatch-net due to their heavy use of neural networks, to recognize different sources of flavor carrying tracks and then combine all available information to determine a best tag of a $B$ meson's flavor. Seven independent sub-nets use kinematic and particle identification information to identify the signature of four specific flavor revealing processes. We will survey these processes in this section.

\section{Leptons from Semi-leptonic Decays}
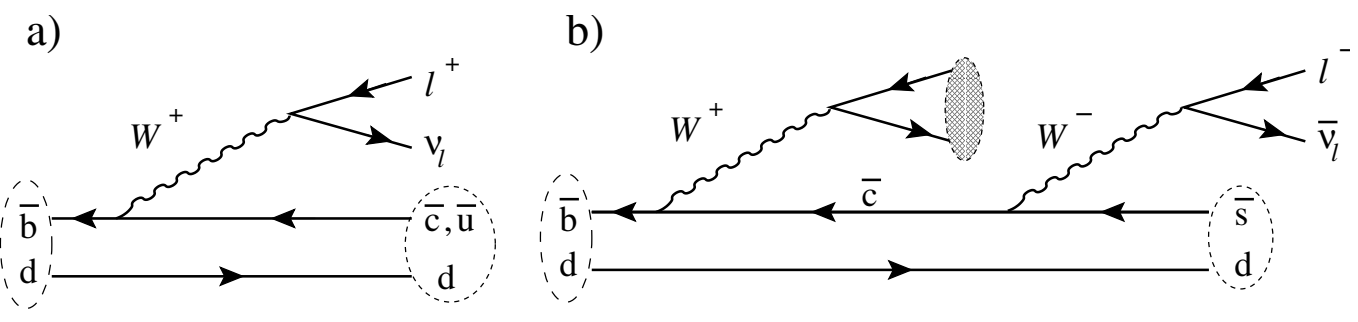

Figure 7.1: Leading lepton producing neutral $B$ meson decays. The $b$-quark and lepton charges are correlated in (a) $B \rightarrow X l \nu$ (a) and anti-correlated in the (b) $\bar{b} \rightarrow \bar{c} \rightarrow \bar{s}$.

Semi-leptonic $B \rightarrow X l \nu$ decays (figure $7.1 \mathrm{a}$ ), which constitute roughly $20 \%$ of the $B$ branching fraction, produce electrons or muons whose charge has same sign as the $b$-quark. Since these leptons are the primary product of the virtual $W$ boson emitted by the $b$-quark, they carry large momenta $p_{l}^{*}$ in the center of mass of the $B$ and may therefore be distinguished from softer secondary leptons from $\bar{b} \rightarrow \bar{c} \rightarrow \bar{s}$ transitions (figure 7.1b) which exhibit the opposite lepton/b-quark correlation. The 
primary leptons are also faster than most pions and kaons produced by $B$ decays, allowing additional discrimination of misidentified leptons and also permitting purely kinematic selection of the $B \rightarrow X l \nu$ lepton when no particle identification is available.

Three separate neural network sub-nets recognize primary leptons, two of which examine identified electrons or muons, and one which only considers kinematics. In addition to $p_{l}^{*}$, these three sub-nets also moderately benefit from two other kinematic variables: the total energy in the hemisphere defined by $W$ direction, which is generally smaller for $B \rightarrow X l \nu$ than its inclusive backgrounds, and the CM angle between missing momentum (i.e. the $\nu$ direction) and the primary lepton, which is also small for real semi-leptonic decays. Ultimately kinematics and strict lepton identification render the semi-leptonic $B$ decays the cleanest and hence most reliable flavor tagging signature. As we shall see in table 7.8, though tagging using leptons is not very efficient $\left(\epsilon_{1}^{t} \approx 9 \%\right)$, it is very accurate $\left(w_{1} \approx 3 \%\right)$, resulting in $Q_{1} \approx 0.08$.

\section{Kaons from $b \rightarrow c \rightarrow s$}

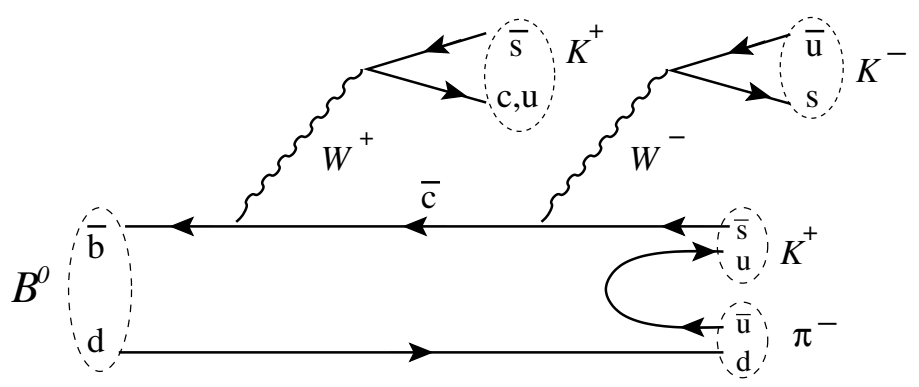

Figure 7.2: An example of a $\bar{b} \rightarrow \bar{c} \rightarrow \bar{s}$ transition which produces kaons whose charge has both the same and opposite sign as the $b$-quark.

The correlation of final state kaons and the $b$-quark charge is illustrated by the observation that the average number of positively charged kaons (the $K^{+}$multiplic- 
ity) in $B^{0}$ decay products is $0.58 \pm 0.01 \pm 0.08$, while the negatively charged kaon multiplicity is $0.13 \pm 0.01 \pm 0.05$ [70]. Figure 7.2 displays an example a of $\bar{b} \rightarrow \bar{c} \rightarrow \bar{s}$ transition which produces each of the three kaon sources which dominate to this observation. The $\bar{s}$-quark in the $\bar{b} \rightarrow \bar{c} \rightarrow \bar{s}$ cascade is the primary source of the positive (or right-sign) correlation between the b-quark and kaon charge. However, this cascade also emits two virtual $W$-bosons which occasionally also hadronize to kaons. In the specific process diagramed in figure 7.2 , the $W^{+}$boson produces a Cabbibo suppressed $u \bar{s}$, which results in another right-sign kaon. Similarly, the $W^{-}$-boson from the $\bar{c}$ decay results in a wrong-sign kaon. Unlike the primary leptons, no kinematic separation between the right and wrong sign kaons has been observed, leaving kaon identification as the only signature. Therefore the tagger identifies kaons using one sub-net which examines the three best kaon candidates and determines the $b$ flavor from the sum of the product of each kaon's charge and likelihood to be a kaon, which is calculated using the DCH $d E / d x$ and DIRC $\theta_{c}$ measurements. Flavor tags from kaons are generally more efficient than lepton tags, but less accurate. Looking ahead at table 7.8 , we see that the best kaon tagged events have $\epsilon_{2}^{t} \approx 17 \%$ and $D_{2} \approx 0.8$ resulting in $Q_{2} \approx 0.11$.

\section{Soft Pions from $D^{* \pm}$ Decays}

In the decay $D^{*+} \rightarrow D^{0} \pi^{+}$, the $D^{*+}$ and $D^{0}$ masses are so close $\left(\approx 142 \mathrm{MeV} / c^{2}\right)$ that the resulting pion carries very little momentum and flies in the same direction as the $D^{0}$. This pion is usually described as slow or soft. When the $D^{*}$ originates from a $B$ meson decay, as in figure 7.3 , the $D^{*}$ 's charge and hence its slow-pion's charge are opposite to that of the original $b$-quark. The slow-pion sub-net is a neural network which examines pions with CM momentum $p_{\pi_{s}}^{*}$ less than $250 \mathrm{MeV}$ and identifies a slow- 


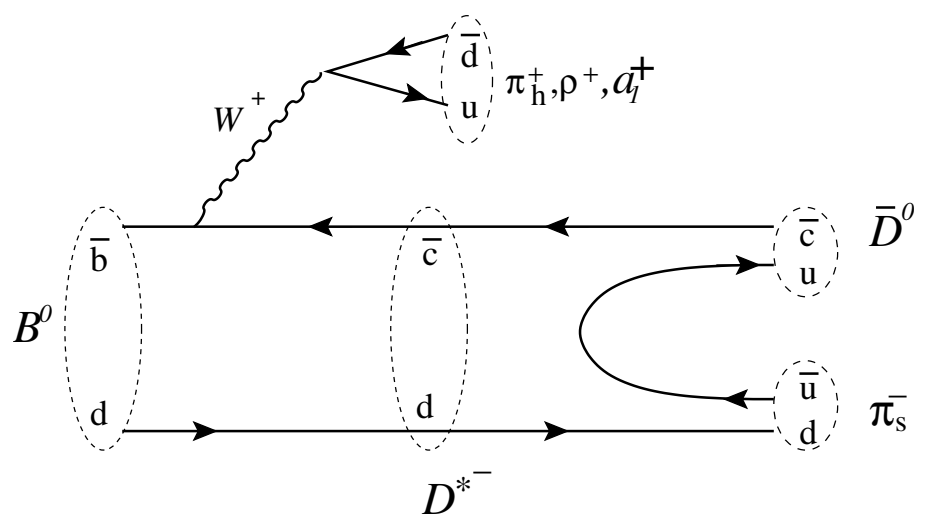

Figure 7.3: $B^{0} \rightarrow D^{*-} \pi^{+}, \rho^{+}, a_{1}^{+}$decays which produce a soft pion whose charge has the opposite sign as the $b$-quark. In contrast the $\pi^{+}, \rho^{+}$, or $a_{1}^{+}$from the $B$ carries the same charge as the $b$-quark.

pion from its momentum $p_{\pi_{s}}^{*}$, the angle between its flight direction and the thrust axis of the rest of the $B$ meson products, and particle identification information.

Another sub-set attempts to exploit correlations between the kaon and slow-pion from the $D^{*}$ to produce a more reliable tag. This neural network-based sub-net examines all oppositely charged slow pion and kaon combinations along with the kaon likelihood, the slow-pion sub-net output, and the angle between the kaon and slow-pion.

\section{Hard Pions from $B^{0} \rightarrow D^{*-} \pi^{+}, \rho^{+}, a_{1}^{+}$Decays}

The charge of the virtual $W^{+}$boson in figure 7.3 carries the same sign as the $b$ quark charge. When this boson hadronizes into a pion, the $b$-quark flavor may be identified from the characteristically fast momentum of prompt $B$ meson products. A maximum $p^{*}$ sub-net attempts to capture the $b$-flavor from these particles by selecting the track with the highest $\mathrm{CM}$ momentum which originate from less than $1 \mathrm{~mm}$ in the 
$x-y$ plane from the beam. This procedure also captures prompt leptons which were missed by the lepton sub-nets and which fortunately have the same charge/b-flavor correlation.

\subsubsection{Combining Tag Signatures}

A given set of particles belonging to a $B$ meson may exhibit the signature of any number of the four flavor-tagging physics processes in section 7.1.1, and therefore may be identified by several of the seven sub-nets. In general, each sub-net $i$ may provide an output $r_{1}^{i}$ whose sign and magnitude reflect the $B$ flavor and the confidence in the result. The dispatch-net attempts to optimally combine these outputs in order to produce an output $r_{2}$ which captures the most reliable tag of the meson flavor considering all available information.

The sub-net and dispatch-net outputs, $r_{1}^{i}$ and $r_{2}$, are fed to a decision algorithm which assigns the tag to one of nine hierarchical physics-signature categories (in descending order of reliability): electron-kaon, muon-kaon, electron, muon, kaon-slow pion, kaon I, slow pion, kaon II, and other (mostly hard pions). The presence of two kaon categories reflects the wide range of reliability of the kaon sub-net's output. Events which do not satisfy the requirements of any of these categories are marked as untagged.

Unfortunately assessing the performance of nine different types of $B$ flavor tags is impractical. The number of events populating some categories is too little and too many parameters are required for reliable fits to current data samples. Therefore these physics-signature categories are regrouped into five tagging categories based on the similar flavor tagging ability as follows: category 1- electron-kaon, muon-kaon, electron, muon; category 2- kaon-slow pion, kaon I; category 3- slow pion, kaon II, 
category 4- other; and category 5- untagged.

\subsubsection{Tagging Imperfections}

The Monte Carlo event generator's incomplete knowledge of $B$ branching fractions and the Geant4 simulation's imperfect reproduction of the detector's response warrants the extraction of parameters which quantify the tagger's performance from data recorded by the detector. This is made possible through the recognition that $B^{0}$ mixing may be exploited to measure tagging parameters. The arguments in section 2.23, when applied to $B$ decays to a flavor eigenstate, lead to the time-dependent probability distributions for four different possible flavor combinations:

$$
\begin{aligned}
& \Gamma_{B^{0}, \bar{B}^{0}}(\Delta t)=\Gamma_{\bar{B}^{0}, B^{0}}(\Delta t)=\frac{e^{-\frac{|\Delta t|}{\tau}}}{4 \tau}\left(1+\cos \left(\Delta m_{d} \Delta t\right)\right), \\
& \Gamma_{B^{0}, B^{0}}(\Delta t)=\Gamma_{\bar{B}^{0}, \bar{B}^{0}}(\Delta t)=\frac{e^{-\frac{|\Delta t|}{\tau}}}{4 \tau}\left(1-\cos \left(\Delta m_{d} \Delta t\right)\right),
\end{aligned}
$$

where the deviations from the exponent, the $\cos \left(\Delta m_{d} \Delta t\right)$ terms, are due to mixing. These two decay distributions are usually referred to as the unmixed and mixed probabilities, respectively.

For the extraction of the tagging performance, one $B$ meson is fully reconstructed, so its flavor is known. The tagger then is supplied the particles which are not part of this meson in order to determine the flavor of the other $B$. Several imperfections in this procedure modify (7.2). In particular for a tagging category $i$ :

1. The tagging algorithm may produce a wrong-tag. We'll denote this mis-tag probability as $\bar{w}_{i}$.

2. There may be different mis-tag probabilities, $w_{i+}$ and $w_{i-}$, for $B^{0}$ and $\bar{B}^{0}$ tags. So $\bar{w}_{i}=\left(w_{i+}+w_{i-}\right) / 2$. 
3. There may be different efficiencies, $\epsilon_{i+}^{t}$ and $\epsilon_{i-}^{t}$, for tagging a $B^{0}$ and $\bar{B}^{0}$.

4. There may be different efficiencies, $\epsilon_{i+}^{r}$ and $\epsilon_{i-}^{r}$, for fully reconstructing a $B^{0}$ and $\bar{B}^{0}$.

5. There is an inherent resolution in the measurement of $\Delta t$. We will address this issue in section 7.3 .

Using these definitions and (7.2), the probability distribution for observing an event with a tagged flavor $T= \pm\left(+=B^{0},-=\bar{B}^{0}\right)$ for one meson and reconstructed flavor $R= \pm$ for the other is

$$
P_{i}(\Delta t, T, R)=\frac{\epsilon_{i(R)}^{r}}{\epsilon_{i(R)}^{r}+\epsilon_{i(-R)}^{r}}\left[\epsilon_{i(T)}^{t}\left(1-w_{i(T)}\right) \Gamma_{(R),(T)}+\epsilon_{i(-T)}^{t} w_{i(-T)} \Gamma_{(-R),(T)}\right]
$$

where the first term in the sum is for the correct $(T, R)$ tags and the second term accounts for mis-tags which are actually $(-T, R)$. Reorganizing this expression and properly normalizing in each category $^{1}$, leads to

$$
\begin{aligned}
P\left(\Delta t, T, R ; \hat{q}_{i}\right)= & \frac{\bar{\epsilon}_{i}^{t}}{8 \tau} \frac{1+R \nu_{i}}{1-\mu_{i} \nu_{i} \xi} e^{-\frac{|\Delta t|}{\tau}} \times \\
& \left(\left[\mu_{i} T D_{i}+B_{i}\right]-\left[T D_{i}+\mu_{i} B_{i}\right] R \cos \left(\Delta m_{d} \Delta t\right)\right), \\
& B_{i}=\left(1+T \frac{\Delta D_{i}}{2}\right),
\end{aligned}
$$

where $\xi \equiv \frac{1}{1+\left(\tau \cdot \Delta m_{d}\right)^{2}}$ and the parameters $\hat{q}_{i}$ are

- $D_{i} \equiv 1-2 \bar{w}_{i}$ which is known as the dilution,

- the dilution difference $\Delta D_{i} \equiv 2\left(w_{i-}-w_{i+}\right)$,

\footnotetext{
${ }^{1}$ The normalization requires that the probabilities of observing each of the four flavor combina-
} tions in each category add to the probability of tagging in that category, i.e. $\sum_{T, R} P_{i}(\Delta t, T, R)=\epsilon_{i}^{t}$. See 8.2.2 for more details. 
- the reconstruction efficiency asymmetries $\nu_{i} \equiv \frac{\epsilon_{i+}^{r}-\epsilon_{i}^{r}}{\epsilon_{i+}^{r}+\epsilon_{i-}^{r}}$,

- tagging efficiency asymmetries $\mu_{i} \equiv \frac{\epsilon_{i-}^{t}-\epsilon_{i-}^{t}}{\epsilon_{i+}^{t}+\epsilon_{i-}^{t}}$, and

- the average tagging efficiency $\bar{\epsilon}_{i}^{t} \equiv \frac{1}{2}\left(\epsilon_{i+}^{t}+\epsilon_{i-}^{t}\right)$.

The effect of tagging on time-dependent CP measurements, where the flavor of the fully reconstructed $B$ meson is unknown, is similar. Incorporating the tagging flaws into (7.1) leads to the probability distributions

$$
\begin{aligned}
P\left(\Delta t, T ; \hat{q}_{i}\right)= & \frac{\bar{\epsilon}_{i}^{t}}{4 \tau} \frac{1}{1-\mu_{i} \xi C_{f}} e^{-\frac{|\Delta t|}{\tau}} \times \\
& \left(\left[\mu_{i} T D_{i}+\left(1+T \frac{\Delta D_{i}}{2}\right)\right]+\left[T D_{i}+\mu_{i}\left(1+T \frac{\Delta D_{i}}{2}\right)\right] A(\Delta t)\right)
\end{aligned}
$$

where $A(t) \equiv S_{f} \sin \left(\Delta m_{d} \Delta t\right) \mp C_{f} \cos \left(\Delta m_{d} \Delta t\right)$. Setting $\nu_{i}=\mu_{i}=\Delta D_{i}=0$ in this equation illustrates the result of mistakes by the tagging algorithm. In this case,

$$
P_{i}(\Delta t, T)=\frac{\bar{\epsilon}_{i}^{t}}{4 \tau} e^{-\frac{|\Delta t|}{\tau}}\left[1+T D_{i}\left(S_{f} \sin \left(\Delta m_{d} \Delta t\right) \mp C_{f} \cos \left(\Delta m_{d} \Delta t\right)\right)\right] .
$$

and the only change in the functional form of (7.1) is the suppression of the amplitude of the sine and cosine by $D_{i}$. This effect, which is the dominate by-product of the tagging algorithm, dilutes the difference between $B^{0}$ and $\bar{B}^{0}$ tags. To a good approximation, the error on the determination of $S_{f}$ and $C_{f}$ in category $i$ is inversely proportional to $Q_{i} \equiv \epsilon_{i}^{t} D_{i}^{2}$.

\subsection{Measurement of $\Delta t$}

The two-body $\Upsilon(4 s) \rightarrow B \bar{B}$ decay produces back-to-back $B$ mesons with an average momentum of $340 \mathrm{MeV}$ in the $\mathrm{CM}$. With a lifetime of $\approx 1.5 \mathrm{ps}$ and $\beta \gamma \approx 0.06$, these $B \mathrm{~s}$ separate by only $\approx 34 \mu \mathrm{m}$ along each Cartesian axis before decaying. However in the 
lab frame, which is boosted in the $z$ direction by $\beta \gamma=.55$, the mean difference $\Delta z$ in

the $z$ coordinates of these decay vertices extends to $\approx 260 \mu \mathrm{m}$, a distance which is by design larger than the SVT's vertex resolution and therefore permits a measurement of $\Delta z$. The conversion of this $\Delta z$ to the time difference between the decays of the two $B, \Delta t$, is then a simple special relativity problem.

The full reconstruction of the $B$ meson provides an accurate measurement of its decay vertex, producing a resolution of $\approx 30-50 \mu \mathrm{m}$, depending on the final state. However, since exclusively reconstructing the other $B$ meson is not practical, its decay vertex is inclusively determined from a subset of its final state tracks and kinematic constraints from the $\Upsilon(4 s)$ decay. This procedure leads to a typical vertex resolution of $\approx 180 \mu \mathrm{m} . \Delta z$ is then calculated from the distance between the vertices of the fully reconstructed $B$ meson, $B_{\text {Rec }}$, and its inclusively reconstructed partner, $B_{\text {Tag. }}$. Clearly its resolution is dominated by $B_{\text {Tag }}$.

\subsubsection{The Algorithm}

$\Delta t$ is calculated in three which successively add information: the determination of the $B_{\operatorname{Rec}}$ vertex, the fit for the $\Delta z$, and the conversion to $\Delta t$. This section briefly outlines this procedure.

\section{Determination of the $B_{\operatorname{Rec}}$ Vertex}

Reconstructing a $B$ candidate begins with the search for possible intermediate decay products such as $D$ mesons or neutral pions and kaons in track and neutral cluster combinations in an event. Eventually the particles contributing to the decay are identified in the form of a tree representing all decays. The vertices are then simultaneously found in a fit which alters the momentum vector of the tracks and neutrals 
with appropriate constraints on the masses and directions of the composite particles. Neutral particles reconstructed in the EMC do not contribute to the vertex determination due to the lack of spatial information near their production. The procedure for finding the best vertex for $B^{0} \rightarrow h^{+} h^{\prime-}$ candidates described in section 5.3.2 is a simple application of this technique.

\section{Fit for the $B_{\text {Tag }}$ Vertex}

The $B_{\text {Tag }}$ vertexing algorithm examines the tracks which were not used in the reconstruction of $B_{\text {Rec }}$. Though these particles are generally the final products of the $B_{\text {Tag }}$ decay, those from intermediate states with long lifetimes do not originate from the $B$ decay vertex and must be eliminated. Therefore, oppositely charged track pair combinations are removed when they are consistent with $K_{s} \rightarrow \pi^{+} \pi^{-}$or $\lambda^{+} \rightarrow p^{+} \pi^{-}$ decays or $\gamma \rightarrow e^{+} e^{-}$conversions. Due to the large number of possible final states for $D$ mesons, their decay products are more difficult to eliminate directly. Instead, particles from secondary $D$ meson vertecies are removed in an iterative fit for the $B_{\text {Tag }}$ vertex, where each successive fit only considers tracks which contributed less than 6 units to the $\chi^{2}$ of the previous iteration. This process stops when either all tracks satisfy the $\chi^{2}$ requirement or only two tracks remain.

Since the beam energies, beam spot, and the momentum and decay vertex of $B_{\text {Rec }}$ are well determined, the kinematic and geometric constraints that $B_{\text {Tag }}$ originates at the beam spot with the momentum vector $p_{B_{\mathrm{Tag}}}=p_{\Upsilon(4 s)}-p_{B_{\mathrm{Rec}}}$ improves the precision of the vertex fit. Also, in order to correctly account for the correlations between the $B_{\text {Tag }}$ and $B_{\text {Rec }}$ vertices induced by these constraints, $\sigma_{\Delta z}$ is directly measured in the fit, so it reflects the errors on each track's parameters, the beam energies, and the beam spot. Tests of this algorithm on Monte Carlo events indicate that the difference 
between the true and measured values of $\Delta z$ are well described by a triple Gaussian with less than $1 \%$ in the widest component (see section 7.3 ). The events which do not lie in this Gaussian have an RMS of $190 \mu \mathrm{m}$ and the events in the smallest Gaussian have an RMS of $100 \mu \mathrm{m}$.

\section{Conversion to $\Delta t$}

The naive conversion $\Delta z=\beta \gamma c \Delta t$ provides a good estimate of the time difference between the decays of the two $B$ mesons. However, the relation

$$
\Delta z=\beta \gamma \gamma_{\operatorname{Rec}}^{*} c \Delta t+\gamma \beta_{\operatorname{Rec}}^{*} \gamma_{\operatorname{Rec}}^{*} \cos \theta_{\operatorname{Rec}}^{*} c\left\langle t_{\operatorname{Rec}}+t_{\mathrm{Tag}}\right\rangle
$$

which takes into account the $B$ momenta in the $\Upsilon(4 s)$ rest frame and the $20 \mathrm{mrad}$ rotation of the beams with respect to the $z$-axis improves, $\Delta t$ resolution by $\approx 5 \%$. Here $\beta_{\mathrm{rec}}^{*}, \gamma_{\mathrm{rec}}^{*}$, and $\theta_{\mathrm{rec}}^{*}$ respectively describe the velocity, boost, and polar angle of $B_{\text {Rec }}$ with respect to the beam axis. $\left\langle t_{\text {Rec }}+t_{\text {Tag }}\right\rangle$, which is the expected value of the sum of the decay times, is estimated by $\tau_{B}+|\Delta t|$.

\subsection{The $\Delta t$ Resolution Function}

Since the $\Delta t$ resolution is dominated by the $B_{\text {Tag }}$ vertex, it is generally insensitive to the final state of the fully reconstructed $B_{\text {Rec }}$. Nonetheless, if the error on $\Delta t$ is properly calculated, it must provide a measurement of the resolution of $\Delta t$ in every event, consequently reflecting any differences between decays. We may then expect to be able to describe the $\Delta t$ resolution for all $B_{\text {Rec }}$ final states with single function of $\Delta t$ and $\sigma_{\Delta t}$.

As illustrated in figure 7.4a, studies of simulated events indicate that the measured

$\sigma_{\Delta t}$ is directly proportional to the RMS of $\Delta t$ in simulated events. Therefore $\sigma_{\Delta t}$ is 

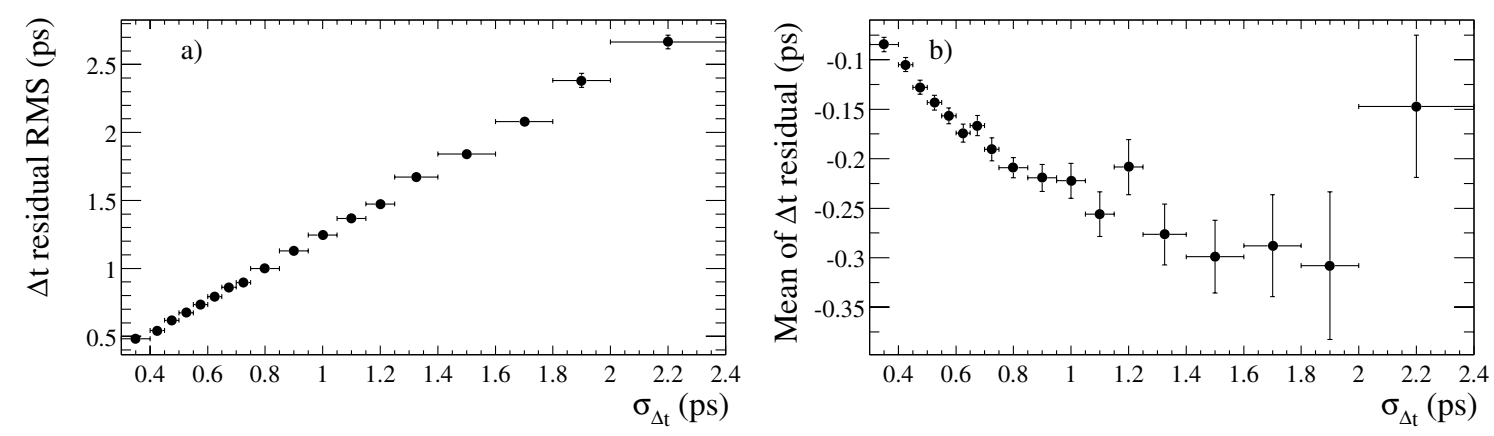

Figure 7.4: (a) the RMS spread and (b) the mean of the residual $\delta_{t}=\Delta t_{\text {meas }}-\Delta t_{\text {true }}$ versus the measured $\sigma_{\Delta t}$ in simulated $B$ decays.

indeed a measure of the $\sigma_{\Delta t}$ resolution. In fact, the difference $\delta_{t}=t_{\text {meas }}-t_{\text {true }}$ between the measured and true $\Delta t$ is well described by the sum of three Gaussian,

$$
\begin{gathered}
\mathcal{R}\left(\delta_{t}, \sigma_{\Delta_{t}} ; \hat{v}_{i}\right)=\sum_{k}^{\text {core,tail }} \frac{f_{k}}{S_{k} \sigma_{\Delta t} \sqrt{2 \pi}} \exp \left(-\frac{\left(\delta_{t}-b_{k}^{i} \sigma_{\Delta t}\right)^{2}}{2\left(S_{k} \sigma_{\Delta t}\right)^{2}}\right)+ \\
\frac{f_{\text {outl }}}{\sigma_{\text {outl }} \sqrt{2 \pi}} \exp \left(-\frac{\delta_{t}^{2}}{2 \sigma_{\text {outl }}^{2}}\right),
\end{gathered}
$$

with descending fractions of events $f_{\text {core }}, f_{\text {tail }}$, and $f_{\text {outlier }}$, and outlier width $\sigma_{\text {outl }}=$ 8 ps. The parameters, $\hat{v}_{i}$, are

- the fractions $f_{k}$,

- the scale factors $S_{k}$, and

- the scaled biases $b_{k}^{i}$.

Note that the widths of the core and tail Gaussians are scaled by with the measured $\sigma_{\Delta t}$ for each event, taking advantage of this error's estimate of the $\Delta t$ resolution. Under ideal conditions, $S_{k}$, which corresponds to the slope in figure 7.4a, would be 1 . This resolution function also provides a shift in the means of the core and tail Gaussians to account for any bias from secondary vertices of charm decays (i.e. D 
mesons) due to residual tracks which are not removed by the iterative procedure. The size of this bias is different depending on the direction of the $D$ meson. A $D$ meson traveling parallel (as opposed to perpendicular) to the beam axis pulls harder on the $z$ coordinate of the $B_{\text {Tag }}$ vertex. As figure $7.4 \mathrm{~b}$ illustrates, this bias is proportional to the $\Delta t$ resolution. Therefore in order to better estimate the bias for each $\Delta t$ measurement, the resolution function (7.7) exploits this correlation and scales the mean of the core and tail Gaussians by $\sigma_{\Delta t}$. Finally, since $b$-flavor tagging separates events based on the signatures of a specific set of $B$ decays, the charm content of the final state depends on the tagging category. Therefore, a different bias is used for each tagging category.

\subsection{The Self-tagged $B_{\text {Rec }}$ Sample}

Table 7.1 lists the seven self-tagging $B^{0}$ decays which are fully reconstructed to comprise the fully $B_{\text {Rec }}$ sample. This section summarizes the selection of these $B$ meson candidates, their parameterization in $m_{\mathrm{ES}}$ and $\Delta t$, and the measurement of the tagging performance and $\Delta t$ resolution function.

\subsubsection{Selection}

In events with at least 3 good tracks and a visible energy $\mathcal{W}>4.5 \mathrm{GeV}$, the decays are built in reverse from the final state tracks and neutrals, to the light intermediate particles, to the heavier charmed mesons, and finally the $B$ meson. The reconstruction procedure was described in section 7.2.1. Table 7.2 summarizes the lightest mesons, their reconstructed decay modes, and the selections performed on them.

$\bar{D}^{0}$ or $D^{-}$mesons are reconstructed in the decay modes listed in 7.3 with very 


\begin{tabular}{lc}
\hline \hline Decay mode & Branching fraction $(\%)$ \\
\hline$B^{0} \rightarrow D^{*-} \pi^{+}$ & $0.28 \pm 0.02$ \\
$B^{0} \rightarrow D^{*-} \rho^{+}$ & $0.73 \pm 0.15$ \\
$B^{0} \rightarrow D^{*-} a_{1}^{+}$ & $1.30 \pm 0.27$ \\
$B^{0} \rightarrow D^{-} \pi^{+}$ & $0.30 \pm 0.04$ \\
$B^{0} \rightarrow D^{-} \rho^{+}$ & $0.78 \pm 0.14$ \\
$B^{0} \rightarrow D^{-} a_{1}^{+}$ & $0.60 \pm 0.33$ \\
\hline \hline
\end{tabular}

Table 7.1: The measured branching fraction of the fully reconstructed self tagging $B$ decays in the $B_{\text {Rec }}$ sample.

\begin{tabular}{|c|c|c|c|c|c|c|}
\hline \multirow[t]{2}{*}{ Particle } & \multirow{2}{*}{$\begin{array}{l}\text { Final } \\
\text { State }\end{array}$} & \multirow{2}{*}{$\begin{array}{c}\text { Nominal } \\
\text { Mass }\left(\mathrm{MeV} / c^{2}\right)\end{array}$} & \multirow{2}{*}{$\begin{array}{c}\text { Measure } \\
\text { Width }\left(\mathrm{MeV} / \mathrm{c}^{2}\right)\end{array}$} & \multicolumn{2}{|c|}{ Mass Cut $\left(\mathrm{MeV} / \mathrm{c}^{2}\right)$} & \multirow[t]{2}{*}{ Other Selections } \\
\hline & & & & Low & High & \\
\hline$\pi^{+}$ & $\mathrm{N} / \mathrm{A}$ & 140 & $\mathrm{~N} / \mathrm{A}$ & & $\mathrm{N} / \mathrm{A}$ & Good Track (see sec 4.2 .1 ) \\
\hline$K^{+}$ & $\mathrm{N} / \mathrm{A}$ & 494 & $\mathrm{~N} / \mathrm{A}$ & & $\mathrm{N} / \mathrm{A}$ & Good Track (see sec 4.2.1) \\
\hline$\pi^{0}$ & $\gamma \gamma$ & 135 & 6.9 & 115 & 155 & $E_{\gamma}>200 \mathrm{MeV}$ \\
\hline$K_{s}^{0}$ & $\pi^{+} \pi^{-}$ & 498 & 3.2 & 462 & 534 & Vertex probability $>.1 \%$ \\
\hline$\rho^{+}$ & $\pi^{0} \pi^{+}$ & 767 & $150^{*}$ & 617 & 917 & $E_{\pi^{0}}>300 \mathrm{MeV}$ \\
\hline$a_{1}^{+}$ & $\pi^{+} \pi^{-} \pi^{+}$ & 1230 & $250-600^{*}$ & 1000 & 1600 & Vertex probability $>.1 \%$ \\
\hline
\end{tabular}

Table 7.2: Particles with $u, d$, and/or $s$ quarks used in the reconstruction of $B$ mesons.

Composite particles rely on those above them. * designates a Breit-Wigner width. 


\begin{tabular}{cccc}
\hline \hline Meson & Mass $\left(\mathrm{MeV} / c^{2}\right)$ & Decay mode & Branching fraction $(\%)$ \\
\hline $\bar{D}^{0}$ & 1865 & $K^{+} \pi^{-}$ & $3.80 \pm 0.09$ \\
& $K^{+} \rho^{-}$ & $10.2 \pm 0.9$ \\
& $K^{+} \pi^{-} \pi^{+} \pi^{-}$ & $7.5 \pm 0.3$ \\
& $K_{S}^{0} \pi^{+} \pi^{-}$ & $3.0 \pm 0.2$ \\
\hline \multirow{2}{*}{$D^{-}$} & \multirow{2}{*}{1869} & $K^{+} \pi^{-} \pi^{+}$ & $9.1 \pm 0.6$ \\
& & $K_{S}^{0} \pi^{-}$ & $1.4 \pm 0.1$ \\
\hline \hline
\end{tabular}

Table 7.3: Selected decay modes of $\bar{D}^{0}$ and $D^{-}$mesons and their branching fractions.

loose particle identification requirements ${ }^{2}$ on charge kaons and cuts on the $\pi^{0}$ mass and $\mathrm{CM}$ momentum $120<m_{\pi^{0}}<150 \mathrm{GeV} / c^{2}$ and $p_{\pi^{0}}^{*}>400 \mathrm{MeV} / c$. The $D$ candidates are selected when their reconstructed mass, $m$, satisfies $\left(m-m_{0}\right) / \sigma_{m}<3$, where $m_{0}$ is the nominal mass and $\sigma_{m}$ is the error on the mass calculated from the measured error matrices of parameters of the charged tracks. In addition, requiring that the CM momenta of these $D$ mesons are consistent with originating from a $B$, i.e. $p_{D}^{*}<2.5 \mathrm{GeV}$, reduces $e^{+} e^{-} \rightarrow c \bar{c}$ contributions and requiring $p_{D}^{*}>1.3 \mathrm{GeV}$ lowers combinatoric backgrounds. For the $\bar{D}^{0} \rightarrow K^{+} \rho^{-}$decay where background are large due to the wide $\rho$ mass, an additional cut of $\left|\cos \theta_{D \pi}\right|>0.4$, where $\theta_{D \pi}$ is the angle between the direction of the $D$ and the $\pi^{-}$from the $\rho^{-}$, exploits angular momentum conservation to reduce random combinations by $40 \%$.

${ }^{2}$ For tracks in momentum ranges, $p<.5 \mathrm{GeV} / c, .5<p<.6 \mathrm{GeV} / c$, and $p>.6 \mathrm{GeV} / c$, likelihoods from SVT and DCH, DCH only, and DIRC only (respectively) are used to select kaons. Pions and protons are rejected with requirements $L_{K} / L_{\pi}<r$ and $L_{K} / L_{p}<r$, where $r=.1$ for $p<.5 \mathrm{GeV} / c$ and $r=1$ otherwise. Kaon efficiency is flat for $p>1 \mathrm{GeV} / c$ at $96 \%$, with pion mis-id of no more than $30 \%$. 
$D^{*-}$ mesons are reconstructed in the $\bar{D}^{0} \pi^{-}$decay, which constitutes $68 \%$ of its branching fraction, using tracks with momenta $70<p<450 \mathrm{MeV} / c$ and constraints from the beam spot to improve the $D^{*-}$ vertex. Candidates are selected using the requirement that the mass difference $m\left(D^{*-}\right)-m\left(\bar{D}^{0}\right)$ is less than 2.5 standard deviations from its nominal value of $145.4 \mathrm{MeV} / c^{2}$.

\begin{tabular}{clcc}
\hline \hline$B$ Mode & $D^{0}$ mode & $\left|\cos \theta_{T}\right|$ & Other \\
\hline$D^{*-} \pi^{+}$ & $K^{+} \pi^{-}$ & - & $p_{\pi}, p_{K}>200 \mathrm{MeV} / c$ \\
& $K^{+} \pi^{-} \pi^{0}$ & - & $p_{\pi}, p_{K}>200 \mathrm{MeV} / c$ \\
& $K^{+} \pi^{-} \pi^{+} \pi^{-}$ & - & $p_{\pi}, p_{K}>200 \mathrm{MeV} / c$ \\
& $K_{S}^{0} \pi^{-} \pi^{+}$ & - & $p_{\pi}, p_{K_{S}^{0}}>200 \mathrm{MeV} / c$ \\
\hline$D^{*-} \rho^{+} \pi^{-}$ & $<0.9$ & $p_{\pi}, p_{K}>200 \mathrm{MeV} / c$ \\
& $K^{+} \pi^{-} \pi^{0}$ & $<0.9$ & $p_{\pi}, p_{K}>200 \mathrm{MeV} / c$ \\
$K^{+} \pi^{-} \pi^{+} \pi^{-}$ & $<0.8$ & $p_{\pi}, p_{K}>200 \mathrm{MeV} / c$ \\
$K_{S}^{0} \pi^{-} \pi^{+}$ & $<0.8$ & $p_{\pi}, p_{K_{S}^{0}}>200 \mathrm{MeV} / c$ \\
\hline$K^{+} \pi^{-}$ & $<0.8$ & $p_{\pi}, p_{K}>150 \mathrm{MeV} / c$ \\
$K^{+} \pi^{-} \pi^{0}$ & $<0.8$ & $p_{\pi}, p_{K}>150 \mathrm{MeV} / c$ \\
$K^{+} \pi^{-} \pi^{+} \pi^{-}$ & $<0.7$ & $p_{\pi}, p_{K}>150 \mathrm{MeV} / c$ \\
$K_{S}^{0} \pi^{-} \pi^{+}$ & $<0.7$ & $p_{\pi}>150 \mathrm{MeV} / c$ \\
& & $p_{K_{S}^{0}}>200 \mathrm{MeV} / c$ \\
\hline \hline
\end{tabular}

Table 7.4: Selection criteria for $B^{0} \rightarrow D^{*-} \pi^{+} / \rho^{+} / a_{1}^{+}$decays.

Finally the $B^{0}$ mesons are built by combining a $D^{*-}$ or $D^{-}$with a $\pi^{+}, \rho^{+}$, or $a_{1}^{+}$. Various levels of continuum and combinatoric backgrounds for every decay mode necessitate different cuts on the pion/kaon momenta and the event shape variable 


\begin{tabular}{cccc}
\hline \hline B Mode & $D^{-}$mode & $\left|\cos \theta_{T}\right|$ & Other requirements \\
\hline$D^{-} \pi^{+}$ & $K_{S}^{0} \pi^{-}$ & $<0.9$ & $p_{\pi}, p_{K}>200 \mathrm{MeV} / c$ \\
& $K^{+} \pi^{-} \pi^{+}$ & $<0.9$ & $p_{\pi}, p_{K_{S}^{0}}>200 \mathrm{MeV} / c$ \\
\hline$D^{-} \rho^{+}$ & $K_{S}^{0} \pi^{-}$ & $<0.8$ & $p_{\pi}, p_{K}>200 \mathrm{MeV} / c$ \\
& $K^{+} \pi^{-} \pi^{+}$ & $<0.8$ & $p_{\pi}, p_{K_{S}^{0}}>200 \mathrm{MeV} / c$ \\
\hline$D^{-} a_{1}^{+}$ & $K_{S}^{0} \pi^{-}$ & $<0.7$ & $p_{\pi}, p_{K}>150 \mathrm{MeV} / c$ \\
& $K^{+} \pi^{-} \pi^{+}$ & $<0.7$ & $p_{\pi}>150 \mathrm{MeV} / c$ \\
\hline \hline
\end{tabular}

Table 7.5: Selection criteria for $B^{0} \rightarrow D^{-} \pi^{+} / \rho^{+} / a_{1}^{+}$decays.

$\left|\cos \theta_{T}\right|$. Tables 7.4 and 7.5 summarize these selections. For the $B_{\text {Rec }}$ sample, $B$ candidates are retained when $|\Delta E|<3 \sigma_{\Delta E}$, where $\sigma_{\Delta E}$ is the measured $\Delta E$ resolution. Table 7.6 lists the signal yields, purities ${ }^{3}$, and $m_{\mathrm{ES}}$ and $\Delta E$ resolutions for the selected $B_{\text {Rec }}$ candidates. The $m_{\mathrm{ES}}$ distribution of all events which satisfy this $\Delta E$ requirement is shown in figure 7.5 .

\subsubsection{The Composition}

The signal decays in the $B_{\text {Rec }}$ sample are significantly more pure than those in the sample of $B^{0} \rightarrow h^{+} h^{-}$candidates, and therefore do not warrant the complication of a Fisher discriminant. In analogy to the $B^{0} \rightarrow h^{+} h^{\prime-}$ case, though the $B_{\text {Rec }}$ backgrounds may originate from multiple sources $m_{\mathrm{ES}}$ and $\Delta t$ allow the identification of three types of events: signal $B$ decays, peaking backgrounds, and combinatoric backgrounds.

\footnotetext{
${ }^{3}$ Purity is defined as the ratio of signal to all events under the signal peak.
} 


\begin{tabular}{lcccc}
\hline \hline Mode & $N_{\text {sig }}$ & $\mathcal{P}(\%)$ & $\sigma_{m_{\mathrm{ES}}}\left(\mathrm{MeV} / c^{2}\right)$ & $\sigma_{\Delta E}(\mathrm{MeV})$ \\
\hline$B^{0} \rightarrow D^{*-} \pi^{+}$ & $7333 \pm 143$ & 92 & $2.69 \pm 0.06$ & $19.2 \pm 0.3$ \\
$B^{0} \rightarrow D^{*-} \rho^{+}$ & $4668 \pm 199$ & 85 & $3.11 \pm 0.13$ & $31.4 \pm 1.2$ \\
$B^{0} \rightarrow D^{*-} a_{1}^{+}$ & $3471 \pm 150$ & 79 & $2.69 \pm 0.10$ & $13.1 \pm 0.4$ \\
\hline$B^{0} \rightarrow D^{-} \pi^{+}$ & $8222 \pm 205$ & 82 & $2.62 \pm 0.06$ & $18.2 \pm 0.3$ \\
$B^{0} \rightarrow D^{-} \rho^{+}$ & $4669 \pm 201$ & 77 & $3.00 \pm 0.12$ & $31.8 \pm 1.1$ \\
$B^{0} \rightarrow D^{-} a_{1}^{+}$ & $2634 \pm 156$ & 66 & $2.58 \pm 0.14$ & $12.6 \pm 0.4$ \\
\hline \hline
\end{tabular}

Table 7.6: Signal yield $N_{\text {sig }}$, purity $\mathcal{P}, \Delta E$ resolution $\sigma_{\Delta E}$, and $m_{\mathrm{ES}}$ resolution $\sigma_{m_{\mathrm{ES}}}$ for all reconstructed $B^{0}$ flavor eigenstates.

\section{Signal $B$ decays}

Properly reconstructed $B$ decays in the $B_{\text {Rec }}$ sample exhibit the familiar peak in $m_{\mathrm{ES}}$ which is centered about the $B$ meson mass. Meanwhile, their $\Delta t$ distribution is described by the time evolution of the mixed and unmixed $B$ meson pairs (equation 7.2), corrected for $b$-flavor tagging imperfections ( $P_{i}$ in equation 7.3), and convoluted with the $\Delta t$ resolution function $\left(\mathcal{R}_{i}\right.$ in equation 7.7$)$. Therefore the likelihood to be a signal event is

$P_{\mathrm{sig}}\left(m_{\mathrm{ES}}, \Delta t, \sigma_{\Delta t}, R, T, i\right)=G\left(m_{\mathrm{ES}} ; \mu_{m_{\mathrm{ES}}}^{i}, \sigma_{m_{\mathrm{ES}}}^{i}\right) \cdot P\left(\Delta t_{\text {true }}, R, T ; \hat{q}_{i}^{\mathrm{sig}}\right) \otimes \mathcal{R}\left(\delta_{t}, \sigma_{\Delta t} ; \hat{v}_{i}^{\mathrm{sig}}\right)$

where $R$ is the flavor of the reconstructed $B, T$ is the flavor of the tagged $B, i$ is the tagging category, $G\left(m_{\mathrm{ES}} ; \mu_{m_{\mathrm{ES}}}^{i}, \sigma_{m_{\mathrm{ES}}}^{i}\right)$ is a Gaussian describing the $m_{\mathrm{ES}}$ distribution, and $\hat{q}_{i}^{\text {sig }}$ and $\hat{v}_{i}^{\text {sig }}$ are the signal tagging and vertexing parameters. 


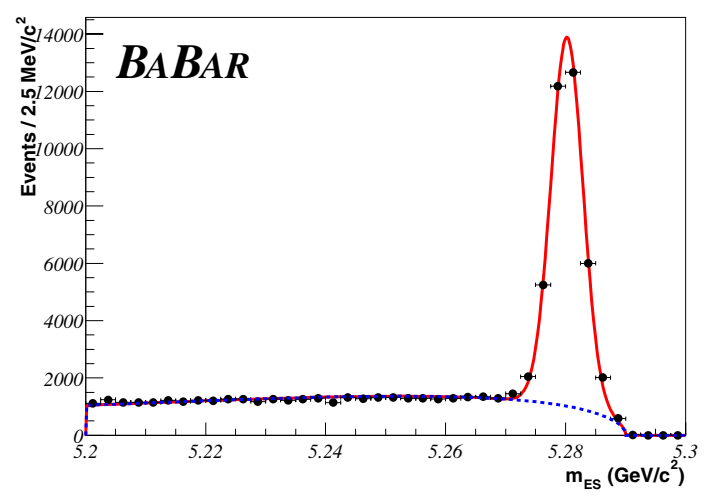

Figure 7.5: Distribution of $m_{\mathrm{ES}}$ for selected $B^{0}$ candidates the $B_{\mathrm{Rec}}$ sample.

\section{Peaking backgrounds}

Mis-reconstructed $B$ decays generally peak at the same $m_{\mathrm{ES}}$ value as the signal $B^{0}$ decays. Those candidates which originate from a real neutral $B$ decay have the same $\Delta t$ structure as the signal and are therefore properly accounted for in the signal likelihood. However when the origin of the $B^{0}$ candidate is a $B^{ \pm}$meson the timestructure is different. Under the assumption that this "peaking background" has the same $m_{\mathrm{ES}}$ distribution as the signal, the likelihood of obtaining such an event is

$$
\begin{aligned}
P_{\text {peak }}\left(m_{\mathrm{ES}}, \Delta t, \sigma_{\Delta t}, R, T, i\right)= & G\left(m_{\mathrm{ES}} ; \mu_{m_{\mathrm{ES}}}^{i}, \sigma_{m_{\mathrm{ES}}}^{i}\right) \times \\
& \frac{\bar{\epsilon}_{i}^{t}}{4 \tau_{B^{+}}} e^{\mid \Delta t_{\text {true }} / \tau_{B^{+}}}\left(1-R T D_{i}^{B+}\right) \otimes \mathcal{R}\left(\delta_{t}, \sigma_{\Delta t} ; \hat{v}_{i}^{\mathrm{sig}}\right),
\end{aligned}
$$

where the time structure reflects the expectation from a $B^{ \pm}$meson with a lifetime $\tau_{B^{+}}$and pseudo dilutions $D_{i}^{B+}$ which account for the possibility of an apparent flavor correlation between the reconstructed and tagged flavors. Note that since the $m_{\mathrm{ES}}$ PDF for this background is the same as the signal, it is only distinguished from the signal in $\Delta t$. 


\section{Combinatoric backgrounds}

The presence of events in the side-band region $m_{\mathrm{ES}}<5.26 \mathrm{GeV} / c^{2}$ is the signature of backgrounds made of random combinations of tracks from variety of possible sources. In $m_{\mathrm{ES}}$ these events are well described by the ARGUS function. However, since the origins of these events are not identifiable, two possible behaviors are empirically considered in $\Delta t$ :

- backgrounds with no lifetime:

$$
P_{\tau=0}\left(\Delta t, \sigma_{\Delta t}, R, T, i\right)=\mathcal{A}\left(m_{\mathrm{ES}} ; \xi_{i}^{\mathrm{comb}}\right) \frac{\epsilon_{i}^{\mathrm{bkg}}}{2}\left(1-R T D_{i}^{\tau=0}\right) \delta\left(\Delta t_{\mathrm{true}}\right) \otimes \mathcal{R}\left(\delta t ; \hat{v}^{\mathrm{bkg}}\right)
$$

- backgrounds with an effective lifetime $\tau_{\text {bkg }}$ :

$$
P_{\tau \neq 0}\left(\Delta t, \sigma_{\Delta t}, R, T, i\right)=\mathcal{A}\left(m_{\mathrm{ES}} ; \xi_{i}^{\mathrm{comb}}\right) \frac{\epsilon_{i}^{\mathrm{bkg}}}{4 \tau_{\mathrm{bkg}}}\left(1-R T D_{i}^{\tau \neq 0}\right) \mathrm{e}^{-\left|\Delta t_{\text {true }}\right| / \tau_{\mathrm{bkg}}} \otimes \mathcal{R}\left(\delta t ; \hat{v}^{\mathrm{bkg}}\right) .
$$

Here $\mathcal{A}$ is the ARGUS function and $\epsilon_{i}^{\mathrm{bkg}}$ are the background tagging efficiencies in category $i$. The $\Delta t$ resolution function for these events is described by different parameters $\hat{v}^{\text {bkg }}$ than the signal. Specifically, the fraction in the tail Gaussian is set to 0 , and a common bias is used for all tagging categories.

\subsubsection{Fit}

The likelihood for the $B_{\operatorname{Rec}}$ sample is constructed from the individual likelihoods for the signal and background species discussed in the previous section. Its expression is

$$
\begin{aligned}
L_{B_{\mathrm{Rec}}}\left(\hat{q}_{i}^{\mathrm{sig}}, \hat{v}^{\mathrm{sig}}, \hat{a}_{i}^{\mathrm{sig}}, \hat{v}^{\mathrm{bkg}}, \hat{q}^{\mathrm{bkg}}, \hat{a}_{i}^{\mathrm{bkg}}\right) & = \\
\frac{e^{-\left(N_{\mathrm{Rec}}^{S}+N_{\mathrm{Rec}}^{B}\right)}}{N_{\mathrm{Rec}}^{T}} & \prod_{i}^{N_{\text {Rec }}^{T}}\left\{N_{\text {Rec }}^{S}\left(\left(1-f_{\text {peak }}\right) P_{\text {sig }}\left(x_{j}\right)+f_{\text {peak }} P_{\text {peak }}\left(x_{j}\right)\right)+\right. \\
& \left.N_{\operatorname{Rec}}^{B}\left(f_{\tau=0} P_{\tau=0}\left(x_{j}\right)+\left(1-f_{\tau=0}\right) P_{\tau \neq 0}\left(x_{j}\right)\right)\right\},
\end{aligned}
$$


where the product is over the $N_{\text {Rec }}^{T}$ events in the $B_{\text {Rec }}$ sample with $x_{j}=\left\{m_{\mathrm{ES}}\right.$, $\left.\Delta t, \sigma_{\Delta t}, R, T, i\right\}_{j}$ representing the measured quantities for event $j$. The likelihood parameters and their fitted values on the $B_{\text {Rec }}$ sample are summarized in ${ }^{4}$ :

- table 7.7 - the signal $\Delta t$ resolution function parameters, $\hat{v}_{i}^{\mathrm{sig}}$,

- table 7.8- the signal tagging parameters, $\hat{q}_{i}^{\text {sig }}$,

- table 7.9- the signal yield and $m_{\mathrm{ES}}$ parameters, $\hat{a}_{i}^{\mathrm{sig}}$,

- table 7.10- the background $\Delta t$ resolution function parameters, $\hat{v}^{\mathrm{bkg}}$,

- table 7.11 - the background tagging parameters, $\hat{q}_{i}^{\mathrm{bkg}}$, and

- table 7.12- the background yield and $m_{\mathrm{ES}}$ parameters, $\hat{a}_{i}^{\mathrm{bkg}}$.

\footnotetext{
${ }^{4}$ In order to save space, we list the results obtained from the simultaneous fit to the $B_{\text {Rec }}$ and $B^{0} \rightarrow h^{+} h^{-}$samples. The values of these parameters are dominated by the $B_{\text {rec }}$ sample which contains $\approx 50 \times$ more signal $B$ decays than the $B^{0} \rightarrow h^{+} h^{-}$sample. Therefore fits to only the $B_{\text {rec }}$ candidates yield nearly identical results.
} 


\begin{tabular}{lccl}
\hline \hline Parameter & Value & Error & Description \\
\hline$f_{\text {tail }}$ & $0.92208 \cdot 10^{-1}$ & $0.20332 \cdot 10^{-1}$ & Tail Fraction \\
$f_{\text {outl }}$ & $0.40541 \cdot 10^{-2}$ & $0.12248 \cdot 10^{-2}$ & Outlier Fraction \\
$b_{\text {core }}^{1}$ & $0.43728 \cdot 10^{-1}$ & $0.62087 \cdot 10^{-1}$ & Cat. 1 core bias \\
$b_{\text {core }}^{2}$ & -0.22345 & $0.50391 \cdot 10^{-1}$ & Cat. 2 core bias \\
$b_{\text {core }}^{3}$ & -0.22217 & $0.43899 \cdot 10^{-1}$ & Cat. 3 core bias \\
$b_{\text {core }}^{4}$ & -0.18565 & $0.45151 \cdot 10^{-1}$ & Cat. 4 core bias \\
$b_{\text {core }}^{5}$ & -0.18527 & $0.33962 \cdot 10^{-1}$ & Cat. 5 core bias \\
$S_{\text {core }}$ & 1.1063 & $0.42013 \cdot 10^{-1}$ & Core scale factor \\
$S_{\text {tail }}$ & 3.0000 & fixed & Tail scale factor \\
$b_{\text {tail }}$ & -1.5898 & 0.38907 & Tail offset \\
\hline \hline
\end{tabular}

Table 7.7: $\Delta t$ resolution function parameters extracted from the simultaneous fit to the $B_{\text {Rec }}$ and $B^{0} \rightarrow h^{+} h^{-}$samples. 


\begin{tabular}{lccl}
\hline \hline Parameter & Value & Error & Description \\
\hline $\bar{\epsilon}_{1}^{t}$ & $0.92037 \cdot 10^{-1}$ & $0.17286 \cdot 10^{-2}$ & Cat. 1 tagging eff. \\
$\bar{\epsilon}_{2}^{t}$ & 0.16576 & $0.23569 \cdot 10^{-2}$ & Cat. 2 tagging eff. \\
$\bar{\epsilon}_{3}^{t}$ & 0.19780 & $0.25767 \cdot 10^{-2}$ & Cat. 3 tagging eff. \\
$\bar{\epsilon}_{4}^{t}$ & 0.20110 & $0.26034 \cdot 10^{-2}$ & Cat. 4 tagging eff. \\
$D_{1}$ & 0.93720 & $0.13137 \cdot 10^{-1}$ & Cat. 1 dilution \\
$D_{2}$ & 0.80729 & $0.14518 \cdot 10^{-1}$ & Cat. 2 dilution \\
$D_{3}$ & 0.58765 & $0.16326 \cdot 10^{-1}$ & Cat. 3 dilution \\
$D_{4}$ & 0.36206 & $0.17742 \cdot 10^{-1}$ & Cat. 4 dilution \\
$\Delta D_{1}$ & $0.32885 \cdot 10^{-1}$ & $0.26870 \cdot 10^{-1}$ & Cat. 1 dilution diff. \\
$\Delta D_{2}$ & $0.60220 \cdot 10^{-1}$ & $0.28273 \cdot 10^{-1}$ & Cat. 2 dilution diff. \\
$\Delta D_{3}$ & 0.10799 & $0.28710 \cdot 10^{-1}$ & Cat. 3 dilution diff. \\
$\Delta D_{4}$ & $0.64346 \cdot 10^{-1}$ & $0.28304 \cdot 10^{-1}$ & Cat. 4 dilution diff. \\
$\mu_{1}$ & $0.54642 \cdot 10^{-2}$ & $0.31257 \cdot 10^{-1}$ & Cat. 1 tag eff. asym. \\
$\mu_{2}$ & $-0.58309 \cdot 10^{-1}$ & $0.27804 \cdot 10^{-1}$ & Cat. 2 tag eff. asym. \\
$\mu_{3}$ & $-0.26496 \cdot 10^{-1}$ & $0.28900 \cdot 10^{-1}$ & Cat. 3 tag eff. asym. \\
$\mu_{4}$ & $0.32557 \cdot 10^{-1}$ & $0.30199 \cdot 10^{-1}$ & Cat. 4 tag eff. asym. \\
\hline \hline
\end{tabular}

Table 7.8: Tagging parameters extracted from the simultaneous fit to the $B_{\text {Rec }}$ and $B^{0} \rightarrow h^{+} h^{-}$samples. See 7.1.1 for the definition of the categories. 


\begin{tabular}{lccl}
\hline \hline Parameter & Value & Error & Description \\
\hline$N_{\mathrm{Rec}}^{S}$ & 30319. & 198.78 & Signal yield \\
$\mu_{m_{\mathrm{ES}}}^{1}$ & 5.2803 & $0.52734 \cdot 10^{-4}$ & Cat. $1 m_{\mathrm{ES}}$ mean \\
$\mu_{m_{\mathrm{ES}}}^{2}$ & 5.2802 & $0.42817 \cdot 10^{-4}$ & Cat. $2 m_{\mathrm{ES}}$ mean \\
$\mu_{m_{\mathrm{ES}}}^{3}$ & 5.2802 & $0.40193 \cdot 10^{-4}$ & Cat. $3 m_{\mathrm{ES}}$ mean \\
$\mu_{m_{\mathrm{ES}}}^{4}$ & 5.2802 & $0.39549 \cdot 10^{-4}$ & Cat. $4 m_{\mathrm{ES}}$ mean \\
$\mu_{m_{\mathrm{ES}}}^{5}$ & 5.2802 & $0.32210 \cdot 10^{-4}$ & Cat. $5 m_{\mathrm{ES}}$ mean \\
$\sigma_{m_{\mathrm{ES}}}^{1}$ & $0.26219 \cdot 10^{-2}$ & $0.43288 \cdot 10^{-4}$ & Cat. $1 m_{\mathrm{ES}}$ res. \\
$\sigma_{m_{\mathrm{ES}}}^{2}$ & $0.26638 \cdot 10^{-2}$ & $0.37005 \cdot 10^{-4}$ & Cat. $2 m_{\mathrm{ES}}$ res. \\
$\sigma_{m_{\mathrm{ES}}}^{3}$ & $0.26601 \cdot 10^{-2}$ & $0.35736 \cdot 10^{-4}$ & Cat. $3 m_{\mathrm{ES}}$ res. \\
$\sigma_{m_{\mathrm{ES}}}^{4}$ & $0.26286 \cdot 10^{-2}$ & $0.36533 \cdot 10^{-4}$ & Cat. $4 m_{\mathrm{ES}}$ res. \\
$\sigma_{m_{\mathrm{ES}}^{5}}$ & $0.27134 \cdot 10^{-2}$ & $0.28393 \cdot 10^{-4}$ & Cat. $5 m_{\mathrm{ES}}$ res. \\
$\nu_{1}$ & $-0.22885 \cdot 10^{-1}$ & $0.27642 \cdot 10^{-1}$ & Cat. $1 \mathrm{reco}$. eff. asym. \\
$\nu_{2}$ & $-0.18824 \cdot 10^{-1}$ & $0.22703 \cdot 10^{-1}$ & Cat. 2 reco. eff. asym. \\
$\nu_{3}$ & $-0.15312 \cdot 10^{-1}$ & $0.22488 \cdot 10^{-1}$ & Cat. 3 reco. eff. asym. \\
$\nu_{4}$ & $-0.45361 \cdot 10^{-1}$ & $0.23044 \cdot 10^{-1}$ & Cat. $4 \mathrm{reco}$ eff. asym. \\
$\nu_{5}$ & $-0.27252 \cdot 10^{-2}$ & $0.10678 \cdot 10^{-1}$ & Cat. 5 reco. eff. asym. \\
\hline \hline
\end{tabular}

Table 7.9: Signal yield and $m_{\mathrm{ES}}$ parameters extracted from the simultaneous fit to the $B_{\text {Rec }}$ and $B^{0} \rightarrow h^{+} h^{-}$samples. 


\begin{tabular}{lccl}
\hline \hline Parameter & Value & Error & Description \\
\hline$\tau_{\text {bkg }}$ & 1.2983 & $0.37362 \cdot 10^{-1}$ & Peaking bkg lifetime \\
$S_{\text {core }}^{\text {bkg }}$ & 1.3400 & $0.12250 \cdot 10^{-1}$ & Comb. bkg. core scale factor \\
$b_{\text {core }}^{\text {bkg }}$ & $-0.26661 \cdot 10^{-1}$ & $0.92543 \cdot 10^{-2}$ & Comb. bkg. core mean \\
$f_{\text {outl }}^{\text {bkg }}$ & $0.17470 \cdot 10^{-1}$ & $0.14810 \cdot 10^{-2}$ & Comb. bkg. outlier fraction \\
\hline \hline
\end{tabular}

Table 7.10: Background $\Delta t$ parameters extracted from the simultaneous fit to the $B_{\text {Rec }}$ and $B^{0} \rightarrow h^{+} h^{--}$samples.

\begin{tabular}{lccl}
\hline \hline Parameter & Value & Error & Description \\
\hline$D_{1}^{B^{+}}$ & 0.91010 & fixed & Cat. 1 peaking bkg dilution \\
$D_{2}^{B^{+}}$ & 0.75100 & fixed & Cat. 2 peaking bkg dilution \\
$D_{3}^{B^{+}}$ & 0.62110 & fixed & Cat. 3 peaking bkg dilution \\
$D_{4}^{B^{+}}$ & 0.29880 & fixed & Cat. 4 peaking bkg dilution \\
$D_{1}^{\tau=0}$ & 1.3820 & 0.33421 & Cat. $1 \tau=0$ bkg dilution \\
$D_{2}^{\tau=0}$ & 0.66647 & $0.32446 \cdot 10^{-1}$ & Cat. $2 \tau=0$ bkg dilution \\
$D_{3}^{\tau=0}$ & 0.39005 & $0.24889 \cdot 10^{-1}$ & Cat. $3 \tau=0$ bkg dilution \\
$D_{4}^{\tau=0}$ & 0.16270 & $0.24869 \cdot 10^{-1}$ & Cat. $4 \tau=0$ bkg dilution \\
$D_{1}^{\tau \neq 0}$ & 0.20403 & 0.12234 & Cat. $1 \tau \neq 0$ bkg dilution \\
$D_{2}^{\tau \neq 0}$ & 0.26428 & $0.45905 \cdot 10^{-1}$ & Cat. $2 \tau \neq 0$ bkg dilution \\
$D_{3}^{\tau \neq 0}$ & 0.29215 & $0.40363 \cdot 10^{-1}$ & Cat. $3 \tau \neq 0$ bkg dilution \\
$D_{4}^{\tau \neq 0}$ & $0.35997 \cdot 10^{-1}$ & $0.44136 \cdot 10^{-1}$ & Cat. $4 \tau \neq 0$ bkg dilution \\
\hline \hline
\end{tabular}

Table 7.11: Background tagging parameters extracted from the simultaneous fit to the $B_{\text {Rec }}$ and $B^{0} \rightarrow h^{+} h^{-}$samples. 


\begin{tabular}{|c|c|c|c|}
\hline Parameter & Value & Error & Description \\
\hline$N_{\operatorname{Rec}}^{B}$ & 38409 . & 218.19 & Bkg yield \\
\hline$\xi_{1}^{\mathrm{comb}}$ & 67.751 & 6.9167 & Cat. 1 ARGUS func. $\xi$ \\
\hline$\xi_{2}^{\text {comb }}$ & 33.149 & 1.9819 & Cat. 2 ARGUS func. $\xi$ \\
\hline$\xi_{3}^{\text {comb }}$ & 30.565 & 1.5538 & Cat. 3 ARGUS func. $\xi$ \\
\hline$\xi_{4}^{\mathrm{comb}}$ & 33.446 & 1.5733 & Cat. 4 ARGUS func. $\xi$ \\
\hline$\xi_{5}^{\text {comb }}$ & 30.472 & 0.98651 & Cat. 5 ARGUS func. $\xi$ \\
\hline$\epsilon_{1}^{\mathrm{bkg}}$ & $0.12000 \cdot 10^{-1}$ & $0.68685 \cdot 10^{-3}$ & Cat. 1 bkg. tag eff. \\
\hline$\epsilon_{2}^{\mathrm{bkg}}$ & 0.12139 & $0.18601 \cdot 10^{-2}$ & Cat. 2 bkg. tag eff. \\
\hline$\epsilon_{3}^{\mathrm{bkg}}$ & 0.19580 & $0.22495 \cdot 10^{-2}$ & Cat. 3 bkg. tag eff. \\
\hline$\epsilon_{4}^{\mathrm{bkg}}$ & 0.19382 & $0.22530 \cdot 10^{-2}$ & Cat. 4 bkg. tag eff. \\
\hline$f_{\text {outl }}^{\text {bkg }}$ & $0.17470 \cdot 10^{-1}$ & $0.14810 \cdot 10^{-2}$ & Comb. bkg. outlier fraction \\
\hline$f_{\text {peak }}$ & $0.15000 \cdot 10^{-1}$ & fixed & Peaking bkg fraction \\
\hline$f_{\tau=0}^{1}$ & 0.26854 & $0.64440 \cdot 10^{-1}$ & Cat. $1 \tau=0$ comb. bkg. fraction \\
\hline$f_{\tau=0}^{2}$ & 0.59764 & $0.22346 \cdot 10^{-1}$ & Cat. $2 \tau=0$ comb. bkg. fraction \\
\hline$f_{\tau=0}^{3}$ & 0.63249 & $0.19361 \cdot 10^{-1}$ & Cat. $3 \tau=0$ comb. bkg. fraction \\
\hline$f_{\tau=0}^{4}$ & 0.65991 & $0.18374 \cdot 10^{-1}$ & Cat. $4 \tau=0$ comb. bkg. fraction \\
\hline$f_{\tau=0}^{5}$ & 0.71467 & $0.14294 \cdot 10^{-1}$ & Cat. $5 \tau=0$ comb. bkg. fraction \\
\hline
\end{tabular}

Table 7.12: Background yield and $m_{\mathrm{ES}}$ parameters extracted from the simultaneous fit to the $B_{\text {Rec }}$ and $B^{0} \rightarrow h^{+} h^{-}$samples. 


\section{Chapter 8}

\section{Analysis of the Time-dependent}

\section{Asymmetries}

After BABAR's first measurement of the $B^{0} \rightarrow h^{+} h^{\prime-}$ branching fractions in the summer of 2000, attention shifted to developing an analysis of the time-dependent CP asymmetries in $B^{0} \rightarrow \pi^{+} \pi^{-}$. This task required the merger of elements of two distinct analyses: the $b$-flavor tagging/ $\Delta t$ measurement techniques, described in the previous chapter, and the extraction of rare charmless signals, described in chapter 6 . The most natural course of progression suggested mimicking the two steps of the $\sin 2 \beta$ analysis, where the signal events are first isolated and then passed to an independent fit which extracted the $\mathrm{CP}$ asymmetries. Ultimately this procedure proved not to be optimal for the $B^{0} \rightarrow \pi^{+} \pi^{-}$decays, which have significantly smaller braching fractions than the $B$ decays to charmonium final states and suffer from much larger backgrounds from both continuum and $B$ decays $\left(\right.$ i.e. $\left.B^{0} \rightarrow K^{+} \pi^{-}\right)$.

Several arguments led to the development of a ML fit to the whole $B^{0} \rightarrow h^{+} h^{\prime-}$ sample which included background processes, rather than a subset enriched in $B^{0} \rightarrow$ $\pi^{+} \pi^{-}$decays. The most compelling reason for such a fit was that it guaranteed obtaining the most statistically significant result while also facilitated the accounting of 

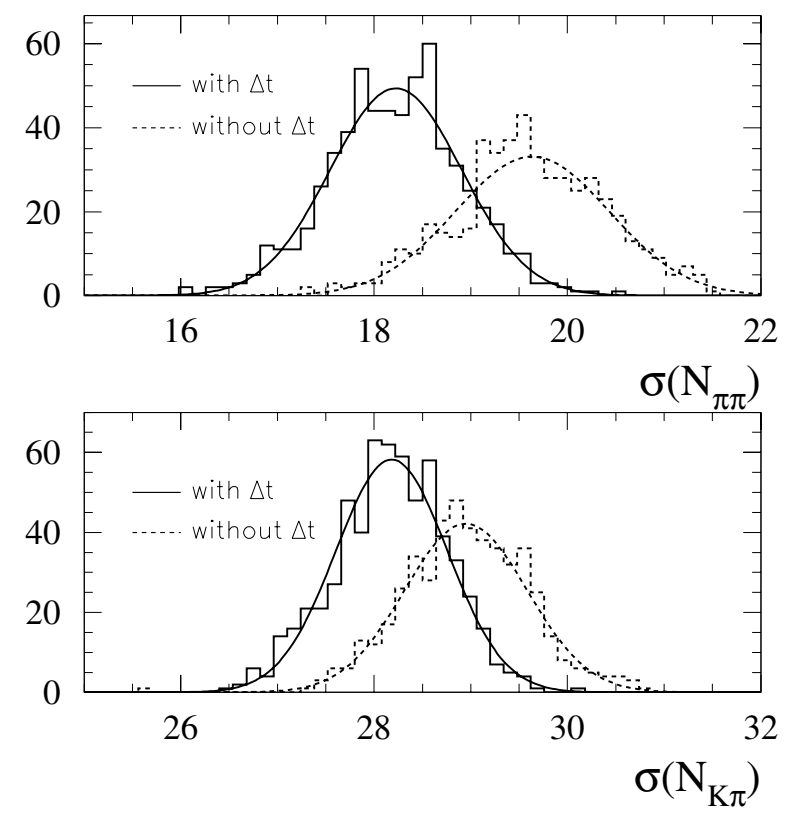

Figure 8.1: The measured error on $B^{0} \rightarrow \pi^{+} \pi^{-}$and $B^{0} \rightarrow K^{+} \pi^{-}$yields from fits to toy Monte Carlo experiments, with (solid) and without (dashed) $\Delta t$ in the ML fit.

systematic errors due to the parameterizations of the backgrounds (see appendix A). Another argument for fitting the full $B^{0} \rightarrow h^{+} h^{--}$sample centered about the virtues of extracting all branching fractions and $\mathrm{CP}$ asymmetries simultaneously. With the addition of $\Delta t$ and flavor tagging, the ML fit, aware of the shorter effective lifetime (section 8.2.1) and lower tagging efficiency (section 8.2.2) of the continuum events, better separates signal and background events, resulting in gains in the statistical precision of fitted yields. For example, figure 8.1 illustrates how the errors on the yields improve when $\Delta t$ is added to the likelihood. Indeed, this particular effect had been advertised as a possible advantage of rare branching fraction analysis at the asymmetric machines which are able to measure the $B$ lifetime. However, for this analysis, the small improvement in the statistical precision of the branching fractions 
$\left(\approx 1.0 \%\right.$ in $B^{0} \rightarrow \pi^{+} \pi^{-}$and $\approx 0.2 \%$ in $\left.B^{0} \rightarrow K^{+} \pi^{-}\right)$does not warrant understanding the influences of tagging and vertexing on the yield measurements such that the increase in systematic errors are smaller than the statistical gain. Therefore we choose not to extract the branching fractions from fits which include the variables necessary for the time-dependent analysis. But, in order to facilitate comparisons with the branching fraction fit, we retain the events which were not tagged in the fit, despite the fact that they do not directly influence the time-dependent measurement.

\subsection{Time Structure of Tagged $B^{0} \rightarrow h^{+} h^{\prime-}$ Decays}

Of the four $B^{0} \rightarrow h^{+} h^{\prime-}$ decays, the $\pi^{+} \pi^{-}$and $K^{+} K^{-}$final states are CP eigenstates so their time structure is described by

$$
\Gamma_{\bar{B}^{0}}^{B^{0}}(\Delta t)=\frac{e^{-\frac{|\Delta t|}{\tau}}}{4 \tau}\left(1 \pm S_{f} \sin \left(\Delta m_{d} \Delta t\right) \mp C_{f} \cos \left(\Delta m_{d} \Delta t\right)\right),
$$

where the asymmetry defined as

$$
A_{\mathrm{CP}}^{\pi \pi}(\Delta t)=\frac{\Gamma_{B^{0}}-\Gamma_{\bar{B}^{0}}}{\Gamma_{B^{0}}+\Gamma_{\bar{B}^{0}}}=S_{\pi \pi} \sin \left(\Delta m_{d} \Delta t\right)-C_{\pi \pi} \cos \left(\Delta m_{d} \Delta t\right)
$$

is between the two possible flavors of the tagged meson. Since we did not observe a significant signal from $B^{0} \rightarrow K^{+} K^{-}$decays, we fix $N_{K K}^{S}=0$ and ignore the asymmetry in this mode by setting $S_{K K}=C_{K K}=0$. Integration of these relations lead to the total number of signal $\pi^{+} \pi^{-}$events tagged as $B^{0}$ or $\bar{B}^{0}$ :

$$
\begin{aligned}
& N_{\pi \pi}^{B^{0}}=\int_{-\infty}^{\infty} \Gamma_{B^{0}} d(\Delta t)=N_{\pi \pi}\left(1-\frac{C_{\pi \pi}}{1+\left(\Delta m_{d} \tau\right)^{2}}\right) \\
& N_{\pi \pi}^{\bar{B}^{0}}=\int_{-\infty}^{\infty} \Gamma_{\bar{B}^{0}} d(\Delta t)=N_{\pi \pi}\left(1+\frac{C_{\pi \pi}}{1+\left(\Delta m_{d} \tau\right)^{2}}\right)
\end{aligned}
$$

and the time integrated $\mathrm{CP}$ asymmetry $A_{\pi \pi}=-C_{\pi \pi}$. 
Meanwhile, since the kaon charges of the $K^{+} \pi^{-}$and $K^{-} \pi^{+}$final states carry the same sign as the $b$-quark from which they originate, these decays are self-tagging and are described by

$$
\Gamma_{\text {mixed }}^{\text {unmixed }}(\Delta t)=\frac{e^{-\frac{|\Delta t|}{\tau}}}{4 \tau}\left(1 \pm \cos \left(\Delta m_{d} \Delta t\right)\right)
$$

Here the the asymmetry is between mixed and unmixed events

$$
A_{\text {mix }}^{K \pi}(\Delta t)=\frac{\Gamma_{\text {unmixed }}-\Gamma_{\text {mixed }}}{\Gamma_{\text {unmixed }}+\Gamma_{\text {mixed }}}=\cos \left(\Delta m_{d} \Delta t\right)
$$

and the total number of mixed events is

$$
N_{K \pi}^{\text {mixed }}=\left(1-\frac{1}{1+\left(\Delta m_{d} \tau\right)^{2}}\right) N_{K \pi} \approx(17 \%) N_{K \pi} .
$$

Comparison of $\Gamma_{B^{0}}$ and $\Gamma_{\bar{B}^{0}}$ for $\pi \pi$ and $K \pi$ final states demonstrates the influence of $K^{ \pm} \pi^{\mp}$ events which are mis-identified as $\pi^{+} \pi^{-}$. The $B^{0} / \bar{B}^{0}$-tagged $K \pi$ decay distributions are

$$
\begin{aligned}
\Gamma_{B^{0}}^{K \pi} & =\frac{\Gamma_{\text {mixed }} \cdot \mathcal{B}\left(B^{0} \rightarrow K^{+} \pi^{-}\right)+\Gamma_{\text {unmixed }} \cdot \mathcal{B}\left(\bar{B}^{0} \rightarrow K^{-} \pi^{+}\right)}{\mathcal{B}\left(B^{0} \rightarrow K^{+} \pi^{-}\right)+\mathcal{B}\left(\bar{B}^{0} \rightarrow K^{-} \pi^{+}\right)} \\
& =1-A_{K \pi} \cos \left(\Delta m_{d} \Delta t\right), \\
\Gamma_{\bar{B}^{0}}^{K \pi} & =\frac{\Gamma_{\text {unmixed }} \cdot \mathcal{B}\left(B^{0} \rightarrow K^{+} \pi^{-}\right)+\Gamma_{\text {mixed }} \cdot \mathcal{B}\left(\bar{B}^{0} \rightarrow K^{-} \pi^{+}\right)}{\mathcal{B}\left(B^{0} \rightarrow K^{+} \pi^{-}\right)+\mathcal{B}\left(\bar{B}^{0} \rightarrow K^{-} \pi^{+}\right)} \\
& =1+A_{K \pi} \cos \left(\Delta m_{d} \Delta t\right),
\end{aligned}
$$

leading to the time-dependent $\mathrm{CP}$ asymmetry $A_{\mathrm{CP}}^{K \pi}(\Delta t)=A_{K \pi} \cos \left(\Delta m_{d} \Delta t\right)$, where $A_{K \pi}$ is the time-integrated CP asymmetry we measured in chapter 6 . This suggests that $K \pi$ events which are mis-identified as $\pi \pi$ carry a "fake" $\pi \pi \mathrm{CP}$ asymmetry $C_{\pi \pi}=-A_{K \pi}$. The use of all kinematic and particle identification information, the proper accounting of the time-distributions, and allowing $A_{K \pi}$ to float along with $S_{\pi \pi}$ and $C_{\pi \pi}$, insures that the influence of the signal $K \pi$ background to $\pi \pi$ is reflected in the measured errors on $C_{\pi \pi}$. 
All the expressions we have discussed in this section are fundamental relations which do not account for $b$-flavor tagging and $\Delta t$ corrections. The observable relations may be calculated from the convolution of the $\Delta t$ resolution function (7.7) with equation (7.4) for $\mathrm{CP}$ events and (7.3) for mixing events.

As discussed in the previous chapter, we expect that the tagging and resolution function parameters of $B^{0} \rightarrow h^{+} h^{--}$decays are the same as the signal events in the $B_{\text {Rec }}$ sample and choose to simultaneously fit both samples in order to propagate the parameters from the latter to the former. We validate this assumption by comparing ML fits to samples of simulated $B_{\text {Rec }}$ and $B^{0} \rightarrow h^{+} h^{\prime-}$ events. As table 8.1 illustrates, the resolution function parameters extracted from $D^{(*)-} \pi^{+} / \rho^{+} / a_{1}^{+}$and $B^{0} \rightarrow \pi^{+} \pi^{-}$ Monte Carlo are consistent. For tagging, we fit $B^{0} \rightarrow D^{(*)-} \pi^{+} / \rho^{+} / a_{1}^{+}$and $B^{0} \rightarrow$ $K^{+} \pi^{-}$Monte Carlo because $B^{0} \rightarrow \pi^{+} \pi^{-}$decays are not self-tagging. Table 8.2 shows that these parameters are also consistent. We find that in all cases the $B^{0} \rightarrow$ $D^{(*)-} \pi^{+} / \rho^{+} / a_{1}^{+}$and $B^{0} \rightarrow h^{+} h^{\prime-}$ parameterizations agree well.

\subsection{Time and Flavor Structure of Background Can- didates to $B^{0} \rightarrow h^{+} h^{-}$}

As with the kinematics and event shape, we parameterize the $\Delta t$ and flavor tagging structure of the continuum backgrounds to $B^{0} \rightarrow h^{+} h^{\prime-}$ decays empirically. However, in addition to the particle identification information which allowed us to separate the background into the species $\pi^{+} \pi^{-}, K^{+} \pi^{-}, K^{-} \pi^{+}$, and $K^{+} K^{-}$for the branching fraction fit, we may now also separate these events using tagged flavor and tagging category. 


\begin{tabular}{lcc}
\hline \hline Parameter & $B^{0} \rightarrow D^{(*)-} \pi^{+} / \rho^{+} / a_{1}^{+} \mathrm{MC}$ & $B^{0} \rightarrow \pi^{+} \pi^{-} \mathrm{MC}$ \\
\hline$f_{\text {tail }}$ & $0.0952 \pm 0.0115$ & $0.1046 \pm 0.0207$ \\
$f_{\text {outl }}$ & $0.0024 \pm 0.0006$ & $0.0050 \pm 0.0009$ \\
$b_{\text {core }}^{1}$ & $-0.1067 \pm 0.0319$ & $-0.1707 \pm 0.0579$ \\
$b_{\text {core }}^{2}$ & $-0.2565 \pm 0.0265$ & $-0.2425 \pm 0.0467$ \\
$b_{\text {core }}^{3}$ & $-0.2557 \pm 0.0237$ & $-0.2563 \pm 0.0387$ \\
$b_{\text {core }}^{4}$ & $-0.2094 \pm 0.0237$ & $-0.2780 \pm 0.0387$ \\
$S_{\text {core }}$ & $1.1794 \pm 0.0258$ & $1.1785 \pm 0.0389$ \\
$b_{\text {tail }}$ & $-0.9088 \pm 0.1761$ & $-1.5157 \pm 0.3446$ \\
\hline \hline
\end{tabular}

Table 8.1: $\Delta t$ resolution function parameters extracted from simulated $B^{0} \rightarrow$ $D^{(*)-} \pi^{+} / \rho^{+} / a_{1}^{+}$and $B^{0} \rightarrow \pi^{+} \pi^{-}$events.

\subsection{1 $\Delta t$}

The quarks from $e^{+} e^{-} \rightarrow u \bar{u}, d \bar{d}, s \bar{s}$ hadronize after production, resulting in a single vertex for the event at the production point and $\Delta t_{\text {true }}=0$ for the sub-set which mimic the kinematical features of a $B$ decay. Detector resolution smears this value to produce a $\Delta t$ distribution that is narrower than that of $B \bar{B}$ events, which have a lifetime. Meanwhile the $c$-quark lifetime causes $e^{+} e^{-} \rightarrow c \bar{c}$ events to contain two vertecies, which are typically less separated than that of the longer living $B$ meson pairs. $B^{0} \rightarrow h^{+} h^{-}$candidates from $e^{+} e^{-} \rightarrow c \bar{c}$ events are usually constructed from one track from each oppositely flying jet, producing pseudo-decay vertecies for the two fake $B$ 's which are between the two real vertecies in the event. The result is a $\Delta t$ distribution which is also significantly narrower than $B$ decays, but slightly wider than the lighter quark backgrounds. Figure 8.2 plots the $\Delta t$ distributions for 


\begin{tabular}{lcc}
\hline \hline Parameter & $B^{0} \rightarrow D^{(*)-} \pi^{+} / \rho^{+} / a_{1}^{+} \mathrm{MC}$ & $B^{0} \rightarrow K^{+} \pi^{-} \mathrm{MC}$ \\
\hline $\bar{\epsilon}_{1}^{t}$ & $0.103 \pm 0.001$ & $0.110 \pm 0.001$ \\
$\bar{\epsilon}_{2}^{t}$ & $0.175 \pm 0.001$ & $0.177 \pm 0.002$ \\
$\bar{\epsilon}_{3}^{t}$ & $0.200 \pm 0.001$ & $0.195 \pm 0.002$ \\
$\bar{\epsilon}_{4}^{t}$ & $0.198 \pm 0.001$ & $0.198 \pm 0.002$ \\
$D_{1}$ & $0.9301 \pm 0.0063$ & $0.9421 \pm 0.0100$ \\
$D_{2}$ & $0.8201 \pm 0.0063$ & $0.8289 \pm 0.0105$ \\
$D_{3}$ & $0.5765 \pm 0.0075$ & $0.5780 \pm 0.0127$ \\
$D_{4}$ & $0.3815 \pm 0.0082$ & $0.3957 \pm 0.0137$ \\
$\Delta D_{1}$ & $0.0188 \pm 0.0109$ & $0.0068 \pm 0.0174$ \\
$\Delta D_{2}$ & $0.0031 \pm 0.0104$ & $-0.0011 \pm 0.0174$ \\
$\Delta D_{3}$ & $0.0531 \pm 0.0118$ & $0.0525 \pm 0.0200$ \\
$\Delta D_{4}$ & $0.0638 \pm 0.0128$ & $0.0478 \pm 0.0213$ \\
\hline \hline
\end{tabular}

Table 8.2: Tagging parameters extracted from simulated $B^{0} \rightarrow D^{(*)-} \pi^{+} / \rho^{+} / a_{1}^{+}$and $B^{0} \rightarrow K^{+} \pi^{-}$events. 
these two background sources along with signal $B^{0} \rightarrow \pi^{+} \pi^{-}$decays. Since the visible difference in the $\Delta t$ distributions of the charm and light quark events $(u \bar{u}, d \bar{d}, s \bar{s})$ is not very significant and we have no other means of distinguishing the two sources, we choose not to parameterize them separately.

There are no known physics processes which induce a CP asymmetry in the continuum backgrounds to $B^{0} \rightarrow h^{+} h^{\prime-}$ decays. As figure 8.3 illustrates, the $\Delta t$ distributions of the $B^{0}$ and $\bar{B}^{0}$ tagged candidates in the $m_{\mathrm{ES}}<5.26 \mathrm{GeV} / c^{2}$ sideband region exhibit no $\mathrm{CP}$ asymmetry $A_{C P}(\Delta t)$. As a test we measure the asymmetries $S_{\mathrm{bkg}}=-0.0648 \pm 0.06267$ and $C_{\mathrm{bkg}}=-0.0153 \pm 0.0214$, using the $B^{0} \rightarrow \pi^{+} \pi^{-} \mathrm{PDF}$ for $\Delta t$ to fit these events. Finding no $\mathrm{CP}$ asymmetry, we choose to not distinguish the flavor tag in the background $\Delta t$ parameterization. In addition, we searched for significant differences in the $\Delta t$ distributions for the different background species and different tagging categories and found none. We therefore describe all background species, flavor tags, and tagging categories by a single $\Delta t \mathrm{PDF}$.

In our studies, we consider two PDFs for the continuum $\Delta t$ distribution. The first is the sum of the convolution of an exponential with a Gaussian plus two Gaussian functions:

$$
\begin{aligned}
\mathcal{R}_{\text {bkg }}\left(\Delta t ; \bar{v}_{\text {EXP }}^{b k g}\right)= & \left(1-f_{\text {tail }}^{\mathrm{bkg}}-f_{\text {outl }}^{\mathrm{bkg}}\right)[\mathcal{E} \otimes \mathcal{G}]\left(\Delta t ; \tau^{\mathrm{bkg}}, \sigma_{\text {core }}^{\mathrm{bkg}}\right) \\
+ & f_{\text {tail }}^{\mathrm{bkg}} \mathcal{G}\left(\sigma_{\text {tail }}^{\mathrm{bkg}}, \mu_{\text {tail }}^{\mathrm{bkg}}\right)+f_{\text {outl }}^{\mathrm{bkg}} \mathcal{G}\left(\sigma_{\text {outl }}^{\mathrm{bkg}}, \mu_{\text {outl }}^{\mathrm{bkg}}\right),
\end{aligned}
$$

where $\tau^{\text {bkg }}$ may be thought of as the effective lifetime of the background, and $\sigma_{\text {outl }}^{\text {bkg }}$ and $\mu_{\text {outl }}^{\mathrm{bkg}}$ are fixed to $8 \mathrm{ps}$ and $0 \mathrm{ps}$, respectively. The second is $\mathcal{R}\left(\Delta t_{\text {true }}, \sigma_{\Delta t} ; \bar{v}_{3 G S}^{b k g}\right)$, i.e. the signal $\Delta t$ resolution function in equation (7.7) with different core and tail Gaussian fractions, biases, and scale factors. Figure 8.4 displays the result of fitting these two PDFs to the side-band region $m_{\mathrm{ES}}<5.26 \mathrm{GeV} / c^{2}$. Due to concerns regarding observed correlations between $\sigma_{\Delta t}$ and $\mathcal{F}$ and $\Delta t$ (see section 8.3), we quote the central values 


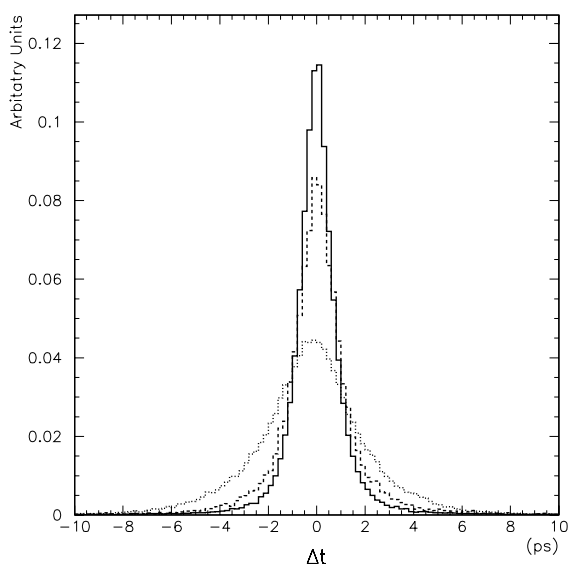

Figure 8.2: The measured $\Delta t$ of $B^{0} \rightarrow h^{+} h^{\prime-}$ candidates from (in order of increasing width) simulated $e^{+} e^{-} \rightarrow u \bar{u}, d \bar{d}, s \bar{s}, e^{+} e^{-} \rightarrow c \bar{c}$, and signal $B^{0} \rightarrow \pi^{+} \pi^{-}$events. The RMS of these distributions are $1.320 \pm 0.002 \mathrm{ps}, 1.697 \pm 0.005 \mathrm{ps}$, and $2.370 \pm 0.007 \mathrm{ps}$, respectively. All distributions are normalized to same area.
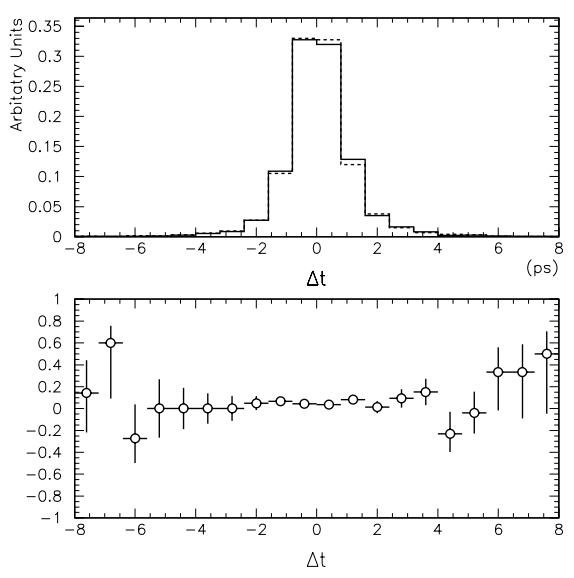

Figure 8.3: The $\Delta t$ distribution of candidate $B^{0}$ (solid) and $\bar{B}^{0}$ (dashed) $B^{0} \rightarrow h^{+} h^{\prime-}$ decays (top), and their apparent CP asymmetry (bottom), for events in the sideband region $m_{\mathrm{ES}}<5.26 \mathrm{GeV} / c^{2}$. 

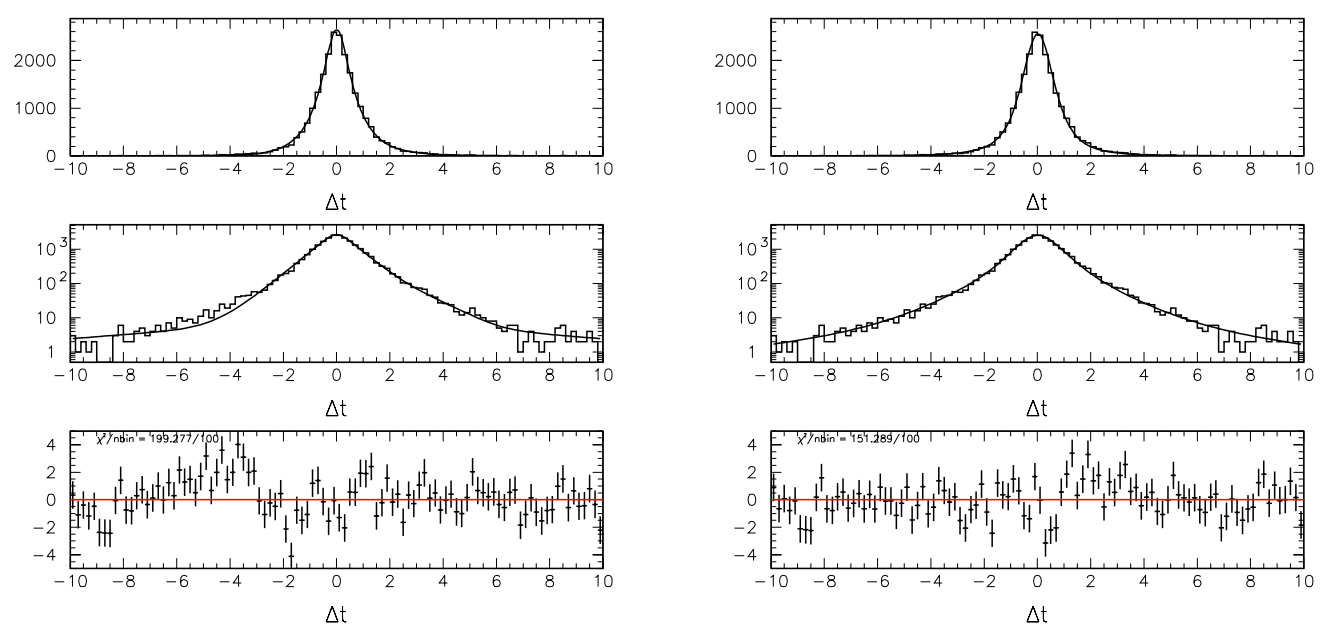

Figure 8.4: The $\Delta t$ distribution of candidates in the $B^{0} \rightarrow h^{+} h^{\prime-}$ sample in the sideband region $m_{\mathrm{ES}}<5.26 \mathrm{GeV} / c^{2}$, fitted with $\mathcal{R}_{\mathrm{bkg}}\left(\Delta t ; \bar{v}_{\mathrm{EXP}}^{b k g}\right)$ (left) and $\mathcal{R}\left(\Delta t_{\text {true }}, \sigma_{\Delta t} ; \bar{v}_{3 G S}^{b k g}\right)$ (right). The top and middle plots display the data (points) and fit results (line), on linear and log scales, respectively. The bottom plot shows the residual of the data minus the fit function.

of the measured quantities from the first PDF.

\subsubsection{Flavor Tagging}

The separation of the $B^{0} \rightarrow h^{+} h^{\prime-}$ (or $B_{\text {Rec }}$ ) sample into tagging categories creates five sub-samples, each with a different signal and background yield for every species which must be extracted in the ML fit. The choice in the previous chapter to normalize the signal $\Delta t$ PDFs to the tagging efficiencies provides a reparameterization of these yields, so that the number of events in category $i$ belonging to species $j$ is simply $N_{i}^{S, B} \epsilon_{i j}^{t}$, where $\epsilon_{i j}^{t}$ is the tagging efficiency, and the total yield of each species $\left(N_{i}^{S, B}\right)$ is directly extracted in the fit. For consistency we normalize the background $\Delta t$ PDFs 

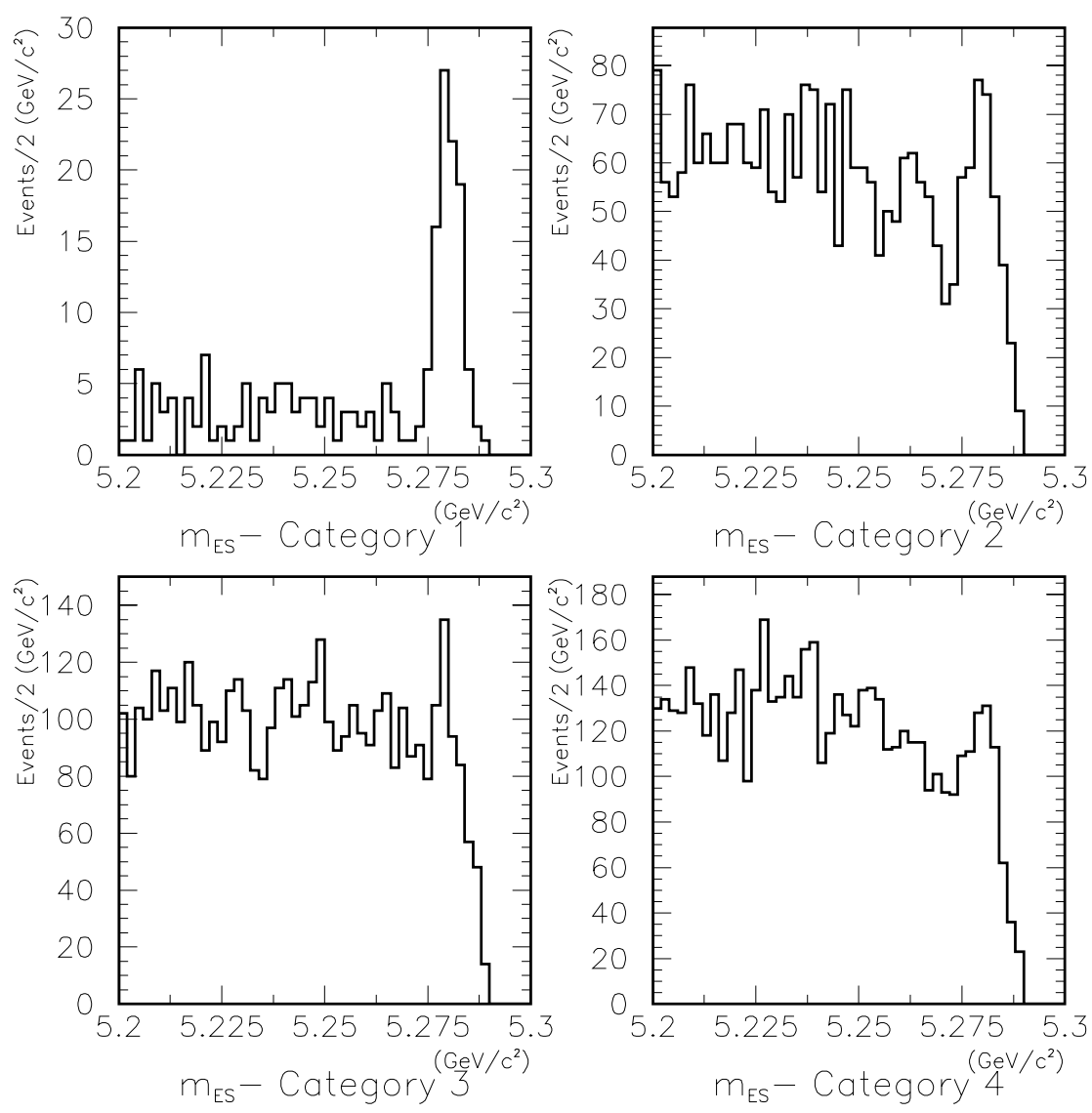

Figure 8.5: The $m_{\mathrm{ES}}$ distribution of the tagged events in the $B^{0} \rightarrow h^{+} h^{\prime-}$ sample, separated by tagging category. 
in the same manner, and rely on the fit to determine background tagging efficiencies from the sideband events in the $B^{0} \rightarrow h^{+} h^{\prime-}$ sample.

The advantage of using tagging efficiencies rather yields becomes clear when we consider the signal to background ratio in each category, visually presented in figure 8.5. Category 1, which contains the events tagged with electrons and muons, is very pure because the hadronizing $u, d, s$, and $c$ quarks of the continuum background do not produce high momentum leptons. Therefore a $B^{0} \rightarrow h^{+} h^{\prime-}$ candidate which is tagged in category 1 is significantly more likely to be signal than background. The tagging efficiencies incorporated into the the $\Delta t$ PDFs properly account for the signal purities in each category. Effectively, the tagging category becomes a discrete discriminating variable, with signal and background PDFs, which are simply the tagging efficiencies, determined by the signal $B_{\text {Rec }}$ and sideband $B^{0} \rightarrow h^{+} h^{\prime-}$ events, respectively.

We find that the background tagging efficiencies exhibit a correlation between species, flavor tag, and tag category. Kaons in continuum events are a good example of a mechanism which causes such an effect. Kaons mostly originate from $e^{+} e^{-} \rightarrow$ $s \bar{s}, c \bar{c}$, where each $q$ or $\bar{q}$ quark preferentially produces a kaon with opposite flavor than that of the anti-quark. In such an event, one kaon may be used to build a $B^{0} \rightarrow h^{+} h^{\prime-}$ candidate while the other may provide the tagged flavor. The result is that background $K^{+} \pi^{-}$candidates are more likely to reside in the kaon category than $\pi^{+} \pi^{-}$, and more likely to carry a $B^{0} \operatorname{tag}$ than $K^{-} \pi^{+}$. We account for these effects by introducing separate tagging efficiencies for each background species, tag flavor $T$, and tag category $i$ :

$$
\text { - } \pi^{+} \pi^{-}: \bar{\epsilon}_{i}^{\pi \pi}\left(1+T \mu_{i}^{\pi \pi}\right)
$$

- $K^{+} \pi^{-}: \bar{\epsilon}_{i}^{K \pi}\left(1+T \mu_{i}^{K \pi}\right)$, 
- $K^{-} \pi^{+}: \bar{\epsilon}_{i}^{K \pi}\left(1-T \mu_{i}^{K \pi}\right)$, and

- $K^{+} K^{-}: \bar{\epsilon}_{i}^{K K}\left(1+T \mu_{i}^{K K}\right)$.

Note that the $\mu_{i}^{j}$ parameterize the tagging efficiency asymmetry between $B^{0}$ and $\bar{B}^{0}$ tags for $\pi \pi$ and $K K$, and between mixed and unmixed candidates for $K \pi$.

\subsection{Correlation Studies}

The addition of $b$-flavor tagging and $\Delta t$ information to the branching fraction fit warrants an examination of any correlations between the variables used previously and those which have been newly introduced. In this section we survey the observed correlations as a means of documenting how these issues were addressed with specific features in the ML fit or Toy MC prior to performing the measurement. When practical we account for a specific correlation in the parameterizations in the fit. Otherwise we reproduce the correlation in toy Monte Carlo experiments and evaluate the effect of ignoring it in the fit. When the correlation is neither easily parameterizable in the fit nor reproducible in the toy Monte Carlo, we compare fits which include and exclude one variable in data with expectations from toy Monte Carlo.

\subsection{1 $\mathcal{F}$ and $\sigma_{\Delta t}$}

Table 8.3 lists the corresponding linear correlation coefficients determined in the same manner as in section 6.1.2. The only noteworthy entry, the correlation between $\mathcal{F}$ and $\sigma_{\Delta t}$, is due the sensitivity of both variables to the number and momenta of the tracks which were not used in the construction of each $B^{0} \rightarrow h^{+} h^{\prime-}$ candidate. Figure 8.6 plots the mean and RMS of the $\mathcal{F}$ distribution for background events as a function of $\sigma_{\Delta t}$, illustrating that the events with small $\sigma_{\Delta t}$ are on average more signal-like in 
Table 8.3: Linear correlation coefficients for the variables $\left\{m_{\mathrm{ES}}, \Delta E, \mathcal{F}, \theta_{c}^{+}, \theta_{c}^{-}, \Delta t, \sigma_{\Delta t}, \frac{\Delta t}{\sigma_{\Delta t}}\right\}$. The on-resonance sideband region is defined as $5.2<m_{\mathrm{ES}}<5.26 \mathrm{GeV} / c^{2}$.

\begin{tabular}{|c|c|c|}
\hline Variables & Sideband & $\pi \pi \mathrm{MC}$ \\
\hline$\left(m_{\mathrm{ES}}, \Delta t\right)$ & -0.01194 & 0.00190 \\
\hline$\left(m_{\mathrm{ES}}, \sigma_{\Delta t}\right)$ & -0.04032 & 0.00418 \\
\hline$\left(m_{\mathrm{ES}}, \frac{\Delta t}{\sigma_{\Delta t}}\right)$ & -0.00612 & 0.01060 \\
\hline$(\Delta E, \Delta t)$ & 0.03312 & 0.00022 \\
\hline$\left(\Delta E, \sigma_{\Delta t}\right)$ & 0.01960 & 0.00160 \\
\hline$\left(\Delta E, \frac{\Delta t}{\sigma_{\Delta t}}\right)$ & 0.02896 & -0.00168 \\
\hline$(\mathcal{F}, \Delta t)$ & -0.02121 & -0.01612 \\
\hline$\left(\mathcal{F}, \sigma_{\Delta t}\right)$ & -0.15843 & 0.03870 \\
\hline$\left(\mathcal{F}, \frac{\Delta t}{\sigma_{\Delta t}}\right)$ & -0.01836 & -0.00666 \\
\hline$\left(\theta_{c}^{+}, \Delta t\right)$ & -0.00440 & 0.01333 \\
\hline$\left(\theta_{c}^{+}, \sigma_{\Delta t}\right)$ & 0.00509 & 0.01599 \\
\hline$\left(\theta_{c}^{+}, \frac{\Delta t}{\sigma_{\Delta t}}\right)$ & -0.00297 & 0.01854 \\
\hline$\left(\theta_{c}^{-}, \Delta t\right)$ & -0.00904 & -0.02120 \\
\hline$\left(\theta_{c}^{-}, \sigma_{\Delta t}\right)$ & 0.00153 & 0.00109 \\
\hline$\left(\theta_{c}^{-}, \frac{\Delta t}{\sigma_{\Delta t}}\right)$ & -0.00900 & -0.01584 \\
\hline$\left(\Delta t, \sigma_{\Delta t}\right)$ & 0.08751 & -0.03951 \\
\hline
\end{tabular}



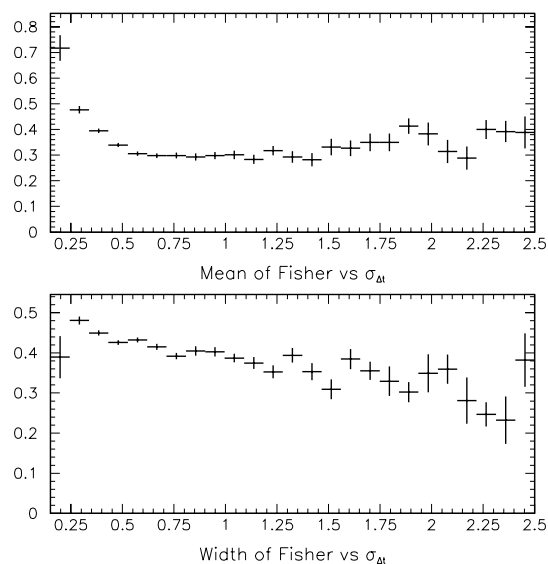

Figure 8.6: Mean (top) and RMS (bottom) of $\mathcal{F}$ versus the error on $\Delta t$.

$\mathcal{F}$. Several factors led us to assume that the influence of this correlations on the measurement of $S_{\pi \pi}$ and $C_{\pi \pi}$ is negligible. Specifically,

- the correlation is only $\approx 16 \%$,

- $\sigma_{\Delta t}$ is not used in the description of background events, and

- the signal's dependence on $\sigma_{\Delta t}$ is only in the resolution function.

In section 8.6.2 we validate this assumption by confirming that the difference in the CP asymmetries when $\mathcal{F}$ is included and excluded from the ML fit is consistent with the expected change of statistical precision.

\subsection{2 $\mathcal{F}$ and Tagging Category}

As figure 8.7 illustrates, we observe that the separation between signal and background in $\mathcal{F}$ is better for the tagging categories with high $Q$. Accounting for this difference between the categories requires the addition of 20 floating parameters for 

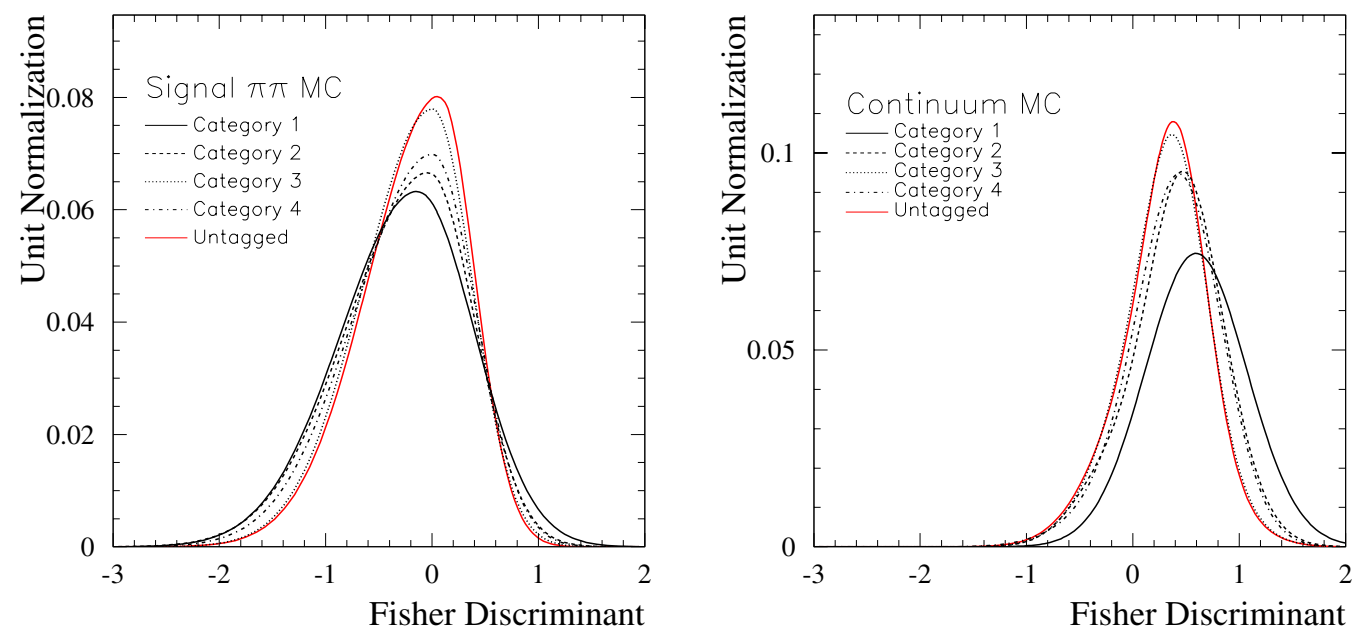

Figure 8.7: Fisher PDFs for signal (left) and $q \bar{q}$ (right) separated by tagging category.

background and 12 parameters for signal to the ML fit. In order to avoid the complication of multiple $\mathcal{F}$ PDFs, we choose to use the average. To evaluate the impact of this decision, we generate the distributions in figure 8.7 in toy Monte Carlo studies. We find that fitting with category averaged PDFs produces no bias in the measurement of $S_{\pi \pi}$ or $C_{\pi \pi}$.

\subsection{3 $\Delta t$ and $\sigma_{\Delta t}$ in Background}

Figure 8.8 shows that the mean and RMS of the $\Delta t$ distribution of candidates in the sideband region $m_{\mathrm{ES}}<5.26 \mathrm{GeV} / c^{2}$, exhibit a dependence on $\sigma(\Delta t)$. The underlying source of this correlation is the $B$ meson boost correction and beam axis correction in the $\Delta z$ to $\Delta t$ conversion in equation (7.5). These corrections have no meaning in the continuum background events and are purely artificial. Though future versions of this analysis will likely use $\Delta z$, which does not suffer from this effect, to parameterize the time-structure of the background, the analysis presented in this dissertation relies 

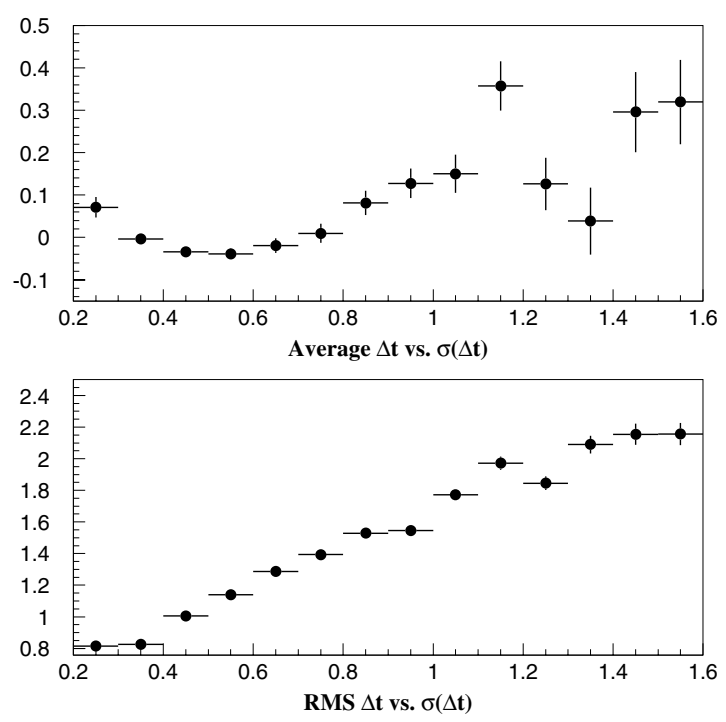

Figure 8.8: Projection plots of the mean and RMS of $\Delta t$ as a function of $\sigma(\Delta t)$ from the on-resonance $m_{\mathrm{ES}}$ sideband sample.

on $\Delta t$. However, we reproduce the effect of the $\Delta z$ to $\Delta t$ conversion in our nominal toy Monte Carlos, and we find no bias in the measurement $S_{\pi \pi}$ and $C_{\pi \pi}$.

\subsection{4 $\sigma_{\Delta t}$ and Tagging Category}

The distributions of $\Delta t$ error for both signal and background candidates are slightly different for each tagging category. However, no parameterization of the $\sigma_{\Delta t}$ shape is explicitly employed in the ML fit. Once again we rely on the toy Monte Carlo to assess the impact of this correlation by generating different $\sigma_{\Delta t}$ distributions for each tagging category for both signal and background and find no bias. 


\subsubsection{Background $m_{\mathrm{ES}}$ and Tagging Category}

We observe that continuum $m_{\mathrm{ES}}$ shape is dependent on the tagging category. We therefore allow different ARGUS function parameters $\xi_{i}$ for each category $i$. The values of these parameters, extracted from the full ML fit, are listed in table 8.5.

\subsection{Maximum Likelihood Fit}

We fit the time-dependent asymmetries in $B^{0} \rightarrow \pi^{+} \pi^{-}$in a simultaneous fit to the $B^{0} \rightarrow h^{+} h^{\prime-}$ and $B_{\text {Rec }}$ samples, extracting a total of 119 parameters, including

- the yield in each $B^{0} \rightarrow h^{+} h^{--}$species (table 8.4- 8 fitted parameters),

- the parameterization of the $\Delta t$ resolution function (table 7.7- 9 fitted parameters),

- the parameterization of the signal tagging performance (table 7.8- 16 fitted parameters),

- the descriptions of the $m_{\mathrm{ES}}, \Delta E$, and $\mathcal{F}$ shapes of the $B^{0} \rightarrow h^{+} h^{\prime-}$ background (table 8.513 fitted parameters),

- the $B^{0} \rightarrow h^{+} h^{\prime-}$ background tagging efficiencies (table 8.6- 12 fitted parameters) and tagging efficiency asymmetries (table 8.7- 12 fitted parameters),

- the description of the $\Delta t$ shape for the $B^{0} \rightarrow h^{+} h^{\prime-}$ background (table $8.8-6$ fitted parameters),

- the yield of $B_{\mathrm{Rec}}$ signal events and their parameterization in $m_{\mathrm{ES}}$ (table 7.9 - 16 fitted parameters), 
- the parameters describing the $B_{\text {Rec }}$ background in $\Delta t$ (table $7.10-4$ fitted parameters),

- the tagging parameters for the $B_{\text {Rec }}$ background (table $7.11-8$ fitted parameters), and

- the yield of $B_{\text {Rec }}$ background events and their $m_{\mathrm{ES}}$ parameters (table $7.12-16$ fitted parameters).

These tables list the final fitted values of the parameters from the full fit which also measures the asymmetries $S_{\pi \pi}$ and $C_{\pi \pi}$. As in the branching fraction analysis discussed in chapter 6 , we chose to determine and freeze every aspect of the analysis while blind to the measured values of the asymmetries. In order to be able to perform full fits to the data before examining $S_{\pi \pi}$ and $C_{\pi \pi}$, we obscured their measured values by applying a separate random sign change and shift to each quantity. Therefore, while performing blind studies, the asymmetries reported by the fit are

$$
\begin{aligned}
& S_{\pi \pi}^{\text {Blind }}= \pm S_{\pi \pi}^{\text {True }}+S_{\pi \pi}^{\mathrm{Off}} \\
& C_{\pi \pi}^{\text {Blind }}= \pm C_{\pi \pi}^{\text {True }}+C_{\pi \pi}^{\mathrm{Off}},
\end{aligned}
$$

where $S_{\pi \pi}^{\text {True }}$ and $C_{\pi \pi}^{\text {True }}$ are the asymmetries internally supplied to the signal PDFs by the fit, and the \pm signs, $S_{\pi \pi}^{\text {Off }}$, and $C_{\pi \pi}^{\text {Off }}$ are picked by a deterministic random algorithm based on an input key. In order to remain blind to unphysical fit results, which is possible in light of the large expected errors on the measured asymmetries, we choose the acceptable range for the $S_{\pi \pi}^{\text {Off }}$ and $C_{\pi \pi}^{\text {Off }}$ offsets to be between -5 and 5 . 
Table 8.4: Summary of $B^{0} \rightarrow h^{+} h^{--}$yields in the CP maximum likelihood fit.

\begin{tabular}{lccl}
\hline \hline Parameter & Value & Error & Description \\
\hline$N_{\pi \pi}^{S}$ & 154.46 & 17.377 & signal $\pi \pi$ events \\
$N_{K \pi}^{S}$ & 603.99 & 28.728 & signal $K \pi$ events \\
$\mathcal{A}_{K \pi}^{S}$ & $-0.99605 \cdot 10^{-1}$ & $0.47389 \cdot 10^{-1}$ & signal $K^{ \pm} \pi^{\mp}$ charge asym. \\
$N_{K K}^{S}$ & 0.0000 & fixed & signal $K K$ events \\
$N_{\pi \pi}^{B}$ & 12191. & 116.25 & bkg. $\pi \pi$ events \\
$N_{K \pi}^{B}$ & 7974.0 & 101.23 & bkg. $K \pi$ events \\
$\mathcal{A}_{K \pi}^{B}$ & $0.12586 \cdot 10^{-1}$ & $0.12566 \cdot 10^{-1}$ & bkg. $K^{ \pm} \pi^{\mp}$ charge asym. \\
$N_{K K}^{B}$ & 5146.9 & 76.760 & bkg. $K K$ events \\
\hline \hline
\end{tabular}

\subsection{Validations}

Before unblinding the results, we validate that the complicated fit which we have constructed properly extracts $S_{\pi \pi}$ and $C_{\pi \pi}$. In addition to the toy and Geant4 Monte Carlo tests which were available to us for the branching fraction analysis, we may also use the $B^{0} \rightarrow K^{+} \pi^{-}$events in our sample to confirm that the time-structure and flavor tags of $B$ decays have been properly accounted for in the fit.

\subsubsection{Toy Monte Carlo}

We generate toy Monte Carlo experiments using the parameters extracted from the blind fit to the full $B^{0} \rightarrow h^{+} h^{\prime-}$ sample (see section 8.4). For the time-structure of events, we rely on algorithms which first simulate the underlying physics and then smear the results to reproduce the measured $\Delta t$ resolution and tagging dilutions, 
Table 8.5: Summary of $B^{0} \rightarrow h^{+} h^{-}$background $m_{\mathrm{ES}}, \Delta E$, and $\mathcal{F}$ parameters in the CP maximum likelihood fit.

\begin{tabular}{lccl}
\hline \hline Parameter & Value & Error & Description \\
\hline$\xi_{1}$ & 22.662 & 10.761 & bkg. $m_{\mathrm{ES}}$ ARGUS param. in cat. 1 \\
$\xi_{2}$ & 18.988 & 2.2888 & bkg. $m_{\mathrm{ES}}$ ARGUS param. in cat. 2 \\
$\xi_{3}$ & 26.336 & 1.7391 & bkg. $m_{\mathrm{ES}}$ ARGUS param. in cat. 3 \\
$\xi_{4}$ & 21.077 & 1.5575 & bkg. $m_{\mathrm{ES}}$ ARGUS param. in cat. 4 \\
$\xi_{5}$ & 21.507 & 0.97583 & bkg. $m_{\mathrm{ES}}$ ARGUS param. in cat. 5 \\
$\Delta E_{p 1}$ & -0.74419 & $0.73426 \cdot 10^{-1}$ & bkg. $\Delta E$ linear term \\
$\Delta E_{p 2}$ & 0.67151 & 0.95063 & bkg. $\Delta E$ quadratic term \\
$\mathcal{F}_{f_{1}}$ & 0.86594 & $0.20233 \cdot 10^{-1}$ & bkg. Fisher fraction of first Gaussian \\
$\mathcal{F}_{\mu_{1}}$ & 0.36373 & $0.46050 \cdot 10^{-2}$ & bkg. Fisher mean of first Gaussian \\
$\mathcal{F}_{\sigma_{1}}$ & 0.38332 & $0.47418 \cdot 10^{-2}$ & bkg. Fisher width of first Gaussian \\
$\mathcal{F}_{\mu_{2}}$ & $0.92302 \cdot 10^{-1}$ & $0.28783 \cdot 10^{-1}$ & bkg. Fisher mean of second Gaussian \\
$\mathcal{F}_{\sigma_{2}}$ & 0.65322 & $0.19546 \cdot 10^{-1}$ & bkg. Fisher width of second Gaussian \\
\hline \hline
\end{tabular}


Table 8.6: Summary of $B^{0} \rightarrow h^{+} h^{\prime-}$ background tagging efficiencies in the CP maximum likelihood fit.

\begin{tabular}{|c|c|c|c|}
\hline Parameter & Value & Error & Description \\
\hline $\bar{\epsilon}_{1}^{\pi \pi}$ & $0.49425 \cdot 10^{-2}$ & $0.67441 \cdot 10^{-3}$ & Cat $1 \pi \pi$ bkg. tag. eff. \\
\hline $\bar{\epsilon}_{2}^{\pi \pi}$ & $0.86002 \cdot 10^{-1}$ & $0.26804 \cdot 10^{-2}$ & Cat $2 \pi \pi$ bkg. tag. eff. \\
\hline $\bar{\epsilon}_{3}^{\pi \pi}$ & 0.15559 & $0.34736 \cdot 10^{-2}$ & Cat $3 \pi \pi$ bkg. tag. eff. \\
\hline $\bar{\epsilon}_{4}^{\pi \pi}$ & 0.21164 & $0.38869 \cdot 10^{-2}$ & Cat $4 \pi \pi$ bkg. tag. eff. \\
\hline $\bar{\epsilon}_{1}^{K \pi}$ & $0.31830 \cdot 10^{-2}$ & $0.76804 \cdot 10^{-3}$ & Cat $1 K \pi$ bkg. tag. eff. \\
\hline $\bar{\epsilon}_{2}^{K \pi}$ & 0.12530 & $0.41046 \cdot 10^{-2}$ & Cat $2 K \pi$ bkg. tag. eff. \\
\hline $\bar{\epsilon}_{3}^{K \pi}$ & 0.19557 & $0.49963 \cdot 10^{-2}$ & Cat $3 K \pi$ bkg. tag. eff. \\
\hline $\bar{\epsilon}_{4}^{K \pi}$ & 0.19128 & $0.50170 \cdot 10^{-2}$ & Cat $4 K \pi$ bkg. tag. eff. \\
\hline $\bar{\epsilon}_{1}^{K K}$ & $0.60731 \cdot 10^{-2}$ & $0.11518 \cdot 10^{-2}$ & Cat $1 K K$ bkg. tag. eff. \\
\hline $\bar{\epsilon}_{2}^{K K}$ & $0.78240 \cdot 10^{-1}$ & $0.40500 \cdot 10^{-2}$ & Cat $2 K K$ bkg. tag. eff. \\
\hline $\bar{\epsilon}_{3}^{K K}$ & 0.14343 & $0.52913 \cdot 10^{-2}$ & Cat $3 K K$ bkg. tag. eff. \\
\hline $\bar{\epsilon}_{4}^{K K}$ & 0.22097 & $0.61763 \cdot 10^{-2}$ & Cat $4 K K$ bkg. tag. eff. \\
\hline
\end{tabular}


Table 8.7: Summary of $B^{0} \rightarrow h^{+} h^{-}$background tagging asymmetries in the CP maximum likelihood fit.

\begin{tabular}{lccl}
\hline \hline Parameter & Value & Error & Description \\
\hline$\mu_{1}^{\pi \pi}$ & 0.20314 & 0.13201 & Cat $1 \pi \pi$ bkg. tag. asym. \\
$\mu_{2}^{\pi \pi}$ & $-0.72759 \cdot 10^{-2}$ & $0.32418 \cdot 10^{-1}$ & Cat $2 \pi \pi$ bkg. tag. asym. \\
$\mu_{3}^{\pi \pi}$ & $0.52518 \cdot 10^{-1}$ & $0.24117 \cdot 10^{-1}$ & Cat $3 \pi \pi$ bkg. tag. asym. \\
$\mu_{4}^{\pi \pi}$ & $0.78856 \cdot 10^{-1}$ & $0.20468 \cdot 10^{-1}$ & Cat $4 \pi \pi$ bkg. tag. asym. \\
$\mu_{1}^{K \pi}$ & $0.99517 \cdot 10^{-1}$ & 0.23733 & Cat $1 K \pi$ bkg. tag. asym. \\
$\mu_{2}^{K \pi}$ & -0.69524 & $0.26340 \cdot 10^{-1}$ & Cat $2 K \pi$ bkg. tag. asym. \\
$\mu_{3}^{K \pi}$ & -0.43122 & $0.25904 \cdot 10^{-1}$ & Cat $3 K \pi$ bkg. tag. asym. \\
$\mu_{4}^{K \pi}$ & -0.18246 & $0.28508 \cdot 10^{-1}$ & Cat $4 K \pi$ bkg. tag. asym. \\
$\mu_{1}^{K K}$ & $0.43547 \cdot 10^{-1}$ & 0.18754 & Cat $1 K K$ bkg. tag. asym. \\
$\mu_{2}^{K K}$ & $-0.53178 \cdot 10^{-2}$ & $0.53629 \cdot 10^{-1}$ & Cat $2 K K$ bkg. tag. asym. \\
$\mu_{3}^{K K}$ & $0.80033 \cdot 10^{-1}$ & $0.39518 \cdot 10^{-1}$ & Cat $3 K K$ bkg. tag. asym. \\
$\mu_{4}^{K K}$ & $0.64678 \cdot 10^{-1}$ & $0.31345 \cdot 10^{-1}$ & Cat $4 K K$ bkg. tag. asym. \\
\hline \hline
\end{tabular}


Table 8.8: Summary of $B^{0} \rightarrow h^{+} h^{-}$background $\Delta t$ parameters in the CP maximum likelihood fit.

\begin{tabular}{lccl}
\hline \hline Parameter & Value & Error & Description \\
\hline$\tau^{\text {bkg }}$ & 0.68128 & $0.13414 \cdot 10^{-1}$ & bkg. effective lifetime \\
$\sigma_{\text {core }}^{\text {bkg }}$ & 0.22672 & $0.16934 \cdot 10^{-1}$ & bkg. Core width \\
$f_{\text {tail }}^{\text {bkg }}$ & $0.20960 \cdot 10^{-1}$ & $0.15740 \cdot 10^{-1}$ & bkg. Tail fraction \\
$\mu_{\text {tail }}^{\text {bkg }}$ & 2.2487 & 1.1752 & bkg. Tail mean \\
$\sigma_{\text {tail }}^{\text {bkg }}$ & 1.6065 & 0.35732 & bkg. Tail width \\
$f_{\text {outl }}^{\text {bkg }}$ & $0.21910 \cdot 10^{-1}$ & $0.15521 \cdot 10^{-2}$ & bkg. Out fraction \\
$\mu_{\text {outl }}^{\text {bkg }}$ & 0.0000 & fixed & bkg. Out mean \\
$\sigma_{\text {outl }}^{\text {bkg }}$ & 8.0000 & fixed & bkg. Out width \\
\hline \hline
\end{tabular}

dilution differences, efficiencies, and efficiency asymmetries. We find this approach more rigorous than generating events directly from the PDFs, since it explicitly tests the fit's consistency with the resolution and tagging performance models. We also reproduce the effects discussed in section 8.3. Specifically we include:

- Different $\sigma_{\Delta t}$ distributions for each category for signal.

- Proper background $\Delta t$ and $\sigma_{\Delta t}$ modeling, using

- different $\sigma_{\Delta z}$ distributions for each category for background,

- parameterization of $\Delta z / \sigma_{\Delta z}$, and

- $\left(\Delta z, \sigma_{\Delta z}\right) \rightarrow\left(\Delta t, \sigma_{\Delta t}\right)$ conversion using the kinematics of the background events. 

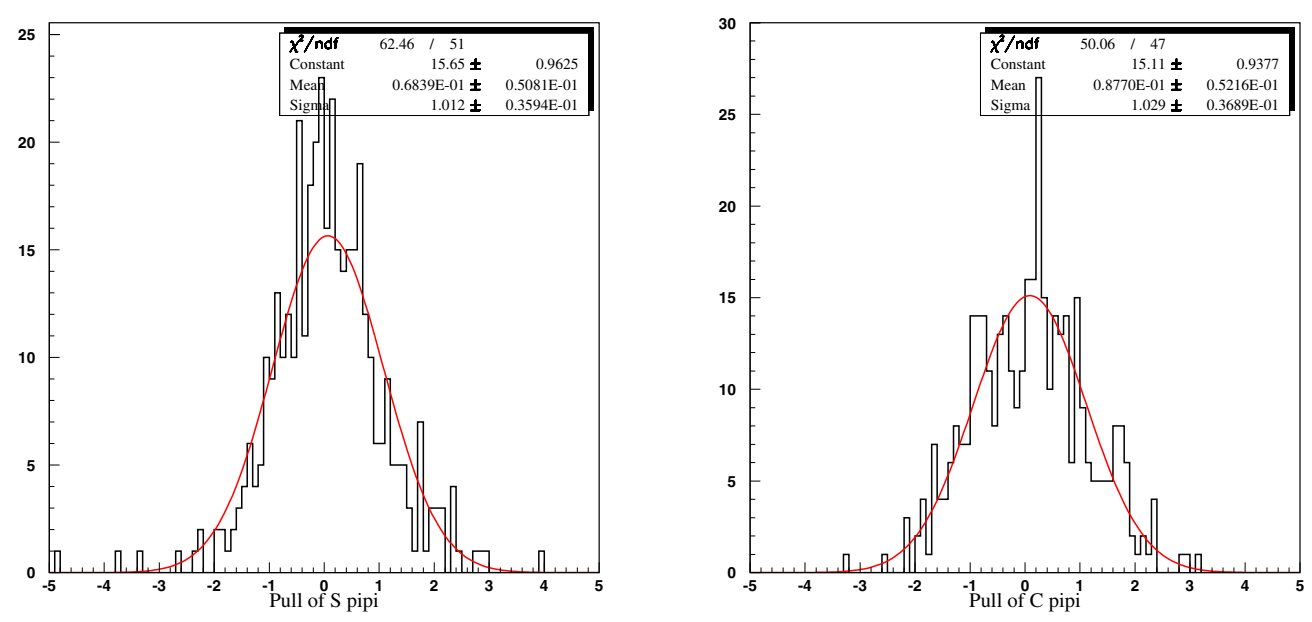

Figure 8.9: Pull distributions of $S_{\pi \pi}$ and $C_{\pi \pi}$ from fits to toy Monte Carlo experiments.

- Different signal and background Fisher discriminant distributions for each category.

- Different background $m_{\mathrm{ES}}$ distributions for each category.

For each experiment we choose random input values for $S_{\pi \pi}$ and $C_{\pi \pi}$ that satisfy $S_{\pi \pi}^{2}+C_{\pi \pi}^{2} \leq 1$, which reflects the requirement that the CP $\Delta t$ probability distributions (equation 8.1) are always positive and therefore physical. Finally, for simplicity, we do not include $B_{\text {Rec }}$ events in the toy Monte Carlo tests and only produce and fit $B^{0} \rightarrow h^{+} h^{\prime-}$ events.

Figure 8.9 displays the pull distributions of $S_{\pi \pi}$ and $C_{\pi \pi}$ from a sample of toy Monte Carlo experiments. These plots demonstrate that the fit produces no significant bias on the measured asymmetries and properly estimates the errors. Figure 8.10 shows the mean and width of these pulls versus the generated value of $S_{\pi \pi}$ and $C_{\pi \pi}$. We observe no indication of a dependence of the central value or error on the true value. In order to quantify our assessment of the bias, we examine the residual be- 

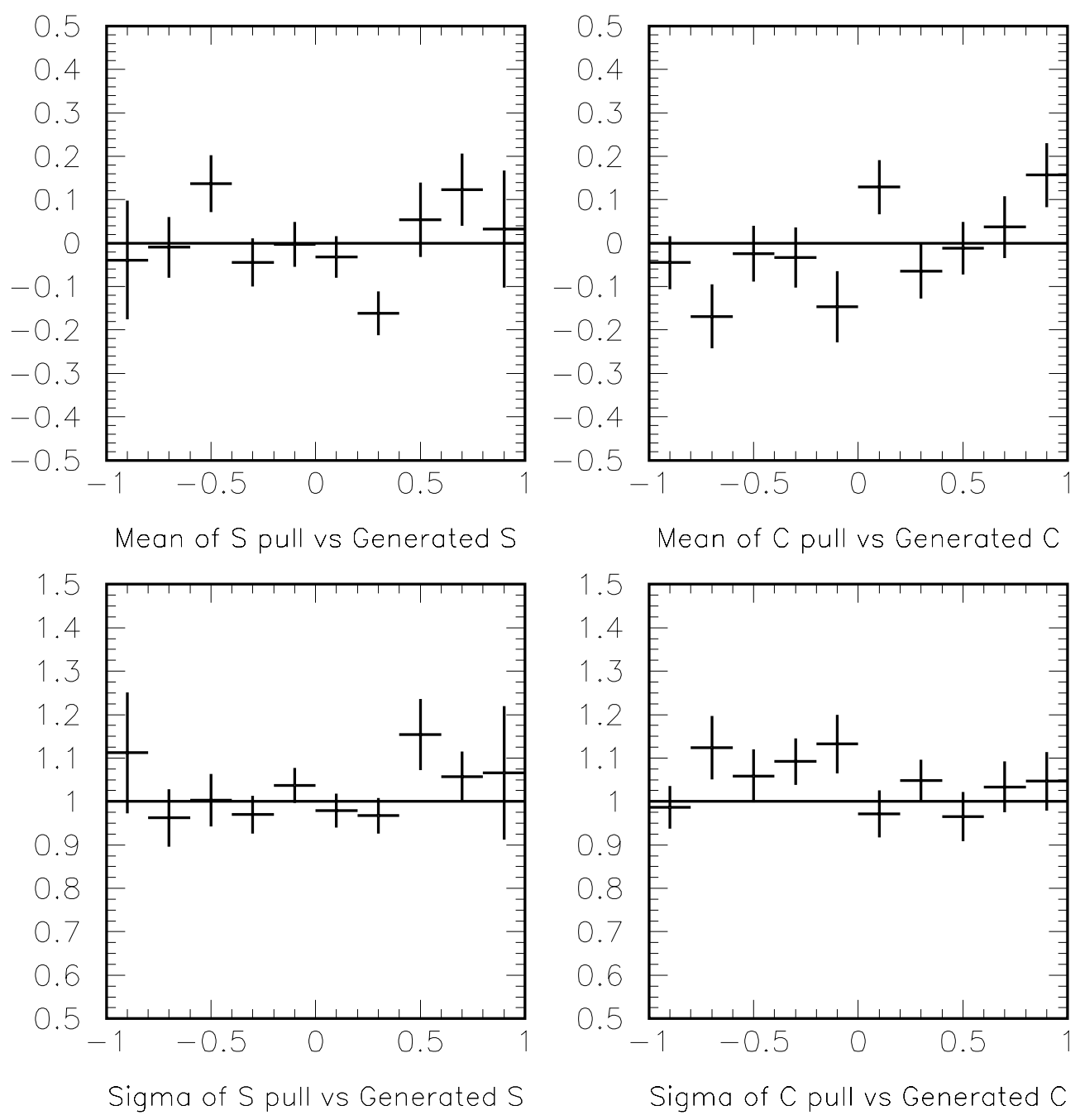

Figure 8.10: The mean and width of $S_{\pi \pi}$ and $C_{\pi \pi}$ as a function of their generated values from toy Monte Carlo experiments. 

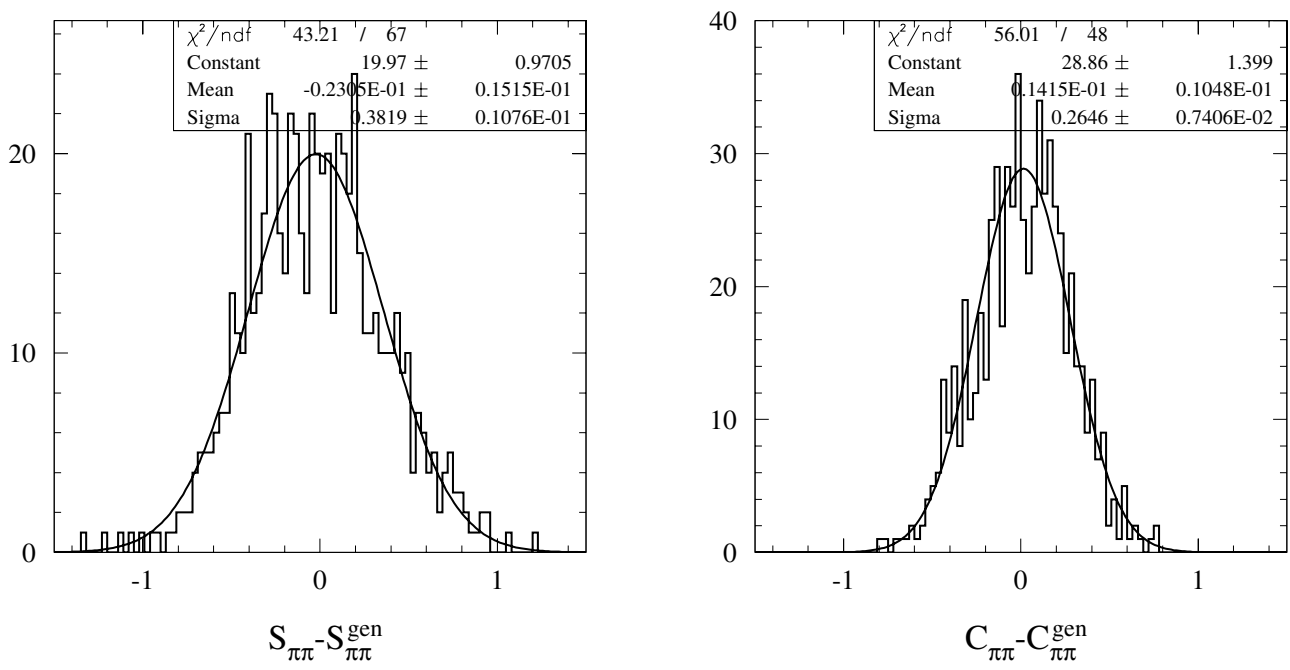

Figure 8.11: The distributions of residual (measured minus generated values) of $S_{\pi \pi}$ and $C_{\pi \pi}$ from fits to toy Monte Carlo experiments.

tween the measured and generated value of the asymmetries (see figure 8.11), and find $S_{\pi \pi}-S_{\pi \pi}^{\mathrm{Gen}}=-0.0231 \pm 0.01515$ and $C_{\pi \pi}-C_{\pi \pi}^{\mathrm{Gen}}=0.0142 \pm 0.0104$.

\subsubsection{Geant4 Monte Carlo}

Table 8.9 lists the results of test fits to large samples of simulated signal $B^{0} \rightarrow h^{+} h^{\prime-}$ events. The extracted values of $S_{\pi \pi}$ and $C_{\pi \pi}$ indicate no bias in the fit to pure sample of $B^{0} \rightarrow \pi^{+} \pi^{-}$decays or the fit with $B^{0} \rightarrow K^{+} \pi^{-}$events added in the appropriate ratio. Since the $B^{0} \rightarrow K^{+} \pi^{-}$decays are self tagging, they also permit measurement of the mixing frequency $\Delta m_{d}$. We measure both the $B$ lifetime, $\tau_{B}$, and mixing frequency, $\Delta m_{d}$, in the simulated $B^{0} \rightarrow K^{+} \pi^{-}$decays. The small bias which we observe is consistent with the expectation from the particular resolution function model used in this analysis [71]. 
Table 8.9: Summary of test fits on signal Monte Carlo samples of 50,000 $B^{0} \rightarrow \pi^{+} \pi^{-}$ and 200,000 $B^{0} \rightarrow K^{+} \pi^{-}$events with $S_{\pi \pi}=-0.40, C_{\pi \pi}=0, \tau_{B}=1.54 \mathrm{ps}$, and $\Delta m_{d}=0.472 / \mathrm{ps}$. The choice of number of events roughly approximates the ratio of the branching fractions.

\begin{tabular}{cccc}
\hline \hline & & $200 k K \pi$ & $200 k+$ \\
Parameter & $50 k B^{0} \rightarrow \pi^{+} \pi^{-}$ & $50 k B^{0} \rightarrow \pi^{+} \pi^{-}$ & $200 k B^{0} \rightarrow K^{+} \pi$ \\
\hline$S_{\pi \pi}$ & $-0.392 \pm 0.015$ & $-0.384 \pm 0.016$ & 0 (fixed) \\
$C_{\pi \pi}$ & $-0.004 \pm 0.010$ & $-0.001 \pm 0.011$ & 0 (fixed) \\
$\tau_{B}(\mathrm{ps})$ & 1.54 (fixed) & 1.54 (fixed) & $1.553 \pm 0.005$ \\
$\Delta m_{d}\left(\mathrm{ps}^{-1}\right)$ & 0.472 (fixed) & 0.472 (fixed) & $0.478 \pm 0.002$ \\
\hline \hline
\end{tabular}

\subsection{3 $B^{0} \rightarrow K^{+} \pi^{-}$Decays}

A measurement of $\tau_{B}$ and $\Delta m_{d}$ in the $B^{0} \rightarrow h^{+} h^{\prime-}$ sample tests the ML fit's sensitivity to the time and $\mathrm{CP}$ structure of $B$ decays which are embedded in a large background sample as well as the accuracy of the parameterizations of tagging and $\Delta t$. Such measurements are dominated by the signal $B^{0} \rightarrow K^{+} \pi^{-}$events which make up roughly $80 \%$ of the $B$ decays in the sample. In the $B^{0} \rightarrow h^{+} h^{-}$sample, we measure $\tau_{B}=1.56 \pm 0.07$ ps and $\Delta m_{d}=0.52 \pm 0.05 \mathrm{ps}^{-1}$, both consistent with the world averages $\tau_{B}=1.542 \pm 0.016 \mathrm{ps}$ and $\Delta m_{d}=0.489 \pm 0.008 \mathrm{ps}^{-1}$ [25]. In addition, by selecting samples enriched in $B^{0} \rightarrow K^{+} \pi^{-}$and $\bar{B}^{0} \rightarrow K^{-} \pi^{+}$, we plot $A_{\mathrm{K} \pi}^{\operatorname{mix}}(\Delta t)$ in figure 8.12 , and visually confirm that the data agrees with the parameterization used in the fit.

In section 8.1 we saw that for $B^{0} \rightarrow K^{+} \pi^{-}$decays, the time-dependent asymmetry between $B^{0}$ and $\bar{B}^{0}$ tagged decays exhibits the asymmetries $S_{K \pi}=0$ and 


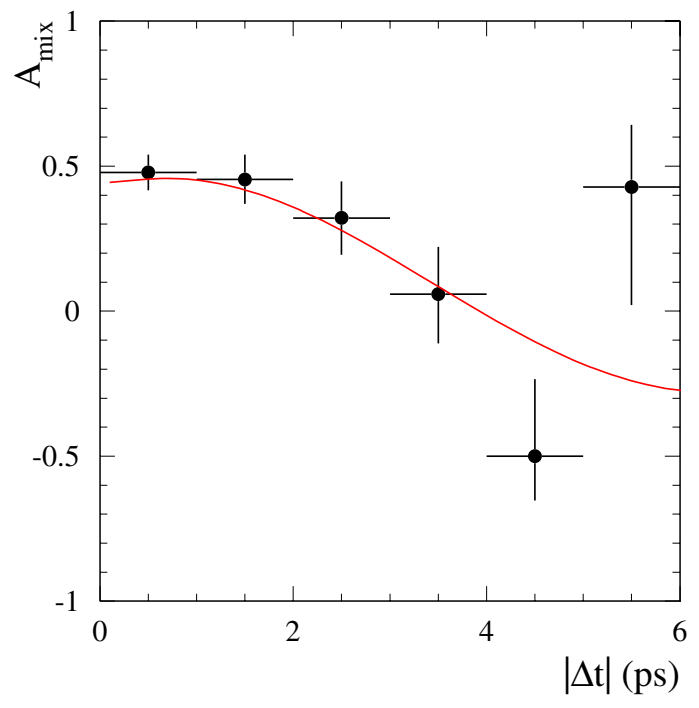

Figure 8.12: The time-dependent mixing asymmetry, $A_{\mathrm{K} \pi}^{\operatorname{mix}}(\Delta t)$, from samples enriched in $B^{0} \rightarrow K^{+} \pi^{-}$and $\bar{B}^{0} \rightarrow K^{-} \pi^{+}$decays. The curve represents the expectation including all signal and background decays, calculated from the PDFs used in the fit. 
$C_{K \pi}=-A_{K \pi}$ (see equation 8.4). As a test, we replace the mixing PDFs for the signal $B^{0} \rightarrow K^{+} \pi^{-}$species with CP PDF and measure these asymmetries. We find $S_{K \pi}=0.022934 \pm 0.15030$ and $C_{K \pi}=0.091753 \pm 0.11214$, which is consistent our measurement of $A_{K \pi}=-0.102 \pm 0.050 \pm 0.016$. Note that these two measurements of the $\mathrm{CP}$ asymmetries in this decay rely on different information. $C_{K \pi}$ is only sensitive to the flavor of the tagged $B$ meson and is blind to the flavor of the reconstructed mesons, which is the quantity the measurement of $A_{K \pi}$ depends on.

\subsection{Results}

The full ML fit to the combined $B^{0} \rightarrow h^{+} h^{\prime-}$ and $B_{\text {Rec }}$ samples finds $S_{\pi \pi}=0.024096 \pm$ 0.34036 and $C_{\pi \pi}=-0.30296 \pm 0.24796$, where the errors are statistical only. Figures 8.13a and 8.13b plot the $\Delta t$ distributions $B^{0}$ and $\bar{B}^{0}$ tagged events in a sample enriched in $B^{0} \rightarrow \pi^{+} \pi^{-}$decays. The selection of this sample follows the same procedure described in section 6.3.2. Figure $8.13 \mathrm{c}$ plots the asymmetry between the $B^{0}$ and $\bar{B}^{0}$ events, $A_{\pi \pi}^{\mathrm{CP}}(\Delta t)$. The curve on this plot represents the result of the fit to the full $B^{0} \rightarrow h^{+} h^{\prime-}$ fit, with the PDFs of each of the 8 species adjusted with the efficiency for appearing in the enriched sample.

\subsubsection{Alternative Measurements of $C_{\pi \pi}$}

Equation 8.2 illustrates that total number of $B^{0}$ and $\bar{B}^{0}$ tagged $B^{0} \rightarrow \pi^{+} \pi^{-}$events are related to $C_{\pi \pi}$, such that a large asymmetry between the $B^{0}$ and $\bar{B}^{0}$ yields are indicative of a large magnitude for $C_{\pi \pi}$. In the full $\mathrm{CP}$ fit, $C_{\pi \pi}$ is sensitive to both the $\Delta t$ PDF shape and integral, producing a result which is some average of the two. Therefore the fit has two handles on $C_{\pi \pi}$. This fact contributes to a more precise 


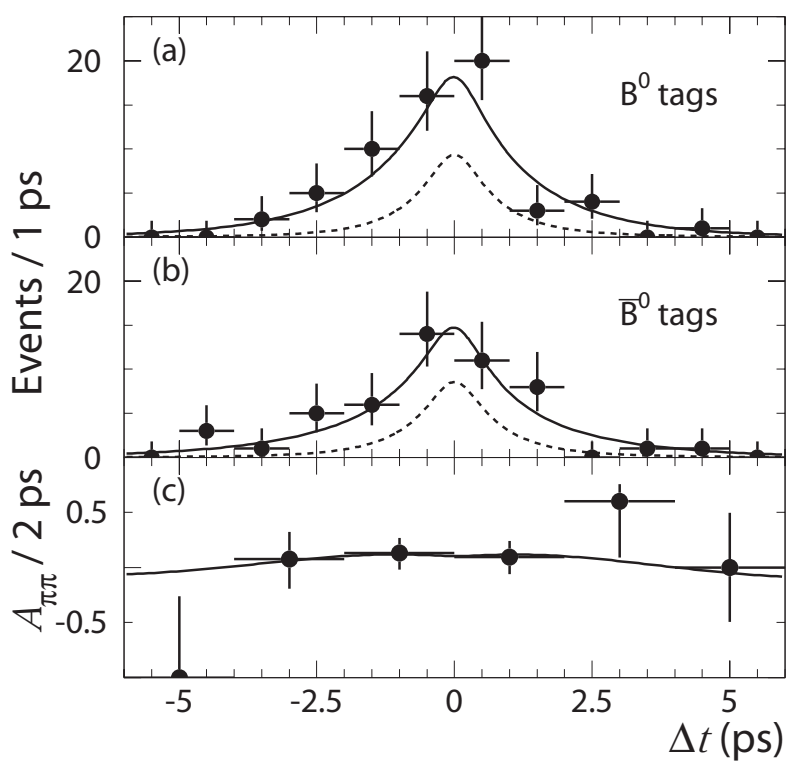

Figure 8.13: The $\Delta t$ distribution for (a) $B^{0}$ and (b) $\bar{B}^{0}$ tagged events and (c) the asymmetry $A_{\pi \pi}^{\mathrm{CP}}(\Delta t)$, from a sample enriched in $B^{0} \rightarrow \pi^{+} \pi^{-}$decays. The dashed curve represents the expected background contributions, primarily from continuum and $B^{0} \rightarrow K^{+} \pi^{-}$decays. The solid curve plots the signal and background contributions assuming the result from the fit to the full $B^{0} \rightarrow h^{+} h^{\prime-}$ sample. 
measurement of $C_{\pi \pi}$ than $S_{\pi \pi}^{1}$.

Using the branching fraction fit in chapter 6, we find $N_{\pi \pi}^{B^{0}}=47.070 \pm 9.8669$ and $N_{\pi \pi}^{\bar{B}^{0}}=47.838 \pm 9.5297$, suggesting $C_{\pi \pi} \approx 0$. In order to properly extract a timeintegrated measurement of $C_{\pi \pi}$, we remove the $\Delta t$ dependence of the likelihood by integrating all $\Delta t$ PDFs in analogy to equation (8.2). The resulting expressions are functions of $C_{\pi \pi}$, the tag, dilutions, tagging efficiencies and efficiency asymmetries. Using this likelihood, we fit $C_{\pi \pi}=-0.03792 \pm 0.30931$. As a cross-check, appendix B describes a measurement of $C_{\pi \pi}$ using time-integrated and $\Delta t$ shape separately.

\subsubsection{Fit without $\mathcal{F}$}

As discussed in section 8.3 we expect that the correlations of the Fisher discriminant with the $\Delta t$ error and tagging category produce no bias on the fitted values of the timedependent asymmetries. We test this by removing $\mathcal{F}$ from the ML fit and comparing the change in the result with expectation from toys. Without $\mathcal{F}$, the full fit measures $S_{\pi \pi}=-0.14576 \pm 0.35751$ and $C_{\pi \pi}=-0.26962 \pm 0.27320$, that is a change of -0.16 on $S_{\pi \pi}$ and +0.03 on $C_{\pi \pi}$ from the full fit. We find that $\approx 15 \%$ and $\approx 85 \%$ of toy Monte Carlo experiments exhibit a difference in $S_{\pi \pi}$ and $C_{\pi \pi}$, respectively, which is larger than what we observe in the $B^{0} \rightarrow h^{+} h^{\prime-}$ sample. Therefore we conclude that this correlation is unlikely to produce a bias in the results.

\subsubsection{Yields}

The time-dependent CP fit also provides a measurement of the yields of each species in the $B^{0} \rightarrow h^{+} h^{-}$sample. A comparison of this result (table 8.4) and the branching

\footnotetext{
${ }^{1}$ The fact that $B$ lifetime provides more $B$ decays at the maximum of the cosine rather than sine term in (8.1), also contributes to a smaller errors on the measurement of $C_{\pi \pi}$ than $S_{\pi \pi}$.
} 
fraction fit (table 6.9), reveals that the time-dependent fit finds $1.3 \%$ less signal $B^{0} \rightarrow \pi^{+} \pi^{-}$and $2.6 \%$ more signal $B^{0} \rightarrow K^{+} \pi^{-}$events. Since the fits rely on the same kinematic, event shape, and particle identification measurements, we would naively expect that the yields would be the same. However, as we discussed in the introduction to this chapter, $\Delta t$ and flavor tagging provide sufficient background discrimination to the time-dependent fit to significantly influence the yields. We fit toy Monte Carlo experiments with both the branching fraction and CP fits in order to evaluate the probability of obtaining the observed differences $\Delta N_{\pi \pi}^{S}=2$ and $\Delta N_{K \pi}^{S}=-15.5$ between the signal yields from the fits on the data. We find that $\approx 80 \%$ of experiments have $\left|\Delta N_{\pi \pi}^{S}\right|>2$ and $\approx 5 \%$ have $\left|\Delta N_{K \pi}^{S}\right|>15.5$.

\subsubsection{Goodness of Fit}

We assess the quality of the fit by comparing the errors and the likelihood from the data fit with expectations from toy Monte Carlo studies. Since the toy experiments only contain signal and background $B^{0} \rightarrow h^{+} h^{\prime-}$ events, we do not include the $B_{\text {Rec }}$ events in this comparison and fix the tagging and $\Delta t$ parameters of signal. Figure 8.14 plots the $S_{\pi \pi}$ and $C_{\pi \pi}$ errors and the $\chi^{2}=-2 \log L+C$ (see section 5.2.1) at the fit minimum. We find that $\approx 17 \%$ of the toy experiments have a smaller likelihood than the fit to data.

\subsection{Systematics}

Tables 8.10 and 8.11 summarize the systematic errors of the $C P$ asymmetries. We follow the same procedures as the branching fraction analysis (see section 6.4) to calculate the systematic errors which originate in $m_{\mathrm{ES}}, \Delta E, \mathcal{F}$, and $\theta_{c}$. For the time- 

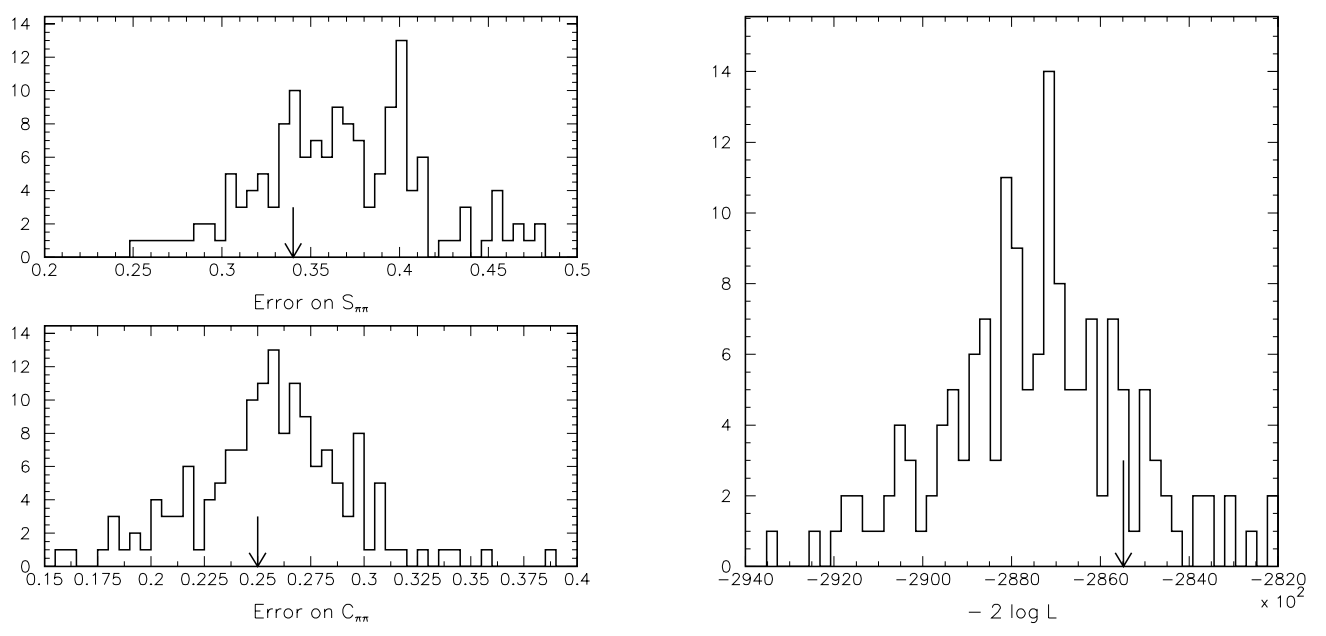

Figure 8.14: The error on $S_{\pi \pi}$ and $C_{\pi \pi}$ (left) and the $-2 \log$ likelihood from toy MC experiments (right). The arrows point to the values from the data fit.

dependent asymmetries we also consider additional potential sources of systematic errors:

- Toy MC bias - We apply any bias observed in the nominal toy Monte Carlo configuration as a systematic error (see section 8.5.1).

- SVT alignment - Since reconstruction of the $B$ decay vertices is dominated by the SVT's measurement of track parameters, $\Delta t$ is very sensitive to the knowledge of the relative positions of this detector's 340 silicon wafers. The translation/orientation parameters of each wafer, known as the SVT local alignment, are determined from $e^{+} e^{-} \rightarrow \mu^{+} \mu^{-}$and cosmic-ray muons events [73] and used in the reconstruction of charged tracks. We model potential systematic biases in the measurement of these parameters by considering possible deformations of the SVT with respect to a perfect alignment. We measure $S_{\pi \pi}$ and $C_{\pi \pi}$ 
in simulated $B^{0} \rightarrow \pi^{+} \pi^{-}$decays that have been reconstructed using three different misalignment scenarios, and assign the differences with no misalignment reconstruction as systematic errors.

- Boost $/ z$ scale - Knowledge of the $z$ scale (i.e. distances in the direction of the beams) and the boost directly impact the measurement of $\Delta t$. Studies of the interactions of beam electrons and positrons with the beam pipe material suggest an uncertainty on $z$ distance of $0.6 \%$ at the interaction region [72]. Meanwhile, errors in the measurement of the PEP-II beam energies translates to an uncertainty of $0.1 \%$ on the boost [38] $($ i.e. $\sigma \gamma \beta=(0.55) \cdot(0.001))$. In order to account for the influence of these possible effects, we refit the data sample with $\Delta t$ and $\sigma_{\Delta t}$ multiplied by 1.0066, and assign the differences with the nominal fit as systematic errors.

- $\Delta m_{d} / \tau_{B}$ - The nominal fit assumes the world average values of $\tau_{B}=1.542 \pm$ $0.016 \mathrm{ps}$ and $\Delta m_{d}=0.489 \pm 0.008 \mathrm{ps}^{-1}[25]$. In order to account for these uncertainties, we refit the data sample, varing $\Delta m_{d}$ and $\tau_{B}$ by their uncertainties.

- $\boldsymbol{B}_{\mathbf{R e c}} / \boldsymbol{K} \boldsymbol{\pi}$ - In order to assess any potential differences between the $B_{\text {Rec }}$ and $B^{0} \rightarrow h^{+} h^{\prime-}$ decays (see tables 8.1 and 8.2 ), we fit the recorded data twice: using signal $\Delta t$ resolution function and tagging parameters determined from either $B^{0} \rightarrow K^{+} \pi^{-}$or $B^{0} \rightarrow D^{(*)-} \pi^{+} / \rho^{+} / a_{1}^{+}$simulated events. We assign the difference in $S_{\pi \pi}$ and $C_{\pi \pi}$ measured using these two parameterizations as systematic errors.

The total additive systematic error is calculated as the sum in quadrature of the individual uncertainties. The final results are listed in table 8.12. 
Table 8.10: Detailed summary of systematic errors on yields and $C P$ asymmetries due to uncertainties in the PDF parameterizations, $\tau_{B}$, and $\Delta m_{d}$.

\begin{tabular}{ccccc}
\hline \hline Source & \multicolumn{2}{c}{$C_{\pi \pi}$} & \multicolumn{2}{c}{$S_{\pi \pi}$} \\
\hline$\mu_{\Delta E}$ & -0.0074 & +0.0141 & -0.0038 & +0.0080 \\
$\sigma_{\Delta E}$ & -0.0059 & +0.0041 & -0.0031 & +0.0023 \\
$\mu_{\mathcal{F}}$ & -0.0016 & +0.0011 & -0.0038 & +0.0038 \\
$\sigma_{\mathrm{L}}^{\mathcal{F}}$ & -0.0008 & +0.0004 & -0.0012 & +0.0011 \\
$\sigma_{\mathrm{R}}^{\mathcal{F}}$ & -0.0014 & +0.0010 & -0.0018 & +0.0018 \\
$\mu_{m_{\mathrm{ES}}}$ & -0.0186 & +0.0205 & -0.0048 & +0.0061 \\
$\sigma_{m_{\mathrm{ES}}}$ & -0.0020 & +0.0022 & -0.0056 & +0.0054 \\
$\theta_{c}^{K^{-}}$ & -0.0119 & +0.0066 & -0.0089 & +0.0007 \\
$\theta_{c}^{K^{+}}$ & -0.0057 & +0.0099 & -0.0008 & +0.0075 \\
$\theta_{c}^{\pi^{-}}$ & -0.0017 & +0.0071 & -0.0083 & +0.0006 \\
$\theta_{c}^{\pi^{+}}$ & -0.0070 & +0.0020 & -0.0011 & +0.0057 \\
$\Delta_{d}$ & -0.0104 & +0.0099 & -0.0009 & +0.0002 \\
$\tau_{B^{0}}$ & -0.0009 & +0.0006 & -0.0003 & +0.0002 \\
\hline \hline
\end{tabular}

Table 8.11: Global summary of systematic errors on $C P$ asymmetries.

\begin{tabular}{ccccc}
\hline \hline Source & \multicolumn{2}{c}{$C_{\pi \pi}$} & \multicolumn{2}{c}{$S_{\pi \pi}$} \\
\hline$m_{\mathrm{ES}}$ & -0.0187 & +0.0206 & -0.0074 & +0.0081 \\
$\Delta E$ & -0.0095 & +0.0147 & -0.0049 & +0.0083 \\
$\mathcal{F}$ & -0.0022 & +0.0015 & -0.0044 & +0.0043 \\
$\theta_{c}$ & -0.0151 & +0.0140 & -0.0122 & +0.0094 \\
Toy MC bias & 0.0000 & +0.0142 & -0.0231 & 0.0000 \\
SVT LA & -0.01 & +0.01 & -0.01 & +0.01 \\
boost $/ z$ scale & -0.003 & +0.003 & -0.001 & +0.001 \\
$\Delta m_{d}$ & -0.01 & +0.01 & -0.01 & +0.01 \\
$\tau_{B}$ & -0.0009 & +0.0006 & -0.0003 & +0.0002 \\
$B_{\text {Rec }} / K \pi$ & -0.0040 & +0.0040 & -0.0067 & +0.0067 \\
\hline Total & -0.0300 & +0.0356 & -0.0320 & +0.0221 \\
\hline \hline
\end{tabular}


Table 8.12: Central values and $90 \%$ C.L. intervals for $S_{\pi \pi}$ and $C_{\pi \pi}$ from the maximum likelihood fit. The errors are statistical and systematic, respectively.

\begin{tabular}{ccc}
\hline \hline Parameter & Central Value & $90 \%$ C.L. Interval \\
\hline$S_{\pi \pi}$ & $0.02 \pm 0.34 \pm 0.05$ & {$[-0.54,+0.58]$} \\
$C_{\pi \pi}$ & $-0.30 \pm 0.25 \pm 0.04$ & {$[-0.72,+0.12]$} \\
\hline \hline
\end{tabular}




\section{Chapter 9}

\section{Conclusions}

In this dissertation we detailed measurements of the branching fractions

$$
\begin{aligned}
\mathcal{B}\left(B^{0} \rightarrow \pi^{+} \pi^{-}\right) & =4.6 \pm 0.6 \pm 0.2, \\
\mathcal{B}\left(B^{0} \rightarrow K^{+} \pi^{-}\right) & =17.9 \pm 0.9 \pm 0.7, \text { and } \\
\mathcal{B}\left(B^{0} \rightarrow K^{+} K^{-}\right) & =<0.6(@ 90 \% \mathrm{CL}),
\end{aligned}
$$

the time-integrated CP asymmetry

$$
\mathcal{A}\left(B^{0} \rightarrow K^{+} \pi^{-}\right)=-0.102 \pm 0.050 \pm 0.016
$$

and the time-dependent CP asymmetries

$$
\begin{aligned}
& S_{\pi \pi}=0.02 \pm 0.34 \pm 0.03 \\
& C_{\pi \pi}=-0.30 \pm 0.25 \pm 0.03
\end{aligned}
$$

using BABAR's sample of 87.9 million $B \bar{B}$ decays. These results complement the current knowledge of all $B \rightarrow h h^{\prime}$ branching fractions and direct CP asymmetries, compiled in table 9.1. Recently, the Belle collaboration has measured $S_{\pi \pi}=-1.23 \pm$ $0.41_{-0.07}^{+0.08}$ and $C_{\pi \pi}=-0.77 \pm 0.27 \pm 0.08[84]$. 
As we discussed in chapter 2 , the $B^{0} \rightarrow h^{+} h^{--}$decays are potentially powerful probes of CKM matrix parameters. However, unlike the $\mathrm{CP}$ asymmetry in $B^{0} \rightarrow J / \psi K_{s}^{0}$ decays, hadronic uncertainties and not fully understood final state interactions prohibit a straight-forward interpretation of our measurements. Many strategies have been suggested for using these decays to extract the unitarity triangle angles $\alpha$ and $\gamma$ with different degrees of theoretical and experimental uncertainty [26-32]. Reference [74], by A. Höcker et al., systematically compares the application of some of these methods to our results using the CKMfitter [11] software. We will summerize the main conclusions of this document in this chapter. We will also briefly discuss one suggested method for interpreting the $B \rightarrow K \pi$ decays [31]. Note that the literature on this subject is extensive and our discussion does not aim to be comprehensive.

\subsection{The CKM matrix without $B^{0} \rightarrow h^{+} h^{\prime-}$ decays}

In section 2.2.4 we drew the unitarity triangle in the $\rho-\eta$ plane because these two Wolfenstein parameters are the most poorly known of the four which describe the CKM matrix. Figure 9.1 compiles several independent measurements of CKM parameters (listed in table 9.2), most notably $\epsilon_{k}, \Delta m_{s}, \Delta m_{d},\left|V_{u d}\right|,\left|V_{u s}\right|,\left|V_{u b}\right|,\left|V_{c d}\right|$, and $\sin 2 \beta$. The apparent agreement of these measurements, which is a non-trivial test of the consistency of the CKM model, permits the identification of an experimentally preferred area in the $\bar{\rho}-\bar{\eta}$ plane $^{1}$, where $\bar{\rho}=\rho\left(1-\lambda^{2} / 2\right)$ and $\bar{\eta}=\eta\left(1-\lambda^{2} / 2\right)$. Note that the $\left|V_{u b} / V_{c b}\right|$ constraint clearly prefers one of the solutions from the measurement of $\sin 2 \beta$.

\footnotetext{
${ }^{1}$ We will refer to this region as the "Standard CKM Fit" in the remainder of this chapter.
} 


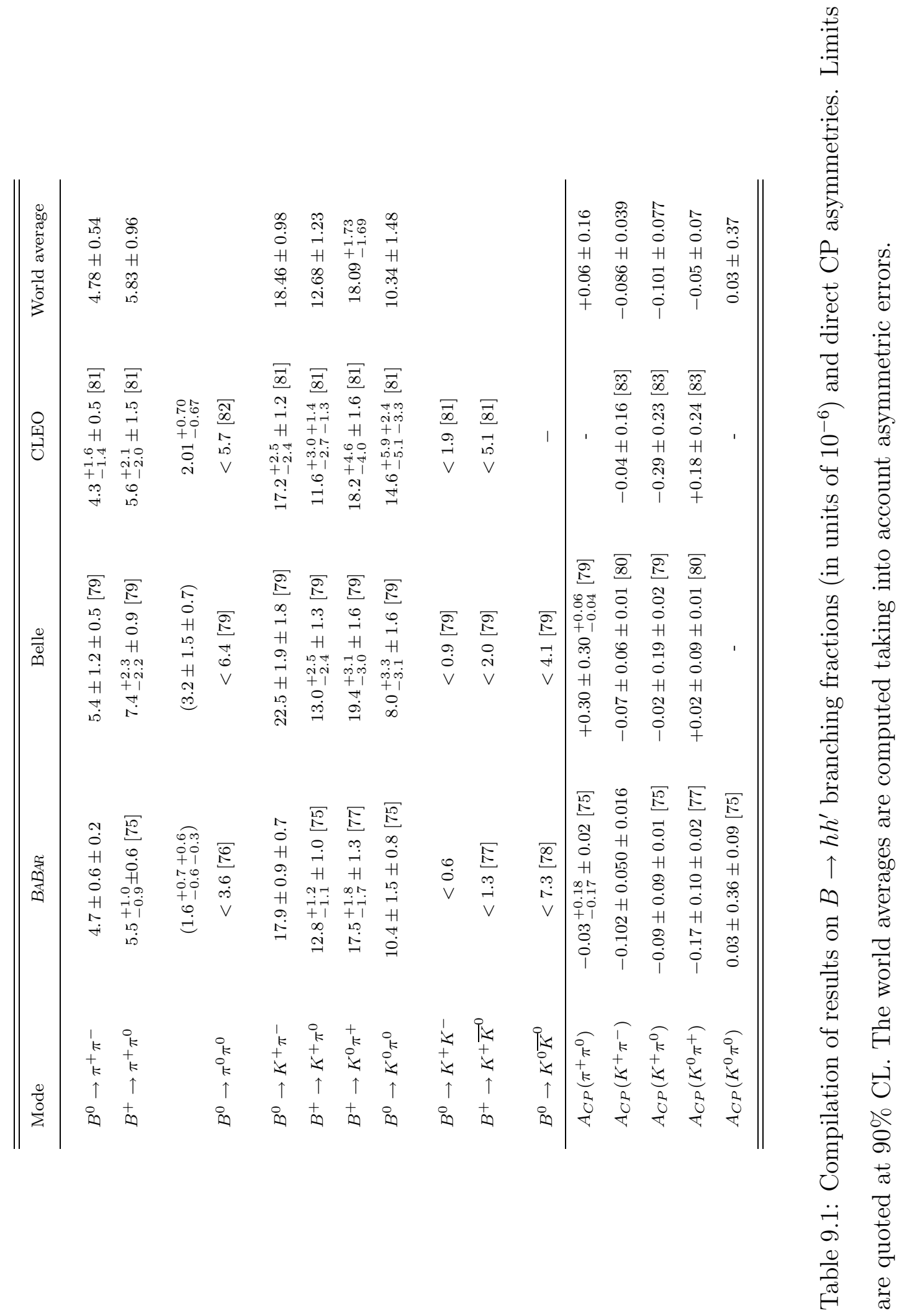




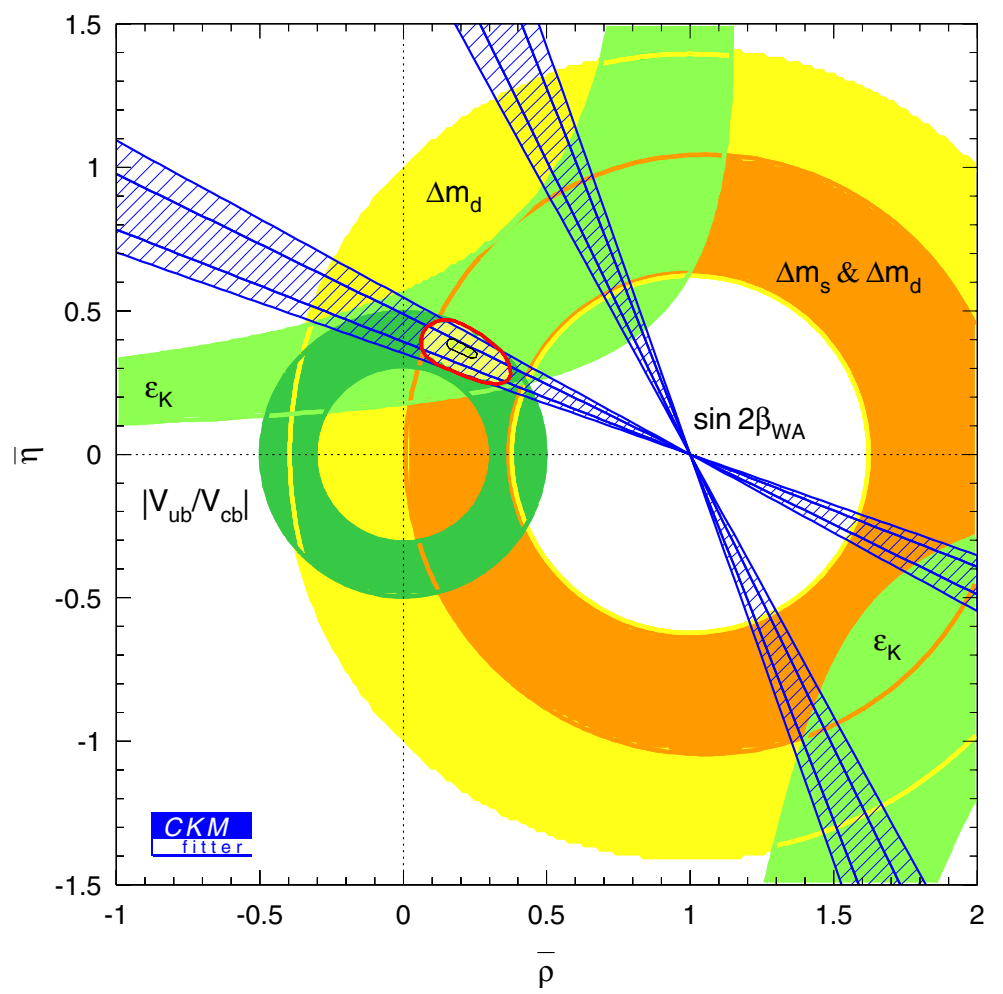

Figure 9.1: Confidence levels in the $\bar{\rho}-\bar{\eta}$ place based on inputs listed in table 9.2. The shaded areas indicate regions of $\geq 90 \%$ and $\geq 5 \%$ CLs, respectively. Also shown are the $\geq 5 \%$ CL domains of the individual constraints and the $\geq 32 \%$ and $\geq 5 \%$ CL constraints from the world average value of $\sin 2 \beta$. 


\begin{tabular}{|c|c|c|}
\hline Parameter & Value \pm Error $(\mathrm{s})$ & Source \\
\hline$\left|V_{u d}\right|$ & $0.97394 \pm 0.00089$ & neutron and nuclear $\beta$ decay \\
\hline$\left|V_{u s}\right|$ & $0.2200 \pm 0.0025$ & $K \rightarrow \pi \ell \nu$ decays \\
\hline$\left|V_{u b}\right|$ & $(4.08 \pm 0.61 \pm 0.47) \times 10^{-3}$ & LEP inclusive \\
\hline$\left|V_{u b}\right|$ & $(4.08 \pm 0.56 \pm 0.40) \times 10^{-3}$ & CLEO inclusive \& moments $b \rightarrow s \gamma$ \\
\hline$\left|V_{u b}\right|$ & $(3.25 \pm 0.29 \pm 0.55) \times 10^{-3}$ & CLEO exclusive \\
\hline$\left|V_{c d}\right|$ & $0.224 \pm 0.014$ & di-muon production: $\nu N$ (DIS) \\
\hline$\left|V_{c s}\right|$ & $0.969 \pm 0.058$ & $W \rightarrow X_{c} X(\mathrm{OPAL})$ \\
\hline$\left|V_{c b}\right|$ & $(40.4 \pm 1.3 \pm 0.9) \times 10^{-3}$ & excl./incl. \& CLEO moments analysis \\
\hline$\left|\epsilon_{K}\right|$ & $(2.271 \pm 0.017) \times 10^{-3}$ & PDG 2002 \\
\hline$\Delta m_{d}$ & $(0.496 \pm 0.007) \mathrm{ps}^{-1}$ & BABAR, Belle, CDF, LEP, SLD (2002) \\
\hline$\Delta m_{s}$ & Amplitude spectrum 2002 & \\
\hline $\sin 2 \beta_{\mathrm{WA}}$ & $0.734 \pm 0.055$ & BABAR, Belle, CDF \\
\hline$m_{c}$ & $(1.3 \pm 0.1) \mathrm{GeV}$ & PDG 2000 \\
\hline$m_{t}(\overline{\mathrm{MS}})$ & $(166.0 \pm 5.0) \mathrm{GeV}$ & CDF, D0 (PDG 2000) \\
\hline$m_{K}$ & $(493.677 \pm 0.016) \mathrm{MeV}$ & PDG 2002 \\
\hline$\Delta m_{K}$ & $(3.4885 \pm 0.0008) \times 10^{-15} \mathrm{GeV}$ & PDG 2002 \\
\hline$m_{B_{d}}$ & $(5.2794 \pm 0.0005) \mathrm{GeV}$ & PDG 2002 \\
\hline$m_{B_{s}}$ & $(5.3696 \pm 0.0024) \mathrm{GeV}$ & PDG 2002 \\
\hline$m_{W}$ & $(80.423 \pm 0.039) \mathrm{GeV}$ & PDG 2000 \\
\hline$G_{F}$ & $(1.16639 \pm 0.00001) \times 10^{-5} \mathrm{GeV}^{-2}$ & PDG 2002 \\
\hline$f_{K}$ & $(159.8 \pm 1.5) \mathrm{MeV}$ & PDG 2002 \\
\hline$B_{K}$ & $0.86 \pm 0.06 \pm 0.14$ & CERN CKM workshop 2002 \\
\hline \multirow[t]{2}{*}{$\bar{\eta}_{c c}$} & $1.46 \pm 0.41$ & Herrlich \& Nierste; \\
\hline & & update: CERN CKM workshop \\
\hline \multirow[t]{2}{*}{$\bar{\eta}_{c t}$} & $0.47 \pm 0.04$ & Herrlich \& Nierste; \\
\hline & & update: CERN CKM workshop \\
\hline \multirow[t]{2}{*}{$\bar{\eta}_{t t}$} & $0.573 \pm 0.007$ & Buras, Jamin, Weisz; \\
\hline & & update: CERN CKM workshop \\
\hline $\bar{\eta}_{B}(\overline{\mathrm{MS}})$ & $0.55 \pm 0.01$ & Buras et al. \\
\hline$f_{B_{d}} \sqrt{B_{d}}$ & $(230 \pm 28 \pm 28) \mathrm{MeV}$ & Lattice 2000 \\
\hline$\xi$ & $1.16 \pm 0.03 \pm 0.05$ & Lattice 2000 \\
\hline
\end{tabular}

Table 9.2: Inputs to the global CKM fit. If not stated otherwise: the first error includes statistics and experimental systematics and the second represent the systematic theoretical uncertainties. 


\subsection{Interpreting $S_{\pi \pi}$ and $C_{\pi \pi}$}

Reference [74] considers four hierarchical strategies for extracting CKM parameters from measurements of $S_{\pi \pi}$ and $C_{\pi \pi}$ :

1. Figure 9.2 - Assuming strong $S U(2)$ isospin symmetry with no electro-weak penguins and using the branching fractions for various $B \rightarrow \pi \pi$ final states, as described in section 2.5.1. Limited by the large $B^{0} \rightarrow \pi^{0} \pi^{0}$ upper limit, this method essentially provides no constraints in the $\bar{\rho}-\bar{\eta}$ plane and gives $|\theta| \equiv\left|\alpha-\alpha_{\mathrm{Eff}}\right|<51^{\circ}$ at $90 \% \mathrm{CL}$.

2. Figure 9.3 - Assuming $S U(3)$ flavor symmetry [34] and using (1) along with $\mathcal{B}\left(B^{0} \rightarrow K^{+} \pi^{-}\right)$, as described in section 2.5.2. As the figure illustrates, though this method provides a stronger limit than $(1)\left(|\theta|<29^{\circ}\right.$ at $90 \%$ CL), it still produces very weak constraints in the $\bar{\rho}-\bar{\eta}$ plane.

3. Figure 9.4 - Using (1) and assuming naive factorization (see section 2.5.3) to estimate $|R|=|P / T|$ (see equation 2.25) with no constraints on the relative strong phase $\delta[32]$. Here the penguin only $\mathcal{B}\left(B^{+} \rightarrow K^{0} \pi^{+}\right)$decay is used to estimate

$$
P=\sqrt{\frac{\tau_{B^{0}}}{\tau_{B^{+}}}} \frac{f_{\pi}}{f_{K}} \frac{1}{R_{\mathrm{th}}}\left|P_{K^{0} \pi^{+}}\right|,
$$

where $f_{\pi}=130.7 \pm 0.4 \mathrm{MeV}$ and $f_{K}=159.8 \pm 1.4 \mathrm{MeV}[85]$ are the pion and kaon decay constants, $R_{\text {th }}=0.98 \pm 0.05$ [31] is a theoretical estimate of $S U(3)$ breaking, and $\left|P_{K^{0} \pi^{+}}\right|^{2}\left|V_{t b}^{*} V_{t s}\right|=\left|P_{K^{0} \pi^{+}}\right|^{2}\left|V_{c b}^{*} V_{c s}\right|=\mathcal{B}\left(B^{0} \rightarrow K^{0} \pi^{+}\right)$. The tree amplitude, $T$, is deduced from $\mathcal{B}\left(B^{0} \rightarrow \pi^{+} \pi^{-}\right)$and the calculated value of $P$. This method constrains $|\theta|<20^{\circ}$ at $90 \%$ CL and excludes some $\bar{\rho}-\bar{\eta}$ regions, but it is limited by the lack of information on $\delta$, which prohibits exploiting 
the sign of $C_{\pi \pi}$. Note that no theoretical errors have been assigned for the dynamical assumptions.

4. Figure 9.5 - Using (1) and predictions of the $|R|$ and $\delta$ from QCD factorization [27] (see section 2.5.3). The constraints provided in the figure, which include the theoretical uncertainties quoted by the authors, are limited by the experimental uncertainties on $S_{\pi \pi}$ and $C_{\pi \pi}$. Negative $\bar{\eta}$ values are preferred due to the negative central value of $C_{\pi \pi}$ and the prediction of small $\delta$. Note that the fundamental limitation of this method is that the reliability of the calculation have not been fully established at this time [87]. For example, another calculation known as pQCD [86] predicts a large relative strong phase $\delta$.

Figure 9.6 presents a comparison of the predictions of the angle $\alpha$ from these methods, adding $\left|V_{u b}\right|$ to constrain the radius $\sqrt{\bar{\rho}^{2}+\bar{\eta}^{2}}$. Note that each successive strategy exchanges experimental limitations with theoretical uncertainties. Generally we find that estimates of $P / T$, which compensate for the lack of strong experimental limits (most notably on $\mathcal{B}\left(B^{0} \rightarrow \pi^{0} \pi^{0}\right)$ ), are necessary to produce a significant constraint in the $\bar{\rho}-\bar{\eta}$ plane. With the addition of information on $\delta$, strategy (4) permits comparison with limits from other measurements, discussed in the previous section and overlaid on each plot. Figure 9.5 illustrates that experimental accuracy is already competitive with the other CKM constraints and that consistency with the Standard Model is likely, assuming QCD factorization predictions are correct.

\subsection{Constraints from $B^{0} \rightarrow K^{+} \pi^{-}$}

Ratios of asymmetries and branching fractions of various $B \rightarrow K \pi$ decays have been recognized to provide non-trivial constraints on $\gamma$. For example reference [31], 


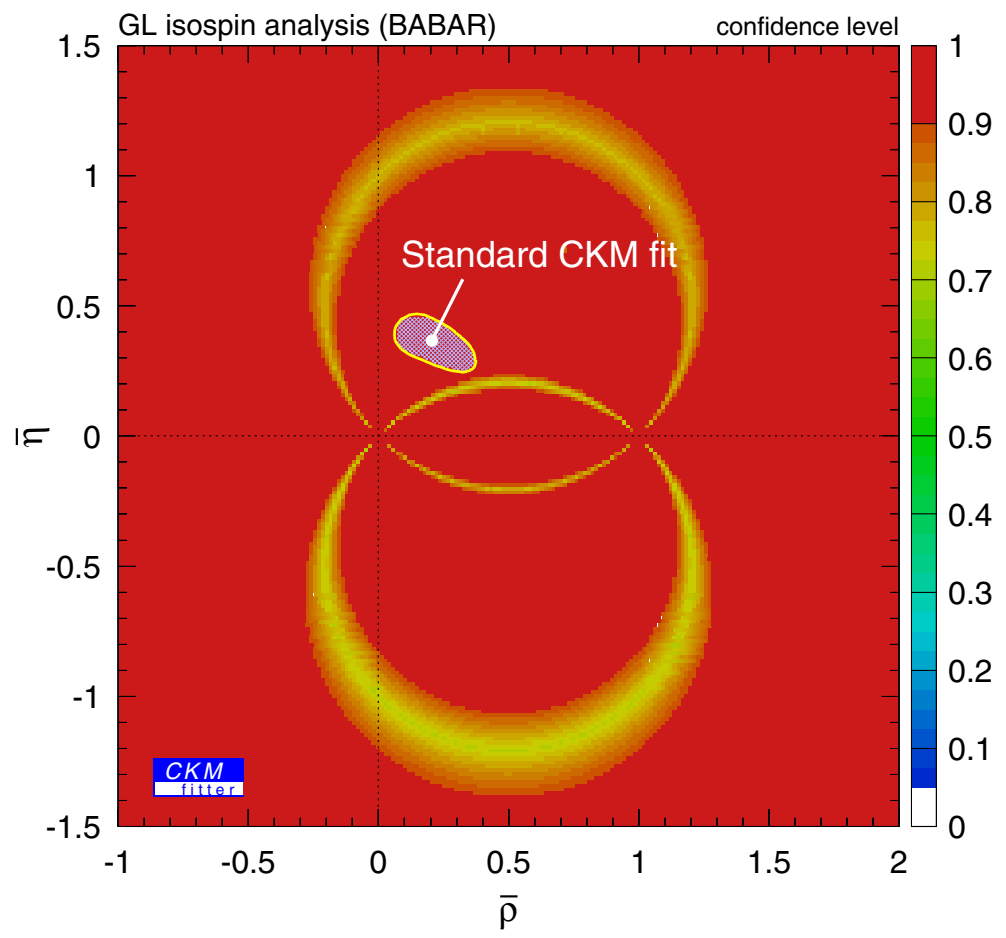

Figure 9.2: Confidence levels in the $\bar{\rho}-\bar{\eta}$ plane for strategy (1). Overlayed is the prediction from the SM fit. Refer to the text for a discussion. 


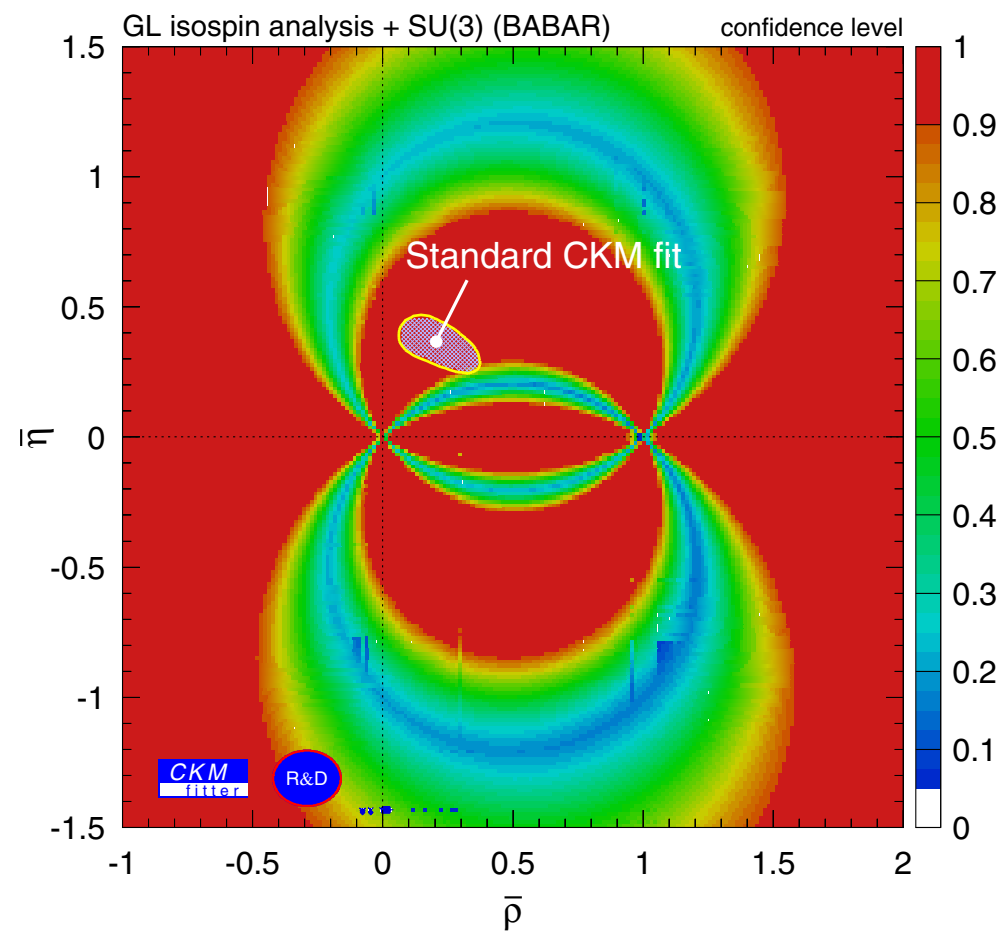

Figure 9.3: Confidence levels in the $\bar{\rho}-\bar{\eta}$ plane for strategy (2). Overlayed is the prediction from the SM fit. Refer to the text for a discussion. 


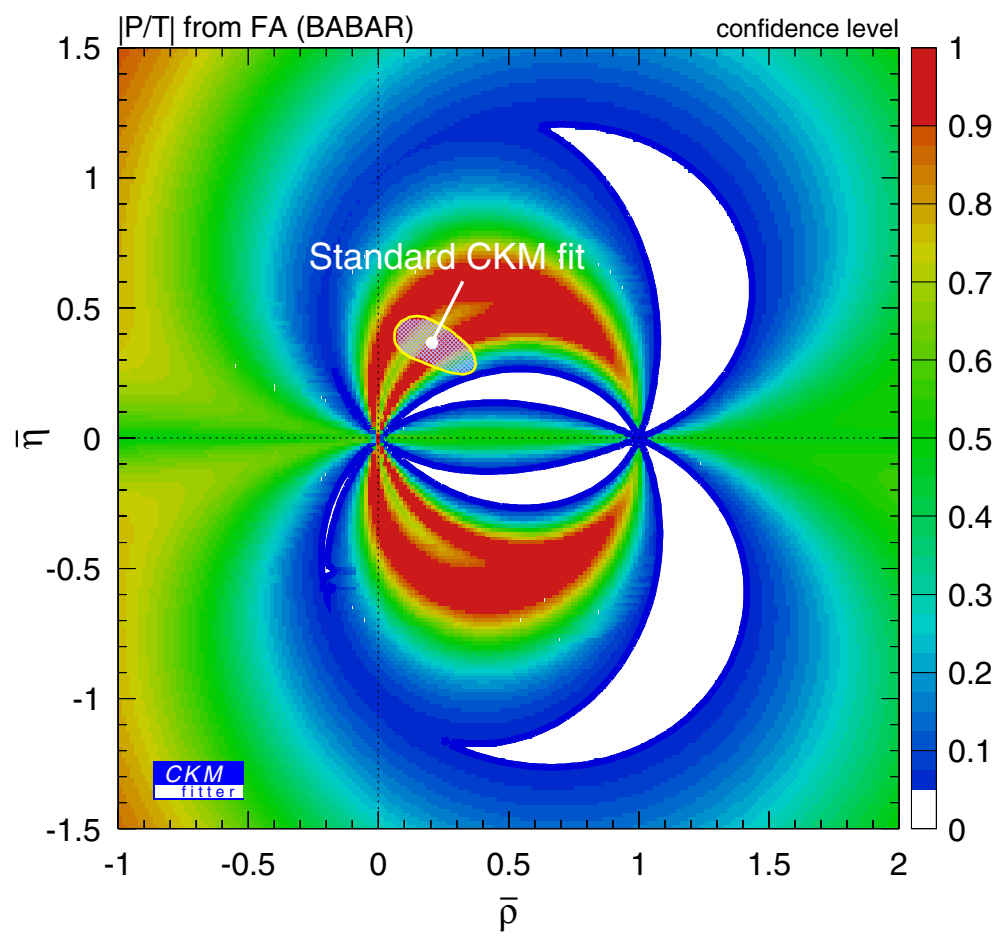

Figure 9.4: Confidence levels in the $\bar{\rho}-\bar{\eta}$ plane for strategy (3). Overlayed is the prediction from the SM fit. Refer to the text for a discussion. 


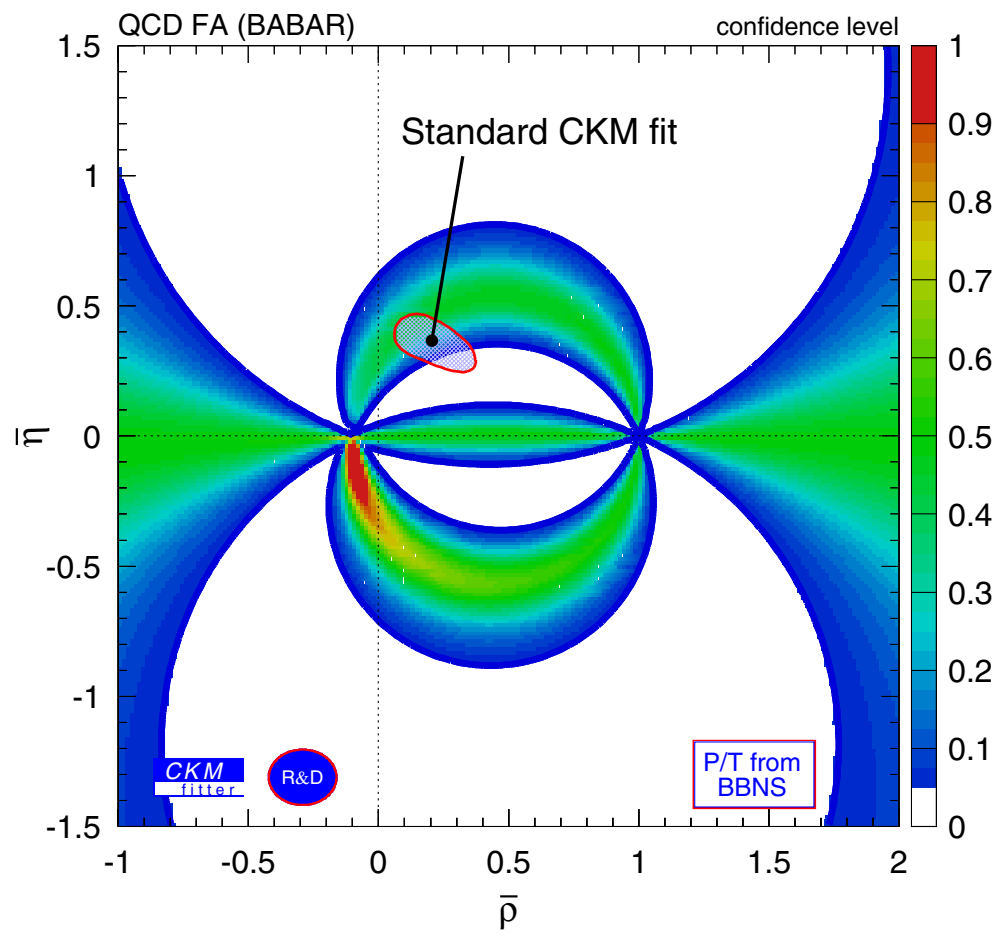

Figure 9.5: Confidence levels in the $\bar{\rho}-\bar{\eta}$ plane for strategy (4). Overlayed is the prediction from the SM fit. Refer to the text for a discussion. 


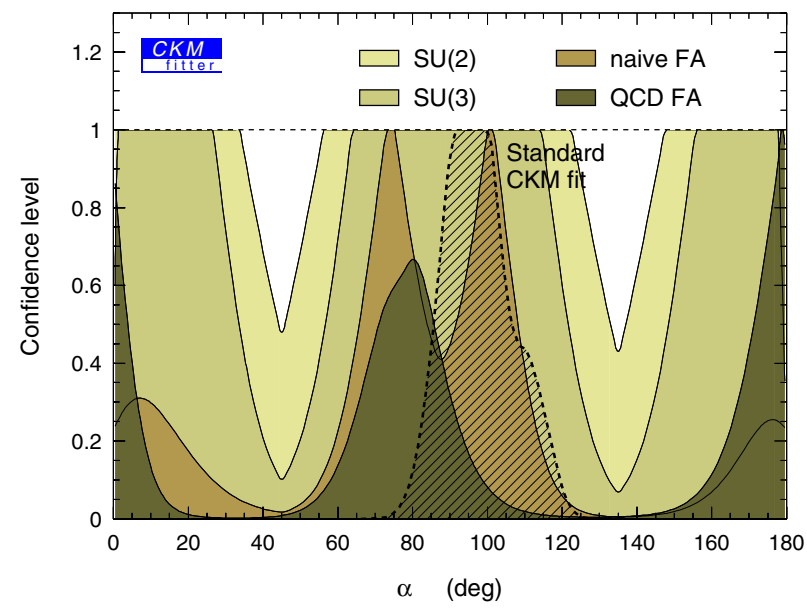

Figure 9.6: Confidence levels in $\alpha$ from strategies (1)-(4). The dashed-line/hashedarea represent the prediction from the SM fit.

assuming $S U(3)$ flavor symmetry and using factorization to estimate $S U(3)$ breaking effects, compares the quantities

$$
\begin{aligned}
& \left\{\begin{array}{c}
R \\
A_{0}
\end{array}\right\} \equiv\left[\frac{\mathcal{B}\left(B^{0} \rightarrow \pi^{-} K^{+}\right) \pm \mathcal{B}\left(\bar{B}^{0} \rightarrow \pi^{+} K^{-}\right)}{\mathcal{B}\left(B^{+} \rightarrow \pi^{+} K^{0}\right)+\mathcal{B}\left(B^{-} \rightarrow \pi^{-} \bar{K}^{0}\right)}\right] \frac{\tau_{B^{+}}}{\tau_{B^{0}}} \\
& \left\{\begin{array}{c}
R_{C} \\
A_{0}^{C}
\end{array}\right\} \equiv 2\left[\frac{\mathcal{B}\left(B^{+} \rightarrow \pi^{0} K^{+}\right) \pm \mathcal{B}\left(B^{-} \rightarrow \pi^{0} K^{-}\right)}{\mathcal{B}\left(B^{+} \rightarrow \pi^{+} K^{0}\right)+\mathcal{B}\left(B^{-} \rightarrow \pi^{-} \bar{K}^{0}\right)}\right], \text { and } \\
& \left\{\begin{array}{c}
R_{n} \\
A_{0}^{n}
\end{array}\right\} \equiv \frac{1}{2}\left[\frac{\mathcal{B}\left(B^{0} \rightarrow \pi^{-} K^{+}\right) \pm \mathcal{B}\left(\bar{B}^{0} \rightarrow \pi^{+} K^{-}\right)}{\mathcal{B}\left(B^{0} \rightarrow \pi^{0} K^{0}\right)+\mathcal{B}\left(\bar{B}^{0} \rightarrow \pi^{0} \bar{K}^{0}\right)}\right]
\end{aligned}
$$

with expectations, given different values of $\gamma$ and the relative penguin-tree strong phase in $B \rightarrow K \pi$. Though the statistical errors of the experimental results prohibit a strong conclusion, this analysis suggests that the data prefers large values of $\gamma$ than the global CKM in figure 9.1. 


\subsection{Prospects for $\alpha$}

As the $B$ factories continue to collect data and eventually upgrade in order to support even higher luminosities, we will obtain the two ingredients which are required for a measurement of the angle $\alpha$ with reduced sensitivity to theoretical uncertainties: the precise extraction of $S_{\pi \pi}$ and $C_{\pi \pi}$ and all of the $B \rightarrow \pi \pi$ branching fractions. Figure 9.7 displays projections of the statistical errors on $S_{\pi \pi}$ and $C_{\pi \pi}$ up to total recorded luminosity of $10000 \mathrm{fb}^{-1}$ at the $\Upsilon(4 s)$, where $\sigma_{S_{\pi \pi}}=0.03$ and $\sigma_{C_{\pi \pi}}=0.02$ is expected. In order to interpret such results without substantial input from modeldependent theoretical descriptions, we are likely to select the isospin analysis (see section 2.5.1) as the preferred strategy of extracting $\alpha$. Using this method, figure 9.8 displays the projected distribution of the residual $\theta=\alpha-\alpha_{\mathrm{Eff}}$, measured at different recorded luminosities. For this study, electroweak penguins were ignored, $\left|\lambda_{\pi^{0} \pi^{0}}\right|=1$, and the tagged $B^{0} \rightarrow \pi^{0} \pi^{0}$ and $\bar{B}^{0} \rightarrow \pi^{0} \pi^{0}$ branching fractions were assumed to be available with the central values of the world average in table 9.2. The figure indicates that an extraction of $\alpha$ with a clear solution for the value of $\theta$ may require $10000 \mathrm{fb}^{-1}$. However at lower integrated luminosities, the measurements in $B \rightarrow \pi \pi$ may test the $\mathrm{SM}$ if discrepancies with the expectations is large. 

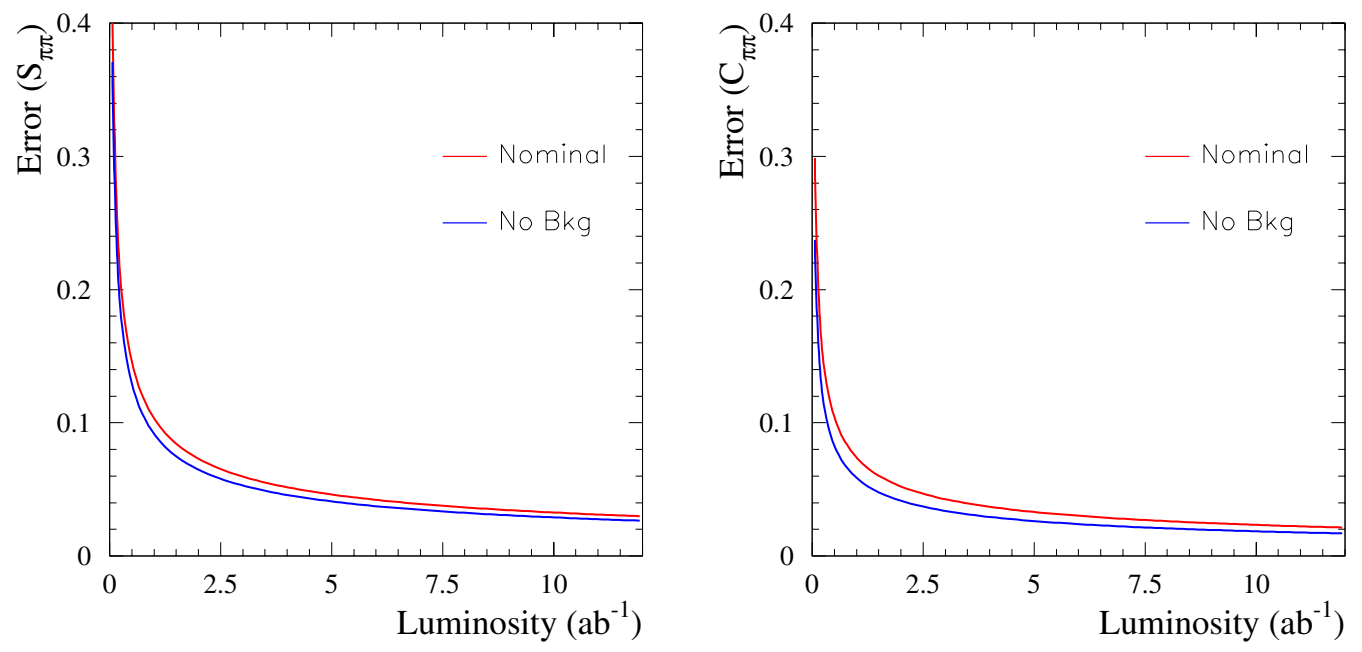

Figure 9.7: The statistical errors on (a) $S_{\pi \pi}$ and (b) $C_{\pi \pi}$ versus total recorded luminosity at the $\Upsilon(4 S)$.

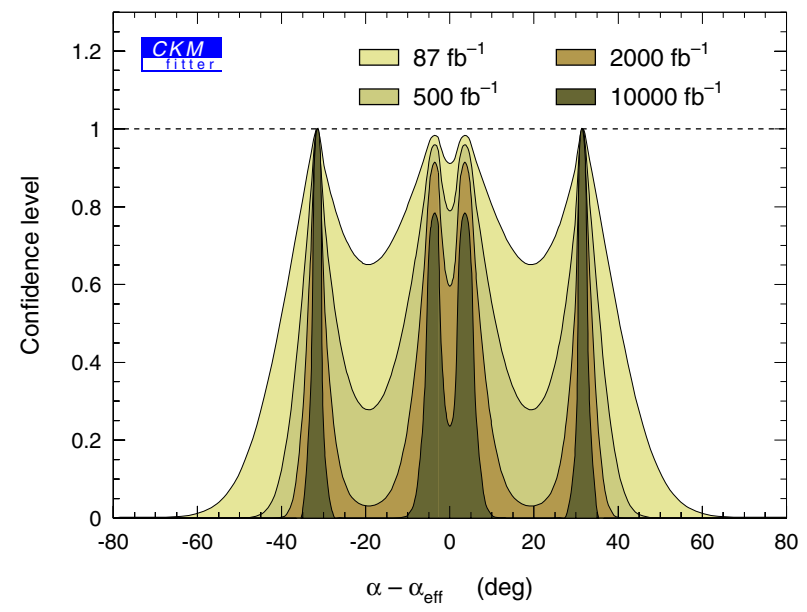

Figure 9.8: The residual $\theta=\alpha-\alpha_{\mathrm{Eff}}$ at different recorded luminosities, using full isospin analysis with no electroweak penguins and including tagged branching fractions for $B^{0} \rightarrow \pi^{0} \pi^{0}$. 


\section{Appendix A}

\section{Uncertainties on Time-dependent}

\section{Measurements}

Consider the approximate expression for the error on the measured time-dependent asymmetry $S_{\pi \pi}$ on a sample with $N_{\text {sig }}$ signal and $N_{\text {bkg }}$ background events:

$$
\sigma_{S_{\pi \pi}}=\frac{\Sigma\left(S_{\pi \pi}, \tau_{B} / \Delta m_{d}, \bar{\sigma}_{\Delta t}\right)}{\sqrt{N_{\mathrm{sig}}} \sqrt{\sum_{i=1} \bar{\epsilon}_{i}^{t} D_{i}^{2}}} \frac{\sqrt{1+N_{\mathrm{bkg}} / N_{\mathrm{sig}}}}{1+\left(A_{\mathrm{sig}} / A_{\mathrm{bkg}}\right)\left(N_{\mathrm{sig}} / N_{\mathrm{bkg}}\right)}
$$

where $A_{\text {sig }}$ and $A_{\mathrm{bkg}}$ are the amplitudes of $\mathrm{CP}$ asymmetry in the signal and background events, and $\Sigma\left(S_{\pi \pi}, \tau_{B} / \Delta m_{d}, \bar{\sigma}_{\Delta t}\right)$ and $Q_{i}=\bar{\epsilon}_{i}^{t} D_{i}^{2}$ quantify the influence of the $\Delta t$ resolution $\bar{\sigma}_{\Delta t}$ (see [19]) and the tagging performance, respectively. As expected this error is small for large values of the signal significance, $N_{\mathrm{sig}} / \sqrt{N_{\mathrm{sig}}+N_{\mathrm{bkg}}}$. Since the branching fraction ML fit discussed in chapter 5 maximizes this quantity, extending it to extract $S_{\pi \pi}$ also guarantees the smallest possible error $\sigma_{S_{\pi \pi}}$. However, if selections produce a sufficiently pure sample of $B^{0} \rightarrow \pi^{+} \pi^{-}$to permit ignoring backgrounds, statistical precision may be traded for gains in systematic uncertainties and simplicity. Unfortunately, the extremely small signal yield of any such selection on the available data-set renders extracting a measurement impractical. Therefore, since understanding the background events is unavoidable, it is preferable to include 
a significant number of background candidates in order to facilitate parameterizing them and accounting for systematic errors. The fit to the full $B^{0} \rightarrow h^{+} h^{--}$sample also provides this benefit. 


\section{Appendix B}

\section{Comment on Information on $C_{\pi \pi}$ from $\Delta t$}

\section{Shape}

The nominal ML fit described in chapter 8 is sensitive to both the $\Delta t$ shape and the asymmetry in the total number of $B^{0}$ and $\bar{B}^{0}$ events. Extracting a shape only measurement of $C_{\pi \pi}$ requires renormalization of the $B^{0} \rightarrow \pi^{+} \pi^{-} \Delta t \mathrm{PDF}$. We redefine

$$
\Gamma_{\bar{B}^{0}}^{B^{0}}(\Delta t)=\frac{\left(1+\frac{C_{\pi \pi}^{\text {Norm }}}{1+\left(\Delta m_{d} \tau\right)^{2}}\right)}{\left(1+\frac{C_{\pi \pi}^{\text {Shape }}}{1+\left(\Delta m_{d} \tau\right)^{2}}\right)} \frac{e^{-\frac{|\Delta t|}{\tau}}}{4 \tau}\left(1 \pm S_{\pi \pi} \sin \left(\Delta m_{d} \Delta t\right) \mp C_{\pi \pi}^{\text {Shape }} \cos \left(\Delta m_{d} \Delta t\right)\right),
$$

thereby decoupling the coefficient of the cosine term, $C^{\text {Shape }}$, from $C^{\text {Norm }}$, which contributes to the integral. Toy MC studies indicate that in a fit, both these quantities are unbiased estimators of $C_{\pi \pi}$. Using this PDF, we measure $S_{\pi \pi}=0.046468 \pm$ $0.28352, C_{\pi \pi}^{\text {Shape }}=-0.67683 \pm 0.25082$, and $C_{\pi \pi}^{\text {Norm }}=-0.0044425 \pm 0.30337$. Since tagging information from the same events are used to measure $C_{\pi \pi}^{\text {Shape }}$ and $C_{\pi \pi}^{\mathrm{Norm}}$, they are correlated and an average cannot be easily calculated for a direct crosscheck with the results of the standard ML fit with only one $C_{\pi \pi}$ parameter. However when toy Monte Carlo experiments are fit using both the $C_{\pi \pi}$ and the $C_{\pi \pi}^{\text {Shape }} / C_{\pi \pi}^{\text {Norm }}$ parameterizations, we find that differences of $C_{\pi \pi}^{\text {Shape }}-C_{\pi \pi}^{\text {Norm }}>0.67$ occur at $15 \%$ when $C_{\pi \pi}=-0.30$. 


\section{Bibliography}

[1] Lee, T.D. and Yang, C. N.(1956). Phys. Rev. 104, 254.

[2] Wu, C.S.,Ambler, E., Haywood, R. W., Hoppes, D. D., and Hudson, R. P. (1957). Phys. Rev. 105, 1413.

[3] Goldhaber, M., Grodzins, L., and Sunyar, A. W. (1958). Phys. Rev. 109, 1015.

[4] Sakharov, A. D. (197). JETP Letters 5, 24.

[5] J.H. Christenson, J.W. Cronin, V.L. Fitch, and R. Turlay, Phys. Rev. Lett. 13 (1964).

[6] N. Cabibbo, Phys. Rev. Lett. 10, 531 (1963); M. Kobayashi and T. Maskawa, Prog. Th. Phys. 49, 652 (1973).

[7] BABAR Collaboration, B. Aubert et al., Phys. Rev. Lett. 87, 091801 (2001). BELLE Collaboration, K. Abe et al., Phys. Rev. Lett. 87, 091802 (2001).

[8] Grimus, W. and Rebelo, M. N. (1997), Phys. Rev. B 427, 172.

[9] Branco, G. C., Lavoura, L., Silva, J. P., "CP Violation", Oxford Sicence Publications (1999). 
[10] [9] page 335 calculates this limit using Baluni, V. (1979). Phys. Rev. D 19, 2227, Crewther, R. J., Di Vecchia, P., Veneziano, G., and Witten, E. (1979), Phys. Rev. Lett. 88B, 123, and Altarev, I.S. et al. (1992), Phys. Rev. Lett. B276, 242.

[11] Hocker, A., Lacker, H., Laplace, S., Le Diberder, F. R., Eur. Phys. J. C21 (2001), 225-259.

[12] L. Wolfenstein, Phys. Rev. Lett. 51 (1983) 1945.

[13] L. L. Chau, W. Y. Keung, Phys. Rev. Lett. 53, (1984) 1802.

[14] V. F. Weisskopf, E. P. Wigner, Zeitschrift für Physik 63, 54. V. F. Weisskopf, E. P. Wigner, Zeitschrift für Physik 65, 18.

[15] R. Fleischer, hep-ph/0208083, to appear in the proceedings of BEAUTY2002.

[16] G.R. Farrar and M.E. Shaposhnikov, Phys. Rev. D 50 (1994); P. Huet and E. Sather, Phys. Rev. D 51, (1995); M.B. Gavela, M. Lozano, J. Orloff, and O. Pene, Nucl. Phys. B 430, (1994).

[17] Y. Nir, hep-ph/0109090, Lectures given at the 55th Scottish Universities Summer School in Physics.

[18] A.B. Carter and A.I. Sanda, Phys. Rev. D 45 (1980).

[19] P.F. Harrison and H.R. Quinn, The BABAR Physics Book.

[20] P.G. Harris et al,, Phys. Rev. Lett. 82 (1999).

[21] For a general review see Y. Nir and H. Quinn, Ann. Rev. Nucl. Part. Sci. 42, 211 (1992).

[22] BABAR Collaboration, B. Aubert et al., Phys. Rev. D 65, 051502 (2002). 
[23] Belle Collaboration, K. Abe et al., Phys. Rev. Lett. 89, 071801 (2002).

[24] For most the recent status of CKM parameter measurements and references see http://www.slac.stanford.edu/ laplace/ckmfitter/ckm_inputs2002.html.

[25] Particle Data Group, K. Hagiwara et al., Phys. Rev. D 66, 010001 (2002).

[26] M. Granau and D. London, Phys. Rev. Lett. 65 (1990) 3381.

[27] M. Beneke, G. Buchalla, M. Neubert, and C.T. Sachrajda, Nucl. Phys. B606, $245(2001)$

[28] M. Gronau, D. London, N. Sinha, and R. Sinha, Phys. Lett. B 514, 315 (2001); M. Gronau and J. Rosner, Phys. Rev. D 66, 053003 (2002).

[29] Y. Grossman and H.R. Quinn, Phys. Rev. D 58, 017504 (1998).

[30] J. Charles, Phys. Rev. D 59, 054007 (1999).

[31] R. Fleischer and J. Matias, Phys. Rev. D 66, 054009 (2002).

[32] M. Gronau and J. Rosner, Phys. Rev. D 65, 093012 (2002).

[33] The BABAR Collaboration, SLAC-PUB-9310, Contribution to ICHEP 2002.

[34] M. Neubert and J.L. Rosner, Phys. Lett. B441 (1998) 403; Phys. Rev. Lett. 81 (1998) 5076.

[35] Y. Grossmann and H.R. Quinn, Phys. Rev. D 58 (1998) 017504.

[36] M. Neubert, JHEP 0202 (2002) 028. 
[37] P. Oddone, Proceedings of the UCLA Workshop on Linear Collider B B-Factory Conceptual Design, ed. by D. Stork (1987).

[38] BABAR Collaboration, B. Aubert et al., Nucl. Instr. Methods A479, 1 (2002).

[39] Jacobson, B., Presentation at Computing in High Energy Physics (CHEP) 2000 Conference, Padova, Italy (2000), http://chep2000.pd.infn.it/abst/abs_a393.htm\}.

[40] http://www.objectivity.com. http://www.slac.stanford.edu/BFROOT/www/Public/Computing/Databases/index.shtml

[41] http://hep.ucsb.edu/people/lange/EvtGen.

[42] http://www.thep.lu.se/ torbjorn/Pythia.html.

[43] http://root.cern.ch.

[44] http://www.slac.stanford.edu/BFROOT/www/Computing/Offline/Kanga.

[45] http://wwwinfo.cern.ch/asdoc/hbook_html3/hboomain.html.

[46] P. Biloir, Nucl. Instr. Methods A225, 225 (1984).

[47] A. Drescher, et al., Nucl. Instr. Methods A237, 484 (1985).

[48] W.T. Ford, "Choice of Kinematic Variables in $B$ meson Reconstruction", BABAR Analysis Document 53 (2000).

[49] J.D. Bjorken, S. J. Brodsky, Phys. Rev. D 1, 413 (1970).

[50] S. Brandt, et al., Phys. Rev. Lett. 12, 57 (1964). E. Farhi, Phys. Rev. Lett. 39 1587 (1977). 
[51] G. C. Fox, S. Wolfram, Nucl. Phys. B149, 413 (1979).

[52] C. Caso, et al., Particle Data Group, Eur. Phys. J C3, 1 (1998).

[53] J. T. Boyd, "A Study of $B^{0} \rightarrow J / p s i \pi^{+} \pi^{-}$using the BABAR detector", Doctoral thesis submitted to the University of Manchester, Manchester, England (2001).

[54] http://ichep2000.hep.sci.osaka-u.ac.jp/.

[55] CLEO Collaboration, Y. Kubota, et al., Nucl. Instr. Methods A320, 66 (1992).

[56] CLEO Collaboration, D. Cronin-Hennessy et al., Phys. Rev. Lett. 85, 515 (2002);

[57] Belle Collaboration, K. Abe et al., KEK Report No. 2000-4.

[58] BABAR Collaboration, B. Aubert et al., Phys. Rev. Lett. 87 Lett. 87, 151802 (2001).

[59] http://www.lp01.infn.it/.

[60] BABAR Collaboration, B. Aubert, et al., Phys. Rev. D 65, 051502 (2002).

[61] http://moriond.in2p3.fr/EW/2002/.

[62] http://www.ichep02.nl/.

[63] BABAR Collaboration, B. Aubert, et al., Phys. Rev. Lett. 89, 281802 (2002).

[64] CLEO Collaboration, D. M. Asner, et al., Phys. Rev. D 53, 1039-1050 (1996).

[65] R. A. Fisher, Annals of Eugenics 7, 179 (1936). M. G. Kendall, A. Stuart, "The Advanced Theory of Statistics", Vol. III, 2nd Ed., Hafner Publishing, NY (1968). 
[66] J. Ocariz, M. Pivk, L. Roos, A. H ocker, H. Lacker, F. R. Le Diberder, "Background fighting in Charmless Two-body analyses", BABAR Analysis Document 346 (2002).

[67] ARGUS Collaboration, Z. Phys. C48, 543 (1990).

[68] A. Gritsan, http://www.slac.stanford.edu/〜gritsan/deltaE/study_de.ps.gz

G. Cavoto, http://babar-hn.slac.stanford.edu:5090/HyperNews/get/pubboard14/190.h

[69] M. Berzano, et al., BABAR Analysis Document 160 (2001).

[70] ARGUS Collaboration, Z. Phys. C62, 371 (1994).

[71] G. Raven, et al., BABAR Analysis Document 125 (2001).

[72] B. Dunwoodie, et al., "Radial and longitudindal length scale from beampipe structure", presented at Dec 2000 BABAR collaboration meeting.

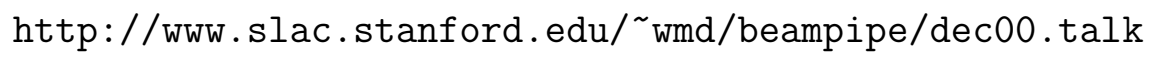

[73] D. Brown, et al., BABAR Analysis Document 486 (2002).

[74] A. H ocker, H. Lacker, M. Pivk, L. Roos, "Interperting CP Asymmetries in $B^{0} \rightarrow \pi^{+} \pi^{-}$Decays", BABAR Analysis Document 519 (2002).

[75] BABAR Collaboration (B. Aubert et al.), hep-ex/0207065.

[76] BABAR Collaboration (B. Aubert et al.), hep-ex/0207063.

[77] BABAR Collaboration (B. Aubert et al.), hep-ex/0206053. 
[78] BABAR Collaboration (B. Aubert et al.), hep-ex/0109005.

[79] Belle Collaboration (B.C.K. Casey et al.), hep-ex/0207090.

[80] K. Suzuki for the Belle Collaboration, Talk given at the the 31st International Conference on High Energy Physics (ICHEP'02), Amsterdam, The Netherlands $(2002)$

[81] CLEO Collaboration (D. Cronin-Hennessy et al.), hep-ex/0001010

[82] CLEO Collaboration (D.M. Asner et al.), Phys.Rev. D65 (2002) 031103

[83] CLEO Collaboration (S. Chen et al.), Phys.Rev.Lett.85:525-529,2000

[84] Belle Collaboration, K. Abe, et al., hep-ex/0301032.

[85] K. Hagiwara, et al., Phys. Rev. D 66, 010001 (2002).

[86] Y.-Y. Keum, H.-n. Li, Sanda, A. I., Phys. Rev. Lett. B504 6 (2001).

[87] Ciuchini, M. et al., Phys. Rev. Lett. B515 33 (2001). 